\title{
Supersymmetric Standard Model spectra from RCFT orientifolds
}

Een wetenschappelijke proeve op het gebied van

Natuurwetenschappen, Wiskunde en Informatica

\section{PROEFSCHRIFT}

ter verkrijging van de graad van doctor

aan de Radboud Universiteit Nijmegen

op gezag van de rector magnificus, prof. mr. S.C.J.J. Kortmann, volgens besluit van het College van Decanen

in het openbaar te verdedigen

op maandag 11 juni 2007,

om 15.30 uur precies.

DOOR

Tim Pieter Tjipko Dijkstra

Geboren op 13 januari 1978 te Hattem 
Promotor:

Prof. dr. A.N.J.J. Schellekens

Manuscriptcommissie: Prof. dr. R.H.P. Kleiss

Prof. dr. E.A. Bergshoeff, Rijksuniversiteit Groningen

Dr. L.R. Huiszoon, Barlaeus Gymnasium Amsterdam

ISBN: 978-90-6464-140-4

Copyright: (C)2007 Tim Dijkstra

Omslag: Charlotte Dijkstra

Druk: Ponsen \& Looijen B.V.

Het schrijven van dit proefschrift en het onderzoek waarop het gebaseerd is, is gedaan aan het Nationaal Instituut voor Kernen Hoge energie-Fysica (NIKHEF) te Amsterdam als onderdeel van programma String theory and quantum gravity van Stichting voor Fundamenteel Onderzoek der Materie (FOM) te Utrecht.

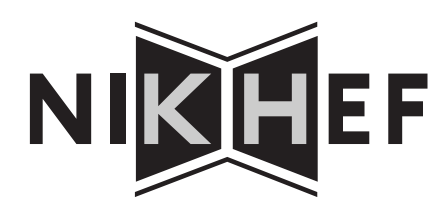


This thesis is based on the following articles:

Chapter 3 contains the text of:

T.P.T. Dijkstra, B. Gato-Rivera, F. Riccioni, A.N. Schellekens,

On orientifolds of $c=1$ orbifolds,

Nucl.Phys.B698:450-472,2004.

Chapter 4 contains the text of:

T.P.T. Dijkstra, L.R. Huiszoon, A.N. Schellekens,

Supersymmetric standard model spectra from RCFT orientifolds, Nucl.Phys.B710:3-57,2005.

Chapter 5 contains parts of (new work in sections 5.4.4, 5.4.5). :

P. Anastasopoulos, T.P.T. Dijkstra, E. Kiritsis, A.N. Schellekens, Orientifolds, hypercharge embeddings and the Standard Model.

Nucl.Phys.B759:83-146,2006. 

aan Anneke en Ton voor hun ondersteuning en stimulans 



\section{Contents}

1 Introduction 1

1.1 Why Quantum Gravity? . . . . . . . . . . . . . . . 1

1.2 Strings . . . . . . . . . . . . . . . . . . . . 2

1.3 Phenomenology . . . . . . . . . . . . . . . . . . . . 3

1.4 Realistic vacua . . . . . . . . . . . . . . . . . . . . . . . . . 4

1.5 The search for Standard model like vacua . . . . . . . . . . . . . . . 5

1.6 This thesis . . . . . . . . . . . . . . . . . . . 7

2 CFT and all that $\quad 9$

2.1 String theory . . . . . . . . . . . . . . . . . . . . . . . 9

2.2 Quantizing the String . . . . . . . . . . . . . . . . . . 11

2.3 Conformal Field Theory . . . . . . . . . . . . . . . . . . . . . 12

2.4 Simple currents . . . . . . . . . . . . . . . . . . . . . . . . . . . . . . . . . . . . . . . . . . . . .

2.5 Open strings . . . . . . . . . . . . . . . . . . . . . . . . . . . . 17

2.6 Additional Symmetries . . . . . . . . . . . . . . . . . . . . . . . . . . . 18

2.7 Algebraic constructions . . . . . . . . . . . . . . . . . . . . . . . . . . . . . . . . . . . . . . . . . . 20

2.8 Partition Functions . . . . . . . . . . . . . . . . . . . . . . . . . . . . 22

2.8 .1 Unoriented Strings . . . . . . . . . . . . . . . . . . . 24

2.8.2 Annulus \& Möbius strip . . . . . . . . . . . . . . . . 26

2.9 Open descendants . . . . . . . . . . . . . . . . . . . . . . . 26

2.10 Boundary States . . . . . . . . . . . . . . . . . . . . . . . . . . . . . . . . . . . . . . . . . . 27

2.11 Channel transformations . . . . . . . . . . . . . . . . . . . . . . . . . . . . . . . . . . . .

2.12 Ishibashi states . . . . . . . . . . . . . . . . . . . . . . 30

2.13 Transverse channel . . . . . . . . . . . . . . . . . . . . . . . 32

3 On Orientifolds of $\mathrm{c}=1$ Orbifolds 35

3.1 Introduction . . . . . . . . . . . . . . . . . . . . 35

3.2 Summary of circle results . . . . . . . . . . . . . . . . . . . . . . . . . . . . . . . . . . . . . . .

3.3 Orbifolds . . . . . . . . . . . . . . . . . . . . . . 40

3.3.1 Orientifolds for rational radius . . . . . . . . . . . . . . . . 41

3.3 .2 Orientifolds for arbitrary radius . . . . . . . . . . . . . . . 50

3.3.3 Orientifolds of exceptional MIPFs . . . . . . . . . . . . 53

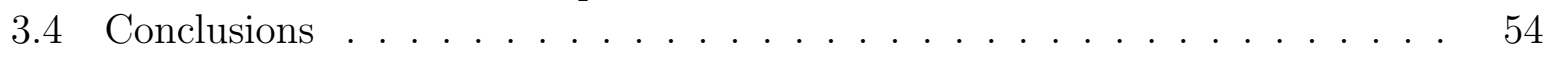


Appendix: Orbifold maps _. . . . . . . . . . . . . . . . . . . 56

4 Susy SM spectra from RCFT Orientifolds $\quad 59$

4.1 Introduction . . . . . . . . . . . . . . . . . . . . . 59

4.1.1 Brane configurations considered . . . . . . . . . . . . 60

4.1 .2 Chirality . . . . . . . . . . . . . . . . . 64

4.1.3 Scope of the search . . . . . . . . . . . . . . . . . 65

4.1 .4 Contents . . . . . . . . . . . . . . . . 67

4.2 Algebraic Model Building . . . . . . . . . . . . . . . . . . . . . . . . . . . . . . . . . .

4.2.1 Orientifold equivalences . . . . . . . . . . . . . . . 73

4.3 Massless Spectrum . . . . . . . . . . . . . . . . . . . . . . 74

4.3.1 The Oriented Closed String Spectrum . . . . . . . . . . . . . . 75

4.3.2 The Unoriented Closed String Spectrum . . . . . . . . . . . . . . 76

4.3.3 The Oriented Open String Spectrum _. . . . . . . . . . . . . 76

4.3.4 The Unoriented Open String Spectrum . . . . . . . . . . . . . . . . 77

4.4 Tadpoles \& Anomalies . . . . . . . . . . . . . . . . . . . . . . 78

4.4.1 Tadpole cancellation . . . . . . . . . . . . . . . . . . . . 78

4.4.2 Anomaly cancellation . . . . . . . . . . . . . . . . . . 79

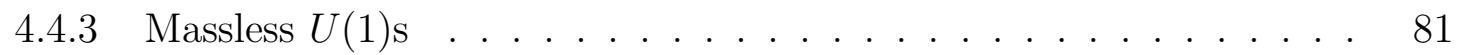

4.5 Results . . . . . . . . . . . . . . . . . . . . 82

4.5 .1 The numbers . . . . . . . . . . . . . . . 82

4.5 .2 Features of found spectra . . . . . . . . . . . . . . . . . . . . 84

4.5 .3 Higgs . . . . . . . . . . . . . . . . . . . . . 86

4.5.4 Hidden branes . . . . . . . . . . . . . . . . . . . . . . . 87

4.5.5 Gauge couplings . . . . . . . . . . . . . . . . . . 88

4.5.6 Varying number of chiral families . . . . . . . . . . . . . . . . . 91

4.6 The simplest case . . . . . . . . . . . . . . . . . . . . . . . . . . . . . . . . . . . . . . . . . . . . . .

4.7 Conclusions . . . . . . . . . . . . . . . . . . . . . . 95

Appendix: Boundary and Ishibashi orbits . . . . . . . . . . . . . . . 97

Appendix: Tables . . . . . . . . . . . . . . . . . . . . . . . . . . . 99

5 Generalizations $\quad \mathbf{1 1 5}$

5.1 Introduction . . . . . . . . . . . . . . . . . . . . 115

5.2 What we are looking for . . . . . . . . . . . . . . . . 120

5.3 Classification of bottom-up embeddings . . . . . . . . . . . . . . . . 124

5.3 .1 Orientable configurations . . . . . . . . . . . . . . . 125

5.3 .2 Charge Quantization . . . . . . . . . . . . . . . . 129

5.3 .3 Non-orientable configurations . . . . . . . . . . . . . . . . . . . 130

5.3.4 The cases $x=-\frac{1}{2}$ or $x=\frac{3}{2} \ldots \ldots \ldots \ldots \ldots \ldots$. . . . . . . . . . . . . . . . . . . 131

5.3.5 The case $x=1 \ldots \ldots \ldots \ldots$

5.3.6 Realizations with three brane stacks for $x=0 \ldots \ldots$. . . . . . 133

5.3.7 Realizations with three brane stacks for $x=\frac{1}{2} \ldots \ldots$. . . . . . . 134

5.3.8 Solutions with type E and F branes . . . . . . . . . . . . 135 
5.3.9 Solutions with type G branes . . . . . . . . . . . . . . . . 136

5.4 Top-down configurations and SM spectra . . . . . . . . . . . . . . . 137

5.4 .1 Scope of the top-down search . . . . . . . . . . . . . 137

5.4.2 Standard model brane configurations found . . . . . . . . . . . . 138

5.4.3 Bottom-up versus Top-down . . . . . . . . . . . . . . . . . . . . 142

5.4 .4 Intersection statistics . . . . . . . . . . . . . . . . . . . 148

5.4.5 Standard model brane configurations not found . . . . . . . . . . 152

5.4.6 Higgs, neutrino and mirror distributions . . . . . . . . . . . 155

Appendix: The unbiased search algorithm . . . . . . . . . . . . . . 157

$\begin{array}{ll}\text { Summary } & 171\end{array}$

$\begin{array}{ll}\text { Samenvatting } & 175\end{array}$

$\begin{array}{ll}\text { Dankwoord } & 179\end{array}$

$\begin{array}{lr}\text { Curriculum Vitae } & 180\end{array}$ 



\section{Chapter 1}

\section{Introduction}

\subsection{Why Quantum Gravity?}

The goal of theoretical physics has been and still is to capture the description of natural phenomena in mathematical laws. During the last centuries there has been tremendous progress on this enterprise. Since the days of Newton ever more phenomena can be carefully described and results of precision measurements can be successfully predicted. The motions of planets, the behaviour of gasses under changing pressure and temperature, chemical reactions, the outcome of collisions of protons, the hydrogen/helium ratio in the universe, and we could go on and on.

Again and again theories describing different phenomena appeared to be merely different viewpoints of the same object. The most famous examples is probably that of the theory of electricity and magnetism. These two phenomena for a long time seemed to be unrelated, until it was discovered that changing currents create magnetic fields and changing magnetic fields induce a current. The theories describing both fitted nicely into one beautiful unified framework. This process of unification has so far culminated in two monumental theories that describe, to great precision, all known physical processes and provide the basis of all natural sciences. These two theories, both created in the last century, are the Theory of General Relativity and Quantum Field Theory or more specifically the Standard Model.

The former describes what is Space $\&$ Time, and how Gravity is just its curvature. Its application is usually in the realm of big things, things like planets, galaxies and the universe as a whole. It is the successor of Newton's Laws on motion and gravity, which were degraded from laws to mere approximations. The predicted deviations from Newtons laws are in general small, but have been tested to great accuracy in for example the prediction of the motions of planets and the famous binary pulsar PSR1913+16. Totally new effects, not predicted or even possible in Newton's Laws are for example red shifting of light emanating from a massive object, the expansion of the universe and the slow down of fast moving clocks (actually time itself).

The second theory is a theory of the small, it encompasses the theory of elementary 
particles and the forces between them; the theory of atoms, light and radioactivity. The most extreme tests of this theory are done in accelerators such as at SLAC and Fermilab in the United States and CERN and DESY in Europe. At these places particles are collided at incredible energies, the products of these collisions are recorded at the highest accuracies.

All in all there has been no measurements that deviate from the predictions of these two theories with any significance. Yet this thesis is about a theory that has the ambition to go beyond these theories. There are several reasons why one would want to try this. To start with; the current situation is unsatisfactory from a philosophical point of view. Although in practice we can use one of the two theories to do predictions with great success, it is unsatisfactory we do not have one theory that is valid for all phenomena and situations. There are phenomena where a theory that describes gravity in a quantum-mechanical way is needed to fully understand the situation. Examples are the very early universe, black holes. Furthermore there is the hope that a unified theory of quantum gravity will give us insights in why certain natural constants have the values they have; Why are there three families of quarks and leptons? Why are there four large dimensions? Why is there a hierarchy of masses in the mass-spectrum of elementary particles? Why is the strength of gravity so low?

We clearly need a unified theory that is quantum mechanical and has both General Relativity and the Standard model as a limit.

\section{$1.2 \quad$ Strings}

One attempt, and the most successful to date, to find the theory of Quantum Gravity that describes nature is an endeavour that is usually called String theory. It is a wide and complicated subject in both mathematics and physics that contains theories and models that have sprung from a simple idea. The idea is that elementary particles are not pointlike in space-time, but rather one-dimensional objects: strings. This simple premise has far reaching consequences. The quantum theory that describes propagating strings necessarily contains as a limit a theory of a spin-2 field with the equations of motion equal to Einstein's Equations for the metric field. In other words it contains a quantum mechanical description of gravity. This big triumph has an immediate counter side, in that this can only be dealt with consistently in ten dimensions. This is a prediction which clearly contradicts experiment. But this drawback is not as bad as it seems, and will even turn out to be useful later on.

For a unified theory only gravity is of course not quite enough. We also need matter and the other forces. Now an important feature of string theory that distinguishes it from traditional gravity theories comes into play; String theory heavily constrains the types of matter one can couple to gravity. Matter particles are just like gravity some modes of the vibrating string. One is forced by several consistency conditions to carefully consider the possible configurations. At some point in time some string theorist have hoped that if one would study these conditions hard enough one would find the Standard model as the natural solution. During the last decade new insights has been developed, which has 
made this scenario become increasingly unlikely. Research described in this thesis gives additional support for that viewpoint. The majority of string theorists now recognize that although one is not free to choose the particle content of string theory, this still leaves a huge landscape of possibilities.

\subsection{Phenomenology}

As stated before, we have a theory of particle physics that works very well. Just like we can find Newton's theory of gravity back as a limit of General Relativity, we naturally want to find the Standard Model of particle physics back in string theory. Because the motivation of the research in this thesis was just that, finding back the standard model, we will summarize its key properties in this section, so we know what we are looking for.

Extensive research in the twentieth century has revealed that all particle experiments can basically be described by three forces acting on elementary particles. The most well known force is of course electromagnetism, which is described by a $U(1)$ gauge field mediated by a vector boson, the photon. Electrically charged particles, like the electron, feel this force. As far as we know there are two more forces, namely the strong and the weak force. These forces are also described by gauge theories similar to electromagnetism and have their own mediating particles: the weak vector bosons and the gluons. Together with electromagnetism they form the standard model gauge group:

$$
S U(3) \times S U(2) \times U(1)
$$

All matter we see round us is build up from a known ${ }^{1}$ set of fermionic particles bound together through these forces. These particles can be identified by the way they feel the forces; by their charges under the three forces and their mass. One important property of the Standard model is that it is chiral. Theoretically one can have matter particles with left and right handed chirality ${ }^{2}$, the standard model contains representations of only one handedness.

One final piece of the standard model is the Higgs particle, it is the only particle that has escaped detection so far. The hope is that this will be rectified in the near future in the new accelerator, the Large Hadron Collider, which is being build at the moment at CERN. The Higgs is a bit different from the other particles, it is a boson like the force particles, but it is not related to a gauge symmetry. By dynamical breaking of the $S U(2)$ gauge symmetry induced by the Higgs' vacuum expectation value, the Higgs is responsible for the fact that the matter particles have mass. Only after this breaking it is possible to identify familiar particles like the electron, which comes from coupling of the upper part of $L=\left(\begin{array}{l}\nu \\ e\end{array}\right)$ and $e^{c}$. Similarly $Q=\left(\begin{array}{l}u \\ d\end{array}\right)$ is coupled to weak singlet quarks $u^{c}$ and $d^{c}$. The photon is the linear combination of a $U(1)$ subgroup of the weak force and hyper charge (the

\footnotetext{
${ }^{1}$ Recent cosmological measurements seem to suggest that only $5 \%$ of the energy density of the universe is of the form we are familiar with. $25 \%$ has some unknown origin, but at least it seems to be some sort of matter. The last $70 \%$ is really different, it definitely can not be described by the standard model alone.

${ }^{2}$ Chirality is the direction a particle rotates around it is own axis with respect to the direction it moves.
} 
separate $U(1)$ in the Standard model gauge group) which stays massless after symmetry breaking.

\begin{tabular}{c|l|c|c|c|}
\multicolumn{2}{c|}{} & $S U(3)$ & $S U(2)$ & $U(1)$ \\
\hline \hline Spin 0 & $H$ & $\mathbf{1}$ & $\mathbf{2}$ & $-\frac{1}{2}$ \\
\hline \hline \multirow{3}{*}{ Spin $\frac{1}{2}$} & $Q$ & $\mathbf{3}$ & $\mathbf{2}$ & $+\frac{1}{6}$ \\
& $u^{c}$ & $\overline{\mathbf{3}}$ & $\mathbf{1}$ & $-\frac{2}{3}$ \\
& $d^{c}$ & $\overline{\mathbf{3}}$ & $\mathbf{1}$ & $+\frac{1}{3}$ \\
& $L$ & $\mathbf{1}$ & $\mathbf{2}$ & $-\frac{1}{2}$ \\
& $\nu^{c}$ & $\mathbf{1}$ & $\mathbf{1}$ & 0 \\
& $e^{c}$ & $\mathbf{1}$ & $\mathbf{1}$ & 1 \\
\hline \hline \multirow{3}{*}{ Spin 1 } & $Y^{\mu}$ & $\mathbf{1}$ & $\mathbf{1}$ & 0 \\
& $W^{\mu}$ & $\mathbf{1}$ & $\mathbf{3}$ & 0 \\
& $g^{\mu}$ & $\mathbf{8}$ & $\mathbf{1}$ & 0 \\
\cline { 2 - 5 }
\end{tabular}

Table 1.1: List of standard model representations

The last peculiar fact that is worth mentioning is that for all fermions in table 1.1 we have three copies (which are called families or generations), which differ only in their mass, or equivalently with what strength they couple to the Higgs.

\subsection{Realistic vacua}

Although it is clear as summarized in section 1.3 what particle content defines the standard model $^{3}$, we have to define better what precisely defines a standard model-like string theory vacuum. Of course at the very least one would want the fermions in table 1.1 to appear as chiral particles in the massless spectrum. Any other chiral states charged under the standard model gauge group would be unacceptable, we can however not exclude nonchiral pairs of states. String models are defined at scales much higher than is accessible by current experiments. Because mass terms for these pairs are not forbidden by a symmetry on the way down in energy there is always the possibility that such terms get generated. Of course this should be rigorously checked, but this is currently beyond what is technically possible.

In sections 4.1 .1 on page 60 and 4.1 .2 on page 64 we give a more thorough exposition on what we searched for, but to get an idea we will give a summary here.

First of all we search for models with space-time supersymmetry. There are of course phenomenological reasons to do so, but it also helps to restrict the search to theories

\footnotetext{
${ }^{3}$ Even the table is somewhat debatable. The weak-singlet neutrino $\left(\nu^{c}\right)$ is listed, although it is not strictly necessary. One can for example have Majorana mass terms to create neutrino masses without it. The models in chapter 4 all have three weak-singlet neutrinos, because of anomaly cancellation. In chapter 5 the number is left free.
} 
that are automatically free of certain pathologies, like tachyons. Naturally supersymmetry needs to get broken at some lower scale, however we treat that as a concern for later and do not derive any restrictions from that. The low energy spectrum thus contains in addition to the particles in table 1.1 on the preceding page their usual superpartners. There is one caveat and that is the superpartner of the Higgs. Because its superpartner is a fermion it will contribute to gauge anomalies. To be able to cancel those, the Higgs always comes in pairs in supersymmetric theories.

Then we have restrictions on the gauge group; It should at least contain the Standard model gauge group as a subgroup. It could be that the standard model gauge group is embedded in a bigger group like $S U(5)$ for example. In our initial paper we decided to concentrate on theories where the standard model gauge group is not embedded. Apart from embedding in a bigger group, it seems almost inevitable that the total gauge group contains additional factors (although we found vacua without), but this is not a big compromise. As long as there are no light states charged under both the standard model gauge group and the extra factors, these factors can be regarded as a hidden sector. Chiral states living entirely in the hidden sector could even be useful as dark matter.

Regarding the massless spectrum, we demand that the chiral spectrum of particles coupled to the standard model gauge group coincides with the chiral spectrum of table 1.1. In other words: no chiral exotics. The non-chiral spectrum we left free. Although these states do not get a mass at the order of the string scale, nothing prevents them from getting a mass by coupling to some moduli. Again these are calculations that should be done at some point, but this is not possible yet.

Of course if a model gets all the facts above right, it would already be a major achievement considering the fact it took the field so long to find an example in the context of orientifolds. However it is not quite what an experimental high energy physicist would call the standard model of particle physics. To be able to call it that, also the masses of the particles (couplings to the Higgs) and the strength of the gauge and other couplings should come out right. In general it should be possible to calculate these, but the required mathematical details are not worked out yet. There are also other complications, like for example the fact that we do not know how the Higgs sector is realized in nature

\subsection{The search for Standard model like vacua}

In this section we try to summarize work done on attempts to find phenomenologically realistic vacua prior to these thesis. This inevitably includes some technical terms that will not be introduced until further in this thesis.

Since 1984 there have been several attempts to search for standard model like string vacua. Two general classes may be distinguished: heterotic string constructions (either $(2,2)$ or $(0,2))$, with the standard model particles realized as closed strings, and type-I (orientifold, intersecting brane) constructions, characterized by open string realizations of the standard model. Other possibilities certainly exist (e.g. M-theory compactifications), and since new ways of obtaining the standard model are discovered about every five to ten 
years, it might be an illusion to think that we are close to a complete picture.

To explore the two classes mentioned above, we have essentially three methods at our disposal: free CFT constructions (free bosons and/or fermions and orbifolds thereof), geometric compactification (in particular Calabi-Yau compactifications) and interacting CFT constructions. All three have been applied successfully to heterotic string construction. The earliest example [87] of a three-family model was based on the Tian-Yau Calabi-Yau manifold with Euler number 6 and a non-trivial homotopy group $\pi_{1}$ (as far as we know still the only known manifold of this kind), used for Wilson line symmetry breaking. About a year later, the first orbifold examples were found [97, 17] (see also [42, 41, 68] for later developments). In [82] a three-family model was constructed using tensor products of $N=2$ minimal models, corresponding to a point in the moduli space of the Tian-Yau manifold. This model has an $E_{6}$ gauge group. By reducing the $(2,2)$ world-sheet symmetry to $(0,2)$ a large number of related models was constructed in [134] with $E_{6}$ or $S O(10)$ gauge symmetry. The symmetry in the bosonic sector can be reduced further to obtain many models with a gauge group $S U(3) \times S U(2) \times U(1) \times$ [Other factors] and three families of quarks and leptons [126]. Another class of three family models was obtained using free fermions in $[65,64,63]$, and extended in [45]. By means of a modified Calabi-Yau construction yielding $(0,2)$ spectra, several more three-family models were obtained in[101]. All the foregoing models lead to level 1 realizations of the standard model gauge groups $S U(3)$ and $S U(2)$, and hence their spectra contain fractionally charged states, or they have a non-standard normalization for the standard model $U(1)$ generator (or both) $[137,14,127]$. Unified models with standard model charge quantization can be constructed by using higher level Kac-Moody algebras, and indeed three family models were obtained [102]. Yet another approach to getting the standard model, based on strongly coupled heterotic strings, was presented in [61]. All of these heterotic three family models are supersymmetric. Constructing non-supersymmetric heterotic strings is much easier, but those theories are not stable, in general.

The open string road towards the standard model has been considerably more difficult. Until about 1995 this option was rarely taken seriously, with a few exceptions [124, 91]. This changed after the discovery of D-branes [117] and the observation in [138] that the problematic relation between the unification scale and the Planck scale could be avoided in open string theories. The construction of realistic theories is complicated by several new features, on top of those of the closed type-II theory to which the orientifold procedure is applied: boundary and cross-cap states and the requirement of tadpole cancellation. The first four-dimensional chiral, supersymmetric theory was constructed in [6], but it was still far from realistic. In order to get standard model-like spectra, in several later papers (e.g. $[30,3,98,32,16,28,105,106,44])$ the requirement of supersymmetry was relaxed, and only part of the tadpoles were cancelled, namely the RR tadpoles needed for consistency. The resulting theories are not stable, but otherwise consistent. The first semi-realistic supersymmetric theory that satisfies all tadpole conditions was presented in [49] and [50, 47]. However, these spectra contains chiral exotics, in addition to the standard model representations. In $[31,89]$ a three family supersymmetric Pati-Salam $(S U(4) \times S U(2) \times S U(2))$ model was constructed, but the two $S U(2)$ s do not emerge 
directly from string theory but from a diagonal subgroup of Chan-Paton groups using a "brane recombination" mechanism. In [46] supersymmetric Pati-Salam models were found that emerge directly from the CP-groups, but with additional chiral exotic matter, and in [48] supersymmetric $S U(5)$ GUT models with chiral exotics were presented. Most of the foregoing constructions are based on orientifolds of toroidal orbifolds [25, 83], except for [28] which uses the quintic Calabi-Yau manifold. In [43] standard model like spectra where obtained using branes at singularities. The first investigation of string spectra from open strings of orientifolds of Gepner models was done in [7], in 6 dimensions. The first analysis in four dimensions was done by [35]. These authors did not find chiral spectra, but got a first glimpse of the vast landscape of solutions in orientifolds. Further work on this kind of construction, including some chiral spectra, was presented in [35, 86, 5, 27, 38, 34, 2].

\subsection{This thesis}

The purpose of this thesis and the articles on which it is based is to find open string vacua that contain as the chiral spectrum just the particles of the Standard model. As is made clear by the previous section this has been a considerably tough objective and has been pursued by many people for a good portion of a decade before succeeding ${ }^{4}$. Finding examples required us to search through a very large number of brane configuration (in our searched we considered approximately $10^{19}$ ) This was only possible because we used algebraic methods to construct four dimensional open string vacua. Our search resulted in the first examples ${ }^{5}$ of supersymmetric standard model spectra, with the standard model group appearing directly as a Chan-Paton group and without chiral exotics. After completing our search we had compiled a set of 211,634 number standard model vacua. On this set we performed analysis of the features of the models we left free. We studied the distributions of various non-chiral states, some features of the total Chan-Paton gauge group and the values of the standard model gauge couplings at the string scale. To get a better understanding of how special a three family standard model is we expanded the search for a number of orientifolds to models with 1 to 9 families of chiral fermions. To make the first search computationally doable in finite amount of time we had to focus on a particular brane realization. We broadened this perspective in a latter search in a limited number of orientifolds to much more general constructions. Only few of the possible constructions do appear and when they appear they occur in greatly varying numbers. We analyse why this is the case and present a method to estimate the number of occurrences of a certain model in a set of vacua.

In chapter 2 we review some conformal field theory methods, especially the boundary state formalism, used in these constructions. In chapter 3 we use these methods on the simple but non-trivial example of $c=1$ orbifolds, were we can compare with a geometrical approach. We especially study different choices of orientifold projections and geometrical

\footnotetext{
${ }^{4}$ Shortly after we published our first paper, the first such example in the context of orientifolds of toroidal orbifolds was found[90].

${ }^{5}$ This was published in [59], a paper not included in this thesis.
} 
interpretations. The main part of the thesis is in chapter 4 . We first explain which brane constructions we considered and what exactly we regard as a supersymmetric standard model spectrum. Then we go on to explain how we build our models from orientifolds of simple current invariants. In section 4.3 on page 74 we show how one can determine the massless spectrum from the partition functions of these models. In the section that follows we introduce the consistency conditions we have to impose. In section 4.5 on page 82 we discuss the results of our first search. We present the number of spectra we found and for which models. We study the distributions of various features of the Chan-Paton gauge and the non-chiral spectrum. This chapter is concluded with an explicit example of one of the models and some concluding remarks. In chapter 5 we broaden the scope of our search to more general embeddings of the standard model gauge group and spectrum in the ChanPaton gauge group. For these models we only tried to solve all consistency conditions once per different standard model spectrum. To enumerate the possible embeddings we present a classification of the standard model hypercharge in the Chan-Paton gauge group. In this broadened search we now for example allow for models where anti-quarks are realized as antisymmetric tensors and find examples where this leads to supersymmetric $S U(5)$ spectra. This search results in 19345 chirally different spectra. Compared to the number of possible construction this is a small number. By statistical analyses of intersection matrices we try to understand why certain models occur more frequent then others or not at all. We find that the chiral intersection pattern of a model can be used in combination with the statistical data of the intersection matrix to estimate the frequency of the model in set of vacua we inspected. 


\section{Chapter 2}

\section{CFT and all that}

\subsection{String theory}

A string propagating through $D-1$ dimensional Lorentz invariant space, has a $D$-dimensional coordinate vector $\left(X^{\mu}, \mu \in 0 . . D-1\right)$, just like a point-particle. To parametrize the surface that is swept out by a string propagating one needs however two parameters, usually denoted $\tau$ (like proper time) and $\sigma$ (a coordinate along the string). See figure 2.1. In other words, we have $D$ coordinates which are functions of two parameters. From the perspective of the two-dimensional $\tau, \sigma$ plane we have $D$ scalar-fields living on a two dimensional surface (the world sheet), which happen to have some internal (Poincaré) symmetry. The simplest parametrization invariant action for the string is proportional to the area of the world-sheet. The naive expression one would write down for this contains a square root. Because this is difficult to work with one then introduces an auxiliary metric on the world sheet to simplify the formulation.

$$
S=-\frac{1}{4 \pi \alpha^{\prime}} \int d \sigma d \tau \sqrt{g} g^{a b} \partial_{a} X^{\mu} \partial_{b} X_{\mu}
$$

The energy-momentum tensor is found by an infinitesimal variation of the action with respect to the metric.

$$
T_{a b}=-\partial_{a} X \cdot \partial_{b} X+\frac{1}{2} g_{a b} \partial_{c} X \cdot \partial^{c} X
$$

Like in the previous equation we will suppress for the coming section the space time index from the equations and continue with only one $X$. While doing that we swept under the rug the fact that the field $X^{0}$ has 'the wrong sign' in the action. This is justified for our purpose, because when one takes in to account constraints coming from world sheet metric (for example by introducing ghosts) only $D-2$ 'normal sign' bosons survive as dynamic degrees of freedom. With that been said the world sheet metric will not be important either, it turns out that locally one can choose to work in the conformal gauge where this metric is absent:

$$
S=-\frac{1}{4 \pi \alpha^{\prime}} \int d \sigma d \tau\left\{\left(\partial_{\sigma} X\right)^{2}-\left(\partial_{\tau} X\right)^{2}\right\}
$$




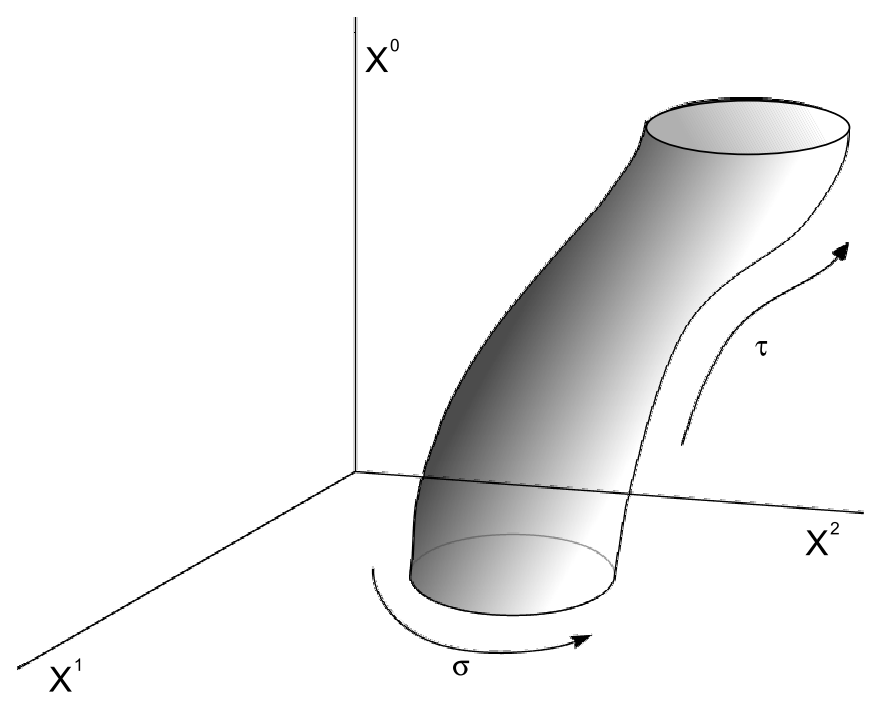

Figure 2.1: Closed string worldsheet

In the following I will put $\alpha^{\prime}=2$.

As said the string world sheet is parametrized by two real variables,

$$
\begin{aligned}
& \sigma \in \begin{cases}{[0,2 \pi)} & \text { closed } \\
{[0, \pi]} & \text { open }\end{cases} \\
& \tau \in(-\infty, \infty) \text {. }
\end{aligned}
$$

With this, we have not fully specified what we are talking about, we also have to impose some conditions on the fields $X$. First we assume our target space is compact:

$$
X=X+2 \pi R
$$

To get non-compact space one can take the limit where $R$ goes to infinity. Second, in the open string case, we have two possible boundary conditions:

$$
\begin{array}{lr}
\left.\partial_{\sigma} X\right|_{\sigma=0, \pi}=0 & \text { Neumann } \\
\left.\partial_{\tau} X\right|_{\sigma=0, \pi}=0 & \text { Dirichlet }
\end{array}
$$

Actually the Dirichlet case is the case where the end points are given $\left(\left.\delta X\right|_{\sigma=0, \pi}=0\right)$. They are fixed to something. Until we specify what they are fixed to, we can take without loss of generality the conditions as they are written down.

To be able to use to whole machinery of complex function theory we will first go to euclidean time $(\tau \rightarrow i \tau)$, and then map our cylinder/strip to the complex plane.

$$
z=e^{\tau+i \sigma}, \quad \bar{z}=e^{\tau-i \sigma}
$$


In these coordinates the action for our string (2.3) on the complex plane now takes the following form:

$$
S=\frac{1}{4 \pi} \int d^{2} z \partial X \bar{\partial} X
$$

From this equation we derive all the usual facts. The equation of motion $\partial \bar{\partial} X=0$ shows us that $\partial X$ is holomorphic and $\bar{\partial} X$ is anti-holomorphic. We can also rephrase the boundary conditions on open strings in terms of $z, \bar{z}$ :

$$
\partial X(z) \pm \bar{\partial} X(\bar{z})=0, \quad \text { for } z=\bar{z}\left\{\begin{array}{l}
\text { Neumann } \\
\text { Dirichlet }
\end{array}\right.
$$

As mentioned, the map (2.9) maps the cylinder to the plane, this is easy to see; the modulus of $z$ is given by $e^{\tau}$, so time flows radially with $\tau=-\infty$ mapped to the origin. For each time slice, space is swapped out by the phase of $z$, if $\sigma$ goes $0 \rightarrow 2 \pi, z$ makes a circle. The map for $\bar{z}$ is of course similar. For the open string things are a little bit different. Time is still radial, no change there, but open strings have a boundary, so we would expect to see one on the complex plane. In the map we have chosen the boundary arises naturally at the real axis. If $\sigma$ goes $0 \rightarrow \pi$ this sweeps out the upper half-plane for $z$. The left side of the axis is the boundary at $\sigma=\pi$ from $\tau=-\infty \rightarrow+\infty$ and the right side the boundary at $\sigma=0$. The phase of $\bar{z}$ is minus that of $z$, so in the $\bar{z}$-plane space sweeps out the lower half-plane, this has the effect that functions of $z$ are defined for $\operatorname{Im} z>0$ and functions of $\bar{z}$ only for $\operatorname{Im} \bar{z}<0$. We will see why this is important in a minute.

\subsection{Quantizing the String}

The equations of motion coming from action (2.10) imply that we can expand $X$ as follows:

$$
X=x-i a_{0} \ln z+i \sum_{n} \frac{1}{n} a_{n} z^{-n}+\bar{x}-i \bar{a}_{0} \ln \bar{z}+i \sum_{n} \frac{1}{n} \bar{a}_{n} \bar{z}^{-n} .
$$

In this equation $x+\bar{x}$ is obviously the center of mass coördinate. After imposing canonical commutation relations between $X$ and $\frac{i}{4 \pi} \partial_{\tau} X$ it becomes clear that the zero modes have to satisfy:

$$
\left[x+\bar{x}, \frac{a_{0}+\bar{a}_{0}}{2}\right]=i,
$$

so we can safely identify $\frac{a_{0}+\bar{a}_{0}}{2}$ with center of mass momentum. For a non-compact target space, periodicity of $X$ imposes $a_{0}-\bar{a}_{0}=0$, but because we live in a compact space (2.6) the restriction becomes:

$$
a_{0}-\bar{a}_{0}=R l, \quad l \in \mathbb{N}
$$

In other words, we can define a winding number operator $w=\frac{1}{R}\left(a_{0}-\bar{a}_{0}\right)$, that measures the number of times we go around target space if we go around the string. Furthermore 
single-valuedness of vertex operators $e^{i k X}$ on the world sheet imposes the eigenvalues of momentum to be quantized:

$$
p=\frac{k}{R}, \quad k \in \mathbb{N}
$$

For the modes $a_{n}$ canonical commutation relations imply the usual algebra

$$
\left[a_{n}, a_{m}\right]=\left[\bar{a}_{n}, \bar{a}_{m}\right]=n \delta_{m n}
$$

A representation of these algebras (2.13 and 2.16) is defined by

$$
\begin{gathered}
a_{n}|k l\rangle=\bar{a}_{n}|k l\rangle=0 \quad n>0 \\
a_{0}|k l\rangle=\frac{k}{R}+\frac{R l}{2}, \quad \bar{a}_{0}|k l\rangle=\frac{k}{R}-\frac{R l}{2}
\end{gathered}
$$

\subsection{Conformal Field Theory}

It is well known (and easy to see) that the action (2.10) has a huge number of symmetries:

$$
z \rightarrow f(z), \quad \bar{z} \rightarrow \bar{f}(\bar{z}),
$$

where $f$ is an arbitrary function. These are the conformal transformations in 2 dimensions. That makes the theory described by the aforementioned action a Conformal Field Theory. These numerous symmetries are one of the reasons why we can calculate so much in string theory. In the following we will need to allow for additional contributions to the action under the condition that they have conformal symmetry. In this section we will therefore study general features of conformal field theory in two dimensions.

We continue to the complex plane and treat $f$ and $\bar{f}$ independently. This of course implies that $z$ and $\bar{z}$ are no longer dependent and that we have enlarged our space from two real to two complex dimensions. This is all fine as long as in the end we remember that the physical space is the space where $z^{*}=\bar{z}$.

A tensor field with both $z$ end $\bar{z}$ indices would under these transformations transform as:

$$
\Phi_{p \times} \underbrace{}_{q \times} \ldots z \bar{z}(z, \bar{z}) \rightarrow\left(\frac{\partial f}{\partial z}\right)^{p}\left(\frac{\partial \bar{f}}{\partial \bar{z}}\right)^{q} \Phi_{z \ldots z \bar{z} \ldots \bar{z}}(f(z), \bar{f}(\bar{z})) .
$$

The numbers $p, q$ are called the conformal weights of a field.

The fact that the action is invariant under conformal transformations implies that the energy momentum tensor is traceless $\left(T_{a}^{a}=0\right)$. Together with the fact that the energy momentum tensor is a conserved current $\left(\partial_{a} T^{a b}=0\right)$ this translates into the following relations in $z, \bar{z}$ coordinates:

$$
\begin{aligned}
T_{z \bar{z}} & =T_{\bar{z} z}=0, \\
\bar{\partial} T_{z z} & =\partial T_{\bar{z} \bar{z}}=0 .
\end{aligned}
$$


In other words the energy momentum tensor has only two independent components, one holomorphic and one anti-holomorphic, which we will call $T$ and $\bar{T}$ respectively. The conserved Nöther currents that generate the (infinitesimal form of the) transformations (2.19) are easily seen to be

$$
\begin{aligned}
& J_{\epsilon}(z)=T(z) \epsilon(z), \\
& \bar{J}_{\bar{\epsilon}}(\bar{z})=\bar{T}(\bar{z}) \bar{\epsilon}(\bar{z}) .
\end{aligned}
$$

So all the conserved currents also split up in a holomorphic and anti-holomorphic part. This means that we can write down a Laurent expansion for these currents. In particular for $T$ :

$$
T(z)=\sum_{n} L_{n} z^{-n-2}
$$

As usual for each current we can construct a conserved charge by integrating the time component over space. Because on the plane space is a circle around the origin, this gives us contour integrals.

$$
Q_{\epsilon}=\frac{1}{2 \pi i} \oint d z \epsilon(z) T(z)
$$

These charges now become operators in the quantum theory. A convenient choice of basis for the functions $\epsilon(z)$ is $z^{n}$, with this choice our set of charges corresponding to the symmetries (2.19) becomes:

$$
Q_{z^{n+1}}=\frac{1}{2 \pi i} \oint d z z^{n+1} T(z)=L_{n}
$$

Now as we said the charges $L_{n}$ should generate the infinitesimal form of the transformations (2.19), this means that on a conformal field with weight $(h, \bar{h})$ it acts as:

$$
\delta \phi(z, \bar{z})=\left[L_{n}, \phi\right]=\left(h(n+1) z^{n}+z^{n+1} \partial\right) \phi(z, \bar{z})
$$

From this relation we can infer what the operator product between the energy momentum tensor and a conformal field should be. We can see these if we write out the commutator,

$$
\left[L_{n}, \phi(z, \bar{z})\right]=\frac{1}{2 \pi i} \oint d w w^{n+1}(T(w) \phi(z)-\phi(z) T(w)) .
$$

Here we make a judicious choice of contours. For the first term we choose a contour where $w>z$, for the second one with $w<z$. We can now deform the contour and end up with a integral of a radially ordered product over an contour circling $z$,

$$
=\frac{1}{2 \pi i} \oint_{(} d w z^{n+1} R\{T(w) \phi(z, \bar{z})\}
$$


If we want to reproduce transformation (2.28) the radial ordered operator product, as a series of $(w-z)$, has to have the following form:

$$
T(w) \phi(z, \bar{z})=\frac{h}{(w-z)^{2}} \phi(z, \bar{z})+\frac{1}{w-z} \partial \phi(z, \bar{z})+\ldots
$$

Note that we dropped the radial ordering symbol. While in the end we are always interested in correlation functions in the quantum theory, this will never cause confusion. Because, as usual in field theory, these correlation functions written in terms of operators, are always defined for time ordered products. And because time flows radially on the plane, time ordering becomes radial ordering.

With the canonical energy-momentum tensor for the scalar fields (2.2), one can calculate the operator product for the energy-momentum tensor with itself.

$$
T(w) T(z)=\frac{c / 2}{(w-z)^{4}}+\frac{2 T(z)}{(w-z)^{2}}+\frac{\partial T(z)}{w-z}+\ldots
$$

Note the appearance of the first term (which came out with $c=1$ for one scalar field). Without it $T$ would be a proper conformal primary field. As a side note this means there is a subtlety with the definition of the Hamiltonian. The Hamiltonian as defined as the integral over space of the $T_{\tau \tau}$ component on the world sheet will get an extra contribution, when we perform the map (2.9) from the cylinder to the complex plane. The result is not just the sum of zero modes of $T$ and $\bar{T}$ as we would expect for proper conformal fields, but with an additional term.

$$
H=L_{0}-\frac{c}{24}+\bar{L}_{0}-\frac{\bar{c}}{24}
$$

Using equation (2.32) to calculate the commutator of two of the operators (2.27), we find the Virasoro algebra:

$$
\left[L_{n}, L_{m}\right]=(n-m) L_{n+m}+\frac{c}{12} n\left(n^{2}-1\right) \delta_{n+m, 0}
$$

The last term in this equation is the so-called central term. If we would check the commutator of two infinitesimal transformations (2.28) classically this term would be absent. It is introduced by quantum effects and signals breaking of conformal symmetry. The factor of proportionality of this term $(c)$ is therefore called the conformal anomaly. The value of $c$ varies for the system under investigation; Free bosons add 1, free fermions $\frac{1}{2}$ and the reparametrization ghosts -26 .

We find of course a similar algebra for $\bar{L}_{n}$, and because $T$ is holomorphic (or chiral) and $\bar{T}$ anti-holomorphic (or anti-chiral) the operators $L_{n}$ and $\bar{L}_{n}$ commute. The symmetry algebra of the full theory is a product of the left chiral and ant-chiral algebra, which here are isomorphic.

Our Hilbert space should fall into representations of the algebra of these $L_{n} \mathrm{~s}$. We can build a realization of this algebra by starting from states satisfying:

$$
\begin{aligned}
\bar{L}_{n}|h, \bar{h}\rangle & =L_{n}|h, \bar{h}\rangle=0, \quad n>0 \\
L_{0}|h, \bar{h}\rangle & =h, \quad L_{0}|h, \bar{h}\rangle=\bar{h}
\end{aligned}
$$


The states $|h, \bar{h}\rangle$ have the maximal symmetry we can have. If we would also demand $L_{-n}|h, \bar{h}\rangle=0$, we would run into an inconsistency; From the commutator (2.34) with $m=-n$ one sees that a state that is both annihilated by $n$ and $-n$ will have vanishing left hand side but a finite right hand side through the central term. It follows from the algebra (2.34) that applying $L_{-n}$ always increases and $L_{n}$ decreases the $L_{0}$ eigenvalue. With the relations above it is then clear that the state $|h, \bar{h}\rangle$ has the minimal $L_{0}$ eigenvalue one can create by applying the operators $L$. Because $L_{0}+\bar{L}_{0}$ is proportional to the Hamiltonian, we have the nice property that the Hamiltonian is bounded from below; Precisely what we would demand from a physical system.

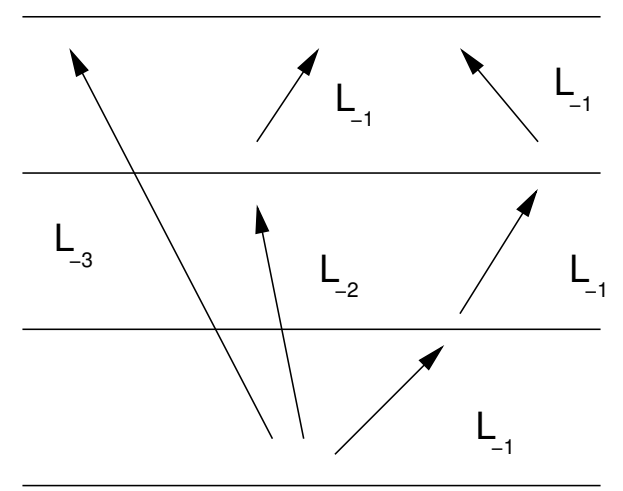

Figure 2.2: Virasoro Representation

The states $|h, \bar{h}\rangle$ are somewhat confusingly called highest weight states in the literature, we will also call them ground states. The rest of states in the representation that we can build on top of the ground states by applying the negatively moded operators are called descendants. Among the states $|h, \bar{h}\rangle$ the state with $h=\bar{h}=0$ has the most symmetry, it is also invariant under the $S L(2, C)$ sub algebra generated by $L_{0}$ and $L_{ \pm}$. We will call this state the vacuum, in sensible theories there is always one unique state satisfying this condition.

It can happen that for certain values of $c$ and $h$ one creates states with negative or zero norm. For the theory to make sense as physical theory these should not be present. It turns out that the former class can consistently be removed, while the later should not be allowed to exist in the first place; they give restrictions on for what values of $c$ and $h$ there exist consistent CFTs. For example for $0<c<1$ and $h \geq 0$, only the models with central term and primaries with conformal weight,

$$
\begin{gathered}
c=1-\frac{6}{m(m+1)}, \quad m \geq 3 \\
h=\frac{((m+1) p-m q)^{2}-1}{4 m(m+1)}, \quad 1 \leq p \leq m-1, \quad 1 \leq q \leq m
\end{gathered}
$$

satisfy all constraints. These models are called the $N=0$ or Virasoro minimal models. 
In this section we silently changed our subject from fields to states. The relation between the two can be made precise. If we consider a conformal field $\phi(z, \bar{z})$ with weights $(h, \bar{h})$ the connection is:

$$
|h, \bar{h}\rangle=\phi(0,0)|0\rangle
$$

By applying $L_{0}$ to this identity and using equation (2.29) (and similar for $\bar{L}_{0}$ ),

$$
\begin{aligned}
L_{0}|h, \bar{h}\rangle & =\left[L_{0}, \phi(0)\right]|0\rangle=h \phi(0)|0\rangle \\
& =h|h, \bar{h}\rangle,
\end{aligned}
$$

we see that $|h, \bar{h}\rangle$ is indeed a state with weights $(h, \bar{h})$.

\subsection{Simple currents}

Correlation functions in a conformal field theory are severely constrained by the large number of symmetries. For example the 1-point function can be shown to vanish and the two-point functions of two primary fields is fully constraint to take the following form:

$$
\left\langle 0\left|\phi_{1}\left(z_{1}, \bar{z}_{1}\right) \phi_{2}\left(z_{1}, \bar{z}_{1}\right)\right| 0\right\rangle=\left(z_{1}-z_{2}\right)^{-2 h_{1}}\left(\bar{z}_{1}-\bar{z}_{2}\right)^{-2 \bar{h}_{1}} \delta_{h_{1}, h_{2}} \delta_{\bar{h}_{1}, \bar{h}_{2}}
$$

Also correlation functions which includes descendants can always unambiguously given in terms of those of primaries. The full conformal field theory is not specified however, without the primary field content and the operator product expansion. In concrete conformal field theories there can be any number of primary fields. For example in the case of the boson of 2.2 every state $|p, \bar{p}\rangle$ labels another ground state. There is a class of conformal field theory, the rational conformal field theories, that have a finite number of primary fields. Obviously this makes concrete calculations much more tractable.

The operator product expansion states how one can replace two fields close to each other with a linear combination of all fields in the theory:

$$
\phi_{i \bar{\imath}}(z, \bar{z}) \phi_{j \bar{\jmath}}(w, \bar{w})=\sum_{k \bar{k}} C_{i \bar{\imath} j \bar{\jmath} k \bar{k}}(z-w)^{h_{k}-h_{i}-h_{j}}(\bar{z}-\bar{w})^{h_{\bar{k}}-h_{\bar{\imath}}-h_{\bar{\jmath}}} \phi_{k \bar{k}}(w, \bar{w})
$$

The coefficients $C_{i \bar{\imath} j \bar{j} k \bar{k}}$ are theory specific and known only for few theories. Fortunately sometimes one only needs to know if a certain field appears on the right hand side or not. The fusion rules summarize that information:

$$
[i] \otimes[j]=\sum N_{i j}{ }^{k}[k]
$$

this formula reads as follows: representation $k$ appears in the expansion of the product $i$ times $j$ if the coefficient is non-zero. With can determine theses coefficients with the help of the Verlinde formula, which give them in terms of the modular transformation matrices $S$ (see section 2.8).

A special type of fields are the so called simple currents. These are fields with particularly simple fusion rules; the fusion of simple current with any other field has only one field 
appearing on the right hand side. This implies that a additional fusion with the simple current would of course lead to, in general another, unique field. By associativity of the fusion rules the fusion of two simple currents is also a simple current. For rational theories there is a finite number of fusions before one reaches the identity map. This all means that the group of all simple currents (which is called the center) is a product of cyclic groups.

One can arrange all primaries in orbits under these cyclic factors. If for example we have a center which is $\mathbb{Z}_{N}$ and generated by $J$ we would have $N$ fields on an orbit that can be written as

$$
i, J \otimes i, J^{2} \otimes i, \ldots J^{N-1} \otimes i
$$

One useful fact about these orbits is that matrix elements of $S$ are related in a simple way for fields that lie on the same orbit. An important application is the construction of extended algebras, see 2.6.

\subsection{Open strings}

In the analysis so far we basically had closed strings in mind, for open strings we will have to do some more work. The usual route for open strings is to redo the work of the last section starting from an expansion for $X$ that satisfies the boundary conditions (2.11). We than find that there is only one set of modes $a_{n}$. Another approach, which lends itself much better for generalization and makes a clear connection between open and closed strings, is to view the boundary condition as constraints on fields living on the whole plane. In other words, by creating a boundary on the plane we can create a theory with open strings from one that contains only closed ones.

What we are going to do is to restrict the theory on the whole plane to one defined for $\operatorname{Im}(z)>0$. As we already discussed in 2.1, this means that holomorphic fields are defined on the upper half $z$-plane and anti-holomorphic field on the $\bar{z}$-lower. Let us, as an example, look at the currents $J$ and $\bar{J}$. While fields are living only on a half-plane it is hard to see how to define charges, that is until we see we can do a doubling trick. We now define a new object $\mathrm{J}$ defined on a plane that is just the upper half plane of $z$ glued to the lower half plane of $\bar{z}$.

$$
\mathrm{J}(z)= \begin{cases}J(z) & \operatorname{Im}(z)>0 \\ \pm \bar{J}(z) & \operatorname{Im}(z)<0\end{cases}
$$

The new field $\mathbf{J}$ is analytic in $z$ on the whole plane. This is obvious in the interior of the upper half plane because $J$ is and on the lower because $\bar{J}$ is. Around the real line it is also fine because we have the boundary conditions (2.11) on the real line. Let us for the moment continue only with Dirichlet boundary conditions, e.g. with the plus sign in (2.46), so we can not get confused by minus signs. Because $\mathrm{J}$ is analytic in $z$ we can write a Laurent expansion for its modes. Just as we did in previous sections these modes are conserved charges and defined as contour integrals:

$$
\mathrm{J}_{n}=\frac{1}{2 \pi i} \oint d z z^{n} \mathrm{~J}(z)
$$


and while $J$ is defined in terms of $J$ and $\bar{J}$ we can split the integral in one on the upper half plane and one on the lower half plane

$$
=\frac{1}{2 \pi i} \int_{n} d z z^{n} J(z)+\frac{1}{2 \pi i} \int_{\hookrightarrow} d z z^{n} \bar{J}(z)
$$

Finally because of the boundary condition (2.11) on the real line, we can close both contours

$$
=\frac{1}{2 \pi i} \oint d z z^{n} J(z)+\frac{1}{2 \pi i} \oint d \bar{z} \bar{z}^{n} \bar{J}(\bar{z})
$$

In the last line we renamed the integration variable form $z$ to $\bar{z}$ to represent the fact that the contour is entirely in the lower half plane. Notice that we have only one set of modes again, but the difference is that we know how they are defined in terms of closed string modes. We can, of course, do the same with the fields $T(z)$ and $\bar{T}(\bar{z})$. In the usual way we can find the form of correlation functions merely by imposing conformal symmetry. The trick goes as follows; we use the fact that both $\langle 0|$ and $|0\rangle$ are annihilated by $L_{i}$ for $i=0, \pm 1$, hence we find:

$$
\left\langle 0\left|\left[\mathrm{~L}_{i}, \phi(z, \bar{z})\right]\right| 0\right\rangle=\left(h(i+1) z^{i}+z^{i+1} \partial+\bar{h}(i+1) \bar{z}^{i}+\bar{z}^{i+1} \bar{\partial}\right)\langle\phi(z, \bar{z})\rangle=0
$$

From invariance under $L_{-1}$, we find that $\langle\phi(z, \bar{z})\rangle$ should be function of $(z-\bar{z})$, from $L_{0}$ that it should be of power $(-h-\bar{h})$ and finally from $L_{1}$ invariance that $h=\bar{h}$. In other words, we can see that the 1-point functions of conformal fields no longer have to vanish, they have the following form:

$$
\langle\phi(z, \bar{z})\rangle=\frac{\delta_{h \bar{h}}}{(z-\bar{z})^{2 h}}
$$

This is not too surprising given the fact that the boundary at $z=\bar{z}$ breaks some of the conformal symmetry.

\subsection{Additional Symmetries}

In general we will be interested in conformal theories that have additional symmetries. These symmetries are generated by the modes of conformal fields very much like the modes $L_{n}$ generate the conformal symmetry.

Conformal fields with weights $(h, 0)$ are necessarily holomorphic as can be deduced by looking at the norm of its first descendant state.

$$
\| \bar{L}_{-1}|h\rangle \|^{2}=\left\langle h\left|\left[\bar{L}_{1}, \bar{L}_{-1}\right]\right| h\right\rangle
$$

which together with the Virasoro algebra gives:

$$
=2 \bar{h} \||h\rangle \|^{2}=0
$$


and while $\bar{\partial} J|0\rangle=\bar{L}_{-1}|h\rangle$,

$$
\bar{\partial} J=0
$$

Hence the Laurent expansion of these fields exists, and its modes can be used to extend the chiral algebra. Because these fields are conformal, the commutator with one of the operators $L_{n}$ is precisely of the form (2.28). Filling in the expansion,

$$
J(z)=\sum J_{n} z^{n-h}
$$

Gives us the commutators:

$$
\left[L_{n}, J_{m}\right]=(n(h-1)-m) J_{n+m}
$$

The rest of the structure of the algebra, the commutators between the $J_{\mathrm{s}}$, differs case by case.

Representations of this extended algebra are built on ground states that are now annihilated by the positive modes $L_{n}$ and $J_{m}$. It is easy to see that applying an operator $J_{m}$ changes the conformal weight of a state by $m$. The total symmetry algebra still contains the Virasoro algebra as a sub-algebra. This means that representation of this algebra can always be decomposed in representations of the Virasoro algebra. Looking from it the other way: extending the Virasoro algebra, groups Virasoro representations together in representations of the extended algebra thereby reducing the number of ground states. This reduction can be dramatic; In certain extensions an infinite number of Virasoro representations get grouped together, thereby making the number of ground states finite. This makes the number of independent correlation functions suddenly much smaller, making chances of solving the theory much greater.

An example of an extension is the boson described in section 2.2. In that section the Virasoro structure is not really apparent, however a small derivation shows that the Virasoro modes can be expressed as $L_{n}=\frac{1}{2} \sum_{m} a_{n-m} a_{m}$. We made the right choices for some factors in (2.12) to display nicely that the modes $a$ are actually the modes of two (anti-)holomorphic currents:

$$
\begin{aligned}
& J(z)=i \partial X=\sum_{n} a_{n} z^{-n-1} \\
& \bar{J}(\bar{z})=i \bar{\partial} X=\sum_{n} \bar{a}_{n} \bar{z}^{-n-1}
\end{aligned}
$$

The commutators they add to the algebra are (were the modes $a_{n}$ are renamed to $J_{n}$ ):

$$
\begin{gathered}
{\left[L_{n}, J_{m}\right]=-m J_{m+n}} \\
{\left[J_{m}, J_{n}\right]=m \delta_{m+n}}
\end{gathered}
$$

Another example of an extended algebra is the $N=2$ super conformal algebra. It can be realized with one complex boson and fermion pair on the world sheet. The algebra contains 
apart from the Virasoro generators, the modes of a $U(1)$ current like the previous example and the modes of two anti commuting currents $G^{ \pm}$:

$$
\begin{gathered}
\left\{G_{r}^{+}, G_{s}^{-}\right\}=2 L_{r+s}+(r-s) J_{r+s}+\frac{c}{3}\left(r^{2}-\frac{1}{4}\right) \delta_{r+s} \\
{\left[J_{m}, G_{r}^{ \pm}\right]= \pm G_{r}^{ \pm} .}
\end{gathered}
$$

A series of rational conformal field theories, the unitary $N=2$ Virasoro models exist with conformal anomaly,

$$
c=3\left(1-\frac{2}{m}\right), \quad m \geq 3 .
$$

These models are attractive to work with, because explicit constructions exist, they will provide the building blocks for the CFTs that will give standard model like vacua in the next chapter.

The last example that really should be mentioned is that of extending the chiral algebra with simple currents of integral conformal weight already present in a CFT. It can be used to conveniently create new CFTs from existing ones. By choosing appropriate simple currents it is possible to remove unwanted states. As explained in section 2.4 simple currents group primaries into orbits. The extension projects out the states from some orbits. The remaining orbits each provide a character of the new theory as a linear combination of the old characters, also the modular $S$-matrix can be derived from that of the parent theory. Sometimes it happens that, although $J^{m} \neq 0$ there exists fields that are mapped to themselves $J^{m} \otimes f=f$, such fields are called fixed points. The orbits that contains $f$ is necessarily shorter, this gives problems in finding the $S$ matrix. To be able to use the formalism one has to resolve the fixed points [71, 129]. Basically one has to find an extra label, that splits up the field $f$ in enough copies to complete the orbit. The so called fixed point resolution matrix is then needed to construct the $S$-matrix of the extended theory from the one of the parent.

\subsection{Algebraic constructions}

In the last section we investigated some general two dimensional conformal field theory. We will now try to make the connection with string theory again.

A general CFT will have a conformal anomaly. As noted, this signals the breakdown of conformal symmetry in the quantum theory. This will, as with gauge anomalies, show up as severe problems which differ as one changes the way one approaches the problem; loss of unitarity, inability to couple to gravity, etc. In other words; in a consistent string theory the conformal anomaly needs to vanish. Fortunately because the conformal anomaly is additive one can always add several sectors together to get a consistent theory.

As alluded to before, to properly treat the gauge choice made for the world sheet metric one has to introduce ghosts. As it turns out these reparametrization ghosts form a conformal field theory (bc-CFT) with $c=-26$. Because every boson on the world sheet $(X)$ adds one to conformal anomaly, we found the root of the famous statement "Strings live in 26 dimensions". This statement has been revised when Superstrings were 
discovered. In Super string theory one adds non-commuting fields $\Psi$ to the string theory action (2.3) that are world sheet super partners of the bosons $X$. These fields $\Psi$ each add $c=\frac{1}{2}$. The symmetry algebra is now enlarged from conformal to super-conformal. This also means we need extra gauge fixing ghosts. The CFT that can be used for that, $\beta \gamma$-CFT, has conformal anomaly 11. With some simple arithmetic we then find we have to add 10 super-space coordinates.

There has been various routes to try to get this rather big number of dimensions more in-line with every day experience. One way is through geometric constructions. This encompasses for example rolling up these extra dimensions (choosing periodic boundary conditions) to a size which is much smaller than can be currently detected. From the field theory perspective, this means that the index on the fields $X$ and $\Psi$ that enumerates the dimension splits up in 0 through 3 , which is our well known space time, and the other directions, which label some other internal sector. Generally this approach can only be followed in regimes where geometry is essentially classical; curvature is not too large and quantum gravity effects can be neglected. This has the advantage that one can leverage the intuition one has for geometry.

A different approach, the one primarily used in this thesis, is the algebraic approach. It is geared to taking full advantage of the symmetry algebra of the two dimensional world sheet. In the algebraic approach one does not specify where the internal CFT comes from. Apart from some other consistency conditions one just demands that the total conformal anomaly adds up to zero:

$$
c_{\text {internal }}=-4 c_{X}-4 c_{\psi}-c_{\text {ghost }}=9
$$

The strength of this approach is that it is valid for all distance scales. Another advantage comes from the fact that the symmetry group underlying these theories highly constrains various quantities which makes them easily calculable.

The class of models we will use as internal sector to construct phenomenologically interesting string vacua (four dimensional ones with one supersymmetry) go under the name of Gepner models. Gepner models are tensor products of $N=2$ minimal models that were briefly introduced in section 2.6. Because we are limited to tensor products with conformal anomaly $c=9$, there is finite number of ways one can combine the minimal models. Classifying all possibilities is a simple exercise. In table 4.2 one finds all 168. This does not mean that taking the Gepner models as starting point one can only study 168 models. In fact most of them have a non-trivial group of simple currents, which can be used to extend the chiral algebra and build simple current invariants (see section 4.2 and $[113,134,78])$. Apart from these simple current invariants some more invariants are known to exist. Unlike for the simple current invariants, there is no general procedure that can be used to find them and build open string theories from them.

In the following section we will go in to more detail how to construct a consistent closed string theory, and from that open string theories. 


\subsection{Partition Functions}

If we were to calculate the closed string theory amplitude for state $|a\rangle$ scattering into state $|b\rangle$, the amplitude is defined as a sum over all processes that can connect these two states; similar to amplitudes in quantum field theory. The Feynman diagrams of point particles with vertices and lines are replaced by strings propagating, splitting and recombining. The series in number of loops in QFT is roughly replaced by a series in the number of handles in the surface swept out by the strings. Just as in QFT one sums over all nonequivalent processes, which sometimes means integrating over a free parameter. In order not to over-count one has to be careful to divide out symmetries of the theory. Here conformal symmetry for example helps to reduce the number of diagrams; All field theory diagrams with equal number of loops in field theory map to a string diagram with the same topology.

By again using the freedom to reshape the world sheet one can map the in and outgoing states to insertions of closed two dimensional surfaces. The series for a $n$-point amplitude thus changes to a sum over surfaces with increasing genus each with $n$ insertions; Starting with the sphere, then a torus, a two-torus and so forth.

Under conformal symmetry transformations all surfaces with the topology of a sphere can be mapped in each other, leaving only one inequivalent diagram at tree level. Higher genus surfaces usually have parameters that enables one to distinguish them. Let us consider the first string loop vacuum diagram: the torus.

$$
\mathcal{T}=\int_{\forall T^{2}} \mathcal{D} X e^{-S[X]}
$$

To really make sense of this integral one has to be more careful by for example introducing ghosts. We will take a more heuristic approach, for a more in depth treatment see [116].

First different tori which have locally not 'smooth' can be made smooth by using the conformal symmetry. Then this torus can be defined as the complex plane with periodic identifications in both the real and imaginary direction. We now want to determine which fundamental domains that span this lattice describe inequivalent tori. This lattice can always be rotated and scaled such that one of the basis vectors has length $2 \pi$ and is aligned with the real axis. The remaining basis vector, $2 \pi \tau$, then labels the different tori. It is called the modulus of torus. If $\tau$ is purely imaginary the torus is 'straight', the norm of $\tau$ measures the ratio of the length of the two cycles. If $\tau$ has a real component, the torus is 'twisted'. Obviously if it is twisted by $2 \pi$, we are back at the 'straight' torus.

$$
T: \tau \rightarrow \tau+1
$$

Another redundancy, maybe a bit less obvious, is the transformation:

$$
S: \tau \rightarrow-\frac{1}{\tau}
$$

it interchanges the two basis vectors with an appropriate rescaling to again get a torus with $\operatorname{Re} z=2 \pi$. It amounts to saying that the labelling of the axis is arbitrary. If we look 
carefully what happens we have exchanged worldsheet space and time, but also flipped the time direction, hence doing this twice reverses both time and space. We will have to apply $S$ four times to get the identity map. However $S^{2}$ does bring the modular parameter back to itself. It is part of the conformal symmetry of the torus. If we consider extended symmetries $S^{2}=C$ can work as an involution, on the fields. $C$ is called the charge conjugation matrix, the charge conjugate of a field $i$ in denoted as $i^{c}$, they have equal conformal weight. Together with the symmetry $\tau \rightarrow-\tau$ the transformations $S$ and $T$ make up the group of transformations that leave the torus invariant, the modular group. This group is $S l(2, \mathbb{Z}) / \mathbb{Z}_{2}$, the group of two dimensional linear transformations with integer coefficients where $\pm \tau$ are identified.

The path integral is an integral of all allowed field configuration of $X$ on all tori, can thus be done by integrating over all inequivalent $\tau$.

$$
\mathcal{T}=\int \frac{d \tau}{\operatorname{Im}^{2} \tau} \int_{T^{2}(\tau)} \mathcal{D} X e^{-S[X]}
$$

The factor $\operatorname{Im}^{2} \tau$ in the measure comes in from properly taking care of ghosts.

The path integral over $X(z, \bar{z})$ is like quantum mechanics for a scalar, but now not only propagating for $2 \pi i \operatorname{Im} \tau$ in a 'time' direction, but also along the string for a distance $2 \pi \operatorname{Re} \tau$ in a 'space' direction. And because of the periodic boundary conditions, we can write the path integral as a trace over all states of the unitary operator that generates this evolution,

$$
\begin{aligned}
\mathcal{T}(\tau, \bar{\tau}) & =\operatorname{Tr} e^{-2 \pi \operatorname{Im}(\tau) H+2 \pi i \operatorname{Re}(\tau) P} \\
& =\operatorname{Tr} e^{2 \pi i \tau\left(L_{0}-\frac{c}{24}\right)} e^{-2 \pi i \bar{\tau}\left(\bar{L}_{0}-\frac{\bar{c}}{24}\right)}
\end{aligned}
$$

where in the last line we used (2.33) for $H, P$ can be found similarly. The last expression in (2.71) makes only reference to the operators $L_{0}$ and $\bar{L}_{0}$, which are be available for any CFT. This is the correct expression for the torus-partition function for a general conformal field theory.

For later convenience we rewrite it to:

$$
\begin{aligned}
& \mathcal{T}(\tau, \bar{\tau})=\sum_{i, j} \chi_{i}(\tau) Z_{i j} \chi_{j}(\bar{\tau}), \\
& \chi_{i}(\tau)=\operatorname{Tr}_{\text {Rep }(i)} e^{2 \pi i\left(L_{0}-\frac{c}{24}\right)} \tau .
\end{aligned}
$$

The sum over $i, j$ is over all Virasoro representations; all ground states. For the rational conformal field theories we are interested in this is a finite sum, for an uncompactified boson this would be an integral over momentum. Note that for the free boson which we implicitly used to derived the torus parition function, $Z$ is just the identity matrix here. The quantity $\chi$ is called the Virasoro character of representation $i$; the trace is over all (non null) states in that representation. As we have seen in section 2.3, $L_{0}$ is an integer giving the level of excitation of a state and $\frac{c}{24}$ measures the difference between the level 
and the 'mass' of the state. If we write $q=e^{2 \pi i \tau}$ and denote the ground state 'mass' as $h_{i}$ we can expand the characters as:

$$
\chi_{i}(q)=q^{h_{i}-\frac{c}{24}} \sum_{n} d_{n} q^{n}
$$

The coefficients $d_{n}$ are naturally integers, they count the number of states at level $n$.

As we have seen the torus should be modular invariant, meaning that the group of transformations generated by (2.67) and (2.68) should be a symmetry. The characters are not invariant under the transformation. It is easy to see that that under $T$ the characters acquire a phase. With some more work one can show that also $S$ maps characters to a linear combination of the original characters. We can thus represent both transformations as matrices,

$$
\begin{aligned}
& \chi_{i}(\tau+1)=\sum_{j} T_{i j} \chi_{j}(\tau) \\
& \chi_{i}\left(-\frac{1}{\tau}\right)=\sum_{j} S_{i j} \chi_{j}(\tau),
\end{aligned}
$$

where $S_{i j}$ turns out to be a symmetric unitary matrix.

For the integrated torus to be invariant under this transformation, we need

$$
[T, Z]=[S, Z]=0 .
$$

This formula of course trivially holds for the identity matrix, but writing it in this way enables us to find new modular invariant partition functions from a given conformal field theory. All we have to do, to find all of them, is classify all possible integer matrix (because the entries denotes the number of states at a certain level), that commutes with the matrices $S$ and $T$. In addition we demand $Z_{00}=1$, because we want the vacuum to be a unique state.

\subsubsection{Unoriented Strings}

String theory as introduced in section 2.1 has an additional discrete symmetry we overlooked so far. It is the reversal of the orientation of the world sheet. Changing the parametrization of the world sheet by $\sigma \rightarrow 2 \pi-\sigma$ (or $z \rightarrow \bar{z}$ ) should not matter for the physics. In other words, the map $\Omega$ that implements this transformation works as follows:

$$
\Omega(X(\sigma))=X(2 \pi-\sigma)
$$

If we reverse the orientation twice, we are back at the original orientation $\left(\Omega^{2}=1\right)$; Which means that the eigenvalues of this map are \pm 1 . For the closed string theory we studied, we can deduce from the expansion (2.12) that the this map should swap the oscillator sets:

$$
\Omega a_{n} \Omega^{-1}=\bar{a}_{n}
$$


Linear combinations like $a_{n}+\bar{a}_{n}$ are thus symmetric under this map. To fix how this map works on the full set of states, we define the primary states $|k, \bar{k}\rangle$ to be symmetric; $\Omega|k, \bar{k}\rangle=|k, \bar{k}\rangle$. In theories with more structure more general eigenvalue assignments for the ground states are possible. The possible choices for these assignments are related to possible choices of $K_{i}$ below in (2.83) (see chapter 3 for examples and more details).

We can now build new unoriented closed string theories from oriented ones by projecting out states that are anti-symmetric under orientation reversal. The partition function of such a theory is easily written down by including a projection operator in the trace (2.71)

$$
\begin{array}{r}
\operatorname{Tr} \frac{1+\Omega}{2} e^{2 \pi i \tau\left(L_{0}-\frac{c}{24}\right)} e^{-2 \pi i \bar{\tau}\left(\bar{L}_{0}-\frac{\bar{c}}{24}\right)} \\
=\frac{1}{2} \mathcal{T}(\tau)+\frac{1}{2} \mathcal{K}(t)
\end{array}
$$

Taking the trace is a linear operation, so the partition function splits up in the original torus partition function and a second term. From (2.79) we learn that in the second term, only terms with equal $L_{0}$ and $\bar{L}_{0}$ eigenvalue will survive.

$$
\operatorname{Tr} \Omega e^{-2 \pi \operatorname{Im}(\tau) H_{\text {closed }}}
$$

Note that the trace here is still over the closed string spectrum. Translating this trace back into a path integral, one can interpret this expression as the path integral of a closed string propagating with periodic boundary condition for a time $2 \pi i \operatorname{Im} \tau$ (which we will write with $t=\operatorname{Im} \tau$ ), but coming back with orientation reversed. The unorientable surface such a closed string would sweep out is the famous Klein bottle. It can be drawn in three dimensions (let alone projected to two), but it looks something like in picture 2.3. The

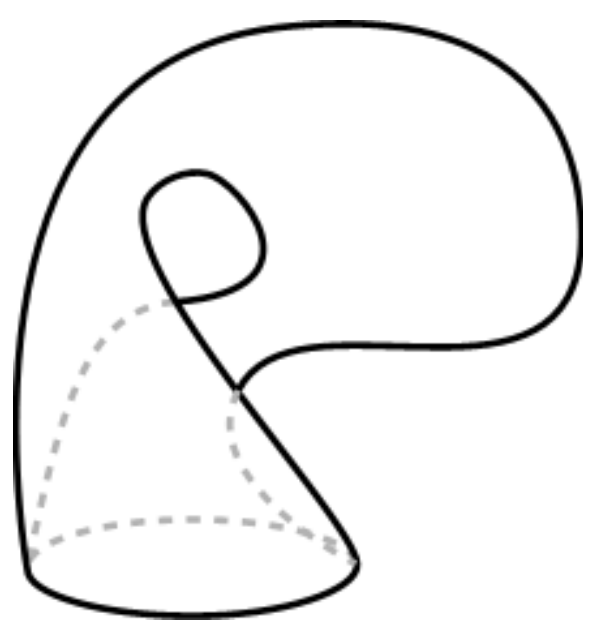

Figure 2.3: Klein bottle

modulus of this surface is one dimensional; unlike the torus it has no continuous twist we can apply while gluing the ends together with change of orientation. 
Comparing with (2.73), we see that in terms of characters the Klein bottle is linear. We can read of the argument of the character as $2 i t$. The factor two is because (2.82) includes both $L_{0}$ and $\bar{L}_{0}$, which are equal for all states that contribute.

$$
\mathcal{K}(t)=\sum_{i} K^{i} \chi_{i}(2 i t)
$$

\subsubsection{Annulus \& Möbius strip}

Often unoriented closed strings are not consistent by themselves, the Klein bottle leads to divergencies. These divergencies can usually be cancelled by introducing an open string sector. In similar vein as done for closed strings one can calculate the one loop partition function for open strings. Again we have to use periodic time. To have the same time period as with the closed string case we choose it to have a period $2 \pi t$

$$
\begin{aligned}
\mathcal{A}(t) & =\operatorname{Tr} e^{-2 \pi t H_{\text {open }}} \\
& =\operatorname{Tr} e^{-\pi t\left(L_{0}-\frac{c}{24}\right)} \\
& =\sum_{i} A^{i} \chi_{i}\left(\frac{i t}{2}\right)
\end{aligned}
$$

Just as for closed strings we can divide out orientation reversal to obtain an unoriented theory. Orientation reversal for the open string means $\sigma \rightarrow \pi-\sigma$, which in its turn maps

$$
\Omega a_{m} \Omega^{-1}=(-1)^{m} a_{m}
$$

To be consistent with the expansion the map $\Omega$ working on

$$
\begin{aligned}
& \operatorname{Tr} \frac{1+\Omega}{2} e^{-2 \pi t H_{\text {open }}} \\
& =\frac{1}{2} \mathcal{A}(t)+\frac{1}{2} \mathcal{M}(t)
\end{aligned}
$$

The partition function $\mathcal{M}(t)$ describes an open string propagating for a time $2 \pi t$ and coming back with orientation reversed. The unorientable surface that is swept out by the string, is that of the Möbius strip.

If we want to write the partition function in terms of characters we notice that the minus sign in (2.87) (which of course can be written as $e^{\pi i m}$ ) introduces a shift in the characters argument.

$$
\mathcal{M}(t)=\sum_{i} M^{i} \chi_{i}\left(\frac{i t+1}{2}\right)
$$

\subsection{Open descendants}

In section 2.8 we found we could find consistent closed string theories by searching for matrices $Z$. As the title of thesis suggests we would however want to construct open 
unoriented string theories. Fortunately there exists a method by which we can use the torus partition functions found in the last section to construct such theories. It is know as finding the open descendants of a closed string theory. The resulting theory is then called an Orientifold. For that we first need the notion of boundary states.

\subsection{Boundary States}

We are interested in a theory that describes both open and closed strings, therefore we are looking for a description of open strings in terms of closed string operators. For that we will have a closer look at the one loop open string vacuum diagram, the equivalent of the torus for open strings. It is an open string with periodic time condition and hence has the shape of a cylinder. Conformally it is equal to an annulus (disk with a hole in it) and that is what it is usually called in the literature. This picture could equally well depict a

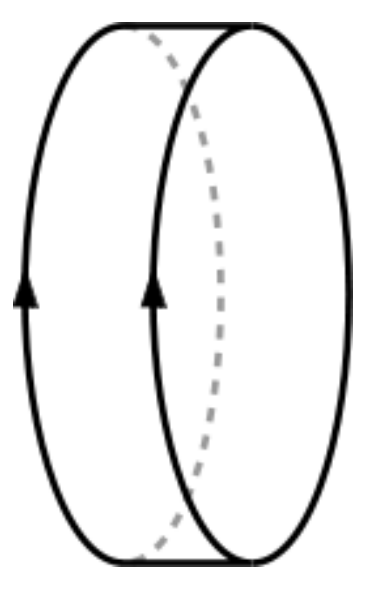

Figure 2.4: One loop open string vacuum diagram

closed string emitted by something, propagating for a while and then annihilated again, only the world sheet time runs in the wrong direction. But that we can fix by a conformal transformation. In a schematic formula:

$$
\mathcal{A}(t)=\left\langle B_{b}\left|e^{i t H_{\text {closed }}}\right| B_{a}\right\rangle
$$

The state $\left|B_{a}\right\rangle$, is a called a boundary state, it represent the something that emits or annihilates a closed string, in string theory they are called D-branes, the ' $\mathrm{D}$ ' for Dirichlet and 'brane' from membrane. As drawn in figure 2.4 these objects are planes, but if the strings live in more dimensions they can be a hypersurface of any dimension.

\subsection{Channel transformations}

Just like the annulus has different channels, the Möbius strip and Klein bottle do too. The correspondence is a bit harder to read from a simple drawing of a Klein bottle. Analysing 
how you can make one of these surfaces from a torus by identification makes things much clearer. Let us start with the annulus; it can be made from a torus with purely imaginary modulus $\tau=i t$ by identifying points by:

$$
z \simeq-\bar{z}
$$

It amounts to identifying points with its image reflected through the line $\operatorname{Re} z=\pi$. By the periodic condition, the lines $\operatorname{Re} z=0, \pi$ are identified with themselves, they are fixed points of the identification. These lines are boundaries, you can not move past them. Moving below $\operatorname{Re} z<0$ for example is by the identification equivalent to moving $\operatorname{Re} z>0$.

The fundamental region of the original torus covers that of the annulus twice. A fundamental domain of the annulus is the region:

$$
0 \leq \operatorname{Re} z \leq \pi, \quad 0 \leq \operatorname{Im} z \leq 2 \pi t
$$

With time running in the imaginary direction, this corresponds to an open string propagating with periodic boundary conditions with period $2 \pi t$, stretched between two boundaries at $\operatorname{Re} z=0, \pi$ respectively. The modulus of the annulus is the real parameter $t$. All annuli with different $t$ are inequivalent, there is no modular group of transformations that leave the annulus invariant.

There is a subgroup of the modular group on the double cover, the torus, that respects the moduli space of the annulus, namely the transformation (2.68); it will map the imaginary axis to itself. Performing the conformal transformation $S$, the region (2.92) gets mapped to the region

$$
0 \leq \operatorname{Re} z \leq 2 \pi, \quad 0 \leq \operatorname{Im} z \leq \pi / t .
$$

As expected, this changes world sheet time and space, with a rescaling such that 'space' of the covering torus has again a circumference of $2 \pi$. The boundaries are now surfaces of equal time at $\operatorname{Im} z=0, \pi / t$, the space direction is again periodically identified; it is a closed string that is created at some time, propagates for a world sheet time $\pi / t$ and then ceases to exist.

The Klein bottle can be constructed from a torus with purely imaginary modulus $2 \pi i t$ by identifying points by:

$$
z \simeq-\bar{z}+\pi i t
$$

This identifies points reflected through the line $\operatorname{Re} z=\pi$ but now while translating 'time' by half the period. To have a clearer picture what this means we study what happens with the boundaries of the fundamental region. A fundamental region of the Klein bottle is:

$$
0 \leq \operatorname{Re} z \leq 2 \pi, \quad 0 \leq \operatorname{Im} z \leq \pi t
$$

In the space direction the edges of this region are periodically identified; it is a closed string. In the time direction there is also a periodic identification, but after a reflection in $\operatorname{Re} z=\pi$, after a change in orientation. Performing the transformation $S$ on the map, now has the result that we have to identify after a reflection in $\operatorname{Im} z=\pi / t$ and translation of 


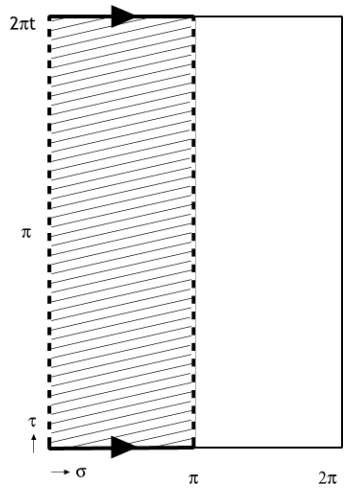

(a) Direct

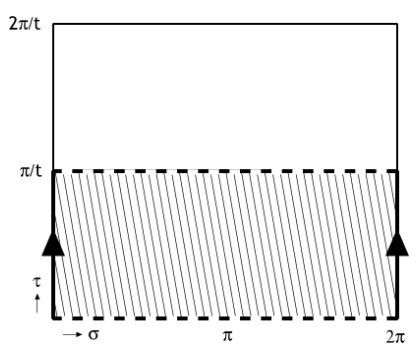

(b) Transverse

Figure 2.5: Annulus

Re $z$ by $\pi$. Of course the fundamental region (2.95) is also rotated. It turns out however to be more intuitive to not look at the rotated image, but at the equally fundamental region

$$
0 \leq \operatorname{Re} z \leq 2 \pi, \quad 0 \leq \operatorname{Im} z \leq \pi / t .
$$

Again in the space direction the edges are identified periodically. At world sheet time $\operatorname{Im} z=0, \pi / t$ however, the string is identified with itself after a translation of half its length. This is what one calls a cross-cap. The Klein bottle can be interpreted as a closed string propagating for a world sheet period $\pi / t$ between two cross-caps.

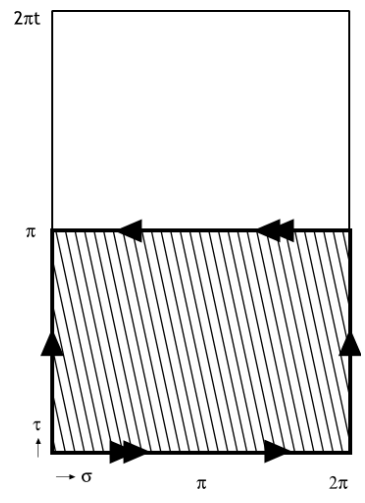

(a) Direct

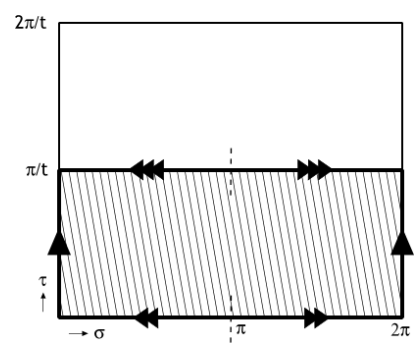

(b) Transverse

Figure 2.6: Klein bottle

The last surface we need to consider is the Möbius strip. It can be constructed from a torus with modulus $\tau=i t+\frac{1}{2}$ by identifying:

$$
z \simeq-\bar{z}
$$


A fundamental region is the strip:

$$
\pi \leq \operatorname{Re} z \leq 2 \pi, \quad 0 \leq \operatorname{Im} z \leq 2 \pi t
$$

The bottom edge of this strip $0 \leq \operatorname{Re} z \leq \pi$ is via the reflection through $\operatorname{Im} z=\pi$ and the torus identification identified with the top edge after orientation reversal. Both edges $\operatorname{Re} z=\pi, 2 \pi$ are identified with themselves by the reflection and the torus identification $z=z+2 \pi$; these are again boundaries. We can interpret this region as an open string propagating for a time $t$ coming back with reversed orientation. Note that following the boundary $\operatorname{Re} z=\pi$ past time $2 \pi t$ we end up via he identification $z \simeq z+2 \pi \tau$ on the boundary $\operatorname{Re} z=2 \pi$; this surfaces has only one boundary, Just as one would expect from a Möbius strip.

The simple transformation $S$, does not respect the moduli space of the Möbius strip. It turns out that the transformation $T S T^{2} S$ does. It is also somewhat harder to see the closed string interpretation, it is easiest to look at the region in picture $2.7(\mathrm{~b})$. Following

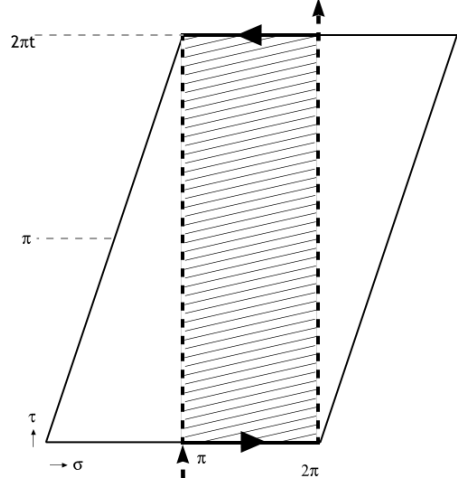

(a) Direct

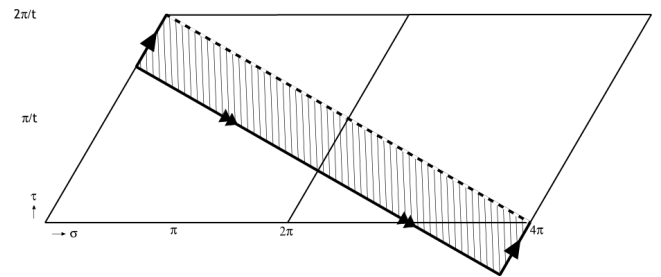

(b) Transverse

Figure 2.7: Möbius strip

the identifications one sees that the top line is mapped to itself; it is a boundary. The bottom line is identified with itself after translation by half its length; it is a cross-cap. In the 'space' direction the edges are identified periodically with orientation reversal. This is closed string propagating between a boundary and a cross-cap.

\subsection{Ishibashi states}

The boundary conditions (2.11) on the real line for the fields $\partial X$ and $\bar{\partial} X$ translate into $T(z)=\bar{T}(\bar{z})$ for the energy momentum tensor. If the theory contains more symmetries than those generated by $T$ we will have to specify boundary conditions for those too. To be able to incorporate them into a conformal field theory on the plane, in other words, perform the doubling trick 2.5, we demand that the boundary conditions are at least an 
automorphism of the chiral algebra. On the cylinder we write this as:

$$
J(z)=\omega(\bar{J}(\bar{z}))
$$

To formally incorporate these boundary conditions in the conformal field theory the boundary states need to satisfy those conditions as operator equations. We should perform a transformation on the boundary condition, that exchanges the world sheet space and time variables, so that we can interpret it as a closed string process, we will rescale the new space variable to the standard length $2 \pi$. Because we want to derive these conditions for the states $|B\rangle$ that live on the complex plane, we will at the same time map the cylinder to the plane with the usual transformation. This leads us to the transformation:

$$
(\tau+i \sigma) \rightarrow e^{\frac{2 \pi i}{\beta}(\tau+i \sigma)}
$$

For a conformal field with weight $h$ and a time period of length $\beta$, this gives us on the plane the following condition on the currents:

$$
\begin{gathered}
(i z)^{h} J(z)-(-i \bar{z})^{h} \omega(\bar{J}(\bar{z}))=0 \\
\text { at } \bar{z}=z^{-1}
\end{gathered}
$$

Using the relation between fields and states this leads us to

$$
\left[J_{n}-(-1)^{h} \omega\left(\bar{J}_{-n}\right)\right]|B\rangle=0
$$

A non-trivial example of such conditions are precisely the Dirichlet and Neumann conditions on the currents $\partial X$ and $\bar{\partial} X$. On the modes of these chiral current, the conditions take the form:

$$
\left[a_{n} \mp \bar{a}_{-n}\right]|B\rangle=0,
$$

where the only non-trivial automorphism for that chiral algebra is $\omega\left(a_{n}\right)=-a_{n}$.

The solutions to the equations (2.103) are called Ishibashi states. In general writing down explicit solutions is hard. One can however write down formal expressions in terms of the conformal primaries [99]. These expressions are enough to calculate the amplitudes we want, namely

$$
\left\langle\left\langle i\left|e^{-\pi 2 \pi t H_{\text {closed }}}\right| j\right\rangle\right\rangle=\delta_{i j} \chi_{i}(4 i t)
$$

The boundary states that we can add to our theory are linear combinations of Ishibashi states.

$$
\left.\left|B_{a}\right\rangle=\sum_{i} B_{a i}|i\rangle\right\rangle
$$

The numbers $B_{a i}$ are called boundary coefficients. These coefficients are important quantities. As we will see, they can be used to determine the spectrum of the string theory. To find the boundary coefficients one should solve the sewing constraints, but for this one needs to know the so called Fusion matrices of the full CFT. These matrices are not 
yet available for the CFTs we are interested in. Fortunately the boundary coefficients are severely constrained by the so called positivity and integrality conditions $[125,40]$ for which one does not need to know these matrices.

In the free boson case it is not so hard to find the explicit solutions. For the Dirichlet case for example the states are of the form

$$
|k\rangle\rangle=e^{\sum_{n>0} \frac{1}{n} a_{-n} \bar{a}_{-n}}|k\rangle \bigotimes|k\rangle
$$

The Ishibashi states are thus coherent states of closed string states, formally they are not part of the closed string Hilbert space.

The cross-cap lives at $\bar{z}=-z^{-1}$, so the analogous condition to (2.103) for cross-cap states becomes:

$$
\left[J_{n}-(-1)^{h+n} \omega\left(\bar{J}_{-n}\right)\right]|C\rangle=0 .
$$

For an unoriented theory we have a second set, the cross-cap Ishibashi states. Again what we really care for are amplitudes of closed strings propagating between Ishibashi states. To get a complete set we have to supplement (2.105) with:

$$
\begin{array}{r}
{ }_{c}\left\langle\left\langle i\left|e^{-2 \pi t H_{\text {closed }}}\right| j\right\rangle\right\rangle_{c}=\delta_{i j} \chi_{i}(2 i t) \\
{ }_{c}\left\langle\left\langle i\left|e^{-2 \pi t H_{\text {closed }}}\right| j\right\rangle\right\rangle_{b}=\delta_{i j} \sqrt{T} \chi_{i}\left(2 i t+\frac{1}{2}\right),
\end{array}
$$

where $\sqrt{T}$ is just a diagonal matrix with half the phases that enters in the modular $T$ matrix. This turns out to be convenient later on.

\subsection{Transverse channel}

As we have seen in section 2.11 on page 27 , we can map vacuum diagrams of unoriented and open strings to diagrams involving only closed strings that are emitted and annihilated by boundaries and cross-caps. One can allow for multiple copies of the same boundary present in a theory. The Chan-Paton multiplicity, $N_{a}$ counts this number. The total boundary state can then be written as:

$$
\left.|B\rangle=\sum_{i, a} N_{a} B_{a i}|i\rangle\right\rangle
$$

A similar multiplicity does not exist for cross-cap states because one can only consistently add one cross-cap state at same time, just like one can only have one orientifold projection. With this notation we can write down the relations between the transverse diagrams and the corresponding unoriented and open string partition functions as follows:

$$
\begin{aligned}
\mathcal{A} & =\int d t\left\langle B\left|e^{2 \pi i t H_{\text {closed }}}\right| B\right\rangle \\
\mathcal{M} & =\int d t\left\langle B\left|e^{2 \pi i t H_{\text {closed }}}\right| C\right\rangle+\langle C|\ldots| B\rangle \\
\mathcal{K} & =\int d t\left\langle C\left|e^{2 \pi i t H_{\text {closed }}}\right| C\right\rangle
\end{aligned}
$$


By evaluating the right hand sides with the help of the expressions (2.105) and (2.109) we found for the Ishibashi states, we end up again with expressions in terms of characters similar to those we found in section 2.7. We still have to perform the channel transformations and to be able to compare with those expressions a change of variables is needed. When that is done we arrive at set of relations between boundary and cross-cap coefficients and the coefficients $A_{a b}^{i}, M_{a}^{i}$ and $K^{i}$. The annulus coefficients for example are given by:

$$
A_{a b}^{i}=\sum_{m} S_{m}^{i} B_{m a} B_{m b}
$$

The left hand side of this equation are parts of the coefficients in the partition function of an unoriented open string,

$$
\frac{1}{2}(\mathcal{T}+\mathcal{K})+\frac{1}{2}(\mathcal{A}+\mathcal{M})
$$

and as such are restricted by the fact these coefficients are state multiplicities. Taking for example the open sector, $(A+M) / 2$ and filling in the expansion of the characters, we can see that the number of states at level $h_{i}+n-1$ stretched between boundary $a$ and $b$ is given by:

$$
\frac{1}{2}\left(N_{a} N_{b} A_{a b}^{i} \pm N_{a} M_{a}^{i}\right) d_{n}^{i}
$$

From this we can deduce that $A_{a b}^{i} \in \mathbb{Z}^{+}$. This and similar constraints go under the name of positivity an integrality conditions. Because $B_{i a}$ and $S_{m}^{i}$ are in general complex numbers, it is clear that these constraints imply highly non-trivial constraints on the boundary coefficients. Fortunately for a large class of CFTs (namely all MIPFs that are a simple current extension of a charge conjugation invariant) it is known [69] how to construct consistent cross-cap and boundary coefficients from closed string data. This means we know how to construct unoriented open string theories, open descendants from a closed parent theory. Further consistency conditions still have to be satisfied to avoid local (see sec. 4.4) and global anomalies, this can usually be done by choosing appropriate ChanPaton factors. 


\section{Chapter 3}

\section{On Orientifolds of $c=1$ Orbifolds}

\subsection{Introduction}

The $\mathrm{c}=1$ orbifold CFTs on closed, oriented Riemann surfaces have been studied extensively [58][57][84][104] because they provide simple but non-trivial examples of various features of conformal field theory. It has long been believed that all $\mathrm{c}=1$ bosonic theories in the closed oriented case were known: they either belonged to the famous continuous moduli space of the circle and its $Z_{2}$ orbifold, or one of three discrete points discovered in [84]. The completeness proof [104] was based on certain assumptions, and more recently counterexamples have been conjectured to exist [123]. The open, unoriented case has received considerably less attention. Early results can be found in [120], reviewed recently in [8], and there are some remarks on such theories in the appendix of [39]. Our purpose here is a systematic study of the set of theories that is obtained if one allows open and unoriented surfaces. We will study this problem for continuous values of the radius $R$ and at rational points, and match the results.

For the circle theories the solution to this problem is known. On oriented, closed surfaces the moduli space is a line parametrized by the radius $R$ of the circle, with a Tduality identification of $R$ and $\alpha^{\prime} / R$. On unoriented surfaces this line splits into two lines. In one of the two T-dual pictures the two lines are characterized by having O0 planes of either the same tension or opposite tension on opposite points of the circle, in the other the circle is covered by $\mathrm{O} 1$ planes. This matches precisely and unambiguously with the results from rational CFT, as we will see in slightly more detail below.

Studying this problem on the orbifold line is interesting for a variety of reasons: the set of modular invariant partition functions (MIPFs) is richer, there is an interesting one-toone map between the rational $\mathrm{c}=1$ orbifolds at radius $R^{2}=\alpha^{\prime} N$ and the $D_{N}$ WZW-models at level 2 [128] and the orbifolds have a twist field degeneracy [57] which is resolved only in the rational points. Finally we are interested in understanding the puzzling results of [131], showing that in certain rational points the orbifold CFT does not admit a canonical Klein bottle projection (with all coefficients equal to 1 ). There are non-canonical solutions, but it is not immediately clear how they would fit into a continuum description. 
Two types of approaches to boundary RCFT are used in this paper. In [69] a general formula was given for all reflection coefficients and crosscap coefficients for all simple current modular invariants [110] and a large class of orientifold projections. This formula is consistent (at least in the sense of yielding integral coefficients, see [69] and [96]; furthermore consistency for all resulting oriented amplitudes was demonstrated recently in [73]), but there is no proof that it is complete. In [132] a set of polynomial equations was written down which can be solved for annulus, Moebius and Klein bottle coefficients. This method is complete, but it is not known if all solutions are physical. Our goal is to do a systematic analysis of all known orbifold RCFTs and compare them with the continuum, to check if the method of [69] misses anything, and if the method of [132] produces anything manifestly unphysical. The orbifolds are an ideal laboratory for this because the continuum can be obtained explicitly, and RCFTs are known in all rational points. The circle has all these features as well, but lacks some non-trivial structure related to the fixed points, as well as exceptional modular invariants.

A prerequisite for this work is a complete description of orientifolds in the continuous case. Although there is a large body of work of orientifolds of tori and orbifolds, as far as we know the complete and explicit answer for the $c=1$ orbifolds is not available yet, although a partial result can be found in [8]. We find a total of four different allowed Klein bottle amplitudes and corresponding orientifold projections.

The structure of the paper is the following. In Section 2 we review the results concerning the bosonic string on a circle and the orientifolds thereof. In Section 3.3 we consider the orbifold case. First, in subsection 3.1, we study orientifolds for rational radius. A parametrization of the allowed Klein bottles in terms of RCFT characters leads to twelve different choices. Then we enumerate the modular invariant partition functions (MIPFs), by systematically combining all known types and checking closure under multiplication. For each MIPF we perform a systematic construction of U-NIMreps [135] (U-NIMreps are sets of Annulus, Möbius and Klein bottle amplitudes that satisfy the aforementioned polynomial equations; they are NIMreps [55][119][20][19] extended with data concerning unoriented surfaces.) This method is limited in practice to a finite number of primaries, but we can extend it far enough to uncover the complete picture, and furthermore we supplement it with the formula of [69], which has no such limitation. We conclude that for integer values of $R^{2} / \alpha^{\prime}$ six different cases can be distinguished, corresponding to four distinct continuum Klein bottle amplitudes. As a result of these computations we obtain a set of boundary and crosscap coefficients which we use in subsection 3.1.4 to study the localization of branes and O-planes, as well as the Chan-Paton groups in the various cases. In subsection 3.2 we study the case of orientifolds at continuous radius from a geometric point of view, and confirm that the four Klein bottle amplitudes are indeed the only possible ones. In subsection 3.3 we consider the case of certain non-integer rational radii (exceptional MIPFs) and show that, despite their unexpected features, the results of [131] are precisely in agreement with the four orientifold projections. In Section 4 we summarize our conclusions. In the appendix we discuss a variety of orbifold maps needed to obtain distinct orbifold theories that exist in the rational case. 


\subsection{Summary of circle results}

In this section we want to review know results about orientifolds of the bosonic string on a circle, in order to set up the discussion for the orbifold case and to introduce some notations. Orientifolds of circle compactifications for irrational values of the radius appeared for the first time in [23], while additional references are [51][7][139][75][22][52] (see [8] for a review). The partition function for a bosonic closed oriented string compactified on a circle is

$$
Z(R)=\frac{1}{\eta \bar{\eta}} \sum_{m, n} q^{\frac{\alpha \prime}{4}(m / R+n R / \alpha \prime)^{2}} \bar{q}^{\frac{\alpha^{\prime}}{4}(m / R-n R / \alpha \prime)^{2}},
$$

where we denote with $n$ the winding and $m / R$ the Kaluza-Klein (KK) momentum along the circle. A well known feature of this partition function is the fact that it is invariant under the exchange $R \leftrightarrow \alpha^{\prime} / R$. From the point of view of the extended CFT that describes the above theory, the partition function $Z(R)$ hides chiral information. For each value of $R$ there are actually two theories with the same partition function, which are each others T-dual, one with momentum states $\left|\sqrt{\frac{\alpha \prime}{2}}(m / R+n R / \alpha \prime), \sqrt{\frac{\alpha \prime}{2}}(m / R-n R / \alpha \prime)\right\rangle$, and one with states $\left|\sqrt{\frac{\alpha \prime}{2}}(m / R+n R / \alpha \prime), \sqrt{\frac{\alpha \prime}{2}}(-m / R+n R / \alpha \prime)\right\rangle$. The first of these is obtained in a genuine compactification on a circle of radius $R$.

The allowed orientifold projections must respect the operator product expansion of the CFT (we will only consider orientifold projections of order 2). This implies in particular that the operator $\partial X \bar{\partial} X$ must be transformed into itself, and that the vertex operators corresponding to the momentum and winding states must transform with a factor $\left(\epsilon_{1}\right)^{m}\left(\epsilon_{2}\right)^{n}$, with $\epsilon_{1}$ and $\epsilon_{2}$ equal to \pm 1 . Hence one can associate four consistent Klein bottles with this partition function, namely ${ }^{1}$

$$
\begin{gathered}
\frac{1}{\eta\left(2 i \tau_{2}\right)} \sum_{m} q^{\frac{\alpha \prime}{2}(m / R)^{2}} \equiv 2 K_{+00}(R) \\
\frac{1}{\eta\left(2 i \tau_{2}\right)} \sum_{m}(-1)^{m} q^{\frac{\alpha \prime}{2}(m / R)^{2}} \equiv 2 K_{-00}(R) \\
\frac{1}{\eta\left(2 i \tau_{2}\right)} \sum_{n} q^{\frac{1}{2 \alpha^{\prime}}(n R)^{2}} \equiv 2 K_{0+0}(R) \\
\frac{1}{\eta\left(2 i \tau_{2}\right)} \sum_{n}(-1)^{n} q^{\frac{1}{2 \alpha^{\prime}}(n R)^{2}} \equiv 2 K_{0-0}(R) .
\end{gathered}
$$

The functions $K$ will be introduced later. The Klein bottle amplitude is subject to the constraint that $\frac{1}{2}(Z(R)+K(R))$ expands into non-negative integers. The first two Klein bottles can be combined with the diagonal theory, the other two with its T-dual. The

\footnotetext{
${ }^{1}$ We assume here that the relation between the orientifold projection and the Klein bottle is straightforward. This implies in particular that the Klein bottle coefficients are preserved under fusion, i.e that the "Klein bottle constraint" is satisfied. There are examples where this constraint is not satisfied (see $e . g$ [95]) and which require further thought, but this problem does not occur for any of the c=1 U-NIMreps.
} 
positions of the orientifold planes can be derived from the transverse channel Klein bottle amplitudes by dimensional analysis. In the first two cases the transverse amplitude describes a closed string propagating between two O1 planes. There are two different O1 planes. In the first case, the resonance term permits only to even winding states to propagate in the transverse channel, while in the second case only odd winding states contribute, and thus the configuration has vanishing tension, since the graviton does not propagate in the transverse channel. We call these two configurations $\mathrm{O} 1_{+} \oplus \mathrm{O} 1_{+}$and $\mathrm{O} 1_{+} \oplus \mathrm{O} 1_{-}$ respectively. In the first case, the orientifold projection maps $X$ to itself, while in the second case it maps $X$ to $X+\pi R$. Both these maps square to the identity because of the periodicity of the circle, and have no fixed points. The other two cases are the T duals of the former. After $\mathrm{T}$ duality, the orientifold projection acts as a $Z_{2}$ orbifold on the circle coordinate, so that the model lives on a segment, with $\mathrm{O} 0$ planes at the endpoints. In the third case, the orientifold projection maps $X$ to $-X$, while in the fourth case it maps $X$ to $-X+\pi R$. Both these projections have fixed points, where the O0-planes are located. More precisely, the third case corresponds to the configuration $\mathrm{O} 0_{+} \oplus \mathrm{O} 0_{+}$, in which the two O-planes have the same tension, while the fourth case corresponds to $\mathrm{O} 0_{+} \oplus \mathrm{O} 0_{-}$, in which the O-planes have opposite tension.

The corresponding rational CFTs are obtained by setting $R^{2}=\alpha / N$. All primaries of this CFT are simple currents, forming a $Z_{2 N}$ discrete group. We denote the generator of $Z_{2 N}$ as $J$. All MIPFs are simple current invariants, and they are in one-to-one correspondence with the subgroups of $Z_{2 N}$ generated by even powers of $J$, which in their turn are in one-to-one correspondence with the divisors of $N$. If $m$ is a divisor of $N$, the MIPF belonging to the subgroup generated by $J^{2 m}$ corresponds to a circle with radius $R^{2}=\alpha / N / m^{2}$. We will refer to this partition functions as $Z_{\text {circle }}(m, N)$, with the convention that $Z_{\text {circle }}(N, N)$ is the charge conjugation invariant and $Z_{\text {circle }}(1, N)$ the diagonal one. For every rational radius $R^{2}=\alpha \prime p / q$ (with $p$ and $q$ relative prime) there is an infinite number of rational CFTs describing it, namely $Z\left(p r, p q r^{2}\right)$, for any $r \in \mathbf{Z}$. For $r>1$ the corresponding partition functions involve extensions of the chiral algebra.

For all these MIPFs the allowed crosscap and boundary coefficients follow from the general formula presented in [69] (summarizing and extending earlier work in [93, 74, 76, $77,94,95]$ ), which in the special case $Z_{N, N}$ reduces to the well-known boundary state of Cardy [40] combined with the crosscap state due to the Rome group [118].

By Fourier analyzing the closed string scattering amplitudes from the boundary and crosscap states (a procedure that was pioneered in [56], and applied in [67] to boundary states of WZW models and in [92] to crosscap states (see also [37][15])) one can localize the D-branes and O-planes on the circle. To do this one multiplies the boundary and crosscap amplitude with a factor $e^{i k x / R}$ and sums over all values of $k$ in the primary range $-N \leq k<N$. The resulting function of $x$ has peaks that get more pronounced with increasing $N$, and are interpreted as the brane and plane positions. ¿From the continuous point of view, we expect D1 branes for the diagonal invariant (corresponding to a genuine circle compactification, with the space-filling brane wrapped around the circle), which turn into D0 branes for the charge conjugation invariant, the T-dual of the former.

In the rational CFT one finds the following. The MIPF $Z(m, N)$ admits $2 m$ boundaries, 
each of which is localized at $n=N / m$ distinct points on the circle. To make sense of this we introduce the dual radius $\hat{R}=\alpha \prime / R$, which is the relevant quantity because D0 branes live on the dual circle. The $n$-fold multiplicity is an indication of the fact that the original circle of dual radius $\hat{R}$ is to be interpreted as an $n$-fold cover of a circle of radius $\hat{R} / n$. We may label the boundaries by integers $a=0, \ldots, m-1$, such that they are localized at points $\left(\frac{a+2 m \ell}{2 N}\right) 2 \pi \hat{R}, \ell=0, \ldots, n-1$. For $m=N, n=1$ (the charge conjugation invariant) this yields $2 N$ D0 branes equally distributed over the circle; for $m=1, n=N$ (the diagonal invariant) this gives two branes localized simultaneously on $N$ equally distributed points, one brane on the odd points and one on the even points. This is the RCFT realization of a D1 brane.

In the continuum the D0 can be localized anywhere on the circle, whereas in the rational CFT description their positions are quantized. One can approach the continuum results either by using deformations of the boundary CFT [122], or by allowing boundaries that break some of the extended symmetries that characterize the rational CFT. This can be done by obtaining the circle at some dual radius $\hat{R}$ from a circle at dual radius $\hat{R} r$ by extending the CFT of the latter. The RCFT notion of "completeness of boundaries" [119], when applied to MIPFs of extension type, automatically implies the presence of boundaries that break the extended symmetries. Indeed, if we consider $Z\left(p r, p q r^{2}\right)$ we find $2 p r$ distinct boundaries, each localized in $q r$ points on the circle of radius $\hat{R} r$. This circle is an $r$-fold cover of the circle of radius $\hat{R}$, so that on the latter circle we now have $2 p r$ distinct branes each localized in $q$ points. Of these, $2 p$ coincide with the ones found for $r=1$, and the remaining ones occupy intermediate positions. In the limit $r \rightarrow \infty, \hat{R}$ fixed we approach the continuum result.

Similar results hold for crosscaps. The formalism of [69] allows two ways of modifying the crosscap coefficient for a given MIPF. The formula for the crosscap coefficient is, up to normalization

$$
\Gamma_{i} \propto \sum_{L \in G} \eta(K, L) P_{K L, i}
$$

where $i$ is the Ishibashi label, $P$ the $P$-matrix $\left(P=\sqrt{T} S T^{2} S \sqrt{T}\right)$. Here $K$ is a simple current (subject to a condition given below), $\eta(K, L)$ a set of signs satisfying the constraint

$$
\eta(k, L)=e^{\pi i\left(h_{K}-h_{K L}\right)} \beta_{K}(L)
$$

where $\beta_{K}(L)$ is a set of phases solving the relation

$$
\beta_{K}(L J)=\beta_{K}(L) \beta_{K}(J) e^{-2 \pi i X(L, J)},
$$

where $X$ is the rational bihomorphism that specifies the MIPF, as defined in [110]. This relation does not fix the phases completely: for every independent even cyclic factor of the simple current group $G$, there is a free sign. These free signs are called the "crosscap signs". The current $K$ (for historical reasons called the "Klein bottle current") must be local w.r.t. the currents of order two in $G$, and currents that differ by elements of $G$ or by squares of simple currents yield equivalent crosscaps. These Klein bottle currents form, together with the crosscap signs, the set of allowed crosscap modifications. 
In the present case, it is not hard to see that for each choice of $G$ there are just two solutions. If $G$ has even order, there is a single crosscap sign choice, but there are no Klein bottle currents local w.r.t. $G$. If $G$ has odd order $n$ there is no crosscap sign choice, but then the current $J^{n}$ is local w.r.t. $G$ and is a non-trivial Klein bottle current. These two choices correspond precisely to the two orientifold choices in the continuous case. In all cases one of the orientifold choices leads to Klein bottle coefficients that are equal to +1 for all fields appearing diagonally.

The crosscap positions can be worked out in the same way as for D-branes. For the MIPF $Z(m, N)$ we find that a crosscap state occupies $2 n$ positions, twice as many as a boundary state. These positions are $n$-fold identified. In the simplest case, $m=N, n=1$ there are two positions, diametrically opposite on the circle. In this case, the crosscaps are characterized by the choice of Klein bottle current $K=J^{k}$. Each $k$ corresponds to two O0 planes localized at $r=\frac{k}{4 N} 2 \pi R$ and $r=\left(\frac{k+2 N}{4 N}\right) 2 \pi R$. If $k+N$ is even these two O0 planes have the same tension, if $k+N$ is odd they have opposite tension. If $k$ is even, the O-plane locations coincide with a brane position; if $k$ is odd the O-planes lie between two brane positions. Configurations where $k$ differs by an even integer can be obtained from each other by rotating the circle, in agreement with the fact that the corresponding Klein bottle currents are equivalent. The T-dual configuration corresponds to the diagonal invariant, obtained by using the simple current $J^{2}$. This MIPF admits just two Ishibashi states, and hence only two non-vanishing crosscap coefficients. This is not sufficient to contain any information about localization, in agreement with the fact that we expect the O-planes to be O1 planes wrapping the circle (and analogously for boundaries).

\subsection{Orbifolds}

We are considering the $c=1$ case, that is the real line modded out by the group $G$ of reflections and translations, resulting in the segment $\mathbb{R} / G=S^{1} / \mathbb{Z}_{2}$. Following [88] we will denote the action of elements of this group on the string coordinate as

$$
(\theta, n) \in G, \quad(\theta, n) X=\theta X+2 \pi n R, \quad n \in \mathbb{Z}, \theta= \pm .
$$

Strings on an orbifold are closed if they are periodically identified up to an element of this group:

$$
X(\sigma+2 \pi)=(\theta, n) X(\sigma) .
$$

$X$ is then twisted by the element $(\theta, n)$; this defines various sectors with different periodicity conditions on $X$. Not all elements of $G$ give rise to a different sector, a sector twisted by $g$ being the same as the one twisted by $h g h^{-1}$. Thus we get a sector for each conjugacy class. One has the following conjugacy classes: $(+, \pm|m|),(-$, even $)$ and $(-$, odd). The first gives the circle periodicity conditions with winding number $m$, where now the winding direction is no longer significant. The last two cases give the twisted sectors. Note that in these sectors the notion of winding is limited to being 'even' or 'odd'.

In the untwisted sector, $X$ has the same mode expansion as in the circle, with the difference that now only the states that are invariant under the map $X \rightarrow-X$ are present. 
Denoting with $r$ the operator that performs this map on the Hilbert space, the resulting spectrum is obtained by applying the projector $\frac{1+r}{2}$ on the circle states.

In the twisted sectors the mode expansion for $X$ is

$$
X=x_{o}+\sum_{n} \frac{1}{n-\frac{1}{2}}\left(a_{n-\frac{1}{2}} z^{-n+\frac{1}{2}}+\bar{a}_{n-\frac{1}{2}} \bar{z}^{-n+\frac{1}{2}}\right),
$$

where $x_{o}=0$ for the $(-$, even $)$ sector, $x_{o}=\pi R$ for the $(-$,odd $)$ sector. The twisted sectors correspond thus to states localized at the fixed points of the orbifold. Also the states created by the modes in (3.8) must be projected by the operator $\frac{1+r}{2}$.

The resulting partition function is

$$
Z_{\text {orb }}(R)=\frac{1}{2} Z_{\text {circle }}(R)+\left|\frac{\eta}{\theta_{2}}\right|+\left|\frac{\eta}{\theta_{3}}\right|+\left|\frac{\eta}{\theta_{4}}\right|
$$

The first two are contributions of the untwisted sectors, while the last two are contributions of the twisted sectors.

\subsubsection{Orientifolds for rational radius}

\section{Parametrizations of the Klein bottle}

In the CFT description, the allowed orientifold projections are limited by the requirement of preservation of the OPE. Of most interest are the projection signs of states appearing diagonally, since those signs affect the Klein bottle. Again we find that $\partial X \bar{\partial} X$ must transform into itself. This implies that $\frac{\eta}{\theta_{2}}$ cannot appear in the Klein bottle expression, since this function represents the difference of contributions of the identity operator and the operator $\partial X \bar{\partial} X$. The OPE of $\partial X \bar{\partial} X$ with a lowest weight twist field $\sigma(z, \bar{z})$ yields the excited twist field $\tau(z, \bar{z})$. Since $\partial X \bar{\partial} X$ has projection sign + , the twist fields $\sigma$ and $\tau$ must be projected in the same way. This implies the absence of $\frac{\eta}{\theta_{3}}$, which corresponds to the difference of the two twist field labels.

An important issue is twist field degeneracy. The $c=1$ orbifold has two twist fields (stemming from the fact we have two different twisted sectors), denoted $\sigma_{1}$ and $\sigma_{2}$ (with weight $\left(\frac{1}{16}, \frac{1}{16}\right)$ ) and two excited twist fields, $\tau_{1}$ and $\tau_{2}$, with weight $\left(\frac{9}{16}, \frac{9}{16}\right)$. The labels 1 and 2 are not distinguished by the Virasoro algebra. On any point on the orbifold line the Virasoro algebra is extended by operators that are even polynomial in $\partial X$ and its derivatives, the first one at weight 4 [57]. But these operators do not distinguish the labels either, since $\partial X$ itself does not. Only in the rational points there are operators that distinguish the twist fields, namely the operators $\cos \left(\sqrt{\frac{2}{\alpha \prime}} m R X\right)$ that extend the chiral algebra to make the CFT rational. Hence we should regard these as states with multiplicity 2. The allowed Klein bottle coefficients in this sector are the 2,0 or -2 . The value 0 is allowed if the twist fields appear off-diagonally in the partition function, or if they appear diagonally, but have opposite Klein bottle projections. Based on this information 
we arrive at the following twelve choices for the Klein bottle

$$
K_{\epsilon_{1} \epsilon_{2} \epsilon_{3}}=\frac{1}{2} \frac{1}{\eta\left(2 i \tau_{2}\right)} \sum_{k \in \mathbf{Z}}\left(\epsilon_{1}\right)^{k} q^{\frac{\alpha \prime}{2}\left(\frac{k}{R}\right)^{2}}+\frac{1}{2} \frac{1}{\eta\left(2 i \tau_{2}\right)} \sum_{m \in \mathbf{Z}}\left(\epsilon_{2}\right)^{m} q^{\frac{1}{2 \alpha \prime}(m R)^{2}}+2 \epsilon_{3} \sqrt{\frac{\eta}{\theta_{4}}},
$$

with $\epsilon_{1}= \pm 1 ; \epsilon_{2}= \pm 1$ and $\epsilon_{3}=0, \pm 1$. The same parametrization can be used for the circle theory, provided one allows the value 0 for $\epsilon_{1}$ and $\epsilon_{2}$.

As was the case for the circle, the allowable Klein bottles depend on the interpretation of the partition function. But in contrast to the circle theory, this interpretation is discontinuous in $R$. This is due to the fact that we can distinguish the twist fields only for rational $R$. In addition, for rational $R$ two orbifold fields appear that do not exist for irrational values of $R$, namely the fields we denote as $\phi_{1}$ and $\phi_{2}$ and that have conformal weight $\frac{1}{4} N$.

\section{Enumeration of modular invariants}

We will now study the orbifold at rational points in order to reduce the set of orientifold projections. In the rational points and for a sufficiently small set of primaries we have an additional tool at our disposal, namely the systematic search for NIMreps and U-NIMreps.

The orbifold of the circle of radius $R^{2}=\alpha / N$ (or its T-dual) is the well-known orbifold rational CFT with $N+7$ primaries. It has four simple currents, $1, \phi_{1}, \phi_{2}$ and the spin1 current $\partial X$, forming a discrete group $\mathbf{Z}_{4}$ (for $N$ odd) or $\mathbf{Z}_{2} \times \mathbf{Z}_{2}$ (for $N$ even). The remaining fields will be denoted $\varphi^{k}, k=1, \ldots, N_{1}, \sigma_{i}, \tau_{i}(i=1,2)$, following [57]. A lot is known about the MIPFs of these CFTs, but the result are scattered throughout the literature, and for that reason we will give here an enumeration of what is known.

In [57] it was observed that the theory at radius $R^{2}=\alpha \prime p / q$ or $R^{2}=\alpha \prime q / p$ has the same chiral algebra as the one at $R^{2}=\alpha \prime p q$. Hence it is described by a non-trivial MIPF of the theory at $R^{2}=\alpha \prime p q$. This MIPF is of exceptional type, except when $q$ and/or $p$ is equal to 2 , in which case the invariant is of simple current type. Just as in the circle case, one can generalize this by allowing $p$ and $q$ to have common factors. In this way one can obtain an infinite number of rational CFT realizations at any rational point on the orbifold line. Any of these MIPFs can be extended by the simple current $\partial X$ to re-obtain the circle partition function.

But there are still more rational partition functions for every rational point, a fact that can most easily be appreciated by using the fact that the modular group representation of the orbifold CFT for $R^{2}=\alpha / N$ is in one-to-one correspondence with the $D_{N}$ WZW model at level 2 [128]. In particular there is a one-to-one relation for partition functions, NIMreps and U-NIMreps. While the aforementioned MIPFs describing orbifolds at noninteger radii do not seem to have a raison d'etre for the WZW-models, they do exist for these models as well. The ones of automorphism type were discovered using Galois symmetry in [70] (subsequently the WZW automorphisms were fully classified in [79]); the extensions were described in [128] using the aforementioned correspondence. On the other hand, the $D_{N}$ WZW models (at any level) have MIPFs related to Dynkin diagram 
automorphisms that imply the existence of related invariants for the orbifolds. These are first of all the conjugation invariants, which have an off-diagonal pairing of $\phi_{1}, \phi_{2}, \sigma_{1}, \sigma_{2}$ and $\tau_{1}, \tau_{2}$. For odd $N$ this is the charge conjugation invariant, which can also be described as a simple current invariant generated by $\phi_{1}$ (or, equivalently, $\phi_{2}$ ). For even $N$ this is an exceptional invariant (for even $N$, the simple current invariant generated by $\phi_{1}$ gives an orbifold at reduced radius $N / 4$ ). If the MIPF involves an extension by $\phi_{1}$ or $\phi_{2}$ (which happens if $p$ and $q$ are both even), there are still more possibilities, because one can then conjugate the left and right chiral algebras independently. As a result one obtains two symmetric and two asymmetric (heterotic) MIPFs. Finally, for $N=4$ there are even more MIPFs related to triality of $D_{4}$; there are 16 MIPFs in total.

For given $N \neq 4$ the number of known modular invariants, obtained by combining all the above, can be described as follows. Let $p$ be a divisor of $N$ in the range $1 \leq p \leq \sqrt{N}$, and define $q=N / p$. The number of known invariants is equal to $\sum_{p} M(p)$ where the sum is over all divisors $p$ in this range, and $M(p)=3$ if either $p$ or $q$ is odd, $M(p)=5$ otherwise. The multiplicity three corresponds to the diagonal invariant, the conjugation invariant and the circle extension, which we denote respectively as $Z_{D}(p, N), Z_{C}(p, N)$ and $Z_{X}(p, N)$. The multiplicity five corresponds to the four cases described above plus the circle extension, denoted respectively as $Z_{11}(p, N), Z_{22}(p, N), Z_{12}(p, N), Z_{21}(p, N)$ and $Z_{X}(p, N)$ (the circle extension includes both $\phi_{1}$ and $\phi_{2}$ ).

Since the standard orbifold map $X \rightarrow-X$ yields just one orbifold theory for each T-dual pair of circle theories, one has to consider more general orbifold maps to get the various types of orbifold partition functions. These maps are discussed in the appendix.

\section{U-NIMreps}

Consider first $Z_{C}(1, N)$ and $Z_{D}(1, N)$. In all but one case these MIPFs are C-diagonal or of simple current type, and a set of Klein bottle coefficients can be obtained from various previous papers. To deal with the remaining exceptional MIPF $\left(Z_{C}(1, N), N\right.$ even), and as a check on all the others, we solved the U-NIMrep polynomial equations (for $N \leq 16$ ) to get the complete answer. This results in the following six cases:

1. The diagonal invariant, with standard Klein bottle $\left(K_{i}=1\right.$ for all $\left.i\right)$. For $N$ even, this is the standard Cardy-Rome case. For $N$ odd, it is a simple current automorphism, as treated in [69], with suitable choice of the crosscap sign. The resulting Klein bottle amplitude is $K_{+++}$. This result was first obtained in [120] (see also [8]).

2. The diagonal invariant, with Klein bottle coefficients -1 in the twisted sector. For $N$ even, this is the same as the previous case but with Klein bottle current 2. For $N$ odd, it is the same as the previous case, but with the opposite crosscap sign. The resulting Klein bottle is $K_{++-}$.

3. The diagonal invariant, with Klein bottle currents $\phi_{1}$ or $\phi_{2}$. This case exists only for $N$ even. For $N$ odd, the diagonal invariant is generated as a simple current automorphism of current $\phi_{1}$, and the only allowed sign changes are the crosscap 
signs, which we already saw above. For even $N$ one finds that all odd charged fields $\varphi^{k}$ get a negative Klein bottle, i.e. $K_{\varphi^{k}}=(-1)^{k}$. Furthermore either $\sigma_{1}$ and $\tau_{1}$ or $\sigma_{2}$ and $\tau_{2}$ change sign, so that the total twisted sector contribution cancels. The result is $K_{-+0}$ (for $N$ even only).

4. The charge conjugation invariant with standard Klein bottle ( i.e. all $K_{i}=+1$ if $i$ appears diagonally). The charge conjugation invariant only differs from the diagonal one for $N$ odd. The effect is that $\phi_{1}, \phi_{2}$ appear off-diagonally, and the same for the twisted sector. The latter implies $\epsilon_{3}=0$. The absence of $\phi_{1}, \phi_{2}$ in the Klein bottle amplitude implies $\epsilon_{2}=-1$, so that the contribution of $\phi_{i}$ cancels between the first two terms. Hence we get $K_{+-0}$ (for $N$ odd only).

5. The charge conjugation invariant with non-standard Klein bottle (case 4 with simple current Klein bottle current $\phi_{1}$, which is equivalent to $\phi_{2}$ ). This gives a sign flip for all odd charge fields, implying $\epsilon_{1}=-1$ This can be taken into account by inserting a $(-1)^{k}$ into the first sum. Since $N$ is odd, the $\phi_{i}$ contribution cancels between the first two terms if and only if $\epsilon_{2}=+1$. Hence we get $K_{-+0}$ (for $N$ odd only).

6. The conjugation invariant for even $N$. This is an exceptional invariant that pairs $\phi_{1}$ with $\phi_{2}, \sigma_{1}$ with $\sigma_{2}$ and $\tau_{1}$ with $\tau_{2}$. Here [69] does not apply, but by solving the NIMrep conditions explicitly we find only one NIMrep with one U-NIMrep. The Klein bottle has all allowed coefficients equal to 1 . This yields $K_{+-0}$ (for $N$ even only).

We summarize these results in the following table. In the first column ' $\mathrm{D}$ ' denotes the diagonal invariant, ' $\mathrm{C}$ ' the charge conjugation invariant, and ' $\mathrm{T}$ ' the twist field conjugation invariant, in which $\phi_{i}, \sigma_{i}$ and $\tau_{i}$ are off-diagonal. In the fifth column we indicate the ChanPaton group for the dominant branes (i.e. the ones that are most numerous for large $N$ ). This will be explained in the next subsection. The last column refers to the six cases listed above.

\begin{tabular}{|l|c|c|c|c|c|}
\hline Invariant & $N$ & Boundary/Crosscap formula & Klein bottle & CP-group & case \\
\hline $\mathrm{D}$ & odd & {$[69]$} & +++ & $S O$ & 1 \\
& & & ++- & $S O$ & 2 \\
\hline $\mathrm{C}=\mathrm{T}$ & odd & Cardy/Rome & +-0 & $S O$ & 4 \\
& & & -+0 & $U$ & 5 \\
\hline $\mathrm{D}=\mathrm{C}$ & \multirow{2}{*}{ even } & Cardy/Rome & +++ & $S O$ & 1 \\
& & & ++- & $S O$ & 2 \\
& & & -+0 & $U$ & 3 \\
\hline $\mathrm{T}$ & even & exceptional & +-0 & $S O$ & 6 \\
\hline
\end{tabular}

Note that all allowed continuous Klein bottle amplitudes make their appearance for both odd and even $N$, but in rather different ways. Note also that for even $N$ the diagonal 
invariant (D) allows four different orientifold projections (the case $K_{-+0}$ actually consists of two subcases with opposite signs for all Klein bottle coefficients in the twisted sector), whereas the twist conjugation invariant $(\mathrm{T})$ allows only one. This is strange because we expect these theories to be dual to each other (in the sense of the existence of a one-to-one map between their operators, respecting all correlators). This duality is of course not the T-duality of the circle (which was modded out in the orbifold). We do not know if such a duality has been proved in the literature, but it certainly seems to hold in the simplest case, $N=2$, the tensor product of two Ising models. Note that T-dual circles admit the same number (namely two) of O-plane/D-brane configurations, and the only aspect that differs is the number of allowed D-brane positions. In the orbifold case two probably dual theories have a different number of orientifold projections, corresponding to physically different configurations, with different CP groups. Although this is counterintuitive, on the other hand it does not seem to contradict the duality in an obvious way. Note that also the number of boundary conditions differs for the two mutually dual cases, but this merely corresponds to a different choice of rationally allowed positions for the same D-branes. Note furthermore that for T-dual rational circle theories the number of orientifold choices is the same.

After this enumeration (which is exhaustive for small $N$ ) only four of the twelve potential Klein bottles are realized. Most absences can be explained by a combination of the following facts

- The twist fields $\sigma_{i}, \tau_{i}$ and the fields $\phi_{i}$ must be simultaneously (off)-diagonal in any MIPF. This follows from modular invariance.

- The Klein bottle coefficients of $\phi_{1}$ and $\phi_{2}$ must be identical, because these fields are either each others conjugates, or they fuse to $\partial X \bar{\partial} X$, which must have projection sign 1 . We call these coefficients $K_{\phi}$.

- $K_{\phi}$ can be expressed as $\frac{1}{2}\left(\epsilon_{2}+\epsilon_{1}^{N}\right)$.

- The fusion of $\sigma_{1}$ and $\sigma_{2}$ produces fields $\varphi^{k}$, with $k+N$ odd.

- The fusion of $\sigma_{1}$ and $\tau_{1}$ produces fields $\varphi^{k}$, with $k+N$ even.

The last two points are relevant only if the twist fields appear diagonally. These points imply the following. For conjugation invariants we must have $\epsilon_{3}=0$ and $\epsilon_{2}=-\epsilon_{1}^{N}$. For diagonal invariants we must have $\epsilon_{2}=\epsilon_{1}^{N}$. Furthermore from point 5 we find that $\epsilon_{1}=1$ for $N$ odd. Hence $\epsilon_{2}=1$ for all diagonal invariants. If $\epsilon_{3}=0$ the projections of $\sigma_{1}$ and $\sigma_{2}$ are opposite. This is impossible for $N$ odd, and implies $\epsilon_{1}=-1$ for $N$ even. On the other hand, if $\epsilon_{3} \neq 0$, point 4 implies $\epsilon_{1}=\epsilon_{2}=1$ for $N$ even.

This only leaves one case that was not found, and is also not yet ruled out, namely $\epsilon_{1}=\epsilon_{2}=-1$ for conjugation invariants with even $N$. This case can be ruled out by computing the transverse channel amplitude. It turns out that $\phi_{1}$ and $\phi_{2}$ propagate in the transverse channel, although they are not Ishibashi states. Hence this case must be rejected. 
As an additional check on the result one can now solve the U-NIMrep equations for all other accessible MIPFs as well. The results are in complete agreement with the foregoing: $Z_{D}(p, N)$ has 4 U-NIMreps for $N$ even, 2 for $N$ odd; $Z_{C}(p, N)$ has one U-NIMrep for $N$ even, and 2 for $N$ odd; $Z_{i i}(p, N)$ always has 4 U-NIMreps, whereas $Z_{i j}(p, n), i \neq j$, has none. Finally $Z_{X}(p, N)$ always has 4 NIMreps, except when $N=p^{2}$, in which case it has two. The four U-NIMreps of $Z_{X}$ correspond precisely to two distinct Klein bottle choices for the circle and its dual. For $N=p^{2}$ one ends up in the self-dual point, which explains why one gets only half the number of solutions. In all cases these distinct U-NIMreps correspond to choosing different boundary conjugations for a single NIMrep, although $Z_{C}(3,9)$ and $Z_{D}(3,9)$ have respectively one and two additional NIMreps that do not admit any U-NIMreps, and are presumably spurious. Finally, $Z_{i j}(p, n), i \neq j$ was found to have a single NIMrep which does not admit a U-NIMrep, in agreement with the fact that these MIPFs are asymmetric.

The U-NIMreps for $Z_{C}(p, p q)$ and $Z_{D}(p, p q)$, with $p$ and $q$ prime have been given explicitly in [131], and U-NIMreps for simple current MIPFs are described in [69]. In all other cases these conclusions, as well as the completeness of the entire picture, rely on extrapolation to arbitrary $N$.

\section{Localization and Chan-Paton groups}

In the rational CFT description one can attempt to get information about the boundary and crosscap states by analyzing the Fourier transformation of their coupling to closed string states. This amounts to probing the brane/plane positions by scattering of gravitons [56] (or, equivalently, dilatons or tachyons). This method has a clear physical interpretation in flat space, but becomes less intuitive when applied to compact spaces, although sensible results are obtained for the circle (as explained above) and WZW-models [67] [92]. Apart from the proper physical interpretation, a second caveat is that this method requires a precise knowledge of the boundary and crosscap coefficients. In many cases the latter are obtained by imposing integrality conditions on annulus, Möbius and Klein bottle amplitudes. These amplitudes are not sensitive to sign changes in the coupling to Ishibashi states provided one makes the same sign change in the boundary and the crosscap coefficients. Such sign changes do not affect tadpole cancellation either. In principle the true sign can be determined by solving the sewing constraints, but that has been done only in very few cases. However, in the Cardy case the results of [66] imply that the signs are correct. This should then also apply to all possible choices of orientifold projections, since this is expected to add O-planes in different positions while keeping the branes fixed.

Keeping these caveats in mind, we can compute the positions as follows. The coupling to the fields $\varphi^{k}$ provides a natural set of Fourier components for the couplings. Inspired by the circle results we define a shape function

$$
F(x)=\sum_{k=1}^{N-1}\left(e^{i k x / R}+e^{-i k x / R}\right) C(k)
$$


where $C(k)$ is a boundary or crosscap coefficient ${ }^{2}$ for the coupling to $\varphi^{k}$. This function is periodic with period $2 \pi R$ and symmetric in $x \rightarrow-x$ as well as $\pi R+x \rightarrow \pi R-x$, and therefore it is natural to identify the line segment $[0, \pi R]$ with the orbifold. Note that in the diagonal and conjugation modular invariants of the orbifolds with $R^{2}=\alpha^{\prime} N$ all Ishibashi labels $k$ occur, so that there are no other points with reflection symmetry: the identification of the two orbifold points is unambiguous. By defining $C(-k)=C(k)$ the second term can be used to extent the sum to negative $k$. The coefficients $C(0)-C(\partial X)$ and $C\left(\phi_{1}\right)+C\left(\phi_{2}\right)$ turn out to have precisely the right value to complete the sum to the range $-N \leq k<N$. The resulting function $F(x)$ typically has one or two positive or negative peaks along the orbifold line, which approach $\delta$-functions for large $N$. We interpret the extrema as $\mathrm{O} 0$ plane positions, and the sign as an $\mathrm{O} 0$ charge. For the six cases in the table, the coefficients $C(k)$ for the crosscaps either vanish for all even $k$, or for all odd $k$. The non-vanishing values are all equal to $1 / \sqrt{N}$, up to a sign. If this sign is positive, this leads respectively to opposite charge or same-charge planes at the two endpoints of the orbifold line, $x=0$ and $x=\pi R$. The other possibility we encounter is a $\operatorname{sign}(-1)^{k / 2}$ for even $k$. This shifts the plane positions by $\frac{1}{2} \pi R$, so that they are on top of each other.

Because the orbifold incorporates the circle T-duality, which interchanges D0(O0) with D1(O1) branes (planes) we expect boundary and crosscap states to describe a combination of branes and planes of dimension 0 and 1 . While the D0/O0 positions and charges can be extracted very easily from $F(x)$, this is not the best way to determine the D1/O1 charges. The information is in fact hidden in the linear combinations $C(0)+C(\partial X)$ and $C\left(\phi_{1}\right)-C\left(\phi_{2}\right)$ which are not used in the computation of $F(x)$. A Fourier transform of these two quantities yields identical values on all allowed brane positions in the first case, and alternating values on even and odd positions in the second case. Furthermore in all cases either $C(0)+C(\partial X)$ or $C\left(\phi_{1}\right)-C\left(\phi_{2}\right)$ is zero. Remembering how D1 branes emerged for the rational circle, we are led to the conclusion that a non-vanishing $C(0)+C(\partial X)$ implies the presence of two equal-charge D1/O1 branes/planes, whereas a non-vanishing value of $C\left(\phi_{1}\right)-C\left(\phi_{2}\right)$ implies two opposite-charge D1/O1 branes/planes.

The "charge" referred to above always refers back to the corresponding quantity for the circle, namely the dilaton coupling strength. This allows us to interpret any orbifold brane/plane configuration in terms of a collection of circle configurations of different dimension and charges. Not surprisingly, this interpretation breaks down for branes labelled by twist fields, that have no circle analog. Furthermore the values $C\left(\sigma_{i}\right)$ and $C\left(\tau_{i}\right)$ (which vanish for crosscaps and most boundaries) are also not used, and we do not have a geometric interpretation for these values.

Some more information about brane and plane positions can be gathered from boundary conjugation, which geometrically corresponds to a reflection of a brane through an O-plane. This property affects the Chan-Paton groups of the brane, which is orthogonal

\footnotetext{
${ }^{2}$ We use here the coefficients specified in [69], but without the denominator factors $\sqrt{S_{K i}}$. It is more natural to absorb these factors in the Ishibashi metric for the unoriented annulus, so that the boundary coefficients themselves are independent of the choice of orientifold. See also [67], [92] and [131].
} 
or symplectic for self-conjugate (real) branes and unitary for pairs of conjugate branes. Boundary conjugation and the allowed CP-groups are not affected by the aforementioned sign ambiguities, but the distinction between symplectic and orthogonal for real boundaries does depend on the overall sign of all crosscap coefficients relative to all boundary coefficients. This sign determines the O-plane tension, and whenever we specify a CP group below we have fixed the tension to a negative value, so that the dilaton tadpole can be cancelled (in principle) between D-branes and the O-plane. Orbifold O-planes (unlike circle O-planes) always have non-zero tension.

For the cases discussed listed in the table we find the following positions:

- N even, Diagonal invariant: This is the Cardy case, so boundary labels correspond to primary labels, and the localization analysis should be reliable. The branes with labels 0 and $\partial X$ are at $x=0$, the ones with label $\phi_{i}$ are at $x=\pi R$. The branes with labels $\varphi^{k}$ are localized at points equally spread over the interval. All these branes have in addition a D1 component. The circle-inspired Fourier analysis cannot be trusted for the twisted sector branes and indeed gives contradictory results. The orientifold choices correspond to the four distinct choices of the Klein bottle current, $K=0, \partial X, \phi_{1}$ and $\phi_{2}$. For $K=0$ we get $K_{+++}$, and we find two O0+-planes at the orbifold points plus two $\mathrm{O} 1_{+}$-planes; For $K=\partial X\left(K_{++-}\right)$we get two O0 planes at the orbifold points, and again two $\mathrm{O} 1_{+}$-planes. For $K=\phi_{1}$ or $\phi_{2}\left(K_{-+0}\right)$ we get two coincident $\mathrm{O} 0_{+}$-planes at $x=\frac{1}{2} \pi R$, plus an $\left(\mathrm{O} 1_{+}+\mathrm{O} 1_{-}\right)$configuration. For $K_{+++}$ all CP-groups are $S O$. For $K_{++-}$the CP groups of boundaries $0, \partial X, \phi_{i}, \sigma_{i}$ and $\tau_{i}$ become unitary, while all others remain $S O$. For $K_{-+0}$ all CP groups are unitary, except for $\varphi^{k}, k=N / 2$ and the twist fields with either label 1 or 2 , for which we find orthogonal groups. The group type for the $\varphi^{k}$ branes is easy to understand: if the O-planes are in the middle of the orbifold line segment, they conjugate the branes mutually, except the brane in the center, which is self-conjugate. If the planes are on the endpoints, they conjugate all $\varphi$-branes to their orbifold image, i.e. to themselves, so that they are self-conjugate. A clear geometric picture suggests itself. Given a choice for the orbifold plane, there are two choices for the orientifold plane: on top of it, or orthogonal to it. The first choice leads to $K_{+++}$and $K_{++-}$and mostly self-conjugate branes, the second to $K_{-+0}$ and mostly conjugate brane pairs. The proper geometric interpretation of the CP groups of the eight "special" branes (those not labelled by $\left.\varphi^{k}\right)$ is somewhat less intuitive.

- N even, Twist automorphism: This is an exceptional invariant, and we obtained the boundary and crosscap coefficients numerically for small values of $N$, up to the sign ambiguity described above. Given the Klein bottle amplitude $\left(K_{+-0}\right)$ one can easily compute the crosscap coefficients for all $N: C(0)=C(\partial \phi)=\frac{1}{2}, \quad C\left(\varphi^{2 k+1}\right)=$ $\frac{1}{\sqrt{N}}, C\left(\varphi^{2 k}\right)=0$ This implies an $\mathrm{O}_{+}$plane at $x=0$ and and $\mathrm{O}_{-}$plane at $x=\pi R$. In addition there are two $\mathrm{O} 1_{+}$planes. All $\mathrm{CP}$ groups are orthogonal. We have no explicit formula for the reflection coefficient for arbitrary $N$, although it could be obtained in principle using the methods of [26] applied to the twist orbifold of the 
$c=1$ orbifold (which is the $c=1$ orbifold with four times the value of $N$ ). In the absence of such a formula it is difficult to discuss brane positions with these methods. There is also no canonical labelling of the boundary states.

- N odd, Charge conjugation invariant. The discussion of brane positions is identical to the one for even $N$, except that there is no brane in the middle. There are four possible choices for the Klein bottle current, but $K=\partial X$ and $K=\phi_{2}$ are known to be identical to $K=0, K=\phi_{1}$ respectively, up to interchange of branes [69]. For $K=0\left(K_{+-0}\right)$ the O-plane configuration is as for even $N$, and all CP groups are orthogonal except those of the $\phi_{i}, \sigma_{i}$ and $\tau_{i}$ branes, which are unitary. For $K=1$ $\left(K_{-+0}\right)$ the O-plane configuration is also the same as for even $N$, and all CP-groups are unitary except the ones labelled by $\sigma_{i}, \tau_{i}(i=1$ or 2$)$, which are orthogonal. Apart from the usual eight special branes, these results are analogous to those for even $N$.

- N odd, Diagonal invariant. Here the formulas of [69] apply. The boundary states are labelled by orbits of the simple current $\phi_{1}$. There are $N+1$ branes, two for each label $\varphi^{k}$ with $\mathrm{k}$ even, one labelled by 0 , and one labelled by a twist field. Boundary " 0 " is localized at the orbifold endpoints, the twist field boundary is not localizable, and all other boundaries occupy two symmetric positions on both sides of the center. There are two orientifold projections, distinguished by opposite crosscap signs. One of them yields $K_{+++}$, and all CP-groups are $S O$. The other yields $K_{++-}$, and all groups are $S O$ except the one of the twist field boundary, which is symplectic. The O-plane configuration consists of two $\mathrm{O} 1_{+}$planes, plus two $\mathrm{O} 0_{+}\left(\right.$for $K_{+++}$) and two $\mathrm{O}_{-}$(for $K_{++-}$) planes located at the center. The fact that the boundaries are self-conjugate is understood as a consequence of the fact that each is symmetrically located on each side of the O0-plane. Note however that the picture seems rather different than for even $N$, where the $\mathrm{O} 0$ planes are at the orbifold points. Note also that in this case the caveat regarding signs of the coefficient applies. If we modify all coefficients $C(k)$ by a factor $(-)^{k / 2}$ the O-plane positions are as for even $N$ (however, the brane positions, which also change, are still different than they are for even $N$ ).

Finally we can extract from [131] the crosscap and boundary coefficients for $R^{2}=$ $\alpha^{\prime} p / q, p q$ odd, but only up to signs, as explained above. For the crosscaps the Fourier transformations splits naturally into two sums, one proportional to $1 / \sqrt{q}$ and the other to $1 / \sqrt{p}$. The first gives $\mathrm{O} 0$ planes at multiples of $1 / q$ of the full radius, the second at multiples of $1 / p$, with signs depending on the case considered. These sums are completed by including $C(0)-C(\partial X), C\left(\phi_{1}\right)+C\left(\phi_{2}\right)$ in one of the sums and $C(0)+C(\partial X), C\left(\phi_{1}\right)-C\left(\phi_{2}\right)$ in the other, in agreement with the foregoing discussion. The result can be interpreted either in terms of a circle of radius $R^{2}=\alpha^{\prime} p / q$ or in terms of a circle of radius $R^{2}=\alpha^{\prime} q / p$. The first possibility corresponds a $q$-fold identification of the orbifold line, the second to a $p$-fold identification. In the first case the planes originating from the first Fourier sum are at the endpoints, whereas those from the second one are distributed equally on $p$ points of 
the reduced line segment. It is natural to regard the latter as rational CFT realizations of D1 branes.

For $Z_{C}(p, q)$ two orientifold projections were found in [131], that differ by interchanging $p$ and $q$. The O-plane charges are alternating for one of the Fourier sums, and identical for the other. On the reduced orbifold line segment this can be interpreted as a configuration with two $\mathrm{O} 0_{+}$planes at the end, plus one $\mathrm{O} 1_{+}$and one $\mathrm{O} 1_{-}$plane, and a configuration with one $\mathrm{O} 0_{+}$plane and one $\mathrm{O} 0_{-}$plane at the end, plus two $\mathrm{O} 1_{+}$planes (two, because odd and even points are to be identified with different O1-planes, as in the previous case). For $Z_{D}(p, q)$ there are also two orientifold projections, this time differing by signs in the crosscap coefficients, that flip the two Fourier sums with respect to each other. Using the same interpretation we now get two $\mathrm{O} 0_{+}$planes at the end, combined with either two $\mathrm{O} 1_{+}$ or two $\mathrm{O} 1_{-}$planes. All this is identical to the results for odd, integer radius, except for the positions of the two $\mathrm{O} 0_{+}$planes. But precisely these positions are affected by the unknown signs. This particular kind of simple current MIPF (generated by a $\mathbf{Z}_{4}$-current with fixed points) does not appear in the circle theory and hence the correctness of these signs cannot be tested using brane localization on the circle.

\subsubsection{Orientifolds for arbitrary radius}

In this subsection we argue that the four Klein bottle amplitudes that we obtained in the previous subsection are the only possible ones for arbitrary radius. Thus, we find all possible orientifold maps, that is maps that project out states that are not invariant under the exchange $z \leftrightarrow \bar{z}$. Since the orientifold transformation of $X_{L}$ and $X_{R}$ must square to the identity, the oscillators transform like $a_{n} \rightarrow \bar{a}_{n}$. The only freedom left is in the operators coming from the $z$ independent parts in the expansion of $X_{L}$ and $X_{R}$.

The standard orientifold projection [120] corresponds to the map $X_{L} \rightarrow X_{R}$, giving rise to the amplitude (see (3.10))

$$
K_{+++}=\frac{1}{2} \frac{1}{\eta\left(2 i \tau_{2}\right)} \sum_{k \in \mathbf{Z}} q^{\frac{\alpha \prime}{2}\left(\frac{k}{R}\right)^{2}}+\frac{1}{2} \frac{1}{\eta\left(2 i \tau_{2}\right)} \sum_{m \in \mathbf{Z}} q^{\frac{1}{2 \alpha \prime}(m R)^{2}}+2 \sqrt{\frac{\eta}{\theta_{4}}} .
$$

The first two terms arise from a trace over the states in the untwisted sectors. In the first of these two the orientifold map is inserted, and only the states with zero winding contribute. The second is the one with both the orientifold and orbifold map inserted, and since the KK momentum is not invariant under reflections, only states with no KK momentum contribute. The last term comes from the two twisted sectors; they both contribute in the same amount.

The first variation is to let the operators that create the ground states in the twisted sectors acquire a minus sign under the orientifold transformation. This will result in a minus sign in the last term of (3.10), giving

$$
K_{++-}=\frac{1}{2} \frac{1}{\eta\left(2 i \tau_{2}\right)} \sum_{k \in \mathbf{Z}} q^{\frac{\alpha \prime}{2}\left(\frac{k}{R}\right)^{2}}+\frac{1}{2} \frac{1}{\eta\left(2 i \tau_{2}\right)} \sum_{m \in \mathbf{Z}} q^{\frac{1}{2 \alpha \prime}(m R)^{2}}-2 \sqrt{\frac{\eta}{\theta_{4}}} .
$$


In order to understand which other possible maps are allowed, one has to consider the way the various sectors interact. The untwisted sectors combine according to

$$
(+, n)(+, m)=(+, n+m)
$$

Apart from the standard projection, this equation allows for the map

$$
\begin{gathered}
X_{L} \rightarrow X_{R}+\frac{\pi}{2} \frac{\alpha \prime}{R} \\
X_{R} \rightarrow X_{L}-\frac{\pi}{2} \frac{\alpha \prime}{R},
\end{gathered}
$$

that changes the sign of the states in the sectors with odd winding. Because twisted sectors combine like

$$
(-, \text { odd })(-, \text { even })=(+, \text { odd })
$$

consistency requires that the two twisted sectors contribute with opposite sign after the projection. The resulting amplitude is

$$
K_{+-0}=\frac{1}{2} \frac{1}{\eta\left(2 i \tau_{2}\right)} \sum_{k \in \mathbf{Z}} q^{\frac{\alpha \prime}{2}\left(\frac{k}{R}\right)^{2}}+\frac{1}{2} \frac{1}{\eta\left(2 i \tau_{2}\right)} \sum_{m \in \mathbf{Z}}(-1)^{m} q^{\frac{1}{2 \alpha^{\prime}}(m R)^{2}},
$$

where the contribution from the twisted sectors cancels.

¿From T-duality one can then obtain the last consistent map,

$$
\begin{aligned}
X_{L} & \rightarrow X_{R}+\frac{\pi}{2} R \\
X_{R} & \rightarrow X_{L}+\frac{\pi}{2} R,
\end{aligned}
$$

that changes the sign of states with odd KK momentum. Since $X \rightarrow X+\pi R$, the twisted ground state localized in $X=0$ is swapped for the one localized in $X=\pi R$. This means that 0 and $\pi R$ are no longer fixed points of the orientifold map, whose eigenstates are now localized in $X= \pm \frac{\pi}{2} R$. Moreover, since the trace over the twisted states vanishes after the projection, there is no contribution from the twisted sectors to the Klein bottle amplitude, whose form is

$$
K_{-+0}=\frac{1}{2} \frac{1}{\eta\left(2 i \tau_{2}\right)} \sum_{k \in \mathbf{Z}}(-1)^{k} q^{\frac{\alpha \prime}{2}\left(\frac{k}{R}\right)^{2}}+\frac{1}{2} \frac{1}{\eta\left(2 i \tau_{2}\right)} \sum_{m \in \mathbf{Z}} q^{\frac{1}{2 \alpha \prime}(m R)^{2}} .
$$

In order to understand why the choice $\epsilon_{1}=\epsilon_{2}=-1$ in eq. (3.10) is not allowed, we have to analyze the transverse channel. We only need to consider the untwisted sector, so we concentrate on the case $K_{--0}$. The Klein bottle in the direct channel depends on $2 i \tau_{2}$, the modulus of the doubly-covering torus. The Klein bottle in the transverse channel is obtained performing an $S$ modular transformation on the modulus of the doubly-covering torus, that is writing the amplitude in terms of $\ell=1 / 2 \tau_{2}$, the proper time in the transverse 
channel, describing a closed string propagating between two orientifold planes. The endresult of this transformation (see for instance [8] for a review) is

$$
\begin{aligned}
& \tilde{K}_{++ \pm}=\frac{1}{2} \frac{1}{\eta(i \ell)} R \sqrt{\frac{2}{\alpha \prime}} \sum_{k \in \mathbf{Z}} q^{\frac{1}{\alpha^{\prime}}(k R)^{2}}+\frac{1}{2} \frac{1}{\eta(i \ell)} \frac{1}{R} \sqrt{2 \alpha \prime} \sum_{m \in \mathbf{Z}} q^{\alpha^{\prime}\left(\frac{m}{R}\right)^{2}} \pm 2 \sqrt{\frac{\eta}{\theta_{2}}} \\
& \tilde{K}_{+-0}=\frac{1}{2} \frac{1}{\eta(i \ell)} R \sqrt{\frac{2}{\alpha \prime}} \sum_{k \in \mathbf{Z}} q^{\frac{1}{\alpha^{\prime}}(k R)^{2}}+\frac{1}{2} \frac{1}{\eta(i \ell)} \frac{1}{R} \sqrt{2 \alpha \prime} \sum_{m \in \mathbf{Z}} q^{\alpha^{\prime}\left(\frac{m+1 / 2}{R}\right)^{2}} \\
& \tilde{K}_{-+0}=\frac{1}{2} \frac{1}{\eta(i \ell)} R \sqrt{\frac{2}{\alpha \prime}} \sum_{k \in \mathbf{Z}} q^{\frac{1}{\alpha^{\prime}}(k+1 / 2)^{2} R^{2}}+\frac{1}{2} \frac{1}{\eta(i \ell)} \frac{1}{R} \sqrt{2 \alpha \prime} \sum_{m \in \mathbf{Z}} q^{\alpha^{\prime}\left(\frac{m}{R}\right)^{2}} \\
& \tilde{K}_{--0}=\frac{1}{2} \frac{1}{\eta(i \ell)} R \sqrt{\frac{2}{\alpha \prime}} \sum_{k \in \mathbf{Z}} q^{\frac{1}{\alpha^{\prime}}(k+1 / 2)^{2} R^{2}}+\frac{1}{2} \frac{1}{\eta(i \ell)} \frac{1}{R} \sqrt{2 \alpha \prime} \sum_{m \in \mathbf{Z}} q^{\alpha^{\prime}\left(\frac{m+1 / 2}{R}\right)^{2}},
\end{aligned}
$$

where here $q=e^{-2 \pi \ell}$. The states that contribute to the Klein bottle amplitude in the transverse channel are closed-string states propagating between two orientifold planes. In $\tilde{K}_{++ \pm}$only states with even KK momentum or even winding contribute in the untwisted sector. In $\tilde{K}_{+-0}$ only states with odd KK momentum or even winding contribute. This is consistent with the direct channel, since $K_{+-0}$ projects out only states with odd winding. The same is valid for $\tilde{K}_{-+0}$, where only states with even KK momentum and odd winding contribute, while in the direct channel the states with odd KK momentum are projected out. Finally, in the case of $\tilde{K}_{--0}$ states with odd KK momentum and states with odd winding contribute to the transverse amplitude, but these states are both projected out by $K_{--0}$, so that this Klein bottle projection is not consistent.

¿From the transverse channel analysis one can also derive the position of the orientifold planes for the various Klein bottles. In all cases, namely the standard orientifold projection $X_{L} \rightarrow X_{R}$, and the ones given in (3.15) and (3.18), the map has no fixed points, and this corresponds to introducing O1-planes. Once the orbifold map $X \rightarrow-X$ is implemented, all these maps develop fixed points, where O0-planes are located. This means that all these amplitudes describe a configuration of two O1 planes, and two O0 planes located at the fixed points of the 'orientifold+orbifold' $(\Omega r)$ map. The twisted sector corresponds in the transverse channel to closed string states propagating between an O1 plane and an O0 plane.

In the case of $K_{++ \pm}$, the $\Omega$ and $\Omega r$ maps are respectively $X \rightarrow X$ and $X \rightarrow-X$, and the two O1 planes have the same tension, as well as the two O0 planes. The two sign options for the twisted sector correspond to the fact that the tension of the O0 planes can be positive or negative with respect to the tension of the O1 planes ${ }^{3}$. Since the $\Omega r$ map has fixed points in 0 and $\pi R$, the position of the two O0-planes coincides with the two fixed points of the orbifold. Applying the T-duality transformation $X=X_{L}+X_{R} \rightarrow X^{\prime}=X_{L}-X_{R}$, and $R \rightarrow R^{\prime}=\alpha \prime / R$, one can see that these two Klein bottles are both self-T-dual. In

\footnotetext{
${ }^{3}$ The $K_{++-}$case is analogous to the six-dimensional brane supersymmetry breaking model of [10], in which the O5-planes and the O9-planes have opposite tension.
} 
the case of $K_{+-0}$, the $\Omega$ projection is given in (3.15), and it maps $X$ to itself, meaning that the two $\mathrm{O} 1$ planes have the same tension. The $\Omega r$ projection maps $X$ to $-X$, so that the O0-planes are located at the fixed points of the orbifold. In order to determine the tension of the O0-planes, one has to consider how the $\Omega r$ transformation acts on the T-dual coordinate. In this case one has $X^{\prime} \rightarrow X^{\prime}+\pi R^{\prime}$, and the shift in the dual coordinate is a manifestation of the fact that the two O0-planes have opposite tension. The Klein bottle amplitude corresponds thus to the configuration $\mathrm{O} 1_{+} \oplus \mathrm{O} 1_{+} \oplus \mathrm{O} 0_{+} \oplus \mathrm{O} 0_{-}$, and the twisted sector cancels because of the opposite contribution from the two orbifold fixed points, where the O0-planes are located. Finally, in the case of $K_{-+0}$, the $\Omega$ projection is given in (3.18), and it maps $X$ to $X+\pi R$, so that the two O1-planes have opposite tension, while $\Omega r$ maps $X$ to $-X=\pi R$, so that the O0-planes are located in the middle of the orbifold segment. In this case the twisted sector cancels separately in any of the two (coincident) orientifold fixed points. T-duality maps this configuration to the previous one.

In all these cases the locations and charges of the O0 planes and the charges of the O1 planes agree with the results obtained from the CFT analysis in subsection 3.3.1, except those for the $K_{++ \pm}$Klein bottle of the D-invariants for odd $N$. In that case the charges are the same, but the two $\mathrm{O}_{+}$planes were found in the center rather than at the edges. But this was precisely a non-Cardy case, where the signs of the crosscap coefficients (crucial for the precise location) are not determined.

The foregoing discussion was for arbitrary radius, and seemed to rely in all cases on the standard orbifold map $X \rightarrow-X$. At rational radii the various orientifolds occur in combinations with specific partition functions, which require different orbifold maps, discussed in the appendix. This changes the map $r$ in the foregoing discussion, and hence its fixed points. However, it also changes $\Omega r$, whose fixed points determine the O-planes. It is easy to see that both modifications cancel, so that the relative position of orbifold fixed points and O0-plane positions remains unchanged. Note that nothing in the analysis in this section imposed any relation between the orientifold map and the orbifold map in rational points. This relation was found in subsection 3.3.1 and makes use of OPEs involving distinct twist fields. We did not consider twist fields in this section, and furthermore for non-rational radius they cannot be distinguished, hence there is no reason to expect such a relation to emerge.

\subsubsection{Orientifolds of exceptional MIPFs}

As mentioned in section 3.3.1, the orbifold has exceptional MIPFs, constructed using an automorphism, $\omega$, which leaves the fusion coefficients invariant: $N_{i j}{ }^{k}=N_{\omega(i) \omega(j)}{ }^{\omega(k)}$. The exceptional torus partition functions obtained from the chiral algebra of the CFT at square radius $R^{2}=\alpha \prime p q$, with $p$ and $q$ odd prime numbers, are

$$
T=\sum \chi_{i} \delta_{i \omega(j)} \bar{\chi}_{j}, \quad T=\sum \chi_{i} C_{i \omega(j)} \bar{\chi}_{j} .
$$

In [131], these two invariants were called "diagonal + automorphism" (D+A) and "Cardy + automorphism" $(\mathrm{C}+\mathrm{A})$ respectively. Geometrically, these two tori describe a free boson 
compactified on an orbifold of radius $R^{2}=\alpha \prime p / q$ and its T-dual.

We first review the results of [131] about orientifold projections. In the D+A case, the trivial Klein bottle, that is $K_{i}=1$ for all the fields that couple diagonally on the torus, is allowed. A second Klein bottle is also allowed, with $K_{i}=-1$ for the twist fields and $K_{i}=1$ for the other diagonal fields. In the $\mathrm{C}+\mathrm{A}$ case, surprisingly the trivial choice $K_{i}=1$ for all the fields coupling diagonally on the torus is not allowed. There are two Klein bottles. One has $K_{\phi_{k}}=-1$ when $k$ is an odd multiple of $p$ and $K_{i}=1$ otherwise, and the other is obtained exchanging $p$ with $q$.

Looking at the resulting amplitudes as functions of the orbifold radius, one realized that these Klein bottles are precisely the ones obtained in section 3.3.2, for a bosonic string compactified on a circle of square radius $R^{2}=\alpha \prime p / q$. In particular, the Klein bottles of the $\mathrm{D}+\mathrm{A}$ modular invariant are $K_{+++}$and $K_{++-}$, while the Klein bottles of the $\mathrm{C}+\mathrm{A}$ modular invariant are $K_{+-0}$ and $K_{-+0}$. Since the twisted sector is not diagonal for the $\mathrm{C}$ modular invariant, this is the only possibility that is allowed in light of the results of the previous subsection, and thus the results of [131] are completely consistent with the orientifold projections that are allowed for arbitrary radius.

\subsection{Conclusions}

We have identified four distinct orientifold projections for the $c=1$ orbifolds. Geometrically, they can be described most easily starting from the O0 plane configurations of the T-dual circle. The $\left(\mathrm{O}_{+}, \mathrm{O}_{-}\right)$configuration has only one axis of symmetry, namely the line through the O0-planes. Hence the orbifold and O-plane directions must line up, and the orbifold $\mathrm{O} 0$ planes are at its endpoints. The configuration $\left(\mathrm{O}_{+}, \mathrm{O}_{+}\right)$has two axes of symmetry, and the orbifold reflection line is either on top of or orthogonal to the orientifold line. Then the O0-planes are respectively at the endpoints or on top of each other in the center.

In the circle theory on can distinguish two T-dual orientifold maps, one of the form $X_{L} \rightarrow+X_{R}+$ const and one of the form $X_{L} \rightarrow-X_{R}+$ const. The former has fixed points in $X_{L}-X_{R}$, but not in $X_{L}+X_{R}$, whereas for the latter it is just the other way around. Therefore the former gives rise to O1 planes on the circle and the latter to O0-planes on the T-dual circle. The orbifold map (which has fixed points both in $X_{L}-X_{R}$ and $X_{L}+X_{R}$ ) transforms the two types of orientifold maps into each other, so that both $\mathrm{O} 1$ and $\mathrm{O} 0$ planes are present. Inspection of the transverse channel show that the charges of the O1 planes are identical if the orbifold fixed plane and the orientifold plane coincide, whereas they are opposite if these fixed planes are orthogonal. Allowing for an additional relative sign between the $\mathrm{O} 1$ and $\mathrm{O} 0$ planes then gives a total of four configurations (since the overall sign is irrelevant $):\left(\mathrm{O} 1_{+} \oplus \mathrm{O} 1_{+}\right) \oplus\left(\mathrm{O} 0_{+} \oplus \mathrm{O} 0_{+}\right),\left(\mathrm{O} 1_{+} \oplus \mathrm{O} 1_{+}\right) \oplus\left(\mathrm{O} 0_{-} \oplus \mathrm{O} 0_{-}\right)$, $\left(\mathrm{O} 1_{+} \oplus \mathrm{O} 1_{+}\right) \oplus\left(\mathrm{O} 0_{+} \oplus \mathrm{O} 0_{-}\right)$and $\left(\mathrm{O} 1_{+} \oplus \mathrm{O} 1_{-}\right) \oplus\left(\mathrm{O} 0_{+} \oplus \mathrm{O} 0_{+}\right)$. This argument also shows why a fifth logical possibility, $\left(\mathrm{O} 1_{+} \oplus \mathrm{O} 1_{-}\right) \oplus\left(\mathrm{O} 0_{+} \oplus \mathrm{O} 0_{-}\right)$, cannot occur.

This intuitive argument was worked out in detail in section 3.3.2, and is backed up by the complete solution for U-NIMreps for rational CFT. The latter classification can be 
done exhaustively, but this is necessarily limited to a few rational points. We have shown that all four orientifolds are realized in all rational points, although in rather different ways. We have also shown how a known, but initially surprising solution at $R^{2}=\alpha^{\prime} p / q$ fits in perfectly with the continuum.

At arbitrary $R$ we cannot rigorously rule out additional solutions, but in view of the agreement between the continuous $R$ and the rational CFT descriptions, any deviations would be quite surprising.

A few open problems remain. While all methods agree on the O-plane charges, there is a discrepancy on their precise positions in one case, interestingly precisely the case were the CFT results are least reliable. Secondly, the nature of the duality between diagonal and conjugation invariants of the rational orbifolds needs to be clarified. Finally, in the geometric description, applied to rational radii, the link between the choice among those two invariants and the orientifold map is not manifest.

\section{Acknowledgments}

We would like to thank Christoph Schweigert and Yassen Stanev for discussions and Herman Verlinde for clarifying some points in [57]. A.N.S. wishes to thank IMAFF-CSIC, Madrid, where part of this work was done, for hospitality. The work of F.R. and A.N.S. has been performed as part of the program FP 52 of the Foundation for Fundamental Research of Matter (FOM), and the work of T.P.T.D. and A.N.S. has been performed as part of the program FP 57 of FOM. The work of B.G.-R. and A.N.S. has been partially supported by funding of the Spanish "Ministerio de Ciencia y Tecnología", Project BFM2002-03610. 


\section{Appendix: Orbifold maps}

Here we will discuss how the various partition functions enumerated in section 3.3.1 can be obtained from the circle theory. The standard description of orbifolds starts with a circle theory, from which the $Z_{2}$-symmetry $X \rightarrow-X$ is modded out. It is not hard to see that applying this map to the circle theories $Z(p, N)$ or $Z(N / p, N)$ (with $1 \leq p \leq \sqrt{N}$ one obtains in both cases the orbifold theory $Z_{\ldots}(p, N)$. The problem is that in the rational case the orbifold partition function has an additional label D, C or $i j$. Since the distinction is not made by the T-duality of the circle, there must be more than one way to do the orbifold map. Obviously one can generalize it to $X \rightarrow a-X$, i.e. rotating the plane of reflection, but this does not have the desired effect.

It turns out that one must consider the chiral orbifold map

$$
X_{L} \rightarrow a_{L}-X_{L} ; \quad X_{R} \rightarrow a_{R}-X_{R}
$$

On the vertex operators $V(k)$ corresponding to the fields $\varphi^{k}$ the only effect is a phase between the two terms of which they consist; but the effect is more important for the generators of the chiral algebra and the fields $\phi_{1}$ and $\phi_{2}$, which make the difference between the rational and the non-rational case (we will ignore the twist fields here, since the difference between the various partition functions is already clear in the untwisted sector). Note that the circle theory operators from which $\phi_{1}$ and $\phi_{2}$ originate, which have $k= \pm N$ and chiral ground state multiplicity 2, appear in an identical way for a circle and its T-dual.

The dependence of these vertex operators on $a_{L}$ and $a_{R}$ is as follows for the chiral algebra generators

$$
W_{L}=V(R, 0)+e^{i a_{L} \frac{2 R}{\alpha^{\prime}}} V(-R, 0)
$$

and

$$
W_{R}=V(0, R)+e^{i a_{R} \frac{2 R}{\alpha^{\prime}}} V(0,-R)
$$

where

$$
V(r, s)=e^{i \frac{2 r}{\alpha^{\prime}} X_{L}} e^{i \frac{2 s}{\alpha^{\prime}} X_{R}}
$$

For the other four circle operators with $|r|=|s|=\frac{1}{2} R$ we get two invariant combinations

$$
V^{A}=V(+,+)+e^{i \frac{R}{\alpha^{\prime}}\left(a_{L}+a_{R}\right)} V(-,-)
$$

and

$$
V^{B}=V(+,-)+e^{i \frac{R}{\alpha^{\prime}}\left(a_{L}-a_{R}\right)} V(-,+),
$$

with the arguments " + " and "-" denoting $+R / 2$ and $-R / 2$ respectively.

The operators $V^{A}$ and $V^{B}$ are Virasoro-degenerate, but are distinguished by the chiral algebra operators $W_{L}$ and $W_{R}$. In order to relate these operators to a partition function interpretation we need to combine $V^{A}$ and $V^{B}$ into chiral algebra eigenstates. For this we need the OPE of these operators, and here an important rôle is played by the cocycle 
factors that should be added to these operators [100]. In this case these cocycles can be conveniently represented by Pauli matrices $\left(\sigma_{3}\right)^{m}\left(\sigma_{1}\right)^{n}$ where $m$ and $n$ are the winding and momentum quantum numbers of the operator. It is easy to see that $W_{L}$ and $W_{R}$ acquire a factor $\sigma_{3}\left(\sigma_{1}\right)^{N}, V(+,+)$ and $V(-,-)$ a factor $\left(\sigma_{1}\right)^{N}$ and $V(+,-)$ and $V(-,+)$ a factor $\sigma_{3}$. Hence for even $N$ the cocycles do not change anything in comparison with the "naive" OPE. For arbitrary $N$ the chiral algebra eigenstates are found to be

$$
V(+,+)+e^{i \frac{R}{\alpha^{\prime}}\left(a_{L}+a_{R}\right)} V(-,-) \pm\left(i^{N} e^{i \frac{R}{\alpha^{\prime}} a_{R}} V(+,-)+i^{N} e^{i \frac{R}{\alpha^{\prime}} a_{L}} V(-,+)\right) .
$$

For even $N$ this can be factorized as

$$
\left[V_{L}(+) \pm e^{i \frac{R}{\alpha^{\prime}} a_{L}} V_{L}(-)\right]\left[V_{R}(+) \pm e^{i \frac{R}{\alpha^{\prime}} a_{R}} V_{R}(-)\right]
$$

with correlated signs in the two factors; for odd $N$ it cannot be factorized. For these operators to have sensible reality properties, $a_{L}$ and $a_{R}$ must be quantized as multiples of $\alpha^{\prime} \pi / R$, the allowed positions in the rational CFT description (these are precisely the allowed brane positions on the circle; any other value would not allow a rational CFT interpretation). Then one finds that for $N$ even the operators are real, and for $N$ odd they are each others conjugate, in agreement with the modular matrix $S$ [57].

For the standard case $a_{L}=a_{R}=0$, and for $N$ even, the operators have the expected "cos cos" and "sin sin" form that is indicative of the diagonal invariant. By choosing $a_{L}=0$, $a_{R}=\alpha^{\prime} \pi / R$ one can change this to a "cos sin" and "sin cos" form, corresponding to the conjugation invariant. For odd $N$ the results are similar. The operators for $a_{L}=a_{R}=0$ can be written in the form "cos cos $\pm i$ sin sin" and they change to "cos $\sin \pm i \sin \operatorname{cos"~for~}$ $a_{L}=0, a_{R}=\alpha^{\prime} \pi / R$. These two cases should correspond, respectively, to the diagonal and charge conjugation invariant of the odd $N$ orbifold. To get the heterotic orbifold invariants we may choose $a_{L}=0, a_{R}=\alpha^{\prime} \pi / 2 R$. Note that this value for $a_{R}$ does not belong to the set of allowed positions, but it is an allowed position for the orbifold with twice the value of $R$. The heterotic theory is obtained as a chiral algebra extension of the latter CFT. The term in the partition function corresponding to $V^{A}$ and $V^{B}$ has multiplicity 2, and it is a simple current fixed point, which cannot be resolved using the orbifold data alone. Hence the reality properties of these operators are not determined.

The cocycle factors are also needed in the operators $\Omega$ that implement the various orientifold maps on the vertex operators. In some cases one has to include a factor $\sigma_{3}$ in these operators, which affects the result only for odd $N$ and only when acting the operators $V^{A}$ and $V^{B}$. 
On Orientifolds of $\mathrm{c}=1$ Orbifolds 


\section{Chapter 4}

\section{Supersymmetric Standard Model Spectra from RCFT orientifolds}

\subsection{Introduction}

String theory is hoped to be a consistent theory of quantum gravity, with the special feature that it strongly constrains the matter it can couple to. Although direct experimental tests of new predictions seem out of reach for the moment, it can at least be tested theoretically by verifying its internal consistency, in particular with regard to gravity, and by checking that the limited set of matter it can couple to includes the standard model. We may be lucky enough that the way the standard model is embedded in string theory implies predictions for future experiments, but it may also happen that using all known experimental constraints we are still left with more than one, or even a huge number of possibilities. But at present it is still a serious challenge to find even one "string vacuum" (which may actually be a metastable, approximate ground state) that is a credible standard model candidate. To find such a vacuum requires an in-depth analysis of known candidates based on robust criteria derived from experiment. However, even within the known classes of vacua, large areas have remained unexplored so far. As longs as that is the case, nature would have to be very kind to us to allow us to find the ground state on which our universe is based. In this paper we want to make a modest step towards broadening the set of accessible vacua, by means of a systematic exploration of orientifolds of Gepner models. It turns out that this class is very rich, and includes an abundance of standard model-like spectra far beyond anything that has come out of string theory so far. Some early, and partial results were reported in [59].

For a variety of reasons we will require the string spectrum to be supersymmetric. The first reason is phenomenological. Although we do not commit ourselves to a supersymmetry breaking mechanism or scale, the most obvious scenario is the standard one: supersymmetry breaking at a few $\mathrm{TeV}$, induced by gaugino condensation in a hidden sector (which exists in most of our models), with supersymmetry playing the role of protecting the gauge hierarchy. Indeed, such a hierarchy inevitably exist in these models, since six dimensions 
are compactified on a Calabi-Yau manifold at a rational point in moduli space, and hence there is no reason to expect some of the compactified dimensions to be extremely large. There are two other reasons why we want to keep supersymmetry unbroken. First of all, we can then be certain that the four-dimensional strings we construct are stable and consistent. But the most important reason is a practical one. Space-time supersymmetry has the effect of extending the world-sheet chiral algebra, thereby organizing the fields into a smaller number of primaries. This is what makes our computations manageable in practice.

The use of rational conformal field theory (RCFT) in these constructions has wellknown advantages and disadvantages. The advantage of the algebraic approach is that we can explore a large class of models with uniform methods. But clearly the disadvantage is that one ends up in a special point in moduli space, both with regard to the Calabi-Yau manifold, as well as the choice of branes wrapping it. It is not reasonable to expect such a ground state to be exactly the standard model, because many observable quantities, such as quark and lepton masses and gauge couplings will depend on the moduli, which have been fixed at a specific value. Clearly we should focus on those features that do not depend on the moduli. The primary feature to consider is then of course the chiral spectrum.

Apart from supersymmetry breaking there are several other important issues that we do not consider here, such as standard model symmetry breaking, Yukawa couplings, Majorana masses for the neutrinos, etc. We see our results therefore mainly as a first exploration of some interesting regions in the huge landscape of possible models. Once a promising type of model has been identified, one may try to explore it in more detail, either by CFT perturbations in the neighborhood of the special point, or by constructing the corresponding Calabi-Yau and the set of branes on it, using the RCFT results as a guideline. Even within the context of RCFT one could push these models further and compute certain couplings, but unfortunately the required CFT techniques are not yet available for all couplings. For example, three-point couplings between open strings are in principle computable in RCFT, but to develop this formalism to include non-trivial modular invariant partition functions and simple current fixed points would still require a substantial amount of work. Gauge couplings, on the other hand, are easily computable, and we do so in all cases.

Since we end up with a very large set of solutions, our results should give a reasonable idea of what kind of spectra one may expect, and one can perform some statistical analysis on this set, somewhat similar in spirit (though with a quite different philosophy) to the approach presented in [62]. In addition, despite the inherent limitations of the algebraic approach, one may explore the effect of brane moduli as well as some Calabi-Yau moduli. The former, since for a given CY-manifold we typically find a large number of spectra, which can be interpreted in terms of branes in different discrete positions; the latter, because some distinct Gepner points may lie on the same Calabi-Yau moduli space. Especially the brane position moduli seem to be probed rather effectively by rational points, in certain cases.

\subsubsection{Brane configurations considered}

In this paper we consider boundary states in a rational type IIB CFT. The relevant physical open string quantities are annulus and Moebius coefficients. For the sake of clarity, it is 
convenient to present the models in terms of an intersecting brane picture, although such a picture is not really used in our construction. This picture would be appropriate in a large volume limit and for type IIA string, the mirror of what we consider here. The geometric interpretation of the construction considered here is presumably in terms of magnetized D3 and D7 branes [30]. In the following, by the "intersection" of two branes a and b we mean $\left.\sum_{i} A_{a b}^{i} \chi^{i}(\tau / 2)\right|_{0}$, where $A_{a b}^{i}$ is an annulus coefficient and $\chi$ is the character restricted to massless states. By the "chiral intersection" we mean the same quantity restricted to chiral states.

We consider here a specific type of intersecting brane models, based on a four-stack configuration with a baryon brane, a weak brane (or left brane), a right brane and a lepton brane, labeled a,b,c,d respectively [98]. These are the minimal brane configurations with baryon and lepton number conservation and all quarks and leptons realized as bifundamentals. The Chan-Paton gauge groups associated with these branes contain the standard model gauge group. In addition we allow "hidden branes", with gauge groups with respect to which all standard model particles are neutral. These branes were introduced to cancel massless tadpoles, but their gauge groups may play a useful phenomenological rôle, in particular for gaugino condensation.

The a and d branes are required to be complex, and carry Chan-Paton group $U(3)_{\mathrm{a}}$ and $U(1)_{\mathrm{d}}$ respectively. The former contains the standard model gauge group $S U(3)$. The overall phase factor of $U(3)_{\mathrm{a}}$ corresponds to baryon number, and the $U(1)_{\mathrm{d}}$ to lepton number. In the standard model these symmetries are not gauged, and anomalous. In string theory these anomalies are canceled by Green-Schwarz terms, involving a bilinear coupling of the "bary-photon" and "lepto-photon" to massless two-form fields. These couplings produce a mass for the linear combination $B+L$ of these $U(1)$ bosons, breaking the corresponding combination of baryon and lepton number. Nevertheless the broken symmetry still prevents the appearance of dangerous baryon and lepton number violating couplings at least perturbatively [98].

The $\mathrm{b}$ and $\mathrm{c}$ branes may be real or complex. In the standard four-stack realization of the standard model the first family emerges as follows if they are both complex, with CP-groups $U(2)_{\mathrm{b}}$ and $U(1)_{\mathrm{c}}$ respectively

$$
\begin{aligned}
(u, d) & :[\mathrm{a}, \mathrm{b}] \text { or }[\mathrm{a}, \mathrm{b} *] \\
u^{c} & :\left[\mathrm{a}^{*}, \mathrm{c}\right] \\
d^{c} & :\left[\mathrm{a}^{*}, \mathrm{c}^{*}\right] \\
\left(e^{-}, \nu\right) & :[\mathrm{b}, \mathrm{d}] \text { or }\left[\mathrm{b}^{*}, \mathrm{~d}\right] \\
e^{+} & :\left[\mathrm{c}, \mathrm{d}^{*}\right] \\
\nu^{c} & :\left[\mathrm{c}^{*}, \mathrm{~d}^{*}\right]
\end{aligned}
$$

Here $[x, y]$ denote strings beginning on brane $x$ and ending on brane $y$, and $x^{*}$ is the brane conjugate to $x$. The $Y$-charge of the standard model is given by the linear combination

$$
Y=\frac{1}{6} Q_{\mathrm{a}}-\frac{1}{2} Q_{\mathrm{c}}-\frac{1}{2} Q_{\mathrm{d}}
$$


The overall phase symmetry in $U(2)_{\mathrm{b}}$ is always anomalous with respect to the $a$ and the $d$ branes and the corresponding gauge boson acquires a mass; the surviving gauge group is $S U(2)_{W}$. Note that $B+L$ and $U(2)_{\mathrm{b}}$ have independent anomalies with respect to the standard model, so that there is no linear combination of these phases symmetries that remains unbroken.

The standard model weak gauge group can also be constructed out of real branes on top of an orientifold plane, yielding $S p(2)$. Since the spectrum is real with respect to the c-brane, we may allow the group $O(2)_{\text {c }}$ here instead of $U(1)_{\mathrm{c}}$, with $Q_{\mathrm{c}}$ replaced by the (properly normalized) $O(2)$ generator in the definition of the charge. Since $O(2)$ branes differ from $S p(2)$ branes only by a Moebius sign, we decided to allow the latter as well. Strictly speaking this is a departure from our philosophy of looking only for the simplest standard model realizations, but these models are as easy to look for as $O(2)$ models, and have the interesting feature of yielding "left-right symmetric" models with an $S U(2)_{L} \times S U(2)_{R}$ gauge group. Such gauge groups appear as part of the symmetrybreaking chain of the Pati-Salam model, and indeed in some examples there are related spectra with the $d$ brane on top of the $a$ brane, yielding precisely the Pati-Salam model. Then we end up with following six types of models:

$$
\begin{array}{ll}
\text { Type } 0 & U(3) \times S p(2) \times U(1) \times U(1) \\
\text { Type } 1 & U(3) \times U(2) \times U(1) \times U(1) \\
\text { Type 2 } & U(3) \times S p(2) \times O(2) \times U(1) \\
\text { Type } 3 & U(3) \times U(2) \times O(2) \times U(1) \\
\text { Type } 4 & U(3) \times S p(2) \times S p(2) \times U(1) \\
\text { Type 5 } & U(3) \times U(2) \times S p(2) \times U(1)
\end{array}
$$

The complete spectrum of these theories can contain massless vector bosons from three sources: the standard model part of the open sector, as listed above, additional "hidden" branes, and the closed sector. The latter gauge bosons are nearly inevitable, but do not have minimal couplings to the quarks and leptons. The gauge bosons from the hidden sector may be absent altogether, but in any case do not couple to the standard model particles. If we ignore these two kinds of vector bosons, the gauge group is quite close to that of the standard model. As a Lie-algebra, it is a non-abelian extension of the standard model only for types 4 and 5 ; in all other cases we get the standard model with at most one additional non-anomalous $U(1)$ factor, $B-L$.

The gauge bosons coupling to the non-anomalous symmetries $Y$ and $B-L$ can acquire a mass from Green-Schwarz type two-point couplings to two-form fields, provided that these couplings do not generate a contribution to the anomaly. We find that in most cases such a mass contribution is absent, and that $Y$ is more likely to acquire a mass than $B-L$. The latter statement is based only on the models presented in [59], where masslessness of $Y$ was only used as an a posteriori check. In the present paper brane stacks yielding non-zero $Y$-mass were eliminated before attempting to solve the tadpole equations, but no 
condition was imposed on the $B-L$ mass. We found a massive $B-L$ photon in about $3 \%$ of the type- 0 models, and for none of the type- 1 models (of which we found only very few). There are even models with a massive $B-L$ and no extra branes at all. These models (22in total) have precisely the standard model gauge group from the open sector, but there are still 18 additional Ramond-Ramond vector bosons from the closed sector.

We expect masslessness of the Y-boson, in addition to lepton and quark chirality and supersymmetry, to be among the features of these models that are unaffected by generic perturbations around the rational point. The potential origin of a Y-boson mass would be the generation of a two-point coupling to one of the RR two-forms away from the rational point. However, such a two-point coupling would very likely generate an anomalous contribution in combination with a three-point coupling of the same RR-two form to two gauge bosons.

The same logic applies to the $B-L$ gauge boson. If it is massless at the string level, it should acquire a mass trough a fundamental or dynamical Higgs mechanism, just as the $Z$ and $W$ bosons. Candidates for the required Higgses are the sneutrinos or two standard model/ hidden sector bifundamentals (one ending on the c-brane and one on the d-brane), bound by a hidden sector gauge group.

Most other features cannot be expected to survive generic perturbations. In particular this concerns the massless particles that are non-chiral with respect to $G_{\mathrm{S}}$, namely the mirrors, rank-2 tensors, leptoquarks and standard model/hidden sector bifundamentals. It seems plausible that for many of them masslessness is an artifact of being in a rational point in moduli space. They will get a mass when the moduli are changed, and one can investigate this by computing the coupling of the closed string moduli to the massless fermions. However, some of the massless particles correspond to brane position moduli, and hence to flat directions in the superpotential. One of the problems we have in investigating this and other more detailed phenomenological issues is that we have to find a way to do a meaningful analysis for a huge number of solutions.

There are many other brane configurations that would yield standard-model-like spectra. For example another attractive possibility might be to start with a $U(5)$ stack (realizing an $S U(5)$ GUT) plus other branes or the reduction of such a stack to $U(3) \times U(2)$. In such models some quarks and leptons emerge as anti-symmetric tensors and baryon and lepton number are not symmetries. Models of this type were studied in [48], but so far with the disappointing result that there are additional chiral symmetric tensors of $S U(5)$. In principle one could search for models of this kind in exactly the same way, just as one could search for Pati-Salam type models. It goes without saying that if we were to relax some constraints and allow for example chiral exotics or diagonal embeddings of standard model factors in the CP group, then the number of solution would almost certainly explode. Nevertheless such options, although unattractive, are not necessarily ruled out experimentally. 


\subsubsection{Chirality}

Let us explain more precisely what we mean by "getting the standard model from string theory", since this issue tends to cause confusion. Quite generally, one might accept any string spectrum if the gauge group $G_{\mathrm{S}}$ emerging directly from open or closed strings contains $G_{\mathrm{SM}}=S U(3) \times S U(2) \times U(1)$, and if the fermion representation of $G_{\mathrm{S}}$ reduces to three times $\left(3,2, \frac{1}{6}\right)+\left(3^{*}, 1, \frac{1}{3}\right)+\left(3^{*}, 1,-\frac{2}{3}\right)+\left(1,2,-\frac{1}{2}\right)+(1,1,1)$, written in terms of left-handed Weyl fermions. These should be the only fermions that are chiral with respect $S U(3) \times S U(2) \times U(1)$. If at this stage there were additional chiral particles one could still imagine mechanisms that give them a sufficiently large mass after standard model symmetry breaking and $S U(3)$ confinement, but if that were true we simply need more experimental input to go ahead. There may be additional massless fermions that are nonchiral with respect to $S U(3) \times S U(2) \times U(1)$ and their may be additional fermions, chiral with respect to $G_{\mathrm{S}}$, that become non-chiral after the reduction from $G_{\mathrm{S}}$ to $S U(3) \times S U(2) \times$ $U(1)$.

The group $G_{\mathrm{S}}$ is the complete CP-group from the open sector times any gauge group generated by closed sector vector bosons. The physical realization of the group theoretical "reduction" mentioned above can take many forms, such as mass generation for $U(1)$ 's by Green-Schwarz anomaly cancelling terms, confinement or breaking by a fundamental or dynamical Higgs effect. Furthermore part of $G_{\mathrm{S}}$ may remain unbroken, if the corresponding gauge bosons do not couple to quarks and leptons. We did not commit ourselves here to a particular reduction mechanism. In the majority of the spectra we consider, $G_{\mathrm{S}}$ is embedded in $G_{\mathrm{SM}}$ as $G_{\mathrm{S}}=G_{\mathrm{SM}} \oplus G_{\text {Hidden }}$ (as a Lie-algebra), except in types 4 and 5 , where

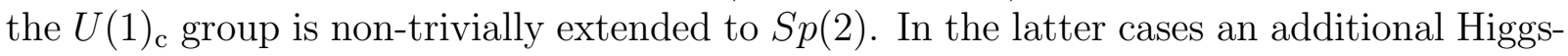
like mechanism would be required to arrive at the standard model. Just as is the case with the supersymmetry breaking and the standard model Higgs mechanism, one could impose additional constraints on the results in order for a a particular mechanism to be realized, but such constraints are less robust and more model-dependent than the requirement of chirality, our main guiding principle.

The possibilities for $G_{\mathrm{S}}$-chiral particles that are $G_{\mathrm{SM}}$-non-chiral are the following

1. Right-handed neutrinos. These particles are singlets (and hence not chiral) w.r.t. $G_{S M}$ but are chiral with respect to lepton number, which is broken. In our case, there are always three of them. This is an inevitable consequence of tadpole cancellation, which cancels the cubic anomalies in $U(1)_{\mathrm{d}}$, plus the fact that we only allow bifundamentals of the $(\mathrm{a}, \mathrm{b}, \mathrm{c}, \mathrm{d})$ branes to be chiral.

2. Higgsinos. In the MSSM the fermionic partners of the Higgs are non-chiral with respect to $G_{S M}$, but in models of types 1,3 and 5 there is a possibility for the Higgses to be chiral with respect to $U(2)_{\mathrm{b}}$. This gauge symmetry breaks to $S U(2)$ in the first step, but its initial presence can still forbid the generation of large masses for the Higgs. This is a desirable feature, as it may give a mechanism for getting light Higgs bosons. 
3. Mirror quarks and leptons, which are chiral with respect to $U(2)_{\mathrm{b}}$. These particles can appear for the same reason as the Higgsinos, but are less desirable. For example the $U(3) \oplus U(2)$ combinations $(3,2)+\left(3^{*}, 2\right)$ is chiral, but becomes non-chiral when $U(2)_{\mathrm{b}}$ is reduced to $S U(2)$. We have allowed such particles in principle, but (just as the chiral Higgsinos), they occur only rarely.

4. $G_{S M}$ singlets, which are chiral with respect to the hidden gauge group. Such particles couple only the the SM-matter gravitationally, and hence are acceptable as "dark matter", if not too abundant. Furthermore they may acquire a mass and/or be confined by $G_{\text {Hidden }}$ dynamics.

Unwanted chiral matter within the standard model sector can be avoided by the selection of a,b,c and d branes, and chiral matter from open strings stretching between the SM branes and the hidden branes can be avoided by appropriate selection of the latter. One could in principle also forbid chiral rank- 2 tensors within $G_{\text {Hidden }}$ by an a priori constraint, but it is very hard to forbid chiral bifundamentals within $G_{\text {Hidden }}$, except by constructing all solutions and eliminating them a posteriori. Since constructing all solutions is nearly impossible in most cases, we decided to allow $G_{\mathrm{SM}}$-singlets that are chiral with respect to $G_{\text {Hidden }}$. Nevertheless, they occur in only a small fraction (about 12.5\%) of our solutions. This is largely due to the fact that our search is biased in favor of few additional branes, and it is harder to make chiral spectra with fewer branes, and impossible with a single brane.

\subsubsection{Scope of the search}

In this paper we consider all modular invariant partition functions (MIPFs) that are symmetric simple current modifications of the charge conjugation invariant of all 168 minimal $\mathrm{N}=2$ tensor products. The precise number of such MIPFs is determined as follows. Generically, it is just a matter of applying the procedure of $[81,110]$ and restricting to symmetric bi-homorphisms $X$ (defined more precisely in [110] and in the next chapter). However, if there are identical $\mathrm{N}=2$ factors in the tensor product, there will be equivalences among these MIPFs, and we remove equivalent ones. Furthermore, for small values of $k$ (the "level" of the minimal model), especially $k=2$, generically distinct simple current invariants are in fact identical, and these are also removed from the set. We then end up with 5403 distinct MIPFs. They can be characterized in part by the resulting Hodge numbers $h_{11}$ and $h_{21}$, and by the number of gauge singlets in the heterotic string spectrum. These numbers can be compared to tables of such spectra produced about fifteen years ago $[134,72]$. Unfortunately a complete comparison is difficult, because the old results are either no longer available, and certainly not in electronic form, or the search was not fully exhaustive, or the symmetry of the MIPFs was not specified. But to the extent that a comparison is possible the results seem to agree.

In a few cases there appear to be further equivalences, or at least some MIPFs may correspond to distinct rational points on the same Calabi-Yau space. In total we found 
873 different combinations of Hodge numbers, and 1829 Hodge numbers plus singlets (i.e gauge singlets in the Heterotic spectrum.)

For each of these MIPFs we consider all orientifolds allowed by the general formula of [69]. These orientifolds are subject to three equivalence relations, one originating from permutations of identical factors, and two as part of the general formalism. These equivalences are removed, and we then end up with a total of 49322 a priori distinct orientifolds. Our results indicate that indeed they are generically distinct.

For each MIPF and orientifold we consider the complete set of boundaries. This means that the number of boundaries is equal to the number of Ishibashi states of the MIPF. The MIPF may be of any type: pure fusion rule automorphisms, extensions of the chiral algebra, or combinations thereof. The beauty of the formalism of [69] is that it works independently of such details. It is well-known that in the case of extensions one can distinguish boundaries that respect the extended symmetry and boundaries that do not. A complete set of boundaries contains both kinds. The CFT we start with is itself an extension of the minimal model tensor product CFT (by alignment currents and the spacetime supersymmetry current). Those extensions are respected by all our solutions, by construction. If the MIPF extends the chiral algebra further, one could work directly in the extended CFT and only consider boundaries that respect the extension. One would then find a subset of our solutions. Alternatively, one could also start with less symmetry, and treat for example space-time supersymmetry as a bulk extension. This would allow, in principle, supersymmetry breaking boundaries. In practice this is quite hard, because the number of primary fields increases dramatically. Undoubtedly, so will the number solutions.

Our goal was to complete this analysis for all MIPFs, in order to arrive at a picture that is as complete as possible. Unfortunately, the analysis could not be completed in all cases. Two tensor combinations had too many primaries to finish the computation of chiral intersections in a reasonable amount of time. In five others the number of candidate (a,b,c,d) branes was so large that we decided to restrict ourselves to types 0 and 1, of which there are fewer. For a given MIPF, orientifold and type, the number of four-stack candidates was more than a million in some cases.

The main computational stumbling block are the tadpole equations. For every valid set of $(a, b, c, d)$ branes, the number of variables is equal to the number of boundaries that do not have a chiral intersection with a,b,c and d. This number can become as large as several hundreds, for a few tens of tadpole equations. Obviously, the time needed to evaluate this completely grows exponentially with the surplus of variables over equations. This means that beyond a certain number of variables it is impossible to decide conclusively that there are no solutions. In those cases we did perform a systematic search for solutions with 0,1 and 2 hidden branes, and 3 if the number of variables was less than 100, 4 if the number was less than 400. Furthermore, in the simpler cases we attempted solving the equations in general. Of the 5403 MIPFs, 2 were not analysed at all, and 20 only for types 0 and 1 , and in 495 cases the tadpole equations were not fully analysed (153 of these did have solutions, however).

There are several possible ways to count solutions as distinct. On the one hand, one 
could regard solutions as identical if they are connected to each other by continuous, nonsingular variations of open or closed string moduli. In an RCFT approach this is hard to do, since we cannot vary the moduli in a continuous way. The other extreme would be to count all distinct massless spectra, including the hidden sector gauge groups and representations. This is also not possible in our case, since we did not do a systematic search through all hidden sector gauge groups. We have chosen an intermediate criterium: solutions are regarded as distinct if they are of different type or have a different massless (chiral and non-chiral) standard model spectrum. Furthermore we treat different dilaton couplings for the a,b,c,d branes and the O-plane as a distinction, and the absence or presence of a hidden sector. By contrast, in [59] hidden sector distinctions were also counted. The number of solutions for one of the MIPFs of the tensor product $(6,6,6,6)$ quoted in that paper ("more than 6000 ") reduces to 820 with our present way of counting.

\subsubsection{Contents}

This paper is organized as follows. In the next chapter, we review the ingredients of the algebraic orientifold construction. Section 4.3 contains a general discussion of the massless spectrum. In section 4.4 we discuss tadpole and anomaly cancellation. Chapter 4.5 contains our results. Due to the huge number of solutions it is impossible and pointless to present detailed spectra. Therefore we only give distributions of several quantities of interest, such as the number of Higgs scalars. We also analyse the values of gauge coupling ratios at the string scale. In section 4.6 we present one example in more detail, a model without any additional branes that in several respects is the simplest we encountered. In section 4.7 we formulate some conclusions. An essential computational technique is to organize the Ishibashi and boundary labels into simple current orbits, which leads to a dramatic speed-up of the calculations. This is explained in the appendix.

\subsection{Algebraic Model Building}

Our starting point is four-dimensional type-II string obtained by tensoring NSR fermions with a combination of $\mathrm{N}=2$ minimal models with total central charge 9 . In a covariant description, the NSR part of the theory is built out of four fermions $\psi^{\mu}$ with Minkowski metric, and a set of superconformal ghosts. The type-II theory is modular invariant and has two world-sheet supersymmetries needed for consistency. We assume it to be symmetric in left- and right-moving modes and have an extended chiral algebra leading to two space-time supersymmetries. To this theory we apply the orientifold procedure. Since our approach is based on unitary rational CFT, it is convenient to use this description not only for the minimal $\mathrm{N}=2$ factors, but also for the NSR part of the theory. To do so we use a bosonic description of the latter (see [113] and references therein). This is convenient because model-independent complications due to the GSO projection, spin-statistics and superconformal ghosts are automatically taken care of. This implies that we are formally constructing bosonic open strings. To obtain the spectrum we mimic the procedure used 
in the covariant approach, namely fix a ghost charge to select the physical states. This translates into a consistent truncation of the bosonic string characters. In the case of closed strings, this procedure can be shown to map modular invariant bosonic strings to modular invariant fermionic strings. In the case of open strings, it leads to fermionic open strings satisfying all the integrality conditions on torus and Klein bottle as well as Annulus and Möbius strip amplitudes, and that have the correct spin-statistics for all physical states, and the proper symmetrization for Ramond-Ramond states. We emphasize that this is only used here as a bookkeeping device, and that we are not trying to conjecture a relation between fermionic and bosonic strings.

Our starting point is a class of bosonic string theories with chiral algebra

$$
E_{8} \otimes D_{5} \otimes \mathcal{A}_{\text {int }}
$$

where $E_{8}$ and $D_{5}$ are level 1 affine Lie algebras models. In this paper we can take the model-dependent factor $\mathcal{A}_{\text {int }}$ to be of $4 d$ Gepner type

$$
\mathcal{A}_{\text {int }}=\otimes_{k=1}^{r} \mathcal{A}_{k} \quad, \quad c_{\text {int }}=9
$$

and $\mathcal{A}_{k}$ is the $N=2$ minimal model at level $k$. The $E_{8}$ factor has no influence on the massless spectrum. The only role of this factor is to cancel the conformal anomaly $c_{8}+c_{5}+c_{\text {int }}+c_{\text {bos }}+c_{\text {ghosts }}=8+5+9+4-26=0$ where $c_{\text {bos }}$ is the contribution of the uncompactified bosons. The $D_{5}$ factor describes the lightcone NSR fermions plus the longitudinal NSR fermions and superconformal ghosts. The truncation that we implement at the end of the day basically amounts to removing the contribution of the latter. For the construction of the type-II string we follow the procedure explained in [134] (see also [78]) for heterotic strings, the only difference being that the fermionic truncation is applied to both the left- and the rightmoving sector. The tensor product (4.1) is first extended by means of alignment currents (even combinations of the world-sheet supercurrents of the factors), needed to maintain $N=1$ world-sheet supersymmetry. To get theories with $N=2$ space-time supersymmetry we must extend the algebra (4.1) further by the simple current group generated by the currents $(0, S, S, S, S, \ldots)$, where $S$ is a spinor representation of $D_{5}$ or a Ramond ground state of each minimal model that is a simple current (in $D_{5}$ and each of the minimal models there are two choices, but which one we take is irrelevant as long as the same choice is made in both chiral sectors. For the minimal models we choose $(l, q, s)=(0,1,1)$, in the usual notation $)$.

Because all aforementioned chiral algebra extensions are of simple current type, all chiral data like the spectrum of primaries $\{i\}$, the conformal weights $h_{i}$, the modular matrix $S_{i j}$ and the fixed point resolution matrices $S^{J}$ can be expressed in terms of the chiral data of the original unextended tensor product.

In this paper use a left-right symmetric extension in order to be able to apply the boundary/crosscap state formalism of [69]. There is a second, asymmetric choice obtained by using instead of $(0, S, S, S, S, \ldots)$ the simple current $(0, C, S, S, S, \ldots)$ in of the chiral sector. These are called type IIB (symmetric) and type IIA (asymmetric) extensions respectively. 
After applying these extension one obtains 168 [114] four-dimensional type-IIB theories. Most of these have $N=2$ space-time supersymmetry, which will be broken to $N=1$ by the orientifold procedure. Five of the 168 theories have $N=4$ supersymmetry, and can be ignored for further purposes, as they will never yield chiral $N=1$ open strings. We treat all these theories as non-supersymmetric CFTs. From the world-sheet point of view, the world-sheet supercurrents are fields with conformal weight $\frac{3}{2}$ which are not in the chiral algebra (although their even powers are), and the space-time supercurrents are extended chiral algebra currents with conformal weight 1 , which are treated just as any other extension. There is one reason why conformal weight 1 currents are special, and that is that the sub-algebra they generate is an affine Lie-algebra or $U(1)$ factor. In this case they extend $D_{5}$ to $E_{6}$ (or $E_{7}$ in the $N=4$ theories). In this way we obtain and RCFT with $N_{\text {prim }}$ primaries, whose ground states are in some representation of $E_{6}$, of the general form $m_{0}(\mathbf{1}) \oplus m^{+}(\mathbf{2 7}) \oplus m^{-}\left(\mathbf{2 7}^{*}\right)$. For the $N=2$ theories, $N_{\text {prim }}$ varies between 260 and 108612 .

These CFTs are our starting point and their chiral algebras are left unbroken in the rest of the procedure. We will refer to it as the susy chiral algebra. As was discussed in [78], one could consider the possibility of starting with a smaller chiral algebra, and allow for the possibility that - for example - space-time supersymmetry is present in the bulk, but broken on some of the branes. While it is possible in principle to investigate this in our formalism, the practical problem is that the chiral algebra becomes smaller, and hence the number of primaries much larger.

Among the $N_{\text {prim }}$ primaries there is almost always a subset $N_{\text {sim }}$ that are simple currents. This number ranges from 2187 (equal to $N_{\text {prim }}$ ) for the tensor product $(1)^{9}$ to just 1. Under fusion, they form a discrete group $\mathcal{G}$. These simple currents are used to build symmetric modular invariant partition functions.

For a simple current MIPF one has to specify the following data

- A group $\mathcal{H}$ that consists of simple currents of $\mathcal{A}$. All currents $J$ in $\mathcal{H}$ must satisfy the condition that the product of their conformal weight $h_{J}$ and order $N_{J}$ is integer. In general $\mathcal{H}$ is a product of cyclic factors $\mathcal{H}=\prod_{\alpha} \mathbb{Z}_{N_{\alpha}}$. The generator of the $\mathbb{Z}_{N_{\alpha}}$ will be denoted as $J_{\alpha}$.

- A symmetric matrix $X_{\alpha \beta}$ that obeys

$$
\begin{aligned}
2 X_{\alpha \beta} & =Q_{J_{\alpha}}\left(J_{\beta}\right) \quad \bmod 1, \alpha \neq \beta \\
X_{\alpha \alpha} & =-h_{J_{\alpha}}
\end{aligned}
$$

plus a further constraint $N_{\alpha} X_{\alpha \beta} \in \mathbb{Z}$ for all $\alpha, \beta$.

Here $Q$ is the simple current monodromy charge, $Q_{J}(a)=h(a)+h(J)-h(J a)$, where $h$ is the conformal weight. When in the following we write $X\left(J, J^{\prime}\right)$ for arbitrary simple currents in $\mathcal{H}$ we mean

$$
X\left(J, J^{\prime}\right)=\prod_{\alpha, \beta} n_{\alpha} m_{\beta} X_{\alpha \beta}
$$


for $J=\prod_{\alpha} J_{\alpha}^{n_{\alpha}}$ and $J^{\prime}=\prod_{\alpha} J_{\alpha}^{m_{\alpha}}$.

The resulting value of $Z_{i j}$ is the number of currents $L \in \mathcal{H}$ such that

$$
\begin{aligned}
j & =L i \\
Q_{M}(i)+X(M, L) & =0 \bmod 1
\end{aligned}
$$

for all $M \in \mathcal{H}$. These MIPFs can be further chiral algebra extensions of the susy chiral algebra, fusion rule automorphisms or combinations thereof. The formalism of [69] is insensitive to the distinction among these various types. With the exception of certain pathological cases, this set of MIPFs is the most general one where the combinations $(i, j)$ of left and right representations that occur are linked by simple currents, i.e. $i=J j^{c}$ for some $J \in c H$. As the "c" indicates, we build simple currents starting from the charge conjugation invariant. One could also start from the diagonal invariant, but there is no general formula available for the boundary and crosscap coefficients in that case. In many (though not all) cases the diagonal invariant is itself a simple current automorphism of the charge conjugation invariant, and hence is already included. It is well-known that additional, "exceptional" MIPFs exist for the Gepner models (see [133], [80]), including the famous "three-generation" one for the tensor product $(1,16,16,16)$ [82], but again there is no boundary/crosscap formalism available for these cases (although the boundary coefficient are known for the $S U(2)$ exceptional invariants [20]).

The next step is to specify the orientifold data, which consist of

- A Klein bottle current $K$. This can be any simple current of $\mathcal{A}$ that is local with all order two currents in $\mathcal{H}$, i.e., obeys

$$
Q_{I}(K)=0 \quad \bmod 1 \forall I \in \mathcal{H}, I^{2}=0 .
$$

- A set of phases $\beta_{K}(J)$ for all $J \in \mathcal{H}$ that satisfy

$$
\beta_{K}(J) \beta_{K}\left(J^{\prime}\right)=\beta_{K}\left(J J^{\prime}\right) e^{2 \pi i X\left(J, J^{\prime}\right)} \quad, J, J^{\prime} \in \mathcal{H}
$$

with $\beta_{K}(J)=e^{i \pi\left(h_{K L}-h_{K}\right)} \eta(K, L)$, with $\eta(K, L)= \pm 1$.

Note that the phases $\beta_{K}(J)$ satisfy the same multiplication rule independent of the Klein bottle current $K$, and that the solutions depend on $K$ because of the second requirement. The signs $\eta$ can be chosen freely provided the multiplication rule for $\beta$ holds. It is easy to see that this implies that there is a freedom of choosing one sign for each independent even factor in the simple current subgroup $\mathrm{cH}$. All these choices yield valid orientifolds, but they are some equivalences, which will be discussed below.

This data defines a (bosonic) orientifold with spectrum encoded in the total one-loop partition function

$$
\frac{1}{2}(\mathcal{T}+\mathcal{K}+\mathcal{A}+\mathcal{M})
$$


where we distinguish between the four topologically distinct surfaces with vanishing Euler number. These contributions can be expanded in (bi)linears of (hatted) characters in the usual way [8]:

$$
\begin{array}{rlrl}
\mathcal{T} & =\sum_{i j} Z_{i j} \chi_{i} \chi_{j}^{*}, & \mathcal{K} & =\sum_{i} K^{i} \chi_{i} \\
\mathcal{A} & =\sum_{a b} \mathcal{N}_{a} \mathcal{N}_{b} A^{i}{ }_{a b} \chi_{i} & \mathcal{M} & =\sum_{a} \mathcal{N}_{a} M^{i}{ }_{a} \hat{\chi}_{i}
\end{array}
$$

The following objects are introduced:

- The labels $a, b$ that appear in the open string sector of the partition function are a short-hand notation for the boundary labels. In full glory these labels are $\mathcal{H}$-orbits $[a]$ of a chiral sector $a$ with a possible degeneracy label $\psi_{a}$ which is a (discrete group) character of the a certain subgroup of the stabilizer, called the central stabilizer $\mathcal{C}_{a}$ (see [69] and below). We write this as $\left[a, \psi_{a}\right]$.

- The nonnegative integers $\mathcal{N}_{a}:=\mathcal{N}_{\left[a, \psi_{a}\right]}$ are the CP-factors. These numbers must be such that the total partition function is free of divergences. This will be reviewed in section 4.4.1.

The Klein bottle, annulus and Möbius coefficients factorize as

$$
\begin{aligned}
K^{i} & =\sum_{m, J, J^{\prime}} \frac{S_{m}^{i} U_{(m, J)} g_{J, J^{\prime}}^{\Omega, m} U_{\left(m, J^{\prime}\right)}}{S_{0 m}} \\
A_{\left[a, \psi_{a}\right]\left[b, \psi_{b}\right]}^{i} & =\sum_{m, J, J^{\prime}} \frac{S_{m}^{i} R_{\left[a, \psi_{a}\right](m, J)} g_{J, J^{\prime}}^{\Omega, m} R_{\left[b, \psi_{b}\right]\left(m, J^{\prime}\right)}}{S_{0 m}} \\
M_{\left[a, \psi_{a}\right]}^{i} & =\sum_{m, J, J^{\prime}} \frac{P_{m}^{i} R_{\left[a, \psi_{a}\right](m, J)} g_{J, J^{\prime}}^{\Omega, m} U_{\left(m, J^{\prime}\right)}}{S_{0 m}}
\end{aligned}
$$

where $P=\sqrt{T} S T^{2} S \sqrt{T}[118]$ and $S, T$ are the usual modular matrices. In these expressions the sums run over all Ishibashi labels. These labels are pairs $(m, J)$ that obey

$$
\begin{array}{r}
m=J m, \\
Q_{K}(m)+X(M, J)=0 \quad \bmod 1
\end{array}
$$

for all $M \in \mathcal{H}$. We note that we consider boundaries and crosscaps of "trivial automorphism type" [121], which means that we require that the susy chiral algebra is preserved (and not just preserved up to automorphism) in closed string scattering from one of these defects. This implies that the closed strings that can couple to these defects must be Cdiagonal. The Ishibashi labels (4.8) are one-to-one to such closed string sectors. We have also introduced 
- The Ishibashi metric $g^{\Omega, m}$

$$
g_{J, J^{\prime}}^{\Omega, m}=\frac{S_{m 0}}{S_{m K}} \beta_{K}(J) \delta_{J^{\prime}, J^{c}}
$$

for all $J, J^{\prime} \in \mathcal{H}$. Here $\Omega$ indicates the choice of Klein bottle current as well as the phases $\beta_{K}(J)$ that define an orientifold.

- The boundary reflection coefficients

$$
R_{\left[a, \psi_{a}\right](m, J)}=\sqrt{\frac{|\mathcal{H}|}{\left|\mathcal{C}_{a}\right|\left|\mathcal{S}_{a}\right|}} \psi_{a}^{*}(J) S_{a m}^{J}
$$

- The crosscap reflection coefficients

$$
U_{(m, J)}=\frac{1}{\sqrt{|\mathcal{H}|}} \sum_{L \in \mathcal{H}} \eta(K, L) P_{L K, m} \delta_{J, 0}
$$

where $S^{J}$ is the fixed point resolution matrix $S^{J}$, whose rows and columns are labelled by fixed points $a, m$ o f $J$, implements a modular $S$-transformation on the torus with $J$ inserted. It is unitary and obeys [71]

$$
S_{K i, j}^{J}=F_{i}(K, J) e^{2 \pi i Q_{K}(j)} S_{i j}^{J}
$$

The aforementioned central stabilizer is defined in terms of this quantity as

$$
\mathcal{C}_{a}=\left\{J \in \mathcal{S}_{a} \mid F_{a}(K, J) e^{2 \pi i X(K, J)}=1 \text { for all } K \in \mathcal{S}_{a}\right\}
$$

One can check that $R$ is unitary. The reflection coefficients have an important physical meaning, because they are (proportional to) the coupling of closed strings from Ishibashi sector $(m, J)$ to D-brane $\left[a, \psi_{a}\right]$. The oriented annulus amplitude therefore reads

$$
\left[A^{\mathrm{or}}\right]_{\left[a, \psi_{a}\right]}^{i \quad\left[b, \psi_{b}\right]}:=\sum_{m, J, J^{\prime}} \frac{S_{m}^{i} R_{\left[a, \psi_{a}\right](m, J)} R_{\left[b, \psi_{b}\right]\left(m, J^{\prime}\right)}^{*}}{S_{0 m}}
$$

In the unoriented string, specified by the Klein bottle current $K$ and the phases $\beta(J)$, the annulus amplitude is

$$
A_{\left[a, \psi_{a}\right]\left[b, \psi_{b}\right]}^{i}=\left[A^{\text {or }}\right]_{\left[a, \psi_{a}\right]}^{i}\left[b_{b}\right]^{c}
$$

where $\left[b, \psi_{b}\right]^{c}$ is the conjugate boundary label. Geometrically the pair of branes $\left[b, \psi_{b}\right]$ and $\left[b, \psi_{b}\right]^{c}$ are mapped to each other by the orientifold action $\Omega \mathcal{R}=\Omega \mathcal{R}(K, \beta)$. In CFT this image is encoded by the boundary conjugation matrix

$$
A_{\left[a, \psi_{a}\right]\left[b, \psi_{b}\right]}^{0}= \begin{cases}1 & ,\left[b, \psi_{b}\right]=\left[a, \psi_{a}\right]^{c} \\ 0 & , \text { otherwise }\end{cases}
$$


Note that the various unoriented annuli can all be obtained from the unique oriented annulus by multiplication with the boundary conjugation matrix.

The physical meaning of $U$ is as coupling constants between the Ishibashi sectors $(m, J)$ and the O-plane.

This formalism has been shown to lead to integer values for all open and closed string particle multiplicities [96]. This results holds for all RCFTs, not just the minimal $\mathrm{N}=2$ models considered here. This universal validity gives additional confidence in its correctness, but the ultimate consistency check would be to demonstrate that all sewing constraints are satisfied on all Riemann surfaces. This has been done for orientable surfaces [73], and is underway for the non-orientable case.

\subsubsection{Orientifold equivalences}

For a general simple current MIPF the set of known orientifolds is parametrized by a Klein bottle current $K$ and a number of signs $\epsilon$, one for each independent even factor in the discrete group that defines the MIPF. The Klein bottle current can be any simple current subject to the constraint (4.2), and there is no restriction on the signs. However, not all these choices are inequivalent. The following equivalences exist between the choices $\{K, \epsilon\}$ (here $\mathcal{G}$ is the full group of simple currents and $\mathcal{H}$ the subgroup used in the construction of the MIPF)

$$
\begin{aligned}
& \{K, \epsilon\} \sim\left\{K J^{2}, \epsilon^{\prime}\right\}, \quad J \in \mathcal{G} \\
& \{K, \epsilon\} \sim\left\{K L, \epsilon^{\prime \prime}\right\}, \quad L \in \mathcal{H} \\
& \{K, \epsilon\} \sim\{\pi(K), \quad \hat{\pi}(\epsilon)\}
\end{aligned}
$$

Here $\pi$ is the action induced by the permutation of identical minimal models (if any) on the primary fields of the tensor product, and $\hat{\pi}$ is the action induced on the signs $\epsilon$. The modified signs $\epsilon^{\prime}$ and $\epsilon^{\prime \prime}$ can be worked out from the formula for the crosscap coefficients (4.10) and the relation

$$
P_{J^{2} a, b}=\epsilon_{J^{2}}(a) e^{2 \pi i\left[Q_{J}(b)-Q_{J}(J a)\right]} P_{a b} ; \quad \epsilon_{J}(a)=e^{\pi i\left[h_{a}-h_{J a}\right]},
$$

but we will not present them explicitly here. The combined action of the three equivalences organizes the various crosscap choices into equivalence classes, and we have taken into account one representative from each class. The results seem to indicate that there are no further equivalences: in general the number of (a,b,c,d) stacks of various types, as well as the number of tadpole solutions is distinct.

Note that in each case the equivalence between orientifolds holds up to a certain permutation of the boundary labels. A subgroup of these transformation may fix the orientifold, but lead to a residual equivalence of boundary choices for a given orientifold. We did not attempt to remove this (and other) equivalence among boundaries, because it was much easier to compare the resulting spectra and remove identical ones a posteriori. 


\subsection{Massless Spectrum}

Our prime interest will be the identification of the massless states in the partition function (4.3). Before doing this in the correct way, we have to perform a truncation to obtain the spectrum of the superstring. This truncation is most easily described as follows. Note that the current $\left(0_{8}, S_{5}, S\right)$ has spin 1 and that therefore the bosonic algebra $\mathcal{A}$ must contain a level one WZW factor that is larger than $D_{5}$. The only possibility is $E_{6}$. All primaries $m$ of $\mathcal{A}$ therefore decompose into primaries of $E_{6}$. Tachyonic states are always singlets of $E_{6}$. Massless states are singlets, fundamentals $\mathbf{2 7}$, anti-fundamentals $\mathbf{2 7}$ * or adjoints $\mathbf{7 8}$. The truncation from the bosonic spectrum to a superstring spectrum is

$$
\begin{aligned}
\text { left-movers } \quad & \rightarrow- \\
& \mathbf{2} 7 \rightarrow \frac{1}{2} \Psi \quad, \quad \mathbf{2 7 ^ { * }} \rightarrow \frac{1}{2} \Psi^{*} \\
& \mathbf{7 8} \rightarrow V \\
\text { right-movers } & \rightarrow- \\
\mathbf{2 7} & \rightarrow \frac{1}{2} \Psi \quad, \quad \mathbf{2 7 ^ { * }} \rightarrow \frac{1}{2} \Psi^{*} \\
\mathbf{7 8} & \rightarrow V
\end{aligned}
$$

where $\Psi$ is a (complex) $N=1$ chiral multiplet and $V$ a $N=1$ vector multiplet. Note that $E_{6}$ singlets are projected out. The $\mathbf{2 7}$ representation thus yields one real bosonic degree of freedom, and one fermionic one. In heterotic strings a representation $(\mathbf{2 7}, \mathbf{R})$ (where $R$ is some gauge representation) is always accompanied by a $\left(\mathbf{2 7}, \mathbf{R}^{*}\right)$, and together they form one $N=1$ chiral multiplet, containing a complex boson and a Weyl fermion in the representation $R$. In type-II closed strings, the combinations $(\mathbf{2 7}, \mathbf{2 7})+\left(\mathbf{2 7 ^ { * }}, \mathbf{2 7 ^ { * }}\right)$ yields one $\mathrm{N}=2$ vector multiplet, with four real bosonic and four real fermionic degrees of freedom. The combination $\left(\mathbf{2 7}, \mathbf{2 7} \mathbf{7}^{*}\right)+\left(\mathbf{2} \mathbf{7}^{*}, \mathbf{2 7}\right)$ yields one $N=2$ hyper multiplet, with the same number of degrees of freedom. Note that in principle one could switch the rôle of the $\mathbf{2 7}$ and the $\mathbf{2} \mathbf{7}^{*}$ in the truncations for the right-movers with respect to the left-movers. In covariant language, this corresponds to switching the ghost-charge assignment for the fermions. This would map a IIA spectrum to a IIB spectrum and vice-versa. However, the same interchange can also be achieved by going from a IIB to a IIA extension. In applications to orientifolds, it is clearly preferable to adopt a universal truncation rule (ghost charge assignment) for left and right-moving, as well as open string characters.

To every $h_{i}=1$ primary we can associate a Witten index

$$
w_{i}=m_{i}^{+}-m_{i}^{-}
$$

where $m_{i}^{+}\left(m_{i}^{-}\right)$counts the number of $\mathbf{2 7}\left(\mathbf{2 7 ^ { * } )}\right.$ in $i$. Note that

$$
m_{i^{c}}^{ \pm}=m_{i}^{\mp} \rightarrow w_{i^{c}}=-w_{i}
$$




\subsubsection{The Oriented Closed String Spectrum}

The torus contribution $\mathcal{Z}$ in (4.3) is the partition function of the parent theory of the orientifold. After undoing the bosonic string map it describes type II string theory on some Calabi-Yau 3-fold at the Gepner point. The ground state of the vacuum sector $(00)$ is projected out. At the first excited level it yields

$$
V * V=G+H
$$

the $N=2$ gravity and universal hyper multiplet. The other massless sectors yield the model-dependent Abelian vector and hyper multiplets. First note that modular invariance implies

$$
Z_{i j}=Z_{i^{c} j^{c}}
$$

Complex sectors $(i j)+\left(i^{c} j^{c}\right)$ contribute the following $N=2$ multiplets

$$
\begin{array}{ll}
Z_{i j}\left(m_{i}^{+} m_{j}^{+}+m_{i}^{-} m_{j}^{-}\right) & \text {vector multiplets } \\
Z_{i j}\left(m_{i}^{+} m_{j}^{-}+m_{i}^{-} m_{j}^{+}\right) & \text {hyper multiplets }
\end{array}
$$

Real sectors $(i j), i=i^{c}, j=j^{c}$ contribute

$$
\begin{array}{ll}
Z_{i j} m_{i}^{+} m_{j}^{+} & \text {vector multiplets } \\
Z_{i j} m_{i}^{+} m_{j}^{+} & \text {hyper multiplets }
\end{array}
$$

The total numbers $h_{21}$ of vector multiplets and $h_{11}$ of hyper multiplets are

$$
\begin{aligned}
h_{21} & =\frac{1}{2} \sum_{i j} Z_{i j}\left(m_{i}^{+} m_{j}^{+}+m_{i}^{-} m_{j}^{-}\right) \\
h_{11} & =\frac{1}{2} \sum_{i j} Z_{i j}\left(m_{i}^{+} m_{j}^{-}+m_{i}^{-} m_{j}^{+}\right) .
\end{aligned}
$$

The sum is over all fields, including conjugates, and the factor $\frac{1}{2}$ corrects for doublecounting; for real fields $m_{i}^{+}=m_{i}^{-}$. Note that

$$
\chi:=2\left[h_{21}-h_{11}\right]=\sum_{i j} w_{i} w_{j^{c}} Z_{i j}
$$

is a topological invariant. The string theory we have constructed therefore has the same massless spectrum as type IIB string theory on a Calabi-Yau manifold $X_{3}$ with Hodge numbers $h_{11}$ and $h_{21}$.

The spectrum of the other dual pair, (IIA $/ X_{3}$, IIB $/ \tilde{X}_{3}$ ), can be obtained by conjugating the right-moving space-time supercurrent, which results in the IIA extension. It is easy to see that there are $h_{21}$ hyper multiplets and $h_{11}$ vector multiplets in this case.

\footnotetext{
${ }^{1}$ Note that in [59] the Hodge numbers of the type IIA compactification were listed. In this paper we list them for the type IIB compactification, the closed string theory to which we apply the orientifold procedure.
} 


\subsubsection{The Unoriented Closed String Spectrum}

The first step in the orientifold procedure is the truncation of the type II spectrum to states that are invariant under the involution $\Omega=\Omega(K, \beta)$. The resulting massless $d=4, N=1$ spectrum can be obtained from $\frac{1}{2}(\mathcal{Z}+\mathcal{K})$ by simple counting arguments. The vacuum sector gives the universal gravity multiplet and a chiral multiplet that contains the dilaton. Offdiagonal sectors do not flow in the direct Klein bottle. Their contribution is halved by the orientifold projection. Complex off-diagonal sectors $(i j)+\left(i^{c} j^{c}\right)+(j i)+\left(j^{c} i^{c}\right)$ contribute the following $N=1$ multiplets

$$
\begin{gathered}
Z_{i j}\left(m_{i}^{+} m_{j}^{+}+m_{i}^{-} m_{j}^{-}\right) \quad \text { vector multiplets } \\
Z_{i j}\left(m_{i}^{+} m_{j}^{+}+m_{i}^{-} m_{j}^{-}\right)+2 Z_{i j}\left(m_{i}^{+} m_{j}^{-}+m_{i}^{-} m_{j}^{+}\right) \quad \text { chiral multiplets }
\end{gathered}
$$

Real off-diagonal sectors $(i j)+(j i)$ contribute

$$
\begin{array}{cc}
Z_{i j} m_{i}^{+} m_{j}^{+} & \text {vector multiplets } \\
3 Z_{i j} m_{i}^{+} m_{j}^{+} & \text {chiral multiplets }
\end{array}
$$

Diagonal sectors are symmetrized or anti-symmetrized according to the Klein bottle coefficient. Complex diagonal sectors $(i i)+\left(i^{c} i^{c}\right)$ contribute

$$
\begin{gathered}
\frac{1}{2}\left(Z_{i i}-K_{i}\right)\left(m_{i}^{+} m_{i}^{+}+m_{i}^{-} m_{i}^{-}\right) \quad \text { vector multiplets } \\
\frac{1}{2}\left(Z_{i i}+K_{i}\right)\left(m_{i}^{+} m_{i}^{+}+m_{i}^{-} m_{i}^{-}\right)+Z_{i i}\left(m_{i}^{+} m_{i}^{-}+m_{i}^{-} m_{i}^{+}\right) \quad \text { chiral multiplets }
\end{gathered}
$$

Real diagonal sectors contribute

$$
\begin{gathered}
\frac{1}{2}\left(Z_{i i}-K_{i}\right) m_{i}^{+} m_{i}^{+} \quad \text { vector multiplets } \\
\frac{1}{2}\left(Z_{i i}+K_{i}\right) m_{i}^{+} m_{i}^{+}+Z_{i i} m_{i}^{+} m_{i}^{-} \quad \text { chiral multiplets }
\end{gathered}
$$

Define

$$
h_{11}^{ \pm}:=\frac{1}{4}\left[\sum_{i j}\left(Z_{i j} \pm \delta_{i j} K_{i}\right)\left(m_{i}^{+} m_{j}^{+}+m_{i}^{-} m_{j}^{-}\right)\right],
$$

where we sum over all primaries. Then the total number of closed string Abelian vector multiplets is $h_{11}^{-}$and the total number of model-dependent closed string chiral multiplets is $h_{21}+h_{11}^{+}$. In a geometrical setting the numbers $h_{11}^{+}$and $h_{11}^{-}$denote the number of harmonic $(1,1)$-forms that are anti-invariant or invariant under the orientifold action.

\subsubsection{The Oriented Open String Spectrum}

The massless gauge bosons of the $\Pi_{a} U\left(N_{a}\right)$ gauge group come from the first excited level of the vacuum sector. The annulus coefficient $A_{a}^{i b}$ counts states in the bifundamental $\left(V_{a}, V_{b}^{*}\right)$ representations of the space-time gauge group. Note that

$$
A_{a}^{i^{c} b}=A_{b}^{i a}
$$


Let $M_{a}^{+b}\left(M_{a}^{-b}\right)$ denote the number of chiral (anti-chiral) multiplets that transform according to $\left(V_{a}, V_{b}^{*}\right)$. These numbers are given by

$$
M_{a}^{ \pm b}=\sum_{i} m_{i}^{ \pm} A_{b}^{i a}
$$

where we sum over all primaries. Due to (4.12) the spectrum obeys the $d=4$ CPT relation $M_{b}^{ \pm a}=M_{a}^{\mp b}$. The net chirality is measured by the anti-symmetric chiral intersection matrix

$$
I_{a}^{b}=M_{a}^{+b}-M_{a}^{-b}=\sum_{i} w_{i} A_{a}^{i b}
$$

\subsubsection{The Unoriented Open String Spectrum}

The open string spectrum of the orientifold is encoded in $\frac{1}{2}(\mathcal{A}+\mathcal{M})$. The boundary conjugation matrix $A_{a b}^{0}$ defines the orientifold image or conjugate $a^{c}$ of brane $a$. Complex pairs of branes $a \neq a^{c}$ give rise to unitary gauge groups. For real branes $a=a^{c}$ the gauge group depends on the Möbius coefficient $M_{a}^{0}$. When $M_{a}^{0}=-1 /+1$, the first excited level of the vacuum is symmetrized/anti-symmetrized, signalling a $S p\left(N_{a}\right) / S O\left(N_{a}\right)$ gauge group. We can summarize this as

$$
G=\otimes_{a, \text { complex }} U\left(N_{a}\right) \otimes_{a, \text { real }} S O\left(N_{a}\right) \otimes_{a, \text { pseudo-real }} S p\left(N_{a}\right)
$$

The CP-factors $N_{a}$ are determined by tadpole cancellation (see subsection 4.4.1). In our conventions $A_{a b}^{i}$ counts states in $\left(V_{a}, V_{b}^{*}\right)$. When we conjugate a brane label, the corresponding vector must be conjugated. So $A_{a^{c} b}^{i}$ counts states in $\left(V_{a}^{*}, V_{b}^{*}\right)$ etcetera. Note that $^{2}$

$$
A_{a b}^{i^{c}}=A_{a^{c} b^{c}}^{i}
$$

For off-diagonal sectors $a \neq b$, the sectors $(a, b)$ and $(b, a)$ must be identified. The total number $M_{a b}^{+}\left(M_{a b}^{-}\right)$of chiral (anti-chiral) multiplets that transform according to $\left(V_{a}, V_{b}^{*}\right)$ is

$$
M_{a b}^{ \pm}=\sum_{i} m_{i}^{ \pm} A_{a b}^{i}
$$

where we sum over all primaries. Due to (4.13) the spectrum obeys the $d=4$ CPT relation $M_{a^{c} b^{c}}^{ \pm}=M_{a b}^{\mp}$. The net chirality of fermions transforming as $\left(V_{a}, V_{b}^{*}\right)$ is measured by

$$
\Delta_{a b}=M_{a b}^{+}-M_{a b}^{-}=\sum_{i} w_{i} A_{a b}^{i}
$$

Note that the ordering of the indices is irrelevant. From (4.13) we easily derive

$$
\Delta_{a b}=-\Delta_{a^{c} b^{c}} \quad, \quad \Delta_{a b^{c}}=-\Delta_{a^{c} b}
$$

\footnotetext{
${ }^{2}$ This follows from

$$
R_{a(m, J)}^{*}=g_{J J^{\prime}}^{\Omega, m} R_{a^{c}\left(m, J^{\prime}\right)}
$$

which can be derived from $A_{a b}^{i}=A_{a}^{i} b^{c}$, unitarity of $S$ and completeness.
} 
When $b=a^{c}$ the representations are adjoints (Adj) of $U\left(N_{a}\right)$. Of course, adjoint matter cannot give rise to chiral matter in $d=4$, as can easily be seen from (4.14). In order to make contact with geometry, we note that $\Delta_{a b}$ is the upper-half part of the geometric intersection matrix and that the lower-half is given by $\Delta_{a^{c} b^{c}}$. Diagonal sectors $a=b$ are projected by the Möbius strip to symmetric (S) or anti-symmetric (A) representations of the gauge group $G\left(N_{a}\right)$. Note that ${ }^{3}$

$$
M_{a}^{i^{c}}=M_{a^{c}}^{i}
$$

In a self-explanatory notation, the number of chiral (anti-chiral) multiplets in these representations are

$$
\begin{aligned}
& M_{a, \mathrm{~S}}^{ \pm}=\frac{1}{2} \sum_{i} m_{i}^{ \pm}\left(A_{a a}^{i}+M_{a}^{i}\right) \\
& M_{a, \mathrm{~A}}^{ \pm}=\frac{1}{2} \sum_{i} m_{i}^{ \pm}\left(A_{a a}^{i}-M_{a}^{i}\right)
\end{aligned}
$$

From (4.13) and (4.15) these multiplicities obey the CPT relations $M_{a, \mathrm{~S}}^{ \pm}=M_{a^{c}, \mathrm{~S}}^{\mp}$ and $M_{a, \mathrm{~A}}^{ \pm}=M_{a^{c}, \mathrm{~A}}^{\mp}$. The net chirality is

$$
\begin{aligned}
\Delta_{a, \mathrm{~S}} & =\frac{1}{2} \sum_{i} w_{i}\left(A_{a a}^{i}+M_{a}^{i}\right) \\
\Delta_{a, \mathrm{~A}} & =\frac{1}{2} \sum_{i} w_{i}\left(A_{a a}^{i}-M_{a}^{i}\right) .
\end{aligned}
$$

For real branes $a$, this index vanishes, as befits the symmetric and anti-symmetric representations of symplectic and orthogonal groups in $d=4$. When we compare with a geometric description, $\sum_{i} w_{i} A_{a a}^{i}$ is the intersection between a brane and its image, whereas $\sum_{i} w_{i} M_{a}^{i}$ is the intersection between a brane and the orientifold plane(s).

\subsection{Tadpoles \& Anomalies}

\subsubsection{Tadpole cancellation}

Non-vanishing one-point functions of massless scalars on the disk or crosscap may cause several problems. If the scalar is physical particle in the spectrum, surviving the orientifold projection, a tadpole indicates an instability in the vacuum. This manifests itself as an infinity in the Euler number 0 diagrams. In this case the theory would be unstable, but one might still hope that a stable vacuum exists. If the scalar is not a physical particle, the

\footnotetext{
${ }^{3}$ We now also need

$$
U_{(m, J)}^{*}=g_{J J^{\prime}}^{\Omega, m} U_{\left(m, J^{\prime}\right)}
$$
}


presence of a tadpole renders the theory inconsistent, and this may manifest itself through an uncanceled anomaly. If all Klein bottle coefficients are positive, all scalars from NS-NS sectors are physical, but since the R-R-sector always has a projection with opposite sign, the R-R-scalars are unphysical.

In a supersymmetric theory the NS-NS and R-R sectors are linked, and canceling unphysical tadpoles is equivalent to cancelling all tadpoles. The condition for the cancellation of all tadpoles is

$$
\sum_{b} N_{b} R_{b(m, J)}=4 \eta_{m} U_{(m, J)}
$$

for all Ishibashi labels $(m, J)$ for which the sector $\left(m m^{c}\right)+\left(m^{c} m\right)$ in the torus (4.4) yields massless space-time scalars. Here $\eta_{0}=1$ and -1 otherwise. Tadpole cancellation is a condition on the CP-factors $N_{a}$ of the gauge groups.

There are two further constraints on the CP multiplicities. If two boundaries $a$ and $b$ are conjugate, one must require that $N_{a}=N_{b}$, and if the CP-group associated with label $a$ is symplectic $N_{a}$ must be even.

The dilaton couplings $R_{0 b}$ are always positive (the Ishibashi label $m=0$ is nondegenerate, so there is no need for the degeneracy label $J$ ). Hence one can only satisfy the dilaton tadpole condition if $U_{0}<0$. The overall sign of the crosscap coefficients is a free parameter, which must be fixed so that $U_{0}<0$. Changing this sign changes the sign of all Möbius coefficients.

\subsubsection{Anomaly cancellation}

The chiral gauge anomalies can be obtained from a formal polynomial that is proportional to

$$
\mathcal{P}(F)=\sum_{i} w_{i}\left[\sum_{a b} A_{a b}^{i} \operatorname{ch}_{a}(F) \operatorname{ch}_{b}(F)+\sum_{a} M_{a}^{i} \operatorname{ch}_{a}(2 F)\right]
$$

where

$$
\operatorname{ch}_{a}(F):=\sum_{n} \frac{1}{n !} \operatorname{Tr}_{a} F^{n},
$$

where the trace in $\operatorname{Tr}_{a} F^{n}$ is taken over the fundamental representation of $U\left(N_{a}\right)$, and over the anti-fundamental in $\operatorname{Tr}_{a^{c}} F^{n} ; F$ is the field strength two-form. To obtain the cubic anomalies in four dimensions one restricts to polynomial to six-forms and applies the descent method. The argument $F$ can be expanded in a Lie-algebra basis with generators $T^{k}$ as $\sum_{k} F^{k} T^{k}$. If $F$ lies entirely within a real subalgebra all odd terms (in $F$ ) in the polynomial vanish, and in particular there are no four-dimensional anomalies.

Following [24], we can we can easily show that tadpole cancellation implies cancellation of the purely cubic terms in the polynomial, but not the others (the "purely cubic" terms are obtained by keeping only terms proportional to $\operatorname{Tr} F^{3}$, without using group-dependent factorizations of such terms (such factorizations of cubic traces exist only for $U(1)$ and $U(2))$. To do so, we use that fact that the chiral characters or Witten indices transform 
to themselves under modular transformations, as well as under transformations involving the $P$-matrix, because they are independent of the modulus $\tau$

$$
w_{i}=i \sum_{j} S_{j}^{i} w_{j} \quad, \quad w_{i}=i \sum_{j} P_{j}^{i} w_{j}
$$

substituting this into the cubic part of the polynomial, and using the expression for the annulus and Möbius strip amplitudes (4.6) and (4.7) we get

$$
\begin{aligned}
\mathcal{P}(F)_{\text {cubic }}=-i \sum_{m, J, J^{\prime}}\left(S_{0 m}\right)^{-1} & w_{m} \sum_{a} R_{(m, J) a} g_{J, J^{\prime}}^{\Omega, m} \\
& \times\left\{\sum_{b} R_{\left(m, J^{\prime}\right) b}\left[N_{a} \operatorname{Tr}_{b} F^{3}+N_{b} \operatorname{Tr}_{a} F^{3}\right]+U_{\left(m, J^{\prime}\right)}\left[8 \operatorname{Tr}_{a} F^{3}\right]\right\}
\end{aligned}
$$

Now we interchange the summed indices $a$ and $b$ in the second term, use the fact that $g_{J, J^{\prime}}^{\Omega, m}=g_{J^{\prime}, J}^{\Omega, m}$, and substitute for $U_{(m, J)}$ the righthand side of (4.16) (note that only terms with $m \neq 0$ contribute, because the vacuum sector is non-chiral in four dimensions), and we see immediately that $\mathcal{P}(F)_{\text {cubic }}=0$.

For non-abelian factors in the gauge group this implies simply the usual cancellation of cubic anomalies. For $U(1)$-factors that get chiral contributions only from vectors, it also has the expected consequence: the total number of vectors and conjugate vectors must be the same in each such factor. The situation is a bit more interesting as soon as chiral tensors contribute. For example, if we assign charge \pm 1 to the (anti)-fundamental representations of a $U(1)$ factor, then symmetric and anti-symmetric tensors have charge \pm 2 . The anomaly cancellation implied by tadpole cancellation has nothing to do with the third power of these charges, but is an extrapolation of $U(N)$ anomaly cancellation to $N=1$. Hence we get a contribution proportional to \pm 1 for vectors, $\pm(N-4)=\mp 3$ for anti-symmetric tensors and $\pm(N+4)= \pm 5$ for symmetric ones (note furthermore that anti-symmetric $U(1)$ tensors do not even exist; in a "massless" anti-symmetric sector the first state in the spectrum is a massive symmetric tensor). This is not a problem, because precisely for $U(1)$ factors the cubic traces factorizes into lower order traces, which are not cancelled by tadpole cancellation anyway, but are removed by the Green-Schwarz mechanism.

In this paper we only encounter vector/anti-vector anomaly cancellation within the standard model gauge groups because we have required that all chiral particles attached to the $(a, b, c, d)$ branes should be bifundamentals. This implies in particular the presence of three left-handed anti-neutrinos to cancel the $\mathrm{c}$ and d-brane anomalies. It might be worth considering to drop the restriction to bifundamentals for the $\mathrm{c}$ and $\mathrm{d}$ branes and achieve anomaly cancellation by means of tensors, but we will not pursue that possibility here.

In those cases where the b-brane group is $U(2)$, tadpole cancellation imposes the constraint that the numbers of vectors and anti-vectors of $U(2)$ should be equal. This constraint is discussed in [98], and lead in that context to a quantization of the number of families in multiples of three. This is not the case here, because the Higgs also makes contributions to the $U(2)$ anomaly. In the hidden sector more interesting examples of anomaly 
cancellation are possible, because chiral tensors may (and indeed do) contribute. These anomaly cancellations are of course a useful check on our results, but they are still far less restrictive than in six dimensions, where we have performed such checks on the complete solution to the tadpole solutions a few years ago.

The surviving part of the anomaly polynomial,

$$
\mathcal{P}(F)_{\mathrm{rest}}=\sum_{i} w_{i} \sum_{a b} A_{a b}^{i}\left[\operatorname{Tr}_{a} F \operatorname{Tr}_{b} F^{2}+\operatorname{Tr}_{a} F^{2} \operatorname{Tr}_{b} F\right]
$$

must be cancelled by a generalized Green-Schwarz mechanism. This mechanism involves coupling between $\operatorname{RR} p$-forms $C_{(p)}$ and the $U\left(N_{a}\right)$ field strength $F_{a}$. For the oriented open string these couplings are

$$
\sum_{a} S_{a}=\sum_{a} \int_{M_{4}} C_{(2)}^{a} N_{a} F_{a}+C_{(0)}^{a} \operatorname{Tr}_{a} F^{2}
$$

where we decided to expand the RR fields in the highly degenerate basis spanned by the complete set of boundary labels $a$. Here $F_{a}$ is the $U(1)_{a}$ field strength and $\operatorname{Tr}_{a} F^{2}$ is a trace over the vector representation of $U\left(N_{a}\right)$. In a geometric language the branes wrap homology classes $\pi_{a}$ and we can choose a basis $\omega_{a}$ of 3 -forms such that $\int_{\pi_{a}} \omega_{b}=\delta_{a b}$. The $\mathrm{RR}$ forms are KK reductions along this basis. We have $\int_{C Y} \omega_{a} \wedge \omega_{b}=I_{a b}$, the chiral intersection matrix of branes $a$ and $b$. Poincare duality in this basis then reads

$$
\star \mathbf{d} C_{(2)}^{a}=\sum_{b} I_{a b} \mathbf{d} C_{(0)}^{b}
$$

From the couplings (4.17) we can easily read off the contribution to the $U(1)_{a} S U\left(N_{b}\right)^{2}$ mixed anomaly,

$$
N_{a} I_{a b}
$$

For unoriented strings the sectors $a$ and $a^{c}$ are identified and the couplings are encoded in

$$
\sum_{a}\left(S_{a}+S_{a^{c}}\right)=\sum_{a} \int_{M_{4}} N_{a}\left[C_{(2)}^{a}-C_{(2)}^{a^{c}}\right] F_{a}+\sum_{a} \int_{M_{4}}\left[C_{(0)}^{a}+C_{(0)}^{a^{c}}\right] \operatorname{Tr} F_{a}^{2}
$$

where we sum over pairs $\left(a, a^{c}\right)$. The GS-diagram is proportional

$$
N_{a}\left(I_{a b}+I_{a b^{c}}-I_{a^{c} b}-I_{a^{c} b}\right)
$$

which has the correct form to cancel the mixed chiral $U(1)_{a} S U\left(N_{b}\right)^{2}$ anomaly.

\subsubsection{Massless $U(1) \mathrm{s}$}

A nonzero coupling $C_{(2)} F$ generates an effective mass for the $U(1)$ gauge field. A linear combination

$$
\sum_{i} \theta_{i} F_{i}
$$


is massless if and only if

$$
\sum_{i} \theta_{i} N_{i}\left[C_{(2)}^{i}-C_{(2)}^{i^{c}}\right]=0
$$

This equation can have nontrivial solutions because the basis $a$ that we are using is highly non-degenerate. It is natural to expand this "brane-basis" $\left\{C_{(2)}^{a}\right\}$ in a complete, nondegenerate basis $\left\{C_{(2)}^{(m, J)}\right\}$ that is one-to-one to the Ishibashi labels $(m, J)$

$$
C_{(2)}^{a}=\sum_{(m, J), w_{m} \neq 0} R_{a(m, J)} C_{(2)}^{(m, J)}
$$

where $R$ are the boundary reflection coefficients. This equation has a well-known geometrical analogue, namely as an expansion of the basis $\omega_{a}$ in a homology basis. In a geometric orientifold the tadpole conditions are written in a convenient homology basis $\pi_{i}$ for $H^{3}(C Y)$. The RR 2-form in the brane basis can then be expanded as $C^{a}=\sum_{i} \pi_{a i} C^{i}$ where $C_{i}$ are the reductions of the ten-dimensional RR5-form along the $\pi_{i}$ and $\pi_{a i}$ are the wrapping numbers or charges of the brane. From the CFT tadpole condition it follows that the Ishibashi labels are the natural basis at the Gepner point and that the reflection coefficients are the "wrapping numbers". This is the motivation for (4.19). Then (4.18) becomes

$$
\sum_{i} \theta_{i} N_{i}\left[R_{a(m, J)}-R_{a^{c}(m, J)}\right]=0
$$

for all Ishibashi labels $(m, J)$.

\subsection{Results}

In this section we will present the results of our search. We list some statistics and characteristics of the Gepner models and MIPFs we scanned in tables 4.2, 4.3 and 4.4. Furthermore we display plots of distributions of standard model particle multiplicities, the Higgs and some characteristics of the various models like ratios of gauge couplings, the number of hidden branes and features of the hidden gauge group. We will also present a small investigation into varying the number of chiral families.

\subsubsection{The numbers}

In total we found 179520 distinct standard model spectra with solutions to all tadpole equations. There are few cases where the same spectra are obtained for different MIPFs. In some cases these MIPFs have the same Hodge numbers and are also otherwise indistinguishable. This occurs for example for the tensor product $(2,2,2,6,6)$ and presumably indicates an unresolved redundancy. In other cases, the Hodge numbers are different, although, surpsisingly, the open sector is identical. The total number of such equivalent spectra is however very small, and if we remove these equivalences there are 179119 left. In the rest of the paper we use the former set as the basis of our analysis. 
In table 4.2 we list for each Gepner model the search results. The second column contains the values of the factors $k$ in the tensor product. Models for which we only searched for solutions of type 0 and 1 are denoted by a dagger $\dagger$. The third column specifies the number of primaries, the fourth the number of simple currents and the fifth gives information on the modular invariant partition functions. In this column, the first entry is the total number of symmetric simple current MIPFs. This is computed after removing MIPFs that are related to each other by permutations of the identical factors of the tensor product. Generically, all MIPFs related to different simple current subgroups and different matrices $X$ are distinct. However, in special cases generically distinct simple current MIPFs coincide (the simples example is $S U(2)$ level 2, where the generic A and $\mathrm{D}$ invariants coincide). In the table we list the number after removing such coincidences. Between parentheses we indicate for how many of these MIPFs the tadpole conditions were not completely solved for all standard model brane stack configurations; however even in those cases we searched for all solutions with at most three extra branes (or at most two if the number of candidate branes was larger than 400). The next entry in column four is the number of MIPFs for which at least one standard model brane configuration was found, and the last entry in column 4 is the number of MIPFs for which a solution to the tadpole equations was obtained. Column five gives the total number of standard model configurations, summed over all MIPFs, column six gives the total number of configurations with solutions to the tadpole conditions, and the last column gives the total number of distinct (as defined in section 4.1.3) standard model spectra.

In total we found solutions to the tadpole equations for 44 of the 168 Gepner Models and for 333 of the 5403 MIPFs. For 4079 MIPFs we did not even find any standard model four stack configuration. In 649 cases there are four-stacks, but no solution to the tadpole equations and in 342 cases we could not rule out the existence of solutions. The total number of standard model configurations that exist is 45051902 ; of these 1635985 yield solutions. Many of the latter have the same standard model spectrum, which reduces the total to 179520 .

The 168 models are listed in a particular order, starting with the ones with the smallest number of factors. More or less coincidentally this order corresponds rather well to the degree of difficulty, in decreasing order. The number of $(a, b, c, d)$ stacks is very small at the bottom of the list, and increases to millions at the top, but only for particular tensor combinations. However, despite the large number of candidates, the tensor combinations at the top of the list yield very few solutions to the tadpole equations. Some tensor combinations with a large number of solutions have a recognizable feature in common: the values of $k+2$ typically takes values that factorize into powers of 2 and 3 . However, there are counterexamples in both directions.

In table 4.3 we list the 333 modular invariants for which tadpole solutions were found. Column two specifies the MIPF in terms of the Hodge numbers of the corresponding type-IIB Calabi-Yau compactification ${ }^{4}$ and the number of singlets in a Heterotic compact-

\footnotetext{
${ }^{4}$ Hence $h_{11}$ is the number of hyper multiplets (not including the universal one from the gravitational sector) and $h_{21}$ the number of vector multiplets of the closed type IIB string before orientifolding.
} 
ification. Unfortunately this does not always specify the MIPF uniquely. However, in most cases they are distinguished by the number of boundaries, listed in column three. Column 4 contains the (unique) label of the MIPF5. In the last column we list the number of different Standard model spectra organized according to type. The first six entries refer to types $0, \ldots, 5$ defined above, with a massless $B-L$ vector boson. The last entry is the number of type 0 spectra with a massive $B-L$. In principle this could have occurred for type 1 as well, but no examples were found.

Table 4.4 lists the total number of solutions for each type, where we distinguish chiral subtypes for types 1,3 , and 5 . These subtypes are defined in terms of the contribution of the quark doublets, the lepton doublets and the Higgses to the $U(2)_{\mathrm{b}}$ anomaly. Since this anomaly cancels, the subtypes are characterized by two independent parameters. It is easy to see that the quark contribution must be an odd multiple of three (which we have chosen to be positive), the lepton contribution must be odd, and the Higgs contribution even. If the lepton contribution is larger than three the spectrum contains "chiral mirror leptons", i.e. mirror pairs of leptons that are chiral with respect to the full $\mathrm{CP}$ group, but non-chiral with respect to the standard model gauge group. For example, to get a lepton $S U(2)$ anomaly of -5 one must have the representation $4\left(1,2^{*},-\frac{1}{2}\right)+\left(1,2^{*}, \frac{1}{2}\right)$ (up to purely nonchiral mirrors). Strictly speaking such models can perhaps not be described as "just the chiral standard model", but we admitted them as a curiosity. The same phenomenon is possible for quarks as well, but we did not find any examples. Note that the last column shows twice the number of chiral supersymmetric Higgs pairs $\left(1,2, \frac{1}{2}\right)+\left(1,2,-\frac{1}{2}\right)$, so that the number of such Higgs pairs in the entire set can be $0,1,2,3,4$ or 6 . Interestingly only 0 and 3 were found for type 1 .

\subsubsection{Features of found spectra}

As discussed above there are several features we have considered as relevant to distinguish standard model spectra. We will now present which values these parameters take and see if there is any notable structure.

Looking at these distributions one could be tempted to draw statistical conclusions. Even if one would adopt this point of view, there are several reasons why one should be careful. For one, our search algorithm was set up to maximize the number of different solutions (see section 4.1.3). Also there are relations between different distributions (for example if stack c is realized as $S O(2)_{\mathrm{c}}, e^{c}$ and $\nu^{c}$ come from the same branes, hence have the same mirror-distribution). Another bias to keep in mind is caused by the fact that equations are much easier to solve for a small number of branes. This biases the search towards fewer branes.

First we will look at the number of mirrors of the standard model particles. The total chirality of the standard model particles is fixed to 3 . We allowed however for additional non-chiral pairs, the so-called mirrors. The plot for the number of mirrors is similar for

\footnotetext{
${ }^{5}$ This label specifies a simple current subgroup and a matrix $X$ that defines the modular invariant. It is difficult to list all this information efficiently, but it is available from the authors on request.
} 
all standard model particle hence we show only the one $(e, \nu)$ mirror pairs and the total number of mirrors of standard model particles in a model. It is interesting to see that the

Figure 4.1: Mirrors
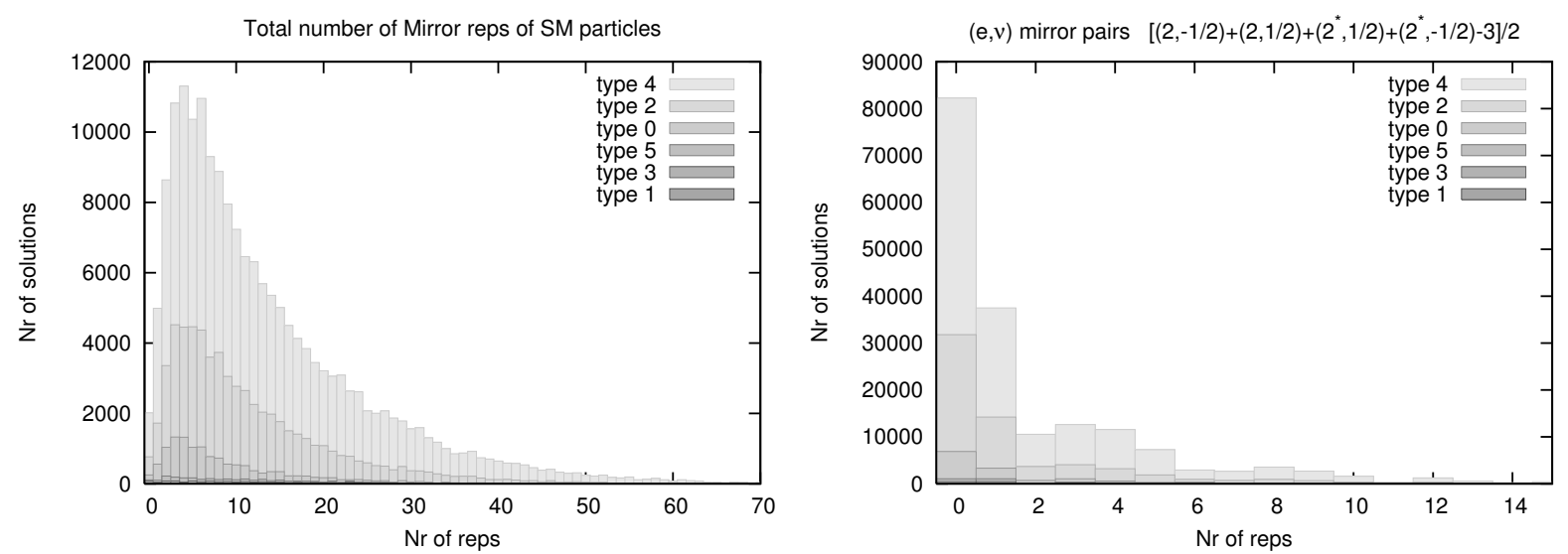

distribution of number of mirrors is sharply peeked at zero mirrors. From the total plot we see that 2018 models have no mirrors at all and that the distribution peeks at 4 mirrors.

The only bifundamentals coming from string states between the standard model branes which should not be chiral are strings stretched between branes a and d. These particles would be leptoquarks. In figure 4.5.2 we plot the number of non-chiral leptoquarks. The

Figure 4.2: Non-Chiral Leptoquarks
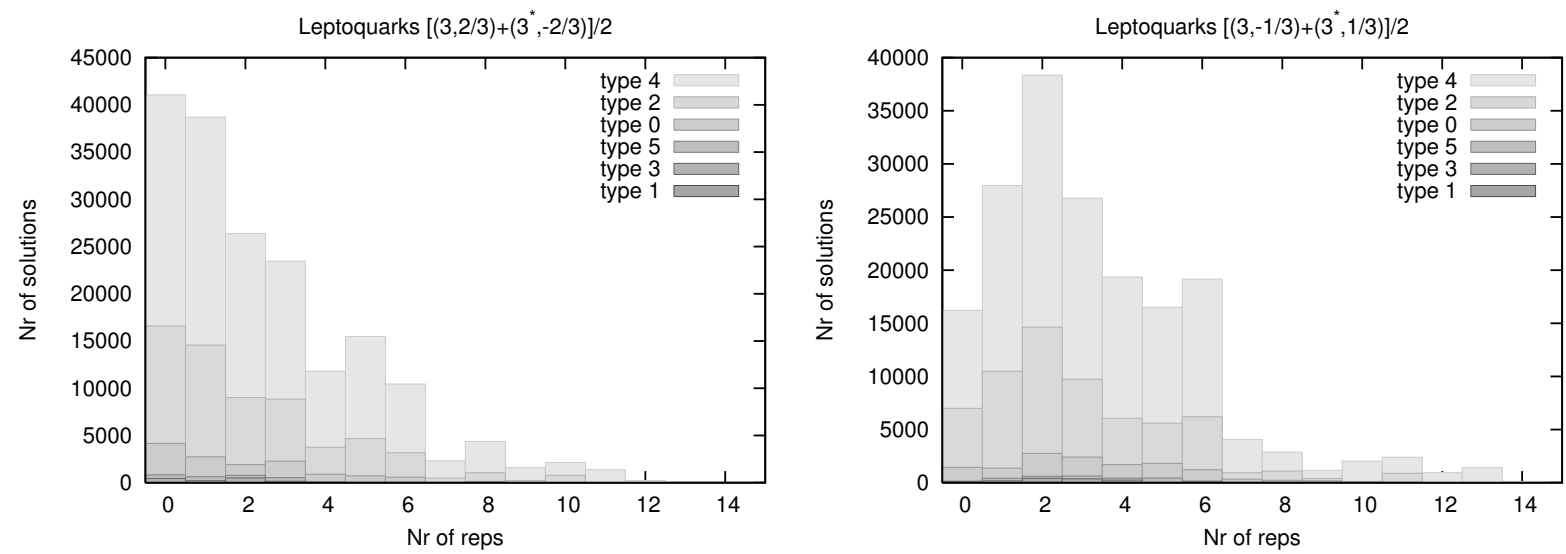

distribution of leptoquarks with equal sign lepton and baryon number is peaked at zero. This is the only non-chiral 'exotic' that peaks at zero (apart from individual mirror distributions, as noted above). The distribution of the opposite sign leptoquarks distribution peaks at 2 .

Finally one can have massless non-chiral representations coming from strings that have both ends on the same SM brane (or one end on the conjugate brane). These states are symmetric, anti-symmetric or adjoint tensors of the standard model gauge group. The 
distributions of non-chiral pairs of all these particles peak at a non-zero value, except for the ones where in a majority of the models that particular state does not exist. In 4.5.2 we plot the total number of these particles in a model and the distribution of adjoints of $S U(3)$ as an example.

Figure 4.3: Non-Chiral tensor representations
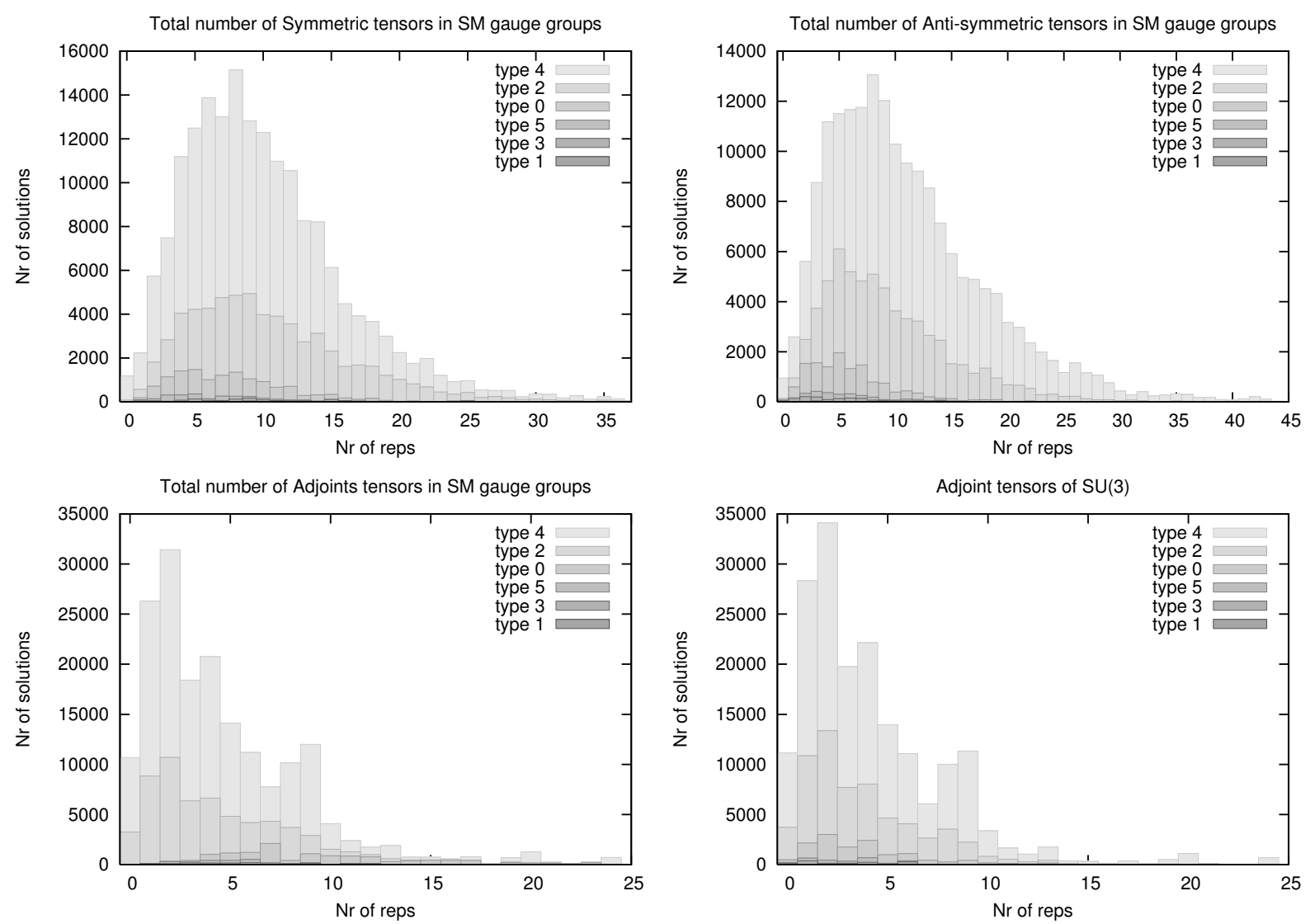

\subsubsection{Higgs}

In supersymmetric extensions of the standard model the Higgs always comes in non-chiral (w.r.t. to $G_{S M}$ ) pairs so the Higgsinos will not generate an Y-anomaly. In figure 4.5.3 we plot the number of Higgsino pairs, which is thus equal to half the number of standard model Higgs doublets in that model. As is clear from the picture, the number of Higgs pairs peaks at three. The maximum number of Higgs pairs we found is 56. Note that there also models with no Higgs. These models have an obvious deficiency, although it is

\footnotetext{
${ }^{6}$ Adjoints of $S O(2)$ are counted as anti-symmetric tensors, adjoints of $S p(2)$ as symmetric tensors. Massless anti-symmetric representations of $U(1)$ actually have no massless states at all, and were not counted.
} 
Figure 4.4: Number of Higgs

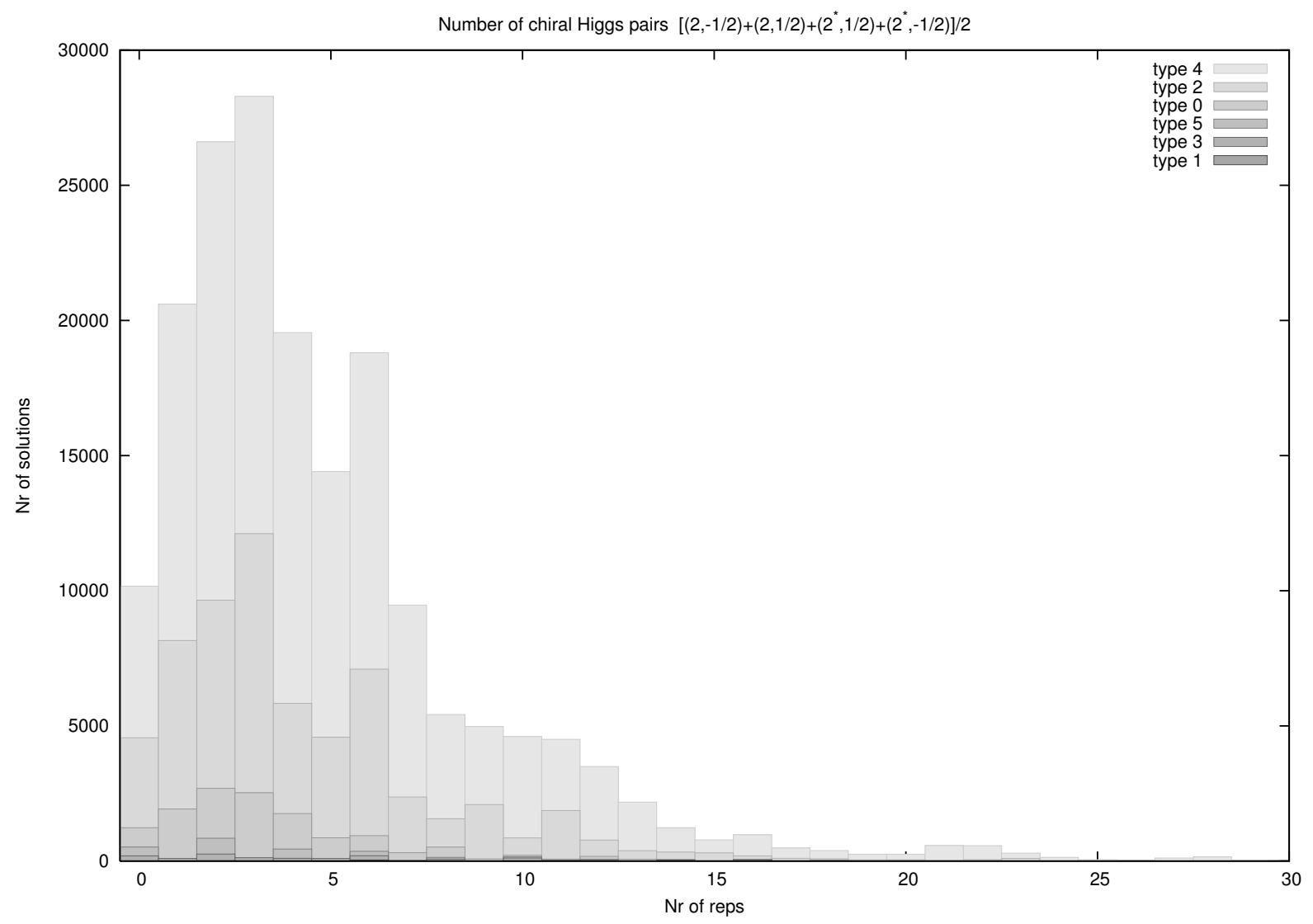

conceivable that some (composite-)particle from the hidden sector will play the rôle of the Higgs.

For types based on $U(2)_{\mathrm{b}}$ (type 1,3 and 5) there is a possibility for the Higgs of being chiral with respect to $U(2)_{\mathrm{b}}$. This chirality was ignored in figure 4.5.3, but the chirality distribution is display in 4.4 , as explained above.

\subsubsection{Hidden branes}

Our search algorithm was set up to maximize the different standard model spectra, not the hidden brane degrees of freedom. Hence we present in all plots and tables the number of solutions where we identified solutions with different hidden sectors, but which are otherwise equivalent. As explained in section 4.1.3 we did however construct all solutions containing 0,1 and 2 extra branes for all standard model brane stacks, three extra branes for all stacks with less than 400 candidate hidden CP groups, and four extra branes for less than 100 candidate hidden CP groups. In many other cases we attempted to extract a solution from the tadpole equations without a limit on the number of branes. The latter searches were stopped as soon a one solution was found and are limited by computer time constraints. Therefore they are not systematic. In figure 4.5.4 we show the total number of 
solutions found for each hidden brane multiplicity. This plot is based on a total of 10526078 solutions. These solutions are different only in the sense that their CP multiplicities are distinct. Undoubtedly there are still some equivalences in this set that were not taken into account. The number of solutions with 0,1 and 2 branes is 31215,148324 and 1170556 respectively. As one can see, the number of solutions grows very fast with the number of

Figure 4.5: Hidden sector of all solutions
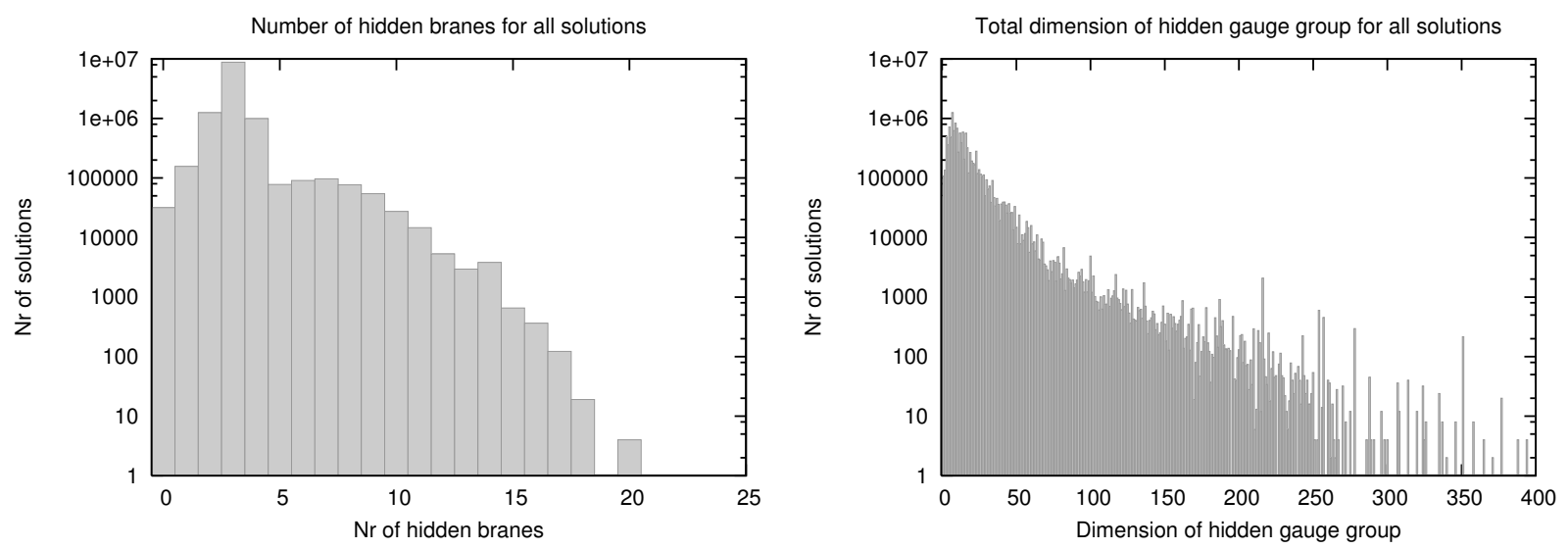

hidden branes, but is cut off beyond three branes for the reasons explained above. One can make a plausible guess what the picture might really look like, had we been able to push the search for solutions much further. Most likely, it would continue growing dramatically for quite a while. Since the number of candidate CP groups is typically a few hundred, the distribution necessarily peaks well below that number, but this could easily be around ten or twenty. It is clear that the total number of solutions with distinct standard model plus hidden spectrum can be many orders of magnitude larger than those we found. In figure 4.5 we plot the total dimension of the total hidden gauge group. The biggest gauge group we encountered has $\operatorname{dim} 780$. The biggest factors we found are $S O(18), S p(24)$ and $U(18)$.

\subsubsection{Gauge couplings}

A remarkable property of the standard model is that its gauge group fits naturally within the gauge group $S U(5)$. This explains two empirical facts: the observed quantization of electric charge, and the observed convergence of the coupling constants at short distances (although with present data, the latter success only survives if the additional assumption of supersymmetry is made). Both of these nice features seem to be lost in the class of models considered here.

Fractional charges are inevitable if there exist additional branes outside the standard model. Strings stretching between the standard model and the hidden branes yield particles (fundamental or QCD bound states) with half-integer electric charge. Although they may be non-chiral (as they are in all models presented here) or even completely absent from the massless spectrum, they exist inevitably as massive open string states. Interestingly, it is 
not completely straightforward to realize group-theoretical unification in heterotic strings either. In the standard realizations with Kac-Moody level 1 one ether gets $S U(5)$ without a massless Higgs boson to break it, or one gets $S U(3) \times S U(2) \times U(1)$ with additional (though not necessarily massless) fractional charges [127]. These problems can be avoided, for example by considering higher Kac-Moody levels, but it is difficult to argue that charge quantization is a natural property of string theory.

Heterotic $S U(5)$ and $S O(10)$ models do explain the observed coupling constant convergence, but they make a troublesome prediction for the unification scale, which is off by two to three orders of magnitude. It is on this point that open string models have an advantage, simply because there is no such prediction [138]. But there is also no prediction for the unification of the gauge couplings, because they emerge from dilaton couplings of four, a priori unrelated, branes.

In [33] it is argued that a realization of a supersymmetric extension of the standard model with intersecting branes naturally leads to a model where respectively branes $a$ and $d$ and $c$ and $b$ wrap the same cycles. This leads then at the string scale to the following relation between the three standard model coupling constants:

$$
\frac{1}{\alpha_{Y}}=\frac{2}{3} \frac{1}{\alpha_{s}}+\frac{1}{\alpha_{w}}
$$

That would mean that these models do not necessarily have full unification, but they do reproduce a relation which is compatible with the $S U(5)$ relation between the coupling constants.

To do a proper calculation of gauge unification we would have to assume a unification scale and evolve the couplings downward from that scale. Since we are in a rational point of the moduli space, this scale is fixed to a value of order the string scale. The massless spectrum we obtain contains, in general, several non-chiral particles that are presumably artifacts of the rational points. If one assumes that all these particles remain massless until the $\mathrm{TeV}$ scale, one should take them into account in the renormalization group flow. More ambitiously, one could work out the masses and the gauge couplings as a function of the moduli near the rational point, and take all of this into account. Here we will only address a simpler question, namely if there is any evidence for relations among the couplings at the string scale. If there is no relation, then clearly one might still get the correct low energy gauge couplings by starting outside the unification point in the space of couplings, and compensate this with exotic matter in the renormalization group flow, but the concept of unification is then anyway lost.

The coupling constants can be computed as follows. Up to a universal factor, they are given by

$$
\frac{1}{g_{\mathrm{a}}^{2}}=R_{0 \mathrm{a}} e^{\phi}
$$

for CP-groups of orthogonal and symplectic type and

$$
\frac{1}{g_{\mathrm{a}}^{2}}=\left(R_{0 \mathrm{a}}+R_{0 a^{c}}\right) e^{\phi}=2 R_{0 \mathrm{a}} e^{\phi}
$$


for unitary groups, with the conventional normalization $\operatorname{Tr} \mathrm{T}^{\mathrm{a}} \mathrm{T}^{\mathrm{b}}=\frac{1}{2} \delta^{\mathrm{ab}}$ for the generators in all three cases. This immediately gives the following expression for the ratio of weak and string couplings:

$$
\frac{g_{2}^{2}}{g_{3}^{2}}=\frac{R_{0 \mathrm{a}}}{\kappa R_{0 \mathrm{~b}}}
$$

where $\kappa=1$ for spectra of types 1,3 and 5 , and $\kappa=\frac{1}{2}$ for spectra of type 0,2 and 4 . The canonically normalized $U(1)$ generator of stack a is $Y_{\mathrm{a}}=\frac{1}{\sqrt{6}} \mathbf{1}$, for stack d it is $Y_{\mathrm{d}}=\frac{1}{\sqrt{2}} \mathbf{1}$, and for stack c it is $Y_{\mathrm{c}}=\frac{1}{\sqrt{2}} \mathbf{1}$ for complex boundaries, and $Y_{\mathrm{c}}=\frac{1}{2} \sigma_{3}$ for real ones. The standard model $U(1)$ factor $Y$ is the given by

$$
\frac{1}{\sqrt{6}} Y_{\mathrm{a}}-\frac{1}{\sqrt{2}} Y_{\mathrm{c}}-\frac{1}{\sqrt{2}} Y_{\mathrm{d}}
$$

for types 0 and 1 , and

$$
\frac{1}{\sqrt{6}} Y_{\mathrm{a}}-Y_{\mathrm{c}}-\frac{1}{\sqrt{2}} Y_{\mathrm{d}}
$$

for types 2,3,4 and 5. This leads to the following expression for the coupling constants

$$
\frac{1}{g_{Y}^{2}}=\frac{1}{6} \frac{1}{g_{\mathrm{a}}^{2}}+\frac{1}{2} \frac{1}{g_{\mathrm{c}}^{2}}+\frac{1}{2} \frac{1}{g_{\mathrm{d}}^{2}}
$$

for types 0 and 1 , and

$$
\frac{1}{g_{Y}^{2}}=\frac{1}{6} \frac{1}{g_{\mathrm{a}}^{2}}+\frac{1}{g_{\mathrm{c}}^{2}}+\frac{1}{2} \frac{1}{g_{\mathrm{d}}^{2}}
$$

for types 2,3,4 and 5. Hence in both cases the expression for the couplings in terms of reflection coefficients is the same:

$$
\frac{1}{g_{Y}^{2}}=\left(\frac{1}{3} R_{0 \mathrm{a}}+R_{0 \mathrm{c}}+R_{0 \mathrm{~d}}\right)
$$

The fact that the same expression is obtained is due to the fact that an orthogonal or symplectic c-stack can be viewed as a limit of a unitary one, with the two conjugate branes moved on top of the orientifold plane. From this expression we obtain the following formula for $\sin ^{2} \theta_{W}=g_{Y}^{2} /\left(g_{2}^{2}+g_{Y}^{2}\right)$ :

$$
\sin ^{2} \theta_{W}=\frac{\kappa R_{0 \mathrm{~b}}}{\kappa R_{0 \mathrm{~b}}+\frac{1}{6} R_{0 \mathrm{a}}+\frac{1}{2} R_{0 \mathrm{c}}+\frac{1}{2} R_{0 \mathrm{~d}}}
$$

In figure 4.5.5 we plot $\sin ^{2}\left(\theta_{w}\right)$ against the ratio $\alpha_{s} / \alpha_{w}$. In this plot the value for $S U(5)$ unification are also indicated, as well as its renormalization group flow. The solid line denotes the upper limit on $\sin ^{2}\left(\theta_{w}\right)$. Clearly the models we found occupy a substantial part of the allowed region. It is amusing to see that this point falls neatly in the middle of the cloud formed by our models. One aspect of the plot that attracts attention is a 
Figure 4.6: Gauge coupling constants at the string scale

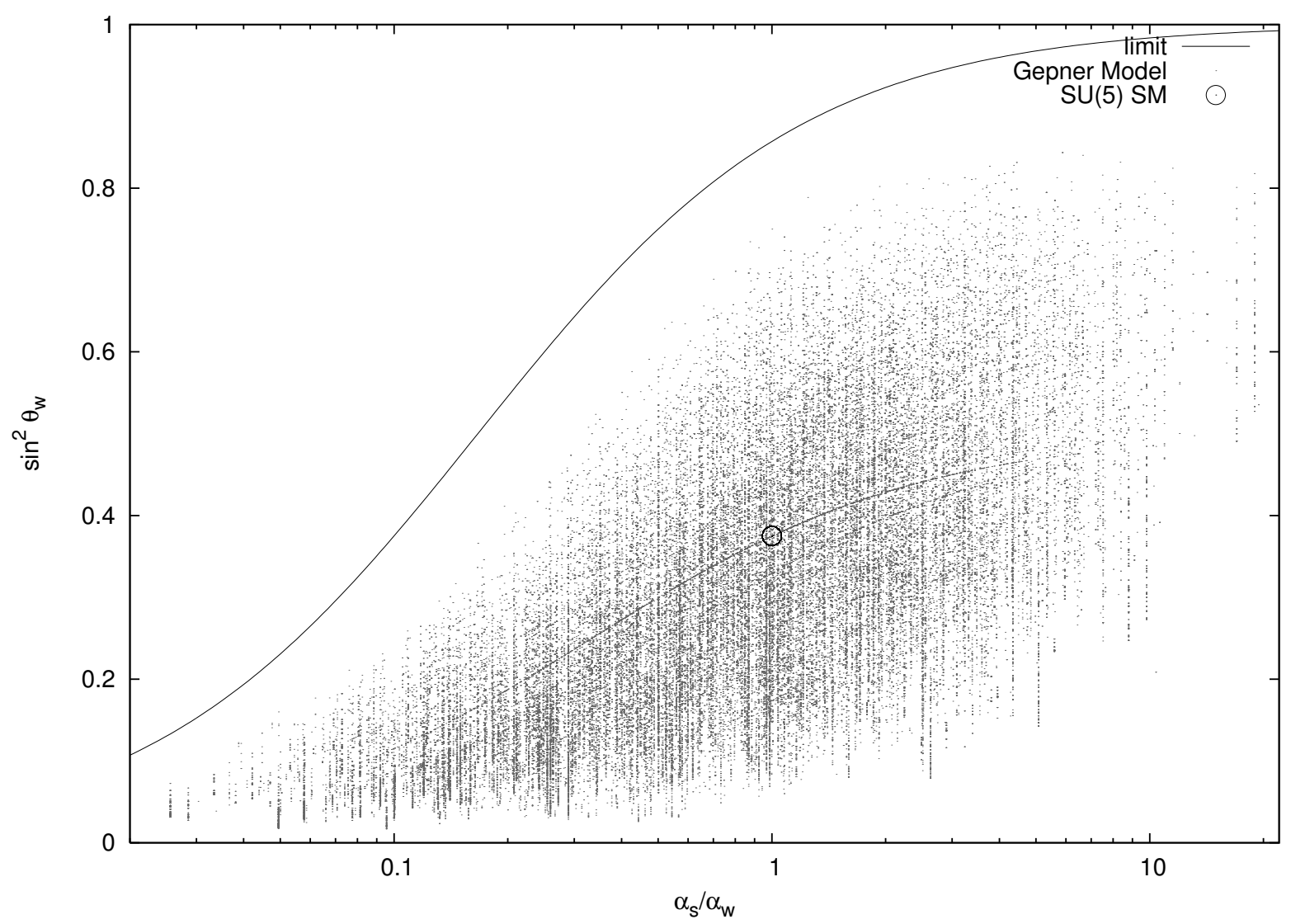

faint line ${ }^{7}$ roughly in the middle of the cloud. It turns out to be described by relation (4.20). Approximately $10 \%$ of the models is on that line. Not surprisingly, these models are characterized by the fact that the reflection coefficients for respectively branes $a$ and $d$ and branes $b$ and $c$ are the same, which is precisely the RCFT equivalent of the condition that [33] used for unification. So although a portion of the models we found has a relation between the coupling constants compatible with $S U(5)$ unification, this is certainly not a generic feature.

\subsubsection{Varying number of chiral families}

For a limited set of models we repeated our search for the standard model spectrum with a different number of families, in order to get a feeling what the constraint of having 3 chiral families means. Because more chiral particles implies more intersections one could expect that getting a spectrum with more chiral families is harder. Indeed this tendency is visible in plots 4.5.6. Because the number of standard model brane configurations to be

\footnotetext{
${ }^{7}$ This line is not very visible in the picture. A picture with higher resolution can be downloaded from http://www.nikhef.nl/ t58/GaugeCouplings.ps
} 
analyzed for 1 and 2 families was so big, we performed a search for 1 up to 9 families for 18 models and a search for 3 up to 9 models for a bigger set of 78 models.

But there is more structure. For example odd types (the ones based on $U(2)_{\mathrm{b}}$ ) are much more abundant with even families. If one takes a closer look and distinguishes by subtype, that is the way the $U(2)_{\mathrm{b}}$ anomaly cancels, this becomes a bit clearer. Both states coming from $[b, x]$ and $\left[b^{*}, x\right]$ can contribute to the total chirality of the $S U(2)_{W}$ doublets. They do however contribute oppositely to the $U(2)$ anomaly. This leaves the possibility to cancel the anomaly between the different $U(2)$ chiralities of a doublet. In the even family number models solutions of the subtype where the anomaly cancels fully between the different $U(2)$ chiralities of a given doublet are approximately three orders of magnitude more abundant than subtypes where the anomaly cancels partly between different doublets. Apparently it is easy to have the intersection number of $[b, x]$ equal to that of $\left[b^{*}, x\right]$. This is also explains why there are so relatively few odd family number solutions with $U(2)_{\mathrm{b}}$; For the family number to add up to an odd number, one of the two intersection numbers $[b, x]$ and $\left[b^{*}, x\right]$ has to be odd and the other even. The easy way of cancelling the anomaly is not a possibility.

Under the assumption that more chiral intersections are harder to get, we can also understand why 4, 2, 3 and 5 are typically less abundant then types 0 and 1 . The latter types are based on $U(1)_{c}$ and have all weak singlets coming from their own intersection, with $c$ or $c^{c}$. The former types however, have a real $c$-brane and both lepton singlets (and both quark singlets) come from the same intersection. This means that they have 2 times the number of families less chiral intersections.

A similar effect does not occur if we exchange $U(2)_{\mathrm{b}}$ for $S p(2)_{\mathrm{b}}$, because as discussed above, both $U(2)_{\mathrm{b}}$ chiralities contribute to one doublet. Hence both types based on $U(2)_{\mathrm{b}}$ and $S p(2)_{\mathrm{b}}$ have the same number of chiral $b$-brane intersections.

Also in the other types there is a clear even/odd effect. But if one looks, for odd/even family number separately, at the trend in the number of solutions for a certain type against family number it is to good approximation an exponential drop.

One feature that deserves mentioning is the occurrence of type 1 models with a massive $B-L$ for 1,2 and 4 families, something we did not find for three families. These models have as the gauge group that couples to the standard model particles precisely the standard model. That this did not occur for three families is probably a matter of statistics. As we have seen type 1 models with three families are already rare, and furthermore from the search for 1,2 and 4 family number models we know that a massive $B-L$ only happens in a few percent of the type 1 solutions.

\subsection{The simplest case}

Here we will discuss in some detail one of the simplest cases where a solution was found. It occurs for the tensor product $(1,1,1,1,7,16)$. This has 1944 primaries, 81 of which are simple currents forming a discrete group $\mathbb{Z}_{3} \times \mathbb{Z}_{3} \times \mathbb{Z}_{3} \times \mathbb{Z}_{3}$. Since there are only odd factors, there is just one orientifold choice for each MIPF. 
After removing all permutations of the identical factors, this tensor product has 34 simple currents MIPFs. Only one of them has standard-model-like (a,b,c,d) branes. This MIPF is defined by a simple current group that is isomorphic to $\mathbb{Z}_{3} \times \mathbb{Z}_{3} \times \mathbb{Z}_{3}$, and is generated by the three currents:

$$
\begin{gathered}
{[0,0,0,0,(1,-1,0),(7,3,0), 0]} \\
{[0,0,0,0,(1,-1,0),(7,3,0), 0]} \\
{[0,0,(1,-1,0), 0,0,(7,-5,0),(16,16,0)] .}
\end{gathered}
$$

Our notation is as follows: between the square brackets we indicate the decomposition of the tensor product representation into minimal model representations, with the first entry in each square bracket representing the space-time sector. The minimal model representations are indicated in the usual way as $(l, q, s)$, except for the vacuum, which is denoted as " 0 ". Note that only orbit representatives are shown: The minimal model representations are subject to field identification, and the entire tensor representation is actually just one representative of a chiral algebra orbit. Hence other, but equivalent, expressions can be given.

The matrix $X$ yielding the MIPF under consideration is

$$
\frac{1}{3}\left(\begin{array}{lll}
1 & 0 & 1 \\
0 & 1 & 1 \\
1 & 1 & 1
\end{array}\right)
$$

This yields a closed string spectrum corresponding to Hodge numbers $(23,23)$, and a heterotic spectrum with 217 singlets. There are 72 Ishibashi states and hence the same number of boundary states. The total number of sets of (a,b,c,d) stacks is six, all of them of type 3. Three sets saturate the dilaton tadpole condition, whereas the other three do not. We find that for the former all other 17 tadpole conditions are also satisfied, whereas for the latter three there is no possibility of adding branes in order to solve the tadpole conditions. The three sets of boundaries yielding solutions can be described as follows

$$
\begin{array}{ll}
\text { set } 1: & \left(a_{1}, a_{1}^{c}\right)\left(b, b^{c}\right)(c)\left(d, d^{c}\right) \\
\text { set } 2: & \left(a_{2}, a_{2}^{c}\right)\left(b, b^{c}\right)(c)\left(d, d^{c}\right) \\
\text { set } 3: & \left(a_{3}, a_{3}^{c}\right)\left(b, b^{c}\right)(c)\left(d, d^{c}\right)
\end{array}
$$

in other words, the b,c and d branes are always identical, and the complete set of boundaries 
we need is given by

$$
\begin{aligned}
& \mathrm{a}_{1}:[0,0,0,0,0,(1,-1,0),(2,2,0)] \\
& \mathrm{a}_{1}^{c}:[0,0,0,0,0,(1,1,0),(2,-2,0)] \\
& \mathrm{a}_{2}:[0,0,0,0,0,(1,-1,0),(8,2,0)]_{1} \\
& \mathrm{a}_{2}^{c}:[0,0,0,0,0,(6,4,0),(8,-8,0)]_{1} \\
& \mathrm{a}_{3}^{c}:[0,0,0,0,0,(1,-1,0),(8,2,0)]_{2} \\
& \mathrm{a}_{3}^{c}:[0,0,0,0,0,(6,4,0),(8,-8,0)]_{2} \\
& \mathrm{~b}:[0,(1,-1,0),(1,-1,0),(1,-1,0),(1,-1,0),(6,-6,0), 0] \\
& \mathrm{b}^{c}:[0,(1,1,0),(1,1,0),(1,1,0),(1,1,0), 60,0] \\
& \mathrm{c}:[0,0,0,0,(1,1,0),(7,7,0),(16,16,0)] \\
& \text { d }:[0,0,0,0,0,(7,7,0),(16,-14,0)] \\
& d^{c}:[0,0,0,0,0,(7,-7,0),(16,14,0)]
\end{aligned}
$$

The three sets only differ in the choice of the $U(3)$-brane. The last two choices, $\mathrm{a}_{2}$ and $\mathrm{a}_{3}$ and their boundary conjugates, are resolved fixed points of the supercurrent extension of the tensor product., and they differ only in their fixed point degeneracy labels, indicated here as 1 and 2. Not surprisingly, they yield the same spectrum. The spectra of sets 1 and 2 are distinct, and are as follows

$$
\begin{gathered}
5\left(V, V^{*}, 0,0\right)_{3}+9(V, 0, V, 0)_{-3}+ \\
5(0, V, V, 0)_{3}+3(0, V, 0, V)_{3}+ \\
3(0,0, V, V)_{-3}+12(V, V, 0,0)_{0}+ \\
4\left(0, V, 0, V^{*}\right)_{0}+8(A d, 0,0,0)_{0}+ \\
4(A, 0,0,0)_{0}+8(S, 0,0,0)_{0}+ \\
4(0, A, 0,0)_{0}+3(0,0, S, 0)_{0}+ \\
4(0,0,0, A)_{0}
\end{gathered}
$$

and

$$
\begin{gathered}
3\left(V, V^{*}, 0,0\right)_{3}+9(V, 0, V, 0)_{-3}+ \\
5(0, V, V, 0)_{3}+3(0, V, 0, V)_{3}+ \\
3(0,0, V, V)_{-3}+4(V, V, 0,0)_{0}+ \\
4\left(0, V, 0, V^{*}\right)_{0}+4(A d, 0,0,0)_{0}+ \\
4(A, 0,0,0)_{0}+6(S, 0,0,0)_{0}+ \\
4(0, A, 0,0)_{0}+3(0,0, S, 0)_{0}+ \\
4(0,0,0, A)_{0}
\end{gathered}
$$


The notation is as follows: $V$ denotes a vector, $A$ and anti-symmetric tensor, $S$ a symmetric tensor and $A d$ and adjoint representation. The $*$ denote complex conjugation, and the last subscript denotes the net chirality of the representation. Our convention is to represent all four-dimensional fermions as left-handed spinors. The precise meaning of, for example, $N \times\left(V, V^{*}, 0,0\right)_{M}$ is then $\frac{1}{2}(N+M)\left(V, V^{*}, 0,0\right)+\frac{1}{2}(N-M)\left(V^{*}, V, 0,0\right)$. Hence in the first case, we have 3 chiral standard model $S U(3) \times S U(2) \times U(1)$ multiplets $\left(3,2, \frac{1}{6}\right)$, plus a single mirror pair $\left(3,2, \frac{1}{6}\right)+\left(3^{*}, 2,-\frac{1}{6}\right)$. Note that the second factor is actually $U(2)$ instead of $S U(2)$. The additional $U(1)$ is anomalous, and the corresponding gauge boson acquires a mass. The mirrors are non-chiral not just with respect to the standard model group, but with respect to the full group (although this was not a priori required). There is a second set of fully non-chiral mirrors of the same standard model representation in both cases, six in the first and 2 in the second. These differ from the former ones by having opposite $U(1)_{\mathrm{b}}$-charges. The tadpole equations satisfied by these two cases are in fact identical.

Since these spectra only differ by the choice of the $a$-branes, the lepton and Higgs sectors are identical. Both have 5 Higgs pairs $\left(1,2, \frac{1}{2}\right)+\left(1,2,-\frac{1}{2}\right)$, with the interesting feature that 3 of them are chiral with respect to the $U(2)$ group. Another noteworthy feature is the absence of lepto-quarks.

Finally we present the closed spectrum. The only model-dependent feature concerns the diagonal sector. It turns out that all relevant multiplicities are 1 , and that all nonvanishing Klein bottle coefficients are 1 . The 23 closed string vector multiplets originate from 21 diagonal fields in the closed string partition function, and two off-diagonal ones. Of the first 21 only the symmetric components survive the Klein bottle projection, and the two off-diagonal ones are reduced to a single field, consisting of a symmetric and an anti-symmetric component. Hence the projected closed string spectrum consists of a supergravity multiplet, one vector multiplet and 22 chiral multiplets originating from $h_{11}$ and another 23 chiral multiplets originating from $h_{21}$.

\subsection{Conclusions}

We performed the first semi-systematic search for standard model spectra in open string models. We presented 179520 models that have as their chiral spectrum just the standard model. Most of the models do have non-chiral exotics and/or mirrors, for which we presented the abundances. The number of Higgs was left a free parameter. We found models with as much as 56 Higgses. The distribution peaks at three pairs of Higgs. We also calculated the standard model gauge couplings and found (perhaps not surprisingly) no relation that hinted at unification.

Although we are confident that within the Gepner models we constructed most realizations of the "standard" four stack model, there is much room for improvement and generalization.

More MIPFs of the Gepner models are known than the ones considered here. First of all there are diagonal invariants and their simple current modifications. It turns out that in many cases (but not all) these can be obtained by simple currents, and hence are 
already included. In other cases more work is needed, see e.g. [26]. Furthermore there are exceptional $S U(2)$ invariants, exceptional invariants of $S U(2)$ tensor products [133], and invariants related to interchanges of fixed points. Unfortunately there is no boundary state formalism available to deal with these cases. There also is no guarantee that we found all orientifolds. Up to now, the orientifold degrees of freedom we use here (Klein bottle currents and crosscap signs) has included everything known by other methods, but most likely this is just a conjecture awaiting a counter example.

There are additional $N=2$ coset CFTs and presumably even RCFTs that are not cosets. Indeed, there is a the much larger class of Kazami-Suzuki [103] models. In principle our methods are applicable to these models as well, provided we know the exact spectrum. In cases without field identification fixed points this can be computed straightforwardly, but if there are fixed points the formalism has not been developed yet. Another class that might be accessible with similar methods are the interchange orbifolds of identical minimal or Kazami-Suzuki models [36, 18].

To get an idea how much is still missing, note that we encountered "only" 873 different Hodge number pairs, while for example in [111] already more than 30,000 are presented.

For any accessible RCFT one may consider other brane constructions than the four stack model, for example a construction that would yield $S U(5)$ unification at the string scale, or broken versions of such a model. This would have the obvious advantage of restoring gauge unification. One could also look for Pati-Salam like models, and for models that require a brane recombination mechanism to yield the standard model. As mentioned in the introduction, examples of these types have been constructed already. In principle there is no reason why the standard model could not be realized in such a way, but of course this opens a Pandora's box of possibilities. The number of solutions would explode even more if we were to allow chiral exotics, a possibility that can never be rigorously ruled out experimentally. But if indeed the quarks and leptons we know today provide misleading information about the true chiral spectrum of nature, then the goal of finding the standard model in string theory is essentially unachievable at present.

The obvious way to control this explosion of candidate standard models is to apply more constraints. There are several criteria that might be used to reject some of the many models on our list, such as absence of a Higgs candidate, the absence of plausible mechanisms to break $S p(2)_{\mathrm{c}}$, or to generate a $B-L$ gauge boson mass. However, it is hard to come up with a criterium that is at the same time very effective and unquestionable.

One could also try to work out the Yukawa coupling of the standard model particles to the Higgs sector. This requires open string three-point couplings, computable in principle in RCFT, but not yet in practice. In cases with more than one supersymmetric Higgs pair, there is the obvious problem of deciding which combination gets a vacuum expectation value. This could be treated as a set of free parameters, and fitted to the observed couplings. Doing that would requires a renormalization group evolution of those couplings, which in its turn requires detailed knowledge of the full (chiral and non-chiral) light spectrum. Then one still faces the problem of the moduli-dependence of the result. To some extent, this may be studied by probing some directions in moduli space with a large number of rational points, as we did for the gauge couplings. However, such an effort is probably premature. 
Appendix: Boundary and Ishibashi orbits

Most likely an essential ingredient is missing for the understanding of Yukawa couplings, and that is especially true for neutrino masses.

Fortunately more experimental constraints can be expected in the coming years, especially from the LHC, and also from neutrino and astrophysics experiments. With more experimental input we might come to the conclusion that another type of model should be searched for, and with a different set of constraints. It is to be expected that the class considered here will yield equally rich and abundant results in other cases.

\section{Acknowledgements:}

We would like to thank Dieter Lüst for suggesting a check of the results of [33]. We would like to thank Jeff Templon, David Groep, and Davide Salomoni for assistence with running jobs on the NIKHEF computer farm. The work of A.N.S. has been performed as part of the program FP 52 of the Foundation for Fundamental Research of Matter (FOM), and the work of T.P.T.D. and A.N.S. has been performed as part of the program FP 57 of FOM. The work A.N.S. has been partially supported by funding of the Spanish "Ministerio de Ciencia y Tecnología", Project BFM2002-03610. The work of L.R.H. wass supported in part by the Federal Office for Scientific, Technical and Cultural Affairs through the "Interuniversity Attraction Poles Programme - Belgian Science Policy" P5/27.

\section{Appendix: Boundary and Ishibashi orbits}

To compute all annuli using the formalism of [69] it is sufficient to compute all reflection coefficients, and use (4.6), summing over all Ishibashi labels to compute all annuli $A_{a b}^{i}$. Computationally this scales as $N_{i}\left(N_{B}\right)^{3}$, where $N_{i}$ is the number of primaries one needs to consider (in our case only the massless ones) and $N_{B}$ the number of boundaries, or equivalently, the number of Ishibashi labels. This sort of computation is analogous to the computation of the fusion coefficients using the Verlinde formula, which, for $N$ primaries, scales as $N^{4}$. In the latter case, such computations can be speeded up drastically if there is a non-trivial simple current group $\mathcal{G}$. This leads to relations among the matrix elements of the matrix $S$ of the form

$$
S_{J i, j}=e^{2 \pi i Q_{J}(j)} S_{i j}
$$

Fusion computations can then be made more efficient for the following three reasons

1. Simple current charge conservation, i.e.

$$
N_{a b c}=0 \text { if } Q_{J}(a)+Q_{J}(b)+Q_{J}(c) \neq 0 \bmod 1 .
$$

2. Simple current relations among fusion entries, $N_{K a, J b, L c}=N_{a b c}$ if $J L=K$

3. The summation in the Verlinde formula can be restricted to orbit representatives.

In the absence of fixed points, i.e if all orbit are equally long, this reduces the size of the computation by a factor $|\mathcal{G}|^{4}$ to $\left(N_{O}\right)^{4}$, where $N_{O}$ is the number of orbits. 
In the present case the quantity of interest is $A_{a b}^{i}$, for a subset of labels $i$. In order to make use of similar results, we have to establish an action of simple currents on the boundary labels $a$ as well as an action on the Ishibashi labels in the internal summation.

First we define a boundary simple current. Consider the oriented annulus

$$
A_{i a}{ }^{b}=\sum_{m} \frac{S_{i m}}{S_{0 m}} R_{m a} R_{m b}^{*}
$$

where for simplicity the boundary and Ishibashi labels $a$ and $m$ implicitly include the degeneracy label. The labels $i$ are primaries of the bulk CFT, and we can consider the case that $i$ is a simple current, $J$. Then one can show that for every $a$ there is precisely one $b$ such that $A_{J a} b=1$, and that for all other $b$ this quantity vanishes. The proof goes as follows.

$$
A_{J a}{ }^{b}=\sum_{m} \frac{S_{J m}}{S_{0 m}} R_{m a} R_{m b}^{*}=\sum_{m} e^{2 \pi i Q_{J}(m)} R_{m a} R_{m b}^{*}
$$

For each $a$, the vectors $R_{m a}$ have norm 1 . Hence this sum can at most be 1 , and it is equal to 1 if and only if

$$
R_{m b}=e^{2 \pi i Q_{J}(m)} R_{m a}
$$

Since the boundaries are independent, there can be at most one boundary $b$ that satisfies this. and since the set is complete, there must be precisely one (note that the matrix $A_{J}$ has to satisfy the completeness condition $A_{J} A_{J^{c}}=\mathbf{1}$ and hence cannot have an entire row that is zero). Hence it makes sense to define $J \cdot a \equiv b$ as the action of $J$ on $a$. Using (4.22) one can easily show that

$$
A_{i, J \cdot a}^{J \cdot b}=A_{i a}^{b} .
$$

This establishes the second of the three properties listed above, with $K=1$. Since the label $i$ is restricted to massless fields a further generalization analogous to $K \neq 1$ is not really useful, since the simple current action on $i$ does not preserve its conformal weight.

A simple current $J$ may yield a trivial action on all boundaries. It is easy to show that $J \cdot(K \cdot a)=(J K \cdot a)=K \cdot(J \cdot a)$, where $J K$ denotes the fusion product of the CFT. Therefore if $J$ and $K$ both fix all boundaries, so does $J K$, and hence the set of simple currents that fix all boundaries is a $\operatorname{subgroup} \mathcal{G}_{X}$ of $\mathcal{G}$. The boundary simple current group is defined as the discrete group $\mathcal{G} / \mathcal{G}_{X}$. The elements of that group have distinct actions on at least one of the boundaries.

In all cases covered by the simple current formalism of [69] there is a special boundary originating from the $\mathrm{CFT}$ vacuum state, $a=0$. One might conjecture a natural correspondence between the elements of the boundary simple current group and the set of boundaries obtained by the action of $\mathcal{G}$ on the boundary 0 . However, it turns out that this is not a one-to-one correspondence: it may happen that $J \cdot 0=0$, but $J \cdot a \neq a$ for some other $a$. Hence the boundary simple current group may be larger than the set of "simple current boundaries".

Relation (4.22) is the analog of (4.21) for the action on the boundary label. An action on the Ishibashi label can be derived directly from (4.9) and (4.11):

$$
R_{(I m, J)\left[a, \psi_{a}\right]}=F_{m}(I, J) e^{2 \pi i Q_{I}(a)} R_{(m, J)\left[a, \psi_{a}\right]},
$$


Appendix: Tables

if the set of degeneracy labels $J$ for $m$ and $L m$ are the same. This is the case if and only if $L$ is local with respect to the simple current group $\mathcal{H}$ that defines the MIPF. The subgroup of $\mathcal{G}$ with that property will be called the Ishibashi simple current group. The simple current twist $F_{m}(I, J)$ is a sign in all known cases, and this implies that its occurrence here is irrelevant, since we are free to change all boundary and crosscap coefficients simultaneously by signs: $R_{m a} \rightarrow \epsilon_{m} R_{m a}, U_{m} \rightarrow \epsilon_{m} U_{m}$. This allows us to redefine the boundary coefficients on each Ishibashi orbit in such a way that for the orbit representatives, $m_{0}$, we get simply

$$
R_{\left(m_{0}, J\right)\left[a, \psi_{a}\right]}=e^{2 \pi i Q_{I}(a)} R_{\left(m_{0}, J\right)\left[a, \psi_{a}\right]},
$$

Note that the crosscap coefficients $U_{m, J}$ vanish if $J \neq 0$, so these sign changes are irrelevant for crosscaps.

Now consider the first simplification listed above, charge conservation. The annulus amplitude $A_{a b}^{i}$ can be expressed in terms of the fixed point fusion coefficients [96]

$$
\left(N^{J}\right)_{a b}^{j}=\sum_{m} \frac{S_{j m}^{*}}{S_{0 m}} S_{m a}^{J} S_{m b}^{J^{c}} .
$$

as a linear combination of the form

$$
\sum_{L \in \mathcal{H}} \sum_{J \in c H} \phi(L, J, a, b)\left(N^{J}\right)_{a b}^{K L i}
$$

Here $\phi(L, J, a, b)$ are complex coefficients and $K$ is the Klein bottle current. Using (4.11) and the property $F_{a}(K, N) F_{a}(K, M)=F_{a}(K, N M)$ it follows that $\left(N^{J}\right)_{a b}^{j}=0$ unless $Q_{M}(a)+Q_{M}(b)=Q_{M}(j) \bmod 1$ for all simple currents $M$. For the annulus this implies that $A_{a b}^{i}=0$ unless there is an $L \in \mathcal{H}$ so that $Q_{M}(a)+Q_{M}(b) \neq Q_{M}(i)+Q_{M}(K)+Q_{M}(L)$ $\bmod 1$.

Finally consider the third simplification. The sum over $m$ can be reduced to a sum over Ishibashi orbit representatives provided that charge conservation is satisfied, i.e $Q_{I}(a)+$ $Q_{I}(b) \neq Q_{I}(i)+Q_{I}(K)+Q_{I}(L) \bmod 1$, for all $L \in \mathcal{H}$. Since Ishibashi currents $I$ are always local with respect to $\mathcal{H}$, this condition is always satisfied.

\section{Appendix: Tables}

Table 4.2: Summary results for all Gepner Models

\begin{tabular}{|l|c|c|c|c|c|c|c|}
\hline nr. & Tensor & Prim & S.C. & MIPF & Intersect. & Sol. & SM spectra \\
\hline 1 & $(1,5,41,1804)$ & 28539 & 1 & $1,0,0$ & 0 & 0 & 0 \\
2 & $(1,5,42,922)$ & 29772 & 2 & $2,-,-$ & - & - & - \\
3 & $(1,5,43,628)$ & 31482 & 3 & $2,-,-$ & - & - & - \\
4 & $(1,5,44,481)$ & 9399 & 1 & $1,0,0$ & 0 & 0 & 0 \\
5 & $(1,5,46,334) \dagger$ & 35442 & 6 & $4,1,0$ & 12 & 0 & 0 \\
6 & $(1,5,47,292) \dagger$ & 37800 & 7 & $2(1), 1,0$ & 1128 & 0 & 0 \\
7 & $(1,5,49,236)$ & 4575 & 1 & $1,0,0$ & 0 & 0 & 0 \\
\hline
\end{tabular}


Susy SM spectra from RCFT Orientifolds

Table 4.2: Summary results for all Gepner Models

\begin{tabular}{|c|c|c|c|c|c|c|c|}
\hline nr. & Tensor & Prim & S.C. & MIPF & Intersect. & Sol. & SM spectra \\
\hline 8 & $(1,5,52,187)$ & 12690 & 3 & $2(1), 1,0$ & 144 & 0 & 0 \\
\hline 9 & $(1,5,54,166) \dagger$ & 48258 & 14 & $4,1,0$ & 54 & 0 & 0 \\
\hline 10 & $(1,5,58,138)$ & 6156 & 2 & $2,0,0$ & 0 & 0 & 0 \\
\hline 11 & $(1,5,61,124)$ & 64449 & 21 & $4(2), 3,0$ & 81044 & 0 & 0 \\
\hline 12 & $(1,5,68,103)$ & 20748 & 7 & $2(1), 1,0$ & 234 & 0 & 0 \\
\hline 13 & $(1,5,76,89)$ & 2835 & 1 & $1,0,0$ & 0 & 0 & 0 \\
\hline 14 & $(1,5,82,82) \dagger$ & 108612 & 42 & $8(1), 3,0$ & 1744 & 0 & 0 \\
\hline 15 & $(1,6,23,598)$ & 12600 & 2 & $2,0,0$ & 0 & 0 & 0 \\
\hline 16 & $(1,6,24,310) \dagger$ & 13650 & 4 & $6(1), 1,0$ & 18260 & 0 & 0 \\
\hline 17 & $(1,6,25,214)$ & 14742 & 6 & $4(2), 2,0$ & 212000 & 0 & 0 \\
\hline 18 & $(1,6,26,166)$ & 15876 & 8 & $10(7), 8,0$ & 4939262 & 0 & 0 \\
\hline 19 & $(1,6,28,118)$ & 18270 & 12 & $12(9), 10,2$ & 1289765 & 217 & 67 \\
\hline 20 & $(1,6,30,94)$ & 20664 & 16 & $14(12), 12,1$ & 2912087 & 297 & 221 \\
\hline 21 & $(1,6,31,86)$ & 2464 & 2 & $2,0,0$ & 0 & 0 & 0 \\
\hline 22 & $(1,6,34,70)$ & 26460 & 24 & $20(16), 18,4$ & 1539069 & 2466 & 1587 \\
\hline 23 & $(1,6,38,58)$ & 8260 & 8 & $10(7), 8,1$ & 435815 & 36 & 18 \\
\hline 24 & $(1,6,40,54)$ & 4018 & 4 & $6(3), 3,0$ & 87494 & 0 & 0 \\
\hline 25 & $(1,6,46,46)$ & 46536 & 48 & $28(24), 26,5$ & 3687098 & 67723 & 16490 \\
\hline 26 & $(1,7,17,340)$ & 9396 & 3 & $2,0,0$ & 0 & 0 & 0 \\
\hline 27 & $(1,7,18,178)$ & 10224 & 6 & $4(1), 1,0$ & 29672 & 0 & 0 \\
\hline 28 & $(1,7,19,124)$ & 11880 & 9 & $6(2), 4,0$ & 2513 & 0 & 0 \\
\hline 29 & $(1,7,20,97)$ & 4116 & 3 & $2,0,0$ & 0 & 0 & 0 \\
\hline 30 & $(1,7,22,70)$ & 14760 & 18 & $12(6), 8,0$ & 38210 & 0 & 0 \\
\hline 31 & $(1,7,25,52)$ & 21060 & 27 & $10(2), 7,0$ & 7523 & 0 & 0 \\
\hline 32 & $(1,7,28,43)$ & 7128 & 9 & $6,2,0$ & 19 & 0 & 0 \\
\hline 33 & $(1,7,34,34)$ & 33264 & 54 & $16(8), 10,2$ & 62992 & 70 & 13 \\
\hline 34 & $(1,8,14,238)$ & 8082 & 4 & $6(3), 3,1$ & 3447270 & 16 & 16 \\
\hline 35 & $(1,8,16,88)$ & 10218 & 12 & $12(7), 7,0$ & 70962 & 0 & 0 \\
\hline 36 & $(1,8,18,58)$ & 12690 & 20 & $12(7), 9,1$ & 240804 & 4 & 2 \\
\hline 37 & $(1,8,22,38)$ & 2034 & 4 & $6(1), 1,0$ & 2888 & 0 & 0 \\
\hline 38 & $(1,8,28,28)$ & 28410 & 60 & $20(8), 14,2$ & 152706 & 1288 & 188 \\
\hline 39 & $(1,9,12,229)$ & 2875 & 1 & $1,0,0$ & 0 & 0 & 0 \\
\hline 40 & $(1,9,13,108)$ & 1015 & 1 & $1,0,0$ & 0 & 0 & 0 \\
\hline 41 & $(1,9,20,31)$ & 6160 & 11 & $2,0,0$ & 0 & 0 & 0 \\
\hline 42 & $(1,10,11,154)$ & 7704 & 6 & $4,1,0$ & 372 & 0 & 0 \\
\hline 43 & $(1,10,12,82)$ & 8970 & 12 & $12(4), 9,0$ & 277382 & 0 & 0 \\
\hline 44 & $(1,10,13,58)$ & 10332 & 18 & $12(4), 9,0$ & 19649 & 0 & 0 \\
\hline 45 & $(1,10,14,46)$ & 11748 & 24 & $20(12), 17,3$ & 328229 & 7389 & 3687 \\
\hline 46 & $(1,10,16,34)$ & 14994 & 36 & $36(18), 29,5$ & 198765 & 503 & 173 \\
\hline 47 & $(1,10,18,28)$ & 4698 & 12 & $12(3), 6,2$ & 19344 & 32 & 14 \\
\hline 48 & $(1,10,19,26)$ & 2280 & 6 & $4,0,0$ & 0 & 0 & 0 \\
\hline 49 & $(1,10,22,22)$ & 26532 & 72 & $50(20), 39,10$ & 499730 & 15055 & 2780 \\
\hline 50 & $(1,11,11,76)$ & 9828 & 13 & $2,1,0$ & 96 & 0 & 0 \\
\hline 51 & $(1,12,12,40)$ & 12138 & 28 & $10(2), 8,1$ & 22942 & 8 & 2 \\
\hline 52 & $(1,12,13,33)$ & 595 & 1 & $1,0,0$ & 0 & 0 & 0 \\
\hline 53 & $(1,12,19,19)$ & 10500 & 21 & $4,0,0$ & 0 & 0 & 0 \\
\hline 54 & $(1,13,13,28)$ & 19845 & 45 & $10,5,0$ & 1854 & 0 & 0 \\
\hline 55 & $(1,13,18,18)$ & 3220 & 10 & $4,1,0$ & 20 & 0 & 0 \\
\hline 56 & $(1,14,14,22)$ & 5336 & 16 & $14(1), 10,2$ & 17112 & 63 & 23 \\
\hline
\end{tabular}


Appendix: Tables

Table 4.2: Summary results for all Gepner Models

\begin{tabular}{|c|c|c|c|c|c|c|c|}
\hline nr. & Tensor & Prim & S.C. & MIPF & Intersect. & Sol. & SM spectra \\
\hline 57 & $(1,16,16,16)$ & 33210 & 108 & $26(5), 17,5$ & 125476 & 9204 & 494 \\
\hline 58 & $(2,3,19,418)$ & 6320 & 2 & $2,0,0$ & 0 & 0 & 0 \\
\hline 59 & $(2,3,20,218)$ & 6972 & 4 & $6(4), 4,0$ & 315210 & 0 & 0 \\
\hline 60 & $(2,3,22,118)$ & 8232 & 8 & $5(4), 4,1$ & 180431 & 6 & 4 \\
\hline 61 & $(2,3,23,98)$ & 9120 & 10 & $4(1), 1,0$ & 1204 & 0 & 0 \\
\hline 62 & $(2,3,26,68)$ & 3036 & 4 & $6(4), 4,0$ & 18416 & 0 & 0 \\
\hline 63 & $(2,3,28,58)$ & 13340 & 20 & $12(8), 9,4$ & 142742 & 482 & 176 \\
\hline 64 & $(2,3,34,43)$ & 1232 & 2 & $2,0,0$ & 0 & 0 & 0 \\
\hline 65 & $(2,3,38,38)$ & 22920 & 40 & $10(8), 9,2$ & 151792 & 1044 & 216 \\
\hline 66 & $(2,4,11,154)$ & 3540 & 4 & $6(4), 4,0$ & 2304160 & 0 & 0 \\
\hline 67 & $(2,4,12,82)$ & 4160 & 8 & $30(26), 26,12$ & 5133558 & 598 & 294 \\
\hline 68 & $(2,4,13,58)$ & 4830 & 12 & $12(7), 9,1$ & 468648 & 146 & 69 \\
\hline 69 & $(2,4,14,46)$ & 5340 & 16 & $27(24), 25,13$ & 1918601 & 17411 & 5607 \\
\hline 70 & $(2,4,16,34)$ & 7140 & 24 & $60(48), 54,23$ & 3700006 & 218598 & 45055 \\
\hline 71 & $(2,4,18,28)$ & 2320 & 8 & $30(23), 25,15$ & 745644 & 801 & 360 \\
\hline 72 & $(2,4,19,26)$ & 1100 & 4 & $6(2), 3,0$ & 23872 & 0 & 0 \\
\hline 73 & $(2,4,22,22)$ & 12060 & 48 & $54(39), 51,25$ & 3403934 & 423560 & 43532 \\
\hline 74 & $(2,5,8,138)$ & 2862 & 4 & $6(3), 4,0$ & 191424 & 0 & 0 \\
\hline 75 & $(2,5,10,40)$ & 1230 & 4 & $6(1), 3,0$ & 5502 & 0 & 0 \\
\hline 76 & $(2,5,12,26)$ & 6006 & 28 & $12,7,3$ & 34744 & 2426 & 431 \\
\hline 77 & $(2,6,7,70)$ & 3024 & 8 & $5(1), 3,0$ & 28234 & 0 & 0 \\
\hline 78 & $(2,6,8,38)$ & 3780 & 16 & $27(18), 22,7$ & 323662 & 6313 & 1368 \\
\hline 79 & $(2,6,10,22)$ & 5544 & 32 & $59(20), 53,27$ & 361546 & 18964 & 3624 \\
\hline 80 & $(2,6,14,14)$ & 9744 & 64 & $87(20), 71,30$ & 758636 & 62856 & 5424 \\
\hline 81 & $(2,7,7,34)$ & 4032 & 18 & $6,0,0$ & 0 & 0 & 0 \\
\hline 82 & $(2,7,10,16)$ & 2040 & 12 & $12,6,1$ & 10504 & 4 & 1 \\
\hline 83 & $(2,8,8,18)$ & 6480 & 40 & $44(3), 32,16$ & 1019592 & 222006 & 17311 \\
\hline 84 & $(2,8,10,13)$ & 630 & 4 & $6,3,0$ & 1320 & 0 & 0 \\
\hline 85 & $(2,10,10,10)$ & 12000 & 96 & $92(7), 71,34$ & 850844 & 137472 & 9878 \\
\hline 86 & $(3,3,9,108)$ & 2900 & 5 & $2,0,0$ & 0 & 0 & 0 \\
\hline 87 & $(3,3,10,58)$ & 3280 & 10 & $4,1,0$ & 124 & 0 & 0 \\
\hline 88 & $(3,3,12,33)$ & 1700 & 5 & $2,0,0$ & 0 & 0 & 0 \\
\hline 89 & $(3,3,13,28)$ & 6300 & 25 & $6,2,0$ & 118 & 0 & 0 \\
\hline 90 & $(3,3,18,18)$ & 9200 & 50 & $12,4,0$ & 1492 & 0 & 0 \\
\hline 91 & $(3,4,6,118)$ & 2100 & 4 & $6(3), 3,0$ & 176520 & 0 & 0 \\
\hline 92 & $(3,4,7,43)$ & 1584 & 3 & $2,0,0$ & 0 & 0 & 0 \\
\hline 93 & $(3,4,8,28)$ & 3280 & 20 & $12(1), 7,0$ & 3348 & 0 & 0 \\
\hline 94 & $(3,4,10,18)$ & 540 & 4 & $6,0,0$ & 0 & 0 & 0 \\
\hline 95 & $(3,4,13,13)$ & 4410 & 15 & $4,0,0$ & 0 & 0 & 0 \\
\hline 96 & $(3,5,5,68)$ & 2394 & 7 & $2,0,0$ & 0 & 0 & 0 \\
\hline 97 & $(3,6,6,18)$ & 1064 & 8 & $10,1,0$ & 88 & 0 & 0 \\
\hline 98 & $(3,8,8,8)$ & 9200 & 100 & $18,11,2$ & 5406 & 96 & 4 \\
\hline 99 & $(4,4,5,40)$ & 2322 & 12 & $10,6,0$ & 56000 & 0 & 0 \\
\hline 100 & $(4,4,6,22)$ & 3150 & 24 & $44(9), 33,15$ & 465222 & 51448 & 8737 \\
\hline 101 & $(4,4,7,16)$ & 3888 & 36 & $26(1), 17,6$ & 93764 & 2590 & 59 \\
\hline 102 & $(4,4,8,13)$ & 1218 & 12 & $10(2), 7,0$ & 3682 & 0 & 0 \\
\hline 103 & $(4,4,10,10)$ & 7200 & 72 & $110(3), 74,15$ & 999730 & 277752 & 9983 \\
\hline 104 & $(4,5,5,19)$ & 1890 & 7 & $2,0,0$ & 0 & 0 & 0 \\
\hline 105 & $(4,6,6,10)$ & 1540 & 16 & $54,22,3$ & 6874 & 64 & 6 \\
\hline
\end{tabular}


Susy SM spectra from RCFT Orientifolds

Table 4.2: Summary results for all Gepner Models

\begin{tabular}{|c|c|c|c|c|c|c|c|}
\hline nr. & Tensor & Prim & S.C. & MIPF & Intersect. & Sol. & SM spectra \\
\hline 106 & $(4,7,7,7)$ & 5184 & 27 & $6,0,0$ & 0 & 0 & 0 \\
\hline 107 & $(5,5,5,12)$ & 6615 & 49 & $5,0,0$ & 0 & 0 & 0 \\
\hline 108 & $(6,6,6,6)$ & 9632 & 128 & $76,44,10$ & 174232 & 70864 & 1310 \\
\hline 109 & $(1,1,2,11,154)$ & 2124 & 6 & $4,0,0$ & 0 & 0 & 0 \\
\hline 110 & $(1,1,2,12,82)$ & 2496 & 12 & $12,0,0$ & 0 & 0 & 0 \\
\hline 111 & $(1,1,2,13,58)$ & 2898 & 18 & $10,0,0$ & 0 & 0 & 0 \\
\hline 112 & $(1,1,2,14,46)$ & 3204 & 24 & $10,2,0$ & 788 & 0 & 0 \\
\hline 113 & $(1,1,2,16,34)$ & 4284 & 36 & $30,8,0$ & 260 & 0 & 0 \\
\hline 114 & $(1,1,2,18,28)$ & 1392 & 12 & $12,0,0$ & 0 & 0 & 0 \\
\hline 115 & $(1,1,2,19,26)$ & 660 & 6 & $4,0,0$ & 0 & 0 & 0 \\
\hline 116 & $(1,1,2,22,22)$ & 7236 & 72 & $26,7,0$ & 3864 & 0 & 0 \\
\hline 117 & $(1,1,3,6,118)$ & 1260 & 6 & $4,0,0$ & 0 & 0 & 0 \\
\hline 118 & $(1,1,3,7,43)$ & 1584 & 9 & $5,0,0$ & 0 & 0 & 0 \\
\hline 119 & $(1,1,3,8,28)$ & 2010 & 30 & $8,1,0$ & 2 & 0 & 0 \\
\hline 120 & $(1,1,3,10,18)$ & 324 & 6 & $4,0,0$ & 0 & 0 & 0 \\
\hline 121 & $(1,1,3,13,13)$ & 4410 & 45 & $10,1,0$ & 54 & 0 & 0 \\
\hline 122 & $(1,1,4,5,40)$ & 1431 & 18 & $10,0,0$ & 0 & 0 & 0 \\
\hline 123 & $(1,1,4,6,22)$ & 1890 & 36 & $30,0,0$ & 0 & 0 & 0 \\
\hline 124 & $(1,1,4,7,16)$ & 2484 & 54 & $40,1,0$ & 2 & 0 & 0 \\
\hline 125 & $(1,1,4,8,13)$ & 819 & 18 & $10,0,0$ & 0 & 0 & 0 \\
\hline 126 & $(1,1,4,10,10)$ & 4320 & 108 & $96,6,0$ & 652 & 0 & 0 \\
\hline 127 & $(1,1,5,5,19)$ & 1890 & 21 & $4,0,0$ & 0 & 0 & 0 \\
\hline 128 & $(1,1,6,6,10)$ & 924 & 24 & $20,1,0$ & 16 & 0 & 0 \\
\hline 129 & $(1,1,7,7,7)$ & 5184 & 81 & $19,2,0$ & 8 & 0 & 0 \\
\hline 130 & $(1,2,2,5,40)$ & 492 & 4 & $6,0,0$ & 0 & 0 & 0 \\
\hline 131 & $(1,2,2,6,22)$ & 1512 & 32 & $31,7,0$ & 3434 & 0 & 0 \\
\hline 132 & $(1,2,2,7,16)$ & 816 & 12 & $12,0,0$ & 0 & 0 & 0 \\
\hline 133 & $(1,2,2,8,13)$ & 252 & 4 & $6,0,0$ & 0 & 0 & 0 \\
\hline 134 & $(1,2,2,10,10)$ & 3288 & 96 & $120,22,1$ & 7566 & 72 & 2 \\
\hline 135 & $(1,2,3,3,58)$ & 920 & 10 & $4,0,0$ & 0 & 0 & 0 \\
\hline 136 & $(1,2,3,4,18)$ & 160 & 4 & $6,0,0$ & 0 & 0 & 0 \\
\hline 137 & $(1,2,4,4,10)$ & 2250 & 72 & $118,19,4$ & 10920 & 730 & 99 \\
\hline 138 & $(1,2,4,6,6)$ & 420 & 16 & $27,0,0$ & 0 & 0 & 0 \\
\hline 139 & $(1,3,3,3,13)$ & 1400 & 25 & $4,0,0$ & 0 & 0 & 0 \\
\hline 140 & $(1,3,3,4,8)$ & 260 & 10 & $4,0,0$ & 0 & 0 & 0 \\
\hline 141 & $(1,4,4,4,4)$ & 4266 & 216 & $112,11,2$ & 5552 & 172 & 6 \\
\hline 142 & $(2,2,2,3,18)$ & 1040 & 32 & $27,2,0$ & 1168 & 0 & 0 \\
\hline 143 & $(2,2,2,4,10)$ & 1520 & 64 & $230,60,7$ & 48876 & 4832 & 92 \\
\hline 144 & $(2,2,2,6,6)$ & 3024 & 128 & $305,101,6$ & 111080 & 10304 & 95 \\
\hline 145 & $(2,2,3,3,8)$ & 720 & 20 & $12,0,0$ & 0 & 0 & 0 \\
\hline 146 & $(2,2,4,4,4)$ & 1500 & 48 & $180,5,0$ & 4640 & 0 & 0 \\
\hline 147 & $(3,3,3,3,3)$ & 4000 & 125 & $8,0,0$ & 0 & 0 & 0 \\
\hline 148 & $(1,1,1,1,5,40)$ & 972 & 27 & $8,0,0$ & 0 & 0 & 0 \\
\hline 149 & $(1,1,1,1,6,22)$ & 1134 & 54 & $16,0,0$ & 0 & 0 & 0 \\
\hline 150 & $(1,1,1,1,7,16)$ & 1944 & 81 & $34,1,1$ & 6 & 3 & 2 \\
\hline 151 & $(1,1,1,1,8,13)$ & 756 & 27 & $8,0,0$ & 0 & 0 & 0 \\
\hline 152 & $(1,1,1,1,10,10)$ & 2592 & 162 & $58,0,0$ & 0 & 0 & 0 \\
\hline 153 & $(1,1,1,2,3,18)$ & 96 & 6 & $4,0,0$ & 0 & 0 & 0 \\
\hline 154 & $(1,1,1,2,4,10)$ & 1350 & 108 & $72,0,0$ & 0 & 0 & 0 \\
\hline
\end{tabular}


Appendix: Tables

Table 4.2: Summary results for all Gepner Models

\begin{tabular}{|l|c|c|c|c|c|c|c|}
\hline nr. & Tensor & Prim & S.C. & MIPF & Intersect. & Sol. & SM spectra \\
\hline 155 & $(1,1,1,2,6,6)$ & 252 & 24 & $10,0,0$ & 0 & 0 & 0 \\
156 & $(1,1,1,3,3,8)$ & 240 & 15 & $4,0,0$ & 0 & 0 & 0 \\
157 & $(1,1,1,4,4,4)$ & 2673 & 324 & $142,0,0$ & 0 & 0 & 0 \\
158 & $(1,1,2,2,2,10)$ & 912 & 96 & $52,0,0$ & 0 & 0 & 0 \\
159 & $(1,1,2,2,4,4)$ & 900 & 72 & $110,0,0$ & 0 & 0 & 0 \\
160 & $(1,2,2,2,2,4)$ & 440 & 64 & $138,0,0$ & 0 & 0 & 0 \\
161 & $(2,2,2,2,2,2)$ & 2944 & 512 & $1031,10,0$ & 448 & 0 & 0 \\
162 & $(1,1,1,1,1,2,10)$ & 810 & 162 & $34,0,0$ & 0 & 0 & 0 \\
163 & $(1,1,1,1,1,4,4)$ & 1944 & 486 & $156,0,0$ & 0 & 0 & 0 \\
164 & $(1,1,1,1,2,2,4)$ & 540 & 108 & $48,0,0$ & 0 & 0 & 0 \\
165 & $(1,1,1,2,2,2,2)$ & 264 & 96 & $46,0,0$ & 0 & 0 & 0 \\
166 & $(1,1,1,1,1,1,1,4)$ & 2187 & 729 & $124,0,0$ & 0 & 0 & 0 \\
167 & $(1,1,1,1,1,1,2,2)$ & 324 & 162 & $24,0,0$ & 0 & 0 & 0 \\
168 & $(1,1,1,1,1,1,1,1,1)$ & 2187 & 2187 & $152,0,0$ & 0 & 0 & 0 \\
\hline
\end{tabular}

Table 4.3: All MIPFs with solutions

\begin{tabular}{|l|c|c|c|c|}
\hline Tensor & $\left(h_{11}, h_{21}, S\right)$ & Boundaries & Nr. & Types \\
\hline$(1,6,28,118)$ & $(24,84,429)$ & 2400 & 6 & $(0,0,0,0,50,0,0)$ \\
& $(75,75,565)$ & 6090 & 2 & $(0,0,0,2,8,0,0)$ \\
$(1,6,30,94)$ & $(24,84,425)$ & 1980 & 6 & $(0,0,0,0,144,0,0)$ \\
$(1,6,34,70)$ & $(29,125,557)$ & 3312 & 10 & $(0,0,3,2,0,0,0)$ \\
& $(14,98,451)$ & 1656 & 9 & $(0,0,0,0,1173,0,0)$ \\
& $(43,91,509)$ & 4464 & 2 & $(0,0,0,2,11,0,0)$ \\
$(1,6,38,58)$ & $(29,53,351)$ & 1520 & 6 & $(0,0,0,0,18,0,0)$ \\
$(1,6,46,46)$ & $(19,163,649)$ & 2968 & 12 & $(0,0,81,0,11,0,0)$ \\
& $(9,129,525)$ & 1484 & 10 & $(0,0,0,0,16294,0,0)$ \\
& $(27,123,569)$ & 3944 & 2 & $(0,0,4,20,28,0,0)$ \\
& $(19,67,377)$ & 2968 & 13 & $(0,0,6,0,16,0,0)$ \\
& $(59,59,449)$ & 7888 & 4 & $(0,0,0,0,1,0,0)$ \\
$(1,7,34,34)$ & $(23,95,437)$ & 2136 & 10 & $(0,0,0,2,0,0,0)$ \\
& $(29,77,457)$ & 3696 & 2 & $(0,0,2,0,4,0,0)$ \\
$(1,8,14,238)$ & $(53,53,443)$ & 3252 & 4 & $(0,0,0,0,2,0,0)$ \\
$(1,8,18,58)$ & $(38,62,377)$ & 2538 & 2 & $(0,0,0,0,1,0,0)$ \\
$(1,8,28,28)$ & $(17,95,419)$ & 1894 & 2 & $(0,0,110,20,54,0,0)$ \\
& $(29,29,251)$ & 2316 & 12 & $(0,0,4,0,0,0,0)$ \\
$(1,10,14,46)$ & $(8,68,315)$ & 728 & 8 & $(0,0,0,0,3423,0,0)$ \\
& $(17,41,259)$ & 1540 & 11 & $(0,0,123,0,92,0,0)$ \\
$(1,10,16,34)$ & $(35,59,369)$ & 2000 & 2 & $(0,0,0,16,6,0,0)$ \\
& $(13,97,405)$ & 1088 & 20 & $(0,0,8,0,16,0,0)$ \\
$(1,10,22,22)$ & $(29,65,355)$ & 1666 & 22 & $(0,0,20,6,44,10,0)$ \\
& $(20,32,241)$ & 1920 & 25 & $(0,0,6,0,0,0,0)$ \\
& $(20,32,249)$ & 1920 & 29 & $(0,0,9,0,12,0,0)$ \\
& $(38,38,311)$ & 2544 & 7 & $(0,0,0,6,0,0,0)$ \\
& $(10,46,253)$ & 576 & 6 & $(0,0,0,0,12,0,0)$ \\
& $(23,59,343)$ & 1044 & 1 & $(0,0,0,0,2,0,0)$ \\
& $(17,89,387)$ & 1504 & 22 & $(0,0,108,153,130,0,0)$ \\
\hline
\end{tabular}


Table 4.3: All MIPFs with solutions

\begin{tabular}{|c|c|c|c|c|}
\hline Tensor & $\left(h_{11}, h_{21}, S\right)$ & Boundaries & $\mathrm{Nr}$. & Types \\
\hline & $(7,55,263)$ & 1148 & 19 & $(0,0,1227,0,886,0,0)$ \\
\hline & $(19,67,323)$ & 1804 & 34 & $(0,0,0,3,0,0,0)$ \\
\hline & $(19,67,343)$ & 1876 & 35 & $(0,0,0,39,0,0,0)$ \\
\hline & $(22,58,321)$ & 2256 & 30 & $(0,0,0,2,0,0,0)$ \\
\hline & $(13,37,243)$ & 1632 & 29 & $(0,0,8,0,14,0,0)$ \\
\hline & $(25,49,303)$ & 2256 & 44 & $(0,0,0,22,0,0,0)$ \\
\hline & $(27,51,319)$ & 2256 & 31 & $(0,0,31,19,3,0,0)$ \\
\hline & $(41,41,323)$ & 2948 & 36 & $(0,0,0,6,2,0,0)$ \\
\hline$(1,12,12,40)$ & $(25,85,423)$ & 948 & 7 & $(0,0,1,0,1,0,0)$ \\
\hline \multirow[t]{2}{*}{$(1,14,14,22)$} & $(23,23,225)$ & 952 & 9 & $(0,0,13,0,8,0,0)$ \\
\hline & $(31,31,273)$ & 1352 & 13 & $(0,0,0,2,0,0,0)$ \\
\hline \multirow[t]{5}{*}{$(1,16,16,16)$} & $(11,101,401)$ & 1230 & 12 & $(0,0,219,0,35,0,15)$ \\
\hline & $(16,64,325)$ & 2196 & 13 & $(0,0,2,2,0,0,0)$ \\
\hline & $(8,44,227)$ & 1512 & 14 & $(0,0,88,0,88,0,0)$ \\
\hline & $(21,57,301)$ & 2196 & 16 & $(0,0,0,32,0,0,0)$ \\
\hline & $(20,32,243)$ & 1512 & 10 & $(0,0,5,0,0,0,0)$ \\
\hline$(2,3,22,118)$ & $(41,77,463)$ & 2160 & 4 & $(0,0,0,0,3,0,0)$ \\
\hline \multirow[t]{4}{*}{$(2,3,28,58)$} & $(17,101,463)$ & 1408 & 6 & $(0,0,0,0,80,0,0)$ \\
\hline & $(17,101,463)$ & 1472 & 8 & $(0,0,0,0,86,0,0)$ \\
\hline & $(39,87,475)$ & 2552 & 1 & $(0,0,0,0,1,7,0)$ \\
\hline & $(39,87,475)$ & 2668 & 2 & $(0,0,0,0,2,0,0)$ \\
\hline \multirow[t]{2}{*}{$(2,3,38,38)$} & $(11,131,533)$ & 1200 & 7 & $(0,0,0,0,215,0,0)$ \\
\hline & $(23,71,389)$ & 2616 & 8 & $(0,0,0,1,0,0,0)$ \\
\hline \multirow[t]{11}{*}{$(2,4,12,82)$} & $(58,34,371)$ & 2480 & 12 & $(0,0,0,0,4,0,0)$ \\
\hline & $(33,39,325)$ & 1438 & 28 & $(0,0,9,0,71,0,0)$ \\
\hline & $(39,33,325)$ & 1678 & 27 & $(0,0,16,0,13,0,0)$ \\
\hline & $(39,33,325)$ & 1724 & 15 & $(0,0,2,0,4,0,0)$ \\
\hline & $(42,48,357)$ & 1798 & 29 & $(0,0,0,0,19,0,0)$ \\
\hline & $(42,48,357)$ & 1856 & 9 & $(0,0,0,0,12,0,0)$ \\
\hline & $(48,42,357)$ & 2048 & 19 & $(0,0,5,0,1,0,0)$ \\
\hline & $(48,42,357)$ & 2110 & 4 & $(0,0,17,0,53,0,0)$ \\
\hline & $(51,57,447)$ & 2180 & 13 & $(0,0,0,0,12,0,0)$ \\
\hline & $(51,57,447)$ & 2230 & 6 & $(0,0,0,0,2,0,0)$ \\
\hline & $(45,45,350)$ & 2048 & 8 & $(0,0,0,0,2,0,0)$ \\
\hline$(2,4,13,58)$ & $(37,61,413)$ & 1540 & 1 & $(0,0,0,0,5,64,0)$ \\
\hline \multirow[t]{13}{*}{$(2,4,14,46)$} & $(19,67,339)$ & 864 & 24 & $(0,0,0,0,13,0,0)$ \\
\hline & $(20,56,308)$ & 1152 & 25 & $(0,0,134,0,305,0,0)$ \\
\hline & $(21,57,321)$ & 1152 & 23 & $(0,0,0,0,764,0,0)$ \\
\hline & $(26,62,343)$ & 1440 & 18 & $(0,0,14,0,124,2,0)$ \\
\hline & $(29,65,407)$ & 1440 & 1 & $(0,0,5,0,17,20,0)$ \\
\hline & $(56,20,308)$ & 2640 & 21 & $(0,0,0,0,5,0,0)$ \\
\hline & $(62,26,343)$ & 2796 & 7 & $(0,0,1,0,0,0,0)$ \\
\hline & $(20,44,299)$ & 864 & 26 & $(0,0,615,0,835,0,0)$ \\
\hline & $(34,58,367)$ & 1728 & 16 & $(0,0,6,0,57,0,7)$ \\
\hline & $(25,37,287)$ & 1152 & 8 & $(0,0,199,0,716,0,0)$ \\
\hline & $(28,40,309)$ & 1440 & 10 & $(0,0,4,0,221,0,3)$ \\
\hline & $(35,47,329)$ & 1440 & 15 & $(0,0,3,4,10,0,0)$ \\
\hline & $(37,25,287)$ & 1896 & 19 & $(0,0,3,0,1,0,0)$ \\
\hline$(2,4,16,34)$ & $(16,100,440)$ & 1000 & 12 & $(0,0,0,0,742,0,0)$ \\
\hline
\end{tabular}


Appendix: Tables

Table 4.3: All MIPFs with solutions

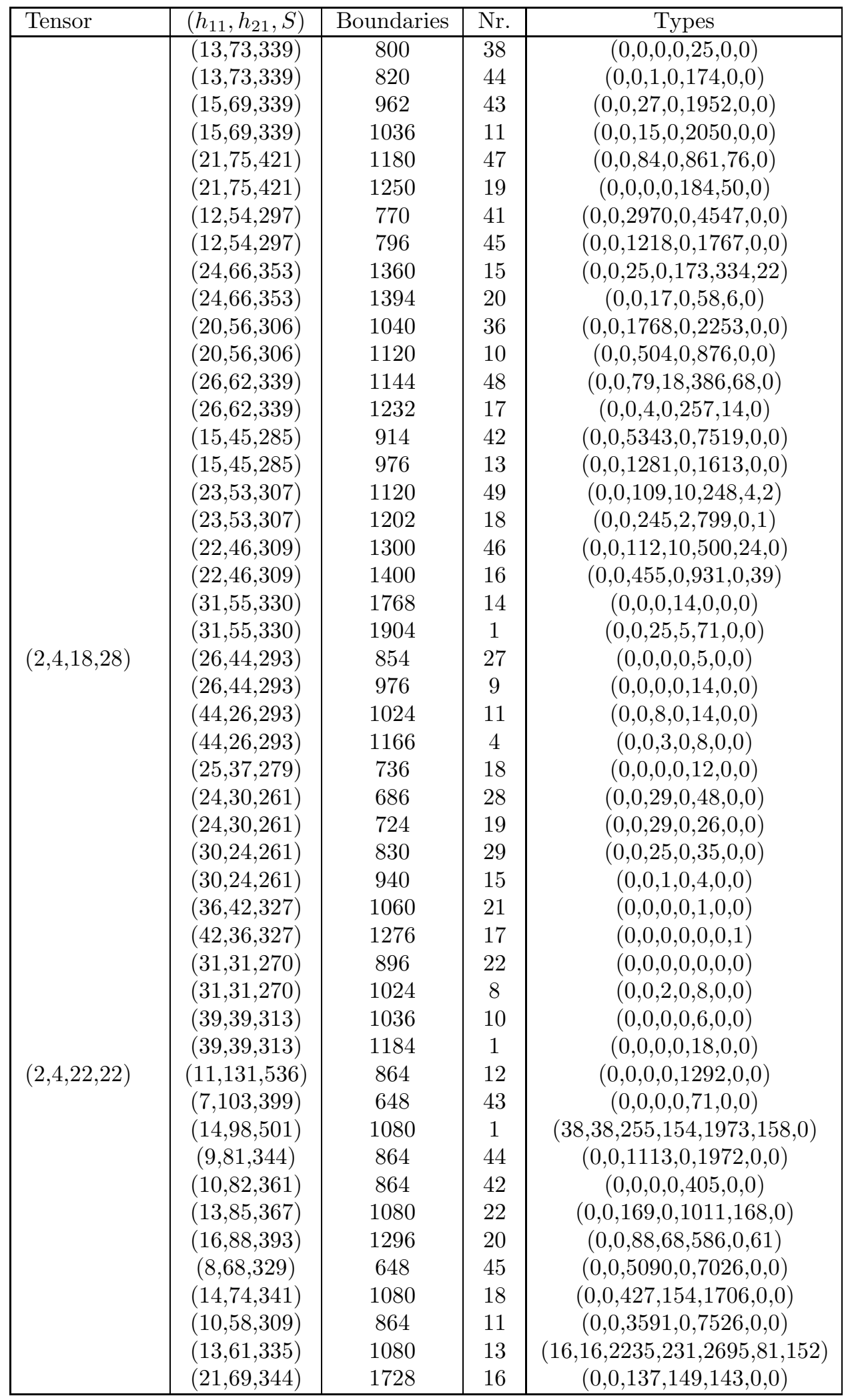


Table 4.3: All MIPFs with solutions

\begin{tabular}{|c|c|c|c|c|}
\hline Tensor & $\left(h_{11}, h_{21}, S\right)$ & Boundaries & $\mathrm{Nr}$. & Types \\
\hline \multirow{49}{*}{$(2,5,12,26)$} & $(20,56,313)$ & 1788 & 48 & $(0,0,138,0,85,0,0)$ \\
\hline & $(16,40,261)$ & 1416 & 46 & $(0,0,86,0,180,0,3)$ \\
\hline & $(19,43,279)$ & 1476 & 49 & $(0,0,274,0,173,0,1)$ \\
\hline & $(28,52,341)$ & 2160 & 2 & $(0,0,0,5,36,104,0)$ \\
\hline & $(52,28,341)$ & 3888 & 35 & $(0,0,0,0,2,0,0)$ \\
\hline & $(20,32,261)$ & 1668 & 17 & $(0,0,383,1,319,1,0)$ \\
\hline & $(32,44,313)$ & 2412 & 30 & $(0,0,0,1,5,0,0)$ \\
\hline & $(23,23,247)$ & 1356 & 21 & $(0,0,0,0,4,0,0)$ \\
\hline & $(31,31,268)$ & 1968 & 47 & $(0,0,43,0,73,0,0)$ \\
\hline & $(31,31,279)$ & 2460 & 19 & $(0,0,0,0,0,4,0)$ \\
\hline & $(32,32,273)$ & 2040 & 28 & $(0,0,27,0,43,0,0)$ \\
\hline & $(39,39,311)$ & 2100 & 31 & $(0,0,0,0,8,0,3)$ \\
\hline & $(8,80,341)$ & 528 & 8 & $(0,0,0,0,32,0,0)$ \\
\hline & $(23,59,327)$ & 780 & 1 & $(0,0,60,0,252,82,0)$ \\
\hline & $(23,59,327)$ & 858 & 2 & $(0,0,0,0,5,0,0)$ \\
\hline & $(14,50,289)$ & 720 & 9 & $(0,0,0,0,506,0,0)$ \\
\hline & $(21,57,333)$ & 1080 & 15 & $(0,0,26,0,14,0,0)$ \\
\hline & $(26,50,313)$ & 1080 & 12 & $(0,0,35,0,30,0,0)$ \\
\hline & $(28,52,331)$ & 1200 & 16 & $(0,0,1,0,74,0,18)$ \\
\hline & $(22,34,265)$ & 720 & 25 & $(0,0,0,0,425,0,0)$ \\
\hline & $(25,37,271)$ & 1080 & 1 & $(0,0,41,0,42,0,0)$ \\
\hline & $(33,33,275)$ & 1440 & 13 & $(0,0,0,0,7,0,0)$ \\
\hline & $(14,62,299)$ & 864 & 56 & $(0,0,150,0,120,0,0)$ \\
\hline & $(15,63,307)$ & 864 & 15 & $(0,0,26,0,33,0,0)$ \\
\hline & $(16,64,331)$ & 864 & 58 & $(0,0,3,0,328,0,0)$ \\
\hline & $(10,46,253)$ & 432 & 13 & $(0,0,0,0,1774,0,0)$ \\
\hline & $(16,40,249)$ & 864 & 22 & $(0,0,75,0,297,0,6)$ \\
\hline & $(19,43,255)$ & 864 & 53 & $(0,0,26,0,36,0,0)$ \\
\hline & $(22,46,275)$ & 864 & 20 & $(0,0,0,0,61,0,0)$ \\
\hline & $(22,46,319)$ & 1152 & 38 & $(0,0,0,0,8,0,0)$ \\
\hline & $(13,25,215)$ & 864 & 55 & $(0,0,0,0,2,0,0)$ \\
\hline & $(15,27,239)$ & 864 & 21 & $(0,0,20,0,18,0,0)$ \\
\hline & $(17,29,223)$ & 1008 & 30 & $(0,0,5,0,0,0,0)$ \\
\hline & $(18,30,223)$ & 1080 & 52 & $(0,0,1,0,7,0,0)$ \\
\hline & $(20,32,241)$ & 1080 & 54 & $(0,0,5,0,53,0,0)$ \\
\hline & $(20,32,261)$ & 1008 & 29 & $(0,0,30,0,60,0,0)$ \\
\hline & $(22,34,253)$ & 1152 & 32 & $(0,0,10,0,50,0,0)$ \\
\hline & $(26,38,283)$ & 1080 & 18 & $(0,0,1,0,2,0,0)$ \\
\hline & $(29,17,223)$ & 1152 & 39 & $(0,0,0,0,11,0,0)$ \\
\hline & $(32,20,241)$ & 1512 & 16 & $(0,0,1,0,5,0,0)$ \\
\hline & $(32,20,261)$ & 1440 & 4 & $(0,0,1,0,1,0,0)$ \\
\hline & $(38,26,275)$ & 1512 & 1 & $(0,0,0,0,3,0,0)$ \\
\hline & $(21,21,235)$ & 864 & 51 & $(0,0,26,0,10,0,0)$ \\
\hline & $(25,25,243)$ & 1008 & 36 & $(0,0,0,0,1,0,0)$ \\
\hline & $(25,25,243)$ & 1152 & 33 & $(0,0,0,0,21,0,0)$ \\
\hline & $(28,28,253)$ & 1008 & 35 & $(0,0,0,0,15,0,0)$ \\
\hline & $(28,28,253)$ & 1440 & 6 & $(0,0,0,0,17,0,0)$ \\
\hline & $(31,31,259)$ & 1440 & 40 & $(0,0,0,0,7,0,0)$ \\
\hline & $(33,33,273)$ & 1728 & 23 & $(0,0,0,0,1,0,0)$ \\
\hline
\end{tabular}


Appendix: Tables

Table 4.3: All MIPFs with solutions

\begin{tabular}{|c|c|c|c|c|}
\hline Tensor & $\left(h_{11}, h_{21}, S\right)$ & Boundaries & $\mathrm{Nr}$. & Types \\
\hline \multirow[t]{30}{*}{$(2,6,14,14)$} & $(8,80,319)$ & 768 & 64 & $(0,0,44,0,73,0,1)$ \\
\hline & $(10,82,355)$ & 768 & 73 & $(0,0,109,0,386,0,0)$ \\
\hline & $(11,83,389)$ & 768 & 14 & $(0,0,7,0,34,0,0)$ \\
\hline & $(9,57,273)$ & 768 & 60 & $(0,0,204,0,808,0,18)$ \\
\hline & $(10,58,271)$ & 768 & 22 & $(0,0,123,0,422,0,4)$ \\
\hline & $(14,62,283)$ & 768 & 19 & $(0,0,4,0,107,0,0)$ \\
\hline & $(14,62,371)$ & 1032 & 17 & $(0,0,77,0,21,0,0)$ \\
\hline & $(13,49,277)$ & 1032 & 71 & $(0,0,56,0,68,0,0)$ \\
\hline & $(13,49,277)$ & 1032 & 80 & $(0,0,56,0,68,0,0)$ \\
\hline & $(8,32,215)$ & 768 & 67 & $(0,0,12,0,52,0,0)$ \\
\hline & $(9,33,233)$ & 768 & 21 & $(0,0,157,0,205,0,0)$ \\
\hline & $(10,34,251)$ & 768 & 62 & $(0,0,107,0,0,0,0)$ \\
\hline & $(11,35,217)$ & 768 & 65 & $(0,0,24,0,23,0,0)$ \\
\hline & $(13,37,233)$ & 768 & 66 & $(0,0,7,0,37,0,0)$ \\
\hline & $(13,37,253)$ & 1032 & 63 & $(0,0,49,0,71,0,0)$ \\
\hline & $(15,39,241)$ & 768 & 69 & $(0,0,39,0,33,0,0)$ \\
\hline & $(15,39,253)$ & 768 & 68 & $(0,0,36,0,8,0,0)$ \\
\hline & $(16,40,263)$ & 1032 & 61 & $(0,0,40,0,77,0,0)$ \\
\hline & $(17,41,265)$ & 1272 & 28 & $(0,0,19,0,13,0,0)$ \\
\hline & $(18,42,263)$ & 1272 & 25 & $(0,0,0,0,7,0,0)$ \\
\hline & $(20,44,255)$ & 1536 & 82 & $(0,0,1,0,2,0,0)$ \\
\hline & $(21,45,273)$ & 1536 & 23 & $(0,0,8,0,14,0,0)$ \\
\hline & $(18,30,221)$ & 1032 & 72 & $(0,0,18,0,389,0,0)$ \\
\hline & $(21,33,243)$ & 1272 & 32 & $(0,0,18,0,9,0,0)$ \\
\hline & $(26,38,271)$ & 1272 & 81 & $(0,0,0,0,49,0,0)$ \\
\hline & $(26,38,271)$ & 1272 & 86 & $(0,0,0,0,49,0,0)$ \\
\hline & $(23,23,225)$ & 1536 & 74 & $(0,0,8,0,8,0,0)$ \\
\hline & $(23,23,229)$ & 1032 & 16 & $(0,0,16,0,20,0,0)$ \\
\hline & $(25,25,265)$ & 1272 & 18 & $(0,0,0,0,34,0,0)$ \\
\hline & $(34,34,347)$ & 1272 & 1 & $(0,0,0,0,12,0,8)$ \\
\hline$(2,7,10,16)$ & $(31,31,271)$ & 544 & 9 & $(0,0,0,0,1,0,0)$ \\
\hline \multirow[t]{16}{*}{$(2,8,8,18)$} & $(7,79,325)$ & 468 & 34 & $(0,0,0,0,88,0,0)$ \\
\hline & $(6,72,325)$ & 528 & 9 & $(0,0,0,0,140,0,0)$ \\
\hline & $(12,66,315)$ & 648 & 37 & $(0,0,53,0,379,52,0)$ \\
\hline & $(12,66,315)$ & 702 & 17 & $(0,0,10,0,83,4,0)$ \\
\hline & $(6,48,249)$ & 414 & 33 & $(0,0,2107,0,2552,0,0)$ \\
\hline & $(6,48,249)$ & 456 & 35 & $(0,0,345,0,344,0,0)$ \\
\hline & $(13,49,249)$ & 504 & 28 & $(0,0,1194,0,3295,0,0)$ \\
\hline & $(13,49,249)$ & 576 & 8 & $(0,0,649,0,1683,0,0)$ \\
\hline & $(13,49,259)$ & 756 & 36 & $(0,0,394,8,781,114,0)$ \\
\hline & $(13,49,259)$ & 864 & 14 & $(0,0,562,1,751,0,6)$ \\
\hline & $(21,57,313)$ & 588 & 10 & $(0,0,55,0,126,0,0)$ \\
\hline & $(21,57,313)$ & 672 & 1 & $(0,0,0,0,187,4,0)$ \\
\hline & $(16,46,257)$ & 576 & 11 & $(0,0,101,0,113,0,0)$ \\
\hline & $(16,46,257)$ & 654 & 15 & $(0,0,127,0,203,0,0)$ \\
\hline & $(28,34,257)$ & 1134 & 16 & $(0,0,0,0,11,4,0)$ \\
\hline & $(28,34,257)$ & 1296 & 2 & $(0,0,22,0,10,0,0)$ \\
\hline \multirow[t]{2}{*}{$(2,10,10,10)$} & $(3,105,381)$ & 320 & 44 & $(0,0,0,0,67,0,0)$ \\
\hline & $(3,105,381)$ & 328 & 57 & $(0,0,0,0,377,0,0)$ \\
\hline
\end{tabular}


Table 4.3: All MIPFs with solutions

\begin{tabular}{|c|c|c|c|c|}
\hline Tensor & $\left(h_{11}, h_{21}, S\right)$ & Boundaries & $\mathrm{Nr}$. & Types \\
\hline & $(7,91,371)$ & 640 & 54 & $(0,0,58,0,62,0,0)$ \\
\hline & $(7,91,371)$ & 656 & 11 & $(0,0,37,0,169,0,0)$ \\
\hline & $(3,69,277)$ & 320 & 46 & $(0,0,225,0,764,0,0)$ \\
\hline & $(3,69,277)$ & 328 & 56 & $(0,0,771,0,1060,0,0)$ \\
\hline & $(7,67,279)$ & 640 & 52 & $(0,0,100,0,148,0,3)$ \\
\hline & $(7,67,279)$ & 656 & 63 & $(0,0,89,11,495,0,0)$ \\
\hline & $(9,63,265)$ & 984 & 26 & $(0,0,1,0,2,0,0)$ \\
\hline & $(11,59,319)$ & 824 & 13 & $(0,0,12,0,0,0,0)$ \\
\hline & $(11,59,319)$ & 832 & 55 & $(0,0,18,0,0,0,0)$ \\
\hline & $(7,43,231)$ & 824 & 64 & $(0,0,21,0,49,0,0)$ \\
\hline & $(7,43,231)$ & 832 & 51 & $(0,0,94,0,23,0,0)$ \\
\hline & $(9,45,243)$ & 824 & 65 & $(0,0,265,0,651,0,1)$ \\
\hline & $(9,45,243)$ & 832 & 53 & $(0,0,98,14,85,0,0)$ \\
\hline & $(13,49,251)$ & 1112 & 66 & $(0,0,22,1,76,0,0)$ \\
\hline & $(13,49,251)$ & 1120 & 18 & $(0,0,65,4,73,2,0)$ \\
\hline & $(15,51,271)$ & 1312 & 16 & $(0,0,1,0,3,0,0)$ \\
\hline & $(9,33,231)$ & 640 & 50 & $(0,0,442,0,424,0,0)$ \\
\hline & $(9,33,231)$ & 656 & 62 & $(0,0,170,0,230,0,0)$ \\
\hline & $(17,41,247)$ & 1112 & 67 & $(0,0,0,0,11,0,0)$ \\
\hline & $(17,41,247)$ & 1120 & 21 & $(0,0,9,0,11,0,0)$ \\
\hline & $(9,27,193)$ & 984 & 25 & $(0,0,1,0,2,0,0)$ \\
\hline & $(13,25,207)$ & 640 & 48 & $(0,0,1,0,0,0,0)$ \\
\hline & $(17,29,215)$ & 824 & 12 & $(0,0,138,0,172,0,0)$ \\
\hline & $(17,29,215)$ & 832 & 49 & $(0,0,138,0,516,0,0)$ \\
\hline & $(19,31,231)$ & 1648 & 19 & $(0,0,0,0,2,0,0)$ \\
\hline & $(19,31,231)$ & 1664 & 59 & $(0,0,0,0,15,0,0)$ \\
\hline & $(19,31,235)$ & 1112 & 15 & $(0,0,72,0,56,0,0)$ \\
\hline & $(19,31,235)$ & 1120 & 24 & $(0,0,10,0,21,0,0)$ \\
\hline & $(27,39,299)$ & 1112 & 14 & $(0,0,0,0,32,0,0)$ \\
\hline & $(27,39,299)$ & 1120 & 1 & $(0,0,6,0,62,0,0)$ \\
\hline & $(35,23,243)$ & 2224 & 22 & $(0,0,0,0,4,0,0)$ \\
\hline & $(35,23,243)$ & 2240 & 2 & $(0,0,0,0,1,0,0)$ \\
\hline \multirow[t]{2}{*}{$(3,8,8,8)$} & $(11,47,283)$ & 880 & 9 & $(0,0,0,0,0,0,0)$ \\
\hline & $(13,25,213)$ & 1120 & 12 & $(0,0,0,0,3,0,0)$ \\
\hline \multirow[t]{15}{*}{$(4,4,6,22)$} & $(9,57,289)$ & 546 & 37 & $(0,0,0,0,7,0,0)$ \\
\hline & $(13,61,289)$ & 330 & 12 & $(0,0,0,0,544,0,0)$ \\
\hline & $(6,42,223)$ & 354 & 30 & $(0,0,1147,0,2200,0,0)$ \\
\hline & $(12,48,255)$ & 510 & 11 & $(0,0,0,0,480,110,0)$ \\
\hline & $(12,48,256)$ & 408 & 28 & $(0,0,0,0,78,0,0)$ \\
\hline & $(9,33,211)$ & 438 & 8 & $(0,0,612,0,621,0,0)$ \\
\hline & $(13,37,224)$ & 600 & 10 & $(0,0,319,2,366,0,0)$ \\
\hline & $(17,41,243)$ & 570 & 1 & $(0,0,30,21,105,4,0)$ \\
\hline & $(19,43,261)$ & 534 & 14 & $(0,0,54,0,160,0,0)$ \\
\hline & $(19,43,276)$ & 840 & 15 & $(0,0,0,2,0,0,0)$ \\
\hline & $(21,45,283)$ & 420 & 35 & $(0,0,2,0,3,0,0)$ \\
\hline & $(16,28,219)$ & 348 & 13 & $(0,0,289,0,212,0,0)$ \\
\hline & $(18,30,221)$ & 402 & 34 & $(0,0,182,0,485,0,0)$ \\
\hline & $(24,36,261)$ & 954 & 43 & $(0,0,0,0,0,0,3)$ \\
\hline & $(31,31,283)$ & 588 & 17 & $(0,0,10,0,8,0,0)$ \\
\hline
\end{tabular}


Table 4.3: All MIPFs with solutions

\begin{tabular}{|c|c|c|c|c|}
\hline Tensor & $\left(h_{11}, h_{21}, S\right)$ & Boundaries & Nr. & Types \\
\hline \multirow[t]{6}{*}{$(4,4,7,16)$} & $(11,59,283)$ & 288 & 14 & $(0,0,0,0,4,0,0)$ \\
\hline & $(14,50,261)$ & 432 & 15 & $(0,0,5,0,1,0,0)$ \\
\hline & $(15,39,233)$ & 720 & 13 & $(0,0,0,3,0,0,0)$ \\
\hline & $(17,41,283)$ & 864 & 5 & $(0,0,0,0,24,0,0)$ \\
\hline & $(20,32,285)$ & 1008 & 18 & $(0,0,0,0,21,0,0)$ \\
\hline & $(23,23,217)$ & 1008 & 12 & $(0,0,0,3,0,0,0)$ \\
\hline \multirow[t]{15}{*}{$(4,4,10,10)$} & $(4,70,279)$ & 380 & 76 & $(0,0,0,0,16,0,0)$ \\
\hline & $(9,69,319)$ & 308 & 67 & $(0,0,0,0,1,0,0)$ \\
\hline & $(8,62,263)$ & 430 & 22 & $(0,0,0,14,67,0,0)$ \\
\hline & $(10,64,299)$ & 406 & 16 & $(0,0,56,0,34,0,0)$ \\
\hline & $(5,53,232)$ & 440 & 77 & $(0,0,1070,0,1264,0,0)$ \\
\hline & $(9,57,249)$ & 512 & 74 & $(0,0,218,0,273,0,0)$ \\
\hline & $(9,57,252)$ & 640 & 21 & $(0,0,94,32,73,4,0)$ \\
\hline & $(4,46,219)$ & 332 & 14 & $(0,0,1324,0,1409,0,0)$ \\
\hline & $(6,48,223)$ & 296 & 66 & $(0,0,770,0,285,0,0)$ \\
\hline & $(7,43,215)$ & 260 & 79 & $(0,0,1237,0,587,0,0)$ \\
\hline & $(13,49,247)$ & 448 & 80 & $(0,0,15,0,5,0,0)$ \\
\hline & $(10,40,219)$ & 550 & 81 & $(0,0,0,0,59,0,0)$ \\
\hline & $(10,40,223)$ & 744 & 86 & $(0,0,0,0,3,0,0)$ \\
\hline & $(15,39,321)$ & 1056 & 68 & $(0,0,0,0,35,0,0)$ \\
\hline & $(14,20,195)$ & 888 & 101 & $(0,0,0,0,1,0,0)$ \\
\hline \multirow[t]{3}{*}{$(4,6,6,10)$} & $(14,38,229)$ & 416 & 41 & $(0,0,0,0,1,0,1)$ \\
\hline & $(12,24,197)$ & 320 & 50 & $(0,0,0,0,1,0,0)$ \\
\hline & $(24,24,225)$ & 448 & 21 & $(0,0,0,0,3,0,0)$ \\
\hline \multirow[t]{10}{*}{$(6,6,6,6)$} & $(5,69,267)$ & 400 & 7 & $(0,0,4,0,14,0,0)$ \\
\hline & $(3,59,223)$ & 368 & 37 & $(0,0,142,0,226,0,0)$ \\
\hline & $(3,43,207)$ & 400 & 58 & $(0,0,30,0,30,0,0)$ \\
\hline & $(7,47,215)$ & 736 & 55 & $(0,0,0,0,6,0,0)$ \\
\hline & $(5,37,203)$ & 368 & 38 & $(0,0,63,0,100,0,0)$ \\
\hline & $(9,41,211)$ & 800 & 18 & $(0,0,2,0,22,0,2)$ \\
\hline & $(11,27,199)$ & 736 & 56 & $(0,0,0,0,6,0,0)$ \\
\hline & $(13,29,203)$ & 736 & 47 & $(0,0,0,0,4,0,0)$ \\
\hline & $(15,23,199)$ & 1136 & 68 & $(0,0,2,0,2,0,0)$ \\
\hline & $(17,25,203)$ & 1136 & 69 & $(0,0,0,0,2,0,0)$ \\
\hline$(1,2,2,10,10)$ & $(5,29,235)$ & 224 & 19 & $(0,0,2,0,0,0,0)$ \\
\hline \multirow[t]{4}{*}{$(1,2,4,4,10)$} & $(5,53,248)$ & 112 & 76 & $(0,0,1,0,1,0,0)$ \\
\hline & $(5,41,212)$ & 106 & 75 & $(0,0,1,0,1,0,0)$ \\
\hline & $(6,30,196)$ & 106 & 65 & $(0,0,25,0,40,0,0)$ \\
\hline & $(11,35,213)$ & 160 & 67 & $(0,0,0,30,0,0,0)$ \\
\hline \multirow[t]{2}{*}{$(1,4,4,4,4)$} & $(4,40,213)$ & 246 & 58 & $(0,0,0,5,0,0,0)$ \\
\hline & $(13,13,200)$ & 240 & 89 & $(0,0,0,0,1,0,0)$ \\
\hline \multirow[t]{7}{*}{$(2,2,2,4,10)$} & $(9,45,251)$ & 208 & 202 & $(0,0,0,0,2,0,0)$ \\
\hline & $(9,45,251)$ & 320 & 87 & $(0,0,19,0,37,0,0)$ \\
\hline & $(13,25,219)$ & 320 & 101 & $(0,0,14,0,14,0,0)$ \\
\hline & $(15,27,245)$ & 416 & 213 & $(1,1,0,0,0,0,0)$ \\
\hline & $(21,9,211)$ & 272 & 79 & $(0,0,0,0,2,0,0)$ \\
\hline & $(21,9,211)$ & 272 & 109 & $(0,0,0,0,2,0,0)$ \\
\hline & $(21,9,211)$ & 416 & 224 & $(0,0,0,0,2,0,0)$ \\
\hline$(2,2,2,6,6)$ & $(3,51,235)$ & 288 & 106 & $(0,0,31,0,24,0,0)$ \\
\hline
\end{tabular}


Table 4.3: All MIPFs with solutions

\begin{tabular}{|l|c|c|c|c|}
\hline Tensor & $\left(h_{11}, h_{21}, S\right)$ & Boundaries & Nr. & Types \\
\hline & $(9,33,223)$ & 384 & 149 & $(0,0,0,0,1,0,0)$ \\
& $(9,33,223)$ & 384 & 189 & $(0,0,0,0,1,0,0)$ \\
& $(17,17,215)$ & 576 & 135 & $(0,0,0,0,2,0,0)$ \\
& $(17,17,215)$ & 576 & 157 & $(0,0,0,0,2,0,0)$ \\
& $(17,17,215)$ & 576 & 285 & $(0,0,0,0,2,0,0)$ \\
$(1,1,1,1,7,16)$ & $(23,23,217)$ & 72 & 32 & $(0,0,0,2,0,0,0)$ \\
\hline
\end{tabular}


Figure 4.7: Solutions for varying number of chiral families

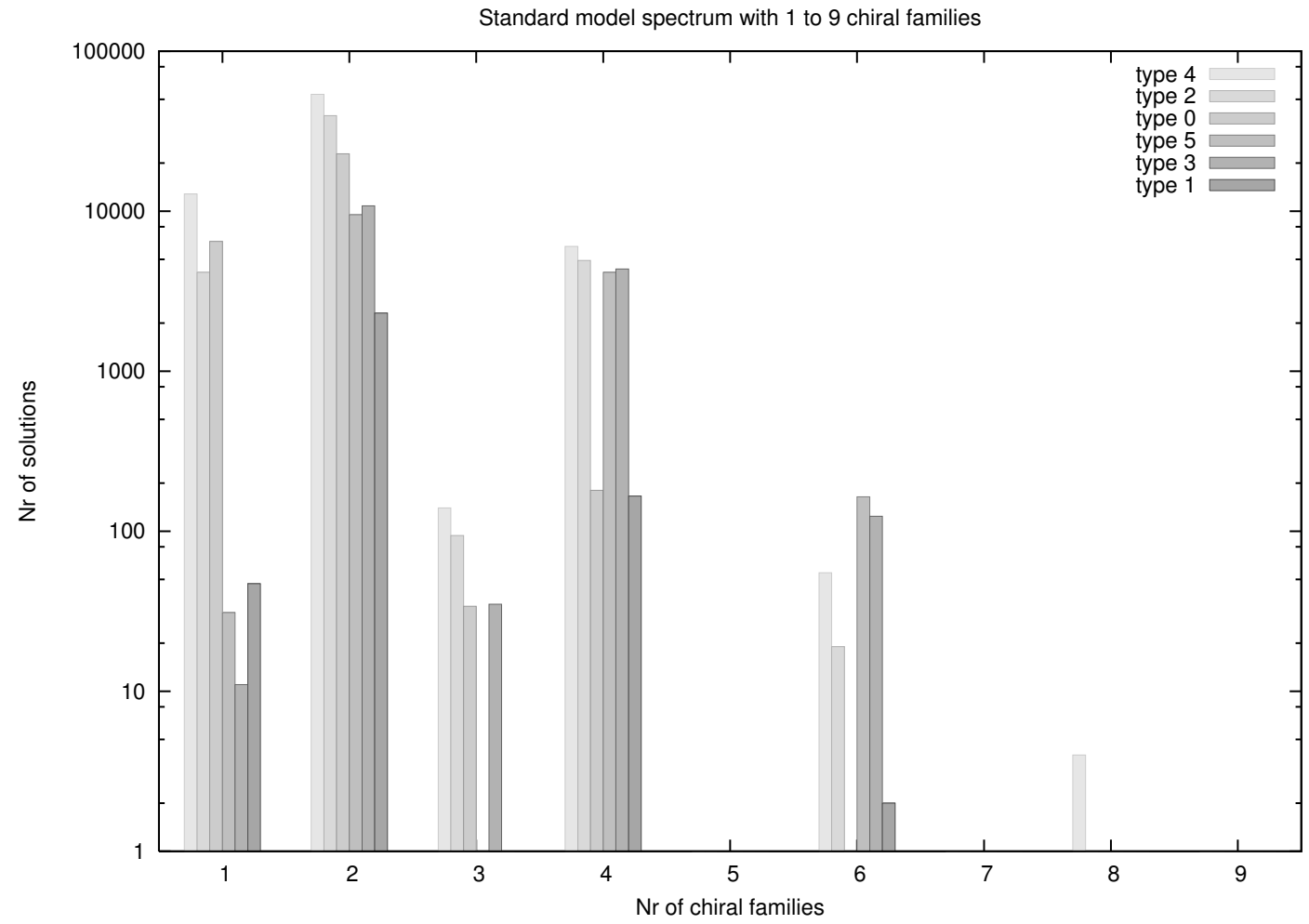

Standard model spectrum with 3 to 9 chiral families

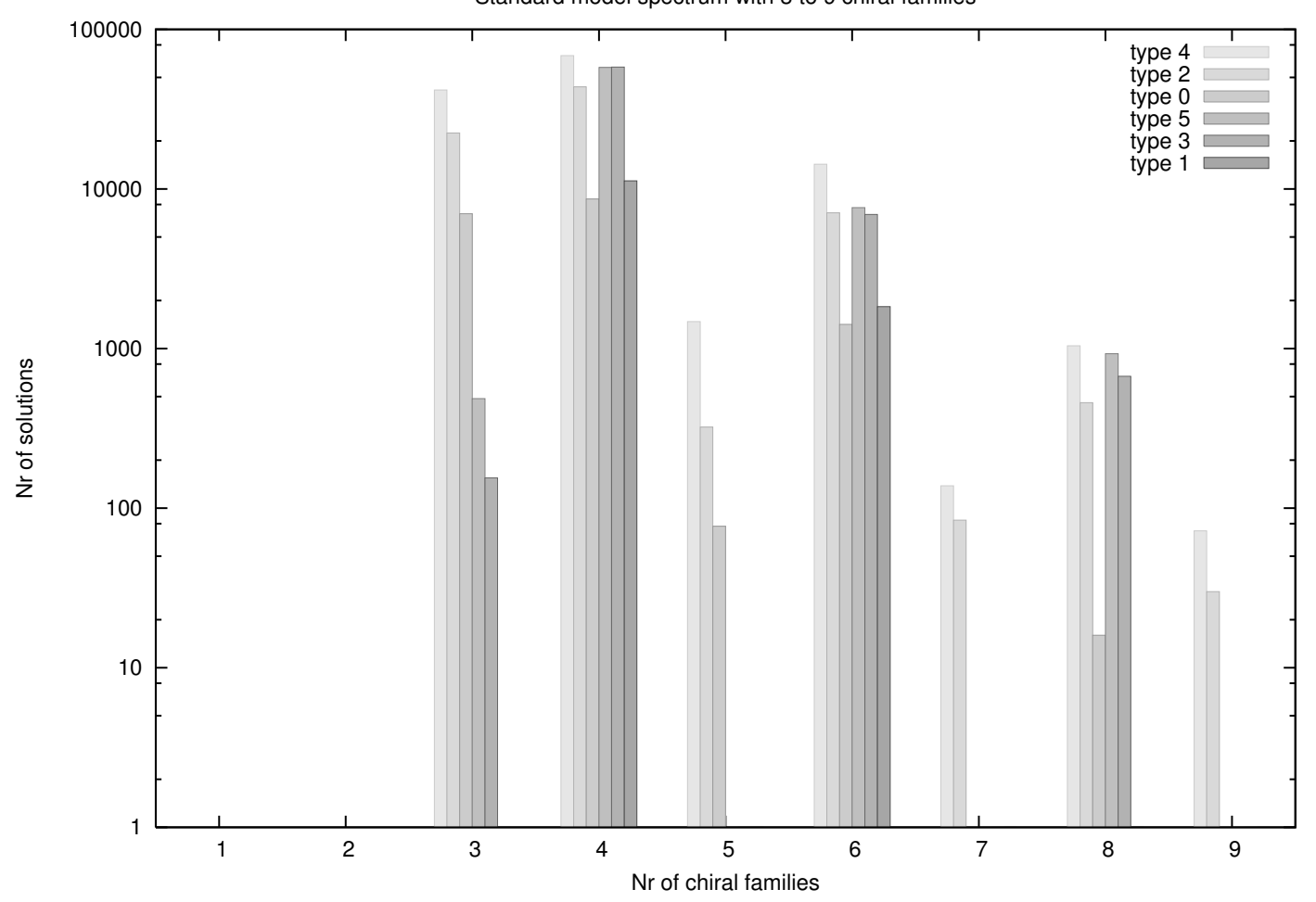


Table 4.1: List of standard model representations that can appear, and their labelling.

\begin{tabular}{|c|c|c|c|c|c|c|c|}
\hline nr. & $U(3)_{\mathrm{a}}$ & $U(2)_{\mathrm{b}}$ & $S p(2)_{\mathrm{b}}$ & $U(1)_{\mathrm{c}}$ & $\begin{array}{l}S O(2)_{\mathrm{c}} \\
S p(2)_{\mathrm{c}}\end{array}$ & $U(1)_{\mathrm{d}}$ & massless particle \\
\hline 1 & $V$ & $V$ & & 0 & & 0 & $(u, d)$ \\
\hline 2 & $V$ & $V^{*}$ & $v$ & 0 & & 0 & $(u, d)$ \\
\hline 3 & $V^{*}$ & 0 & & $V$ & & 0 & $u^{c}$ \\
\hline 4 & $V^{*}$ & 0 & & $V^{*}$ & $V$ & 0 & $d^{c}$ \\
\hline 5 & 0 & $V$ & & 0 & & $V$ & $\left(\nu, e^{-}\right)$ \\
\hline 6 & 0 & $V^{*}$ & $V$ & 0 & & $V$ & $\left(\nu, e^{-}\right)$ \\
\hline 7 & 0 & 0 & & $V$ & & $V^{*}$ & $\nu^{c}$ \\
\hline 8 & 0 & 0 & & $V^{*}$ & $V$ & $V^{*}$ & $e^{+}$ \\
\hline 9 & 0 & $\bar{V}$ & & $V$ & & 0 & $H_{1}$ \\
\hline 10 & 0 & $V$ & & $V^{*}$ & & 0 & $H_{2}$ \\
\hline 11 & $V$ & 0 & & 0 & & $V$ & $\left(3,1,-\frac{1}{3}\right)_{1,1}$ \\
\hline 12 & $V$ & 0 & & 0 & & $V^{*}$ & $\left(3,1, \frac{2}{3}\right)_{1,-1}$ \\
\hline 13 & Adj & 0 & & 0 & & 0 & $(8,1,0)_{0,0}+(1,1,0)_{0,0}$ \\
\hline 14 & $\mathrm{~A}$ & 0 & & 0 & & 0 & $\left(3^{*}, 1, \frac{1}{3}\right)_{2,0}$ \\
\hline 15 & S & 0 & & 0 & & 0 & $\left(6,1, \frac{1}{3}\right)_{2,0}$ \\
\hline 16 & 0 & Adj & - & 0 & & 0 & $(1,3,0)_{0,0}+(1,1,0)_{0,0}$ \\
\hline 17 & 0 & $\mathrm{~A}$ & & 0 & & 0 & $(1,1,0)_{0,0}$ \\
\hline 18 & 0 & S & & 0 & & 0 & $(1,3,0)_{0,0}$ \\
\hline 19 & 0 & 0 & & Adj & - & 0 & $(1,1,0)_{0,0}$ \\
\hline 20 & 0 & 0 & & - & A & 0 & $(1,1,-1)_{0,0}$ \\
\hline 21 & 0 & 0 & & $\mathrm{~S}$ & & 0 & $(1,1,-1)_{0,0}$ \\
\hline 22 & 0 & 0 & & 0 & & Adj & $(1,1,0)_{0,0}$ \\
\hline 23 & 0 & 0 & & 0 & & $\mathrm{~A}$ & - \\
\hline 24 & 0 & 0 & & 0 & & $\mathrm{~S}$ & $(1,1,-1)_{0,2}$ \\
\hline 25 & $\bar{V}$ & 0 & & 0 & & 0 & $\left(3,1, \frac{1}{6}\right)_{1,0}$ \\
\hline 26 & 0 & $V$ & & 0 & & 0 & $(1,2,0)_{0,0}$ \\
\hline 27 & 0 & 0 & & $V$ & & 0 & $\left(1,1,-\frac{1}{2}\right)_{0,0}$ \\
\hline 28 & 0 & 0 & & 0 & & V & $\left(1,1,-\frac{\overline{1}}{2}\right)_{0,1}$ \\
\hline 29 & 0 & 0 & & 0 & & 0 & $(1,1,0)_{0,0}$ \\
\hline
\end{tabular}


Table 4.4: Distribution of chiral standard model types, distinguished by CP group and the $U(2)_{\mathrm{b}}$ anomaly. In the "Higgs" column, " $-2 n$ " implies that there a $n$ supersymmetric Higgs pairs $\left(2, \frac{1}{2}\right)+\left(2,-\frac{1}{2}\right)$ that are chiral with respect to $U(2)_{\mathrm{b}}$ (the sign is just a convention).

\begin{tabular}{|l|c|c|c|c|}
\hline Type & Quarks & Leptons & Higgs & Total \\
\hline 0 & 0 & 0 & 0 & 10564 \\
1 & -3 & 3 & 0 & 32 \\
1 & -9 & 3 & 6 & 1 \\
1 & -9 & 9 & 0 & 22 \\
2 & 0 & 0 & 0 & 49661 \\
3 & -3 & -1 & 4 & 141 \\
3 & -3 & -3 & 6 & 24 \\
3 & -3 & 1 & 2 & 240 \\
3 & -3 & 3 & 0 & 740 \\
3 & -9 & -3 & 12 & 24 \\
3 & -9 & 3 & 6 & 95 \\
3 & -9 & 5 & 4 & 1 \\
3 & -9 & 9 & 0 & 116 \\
4 & 0 & 0 & 0 & 116304 \\
5 & -3 & 1 & 2 & 2 \\
5 & -3 & 3 & 0 & 1507 \\
5 & -9 & 9 & 0 & 46 \\
\hline
\end{tabular}




\section{Chapter 5}

\section{Generalizations}

\subsection{Introduction}

During the past twenty years it has become clear that the information String Theory gives us about the Standard Model (SM) is extremely complex. It does not seem that String Theory, or some selection principle on top of it, will gives us a unique four-dimensional gauge theory that is identical to the Standard Model. On the other hand, there may be non-trivial restrictions on the kind of gauge theories that can emerge from String Theory, and it is not a priori obvious that the Standard Model satisfies those restrictions.

In this situation, there are several approaches one can follow. It would be a tremendous success if one could find a string vacuum that precisely matches all current experimental constraints. For many years it seemed plausible that those constraints would be restrictive enough to reduce the number of vacua to (at most) a single one. The hope was that, having found that vacuum, we would see all the remaining pieces of the puzzle fall into place, and we could start making falsifiable predictions for future experiments.

However, it now seems wishful thinking to believe that this will actually be true. Although reliable estimates cannot be made, naive guesses suggest that the number of vacua meeting all current experimental constraints may well be much larger than 1 . Even in that situation, finding just one of those would be a huge success, at least as an existence proof. But making predictions based on such a vacuum is a rather delicate affair if one does not know the complete ensemble of vacua satisfying all current constraints. This does not mean that no further predictions can be made. There is no reason to expect the moment of discovery of the landscape of string theory, [136], [130] to coincide with the end of such successful theoretical predictions. But all successes of the past (such as the relation between $\alpha$ and $g-2$ ) can be understood in terms of the quantum field theory description of the Standard Model. There may be further successes of this kind, but what one would really hope to find is a genuine string prediction.

At present too little is known about the set of String Theory vacua to be able to say how far this programme can be pushed. One extreme might be that the problem is too (NP)hard for us to solve [54], and that we will have to be satisfied with having a 
certain degree of confidence that the Standard Model does indeed exist somewhere in the landscape, just as we are confident that the DNA molecule is a solution of QED, without being able to write it down explicitly. To accept such an outcome would require, at the very least, some kind of confirmation that String Theory is the correct theory of Quantum Gravity. The other extreme is that the potentially huge set of unfixed degrees of freedom does not actually exist for the Standard Model, or are confined to an irrelevant sub-sector, such as a barely observable ("hidden") sector.

In either case it is clearly essential to expand our knowledge of the landscape of string vacua by all means at our disposal and to understand the possible realizations of vacua that have the SM as a low energy limit. There are two approaches to that end. The first is the top-down approach that constructs string vacua using CFT techniques and then checks whether their low energy limit compares favorably to the SM. This approach has been used extensively in heterotic model building, and more recently in orientifold model building.

The other approach is the bottom up approach that has been especially suited to the orientifold context, $[12,11,4]$. This is because the back-reaction of a brane-configuration comes in at the next order in the coupling constant expansion. It has slowly become clear that in searching for the SM-like vacua, a combination of the two approaches may be the most efficient one.

In this paper, we want to make some modest steps towards understanding the complexity of the landscape, and in particular the different possibilities for realizing SM-like vacua. In particular, it is known that there are several possible embeddings of the SM hypercharge into the orientifold gauge group $[12,11,98,9]$. Such embeddings affect crucial phenomenological properties of the vacua. It is therefore important to analyze such embeddings. For this, instead of focusing on a particular model we will try to broaden the scope as much as possible. In [59], [60] two of the authors presented a detailed investigation of a piece of the landscape that until then was barely accessible: orientifolds of Gepner models. The approach of these papers can be described as a mixture of a top-down and a bottom-up method. On the one hand, exact string solutions were looked for and found. But on the other hand, the kind of solutions that were searched were limited a priori by a choice of a "bottom up" realization of the Standard Model, constructed out of intersection sets of branes.

The scope of the RCFT method, even when restricted to Gepner models, seems to be considerably larger than that of the much more extensively studied orbifold models. Indeed, the first example of a supersymmetric spectrum that matches the standard model exactly (in the chiral sense) was found using an RCFT construction in [59]. This was an amazing eight years after the first steps towards realistic model building with orientifolds were taken [6], using orbifold methods. Since that pioneering paper, the orbifold/orientifold method has been explored extensively by many authors (see [29] and references therein) who succeeded in getting ever closer to the supersymmetric standard model spectrum, until that goal was finally reached in [90] for the $Z_{6}$ orbifold.

\footnotetext{
${ }^{1}$ This terminology is used here only to guide the intuition. In reality the models are described algebraically in terms of annulus coefficients in boundary CFT.
} 
During the same period there has been relatively little work on Gepner orientifolds [7]-[2], and with relatively little success, the first paper finding a chiral spectrum being [38] in 2004. However, it is now clear that the lack of success was due to the fact that until recently only a limited number of partition functions and boundary states was accessible. A recent investigation [85] of $Z_{2} \times Z_{2}$ orbifolds has shown that the three family standard model spectrum is just beyond the limit of statistics in that case. By contrast, with Gepner models more than 200.000 standard model realizations were found in [60], despite the fact that the average success rate is actually lower (empirically, "one in a billion" for $Z_{2} \times Z_{2}$ orbifolds, and about $4 \times 10^{-14}$ for Gepner models).

On the other hand, RCFT methods also have clear disadvantages in comparison to orbifold methods. In particular, they do not allow continuous variations of moduli, and are not suitable for discussions of flux compactifications and moduli stabilization, at least not without radically new ideas. But their larger scope makes them ideally suited for scanning a substantially larger part of the landscape than was possible up to now, provided one focuses only on issues related to spectroscopy. This is precisely our goal in this paper. Our main phenomenological input will be the chiral spectrum of the standard model. Our intention is to loosen considerably the bottom up assumptions made in [60], and investigate a large number of other ways of realizing the standard model with D-branes (or boundary states).

The kind of bottom up models considered in [60] were variations on the model first proposed in [98]. They are characterized by four stacks of branes with a Chan-Paton group $U(3)_{\mathbf{a}} \times U(2)_{\mathbf{b}} \times U(1)_{\mathbf{c}} \times U(1)_{\mathbf{d}}$, with the standard model generator $Y$ embedded as $Y=\frac{1}{6} Q_{\mathbf{a}}-\frac{1}{2} Q_{\mathbf{b}}-\frac{1}{2} Q_{\mathbf{c}}$. The variations include the possibility of choosing the second and third Chan-Paton factor real, and allowing the $B-L$ abelian vector boson to be either massive or massless in the exact string theory. These models have a perturbatively unbroken baryon and lepton number.

Many other brane realizations exist, and some of those have been discussed in the literature. To obtain the results of [60] a huge effort was required in terms of computer time. In principle, this project could be redone for anyone's favorite bottom-up model. However, it seems preferable to try to remove the bias implied by a particular choice of model, and try to repeat the computation assuming as little as possible about the bottomup realization.

In principle, the only feature we assume is the most robust part of what we presently know about the Standard Model: that there are three chiral families of quarks and leptons in the familiar representations of $S U(3) \times S U(2) \times U(1)$. In practice, we still have to make a few concessions. In particular, we will have to limit the number of participating branes and forbid non-chiral mirror pairs of arbitrary charge. This will be discussed in more detail in the next section.

The new features that we do allow include

- Anti-quarks realized as anti-symmetric tensors of $U(3)$

- Charged leptons and neutrinos realized as anti-symmetric tensors 
- Non-standard embeddings of the $Y$-charge

- Embeddings of $Y$ in non-abelian groups

- Strong-Weak unification (e.g. $S U(5))$

- Baryon-lepton unification (e.g. Pati-Salam models)

- Trinification

- Baryon and/or lepton number violation

- Family symmetries

We are not claiming that all of these features are desirable, but our strategy is to allow as many possibilities in an early stage, and leave the final selection to the last stage, so that it will not be necessary to restart the entire search procedure if new insights emerge.

Some of these options may address unsolved problems that occur for the standard realization [98] of the standard model. For example, the perturbatively unbroken lepton number of these models makes it hard to implement a see-saw like mechanism to give small masses to neutrinos. Coupling constant unification, if it is indeed a fundamental feature of nature and not a coincidence, is not automatic in the standard realization, but it would be in $S U(5)$ models. This does not mean that the standard realization cannot accommodate the current experimental values of the couplings constants, but only that the fact that they presently appear to converge (with gaugino contributions taken into account) would be a mere coincidence. We have indeed found some really simple and elegant realizations of $S U(5)$ models.

One of our goals is to analyze which model can be built from a bottom-up point of view, and how many of them can be realized as top-down models. By "bottom-up" we mean here a brane realization that produces the correct chiral standard model spectrum if the gauge group is reduced to $S U(3) \times S U(2) \times U(1)$ (without assuming a particular mechanism for that reduction). On the "top-down" side two types of concepts should be distinguished: standard model brane configurations and solutions to the tadpole conditions. The focus in this paper is on the former, i.e. choices of boundary labels ${ }^{2} \mathbf{a}, \mathbf{b}, \mathbf{c}$ and $\mathbf{d}$ such that with an appropriate choice of the Chan-Paton gauge group and the appropriate embedding of $S U(3) \times S U(2) \times U(1)$ one obtains the standard model. Here we also require that the standard model $U(1)$ generator does not acquire mass due to bilinear axion couplings.

Given such a standard model configuration, there may still be uncancelled tadpoles in RR closed string one-point functions on the disk and the crosscap. Within this context, the only way to cancel them is to add additional hidden matter, except in a few cases where they already cancel among the standard model branes. To see if this can happen is an

\footnotetext{
${ }^{2}$ We label the complete set of boundaries of a given modular invariant partition function of a CFT as $a, b, c, d, \ldots$. The specific boundaries that participate in a Standard Model configuration are denoted as $\mathbf{a}, \mathbf{b}, \mathbf{c}$ and $\mathbf{d}$. We allow a maximum of four (plus a hidden sector), with the first two corresponding to $S U(3)_{\text {color }}$ and $S U(2)_{\text {weak }}$.
} 
extremely time-consuming, and ultimately unsolvable problem. Furthermore for any given brane configuration there may be many ways of cancelling the tadpoles. In the continuum theory, background fluxes, not considered here, contribute to the tadpoles. But perhaps more importantly, the set of boundary states we consider here is limited by the choice of rational CFT. We consider the complete set of boundaries allowed by the RCFT, i.e. all boundaries that respect its chiral algebra. But that chiral algebra is larger than the $N=2$ world-sheet algebra required to describe a geometric Calabi-Yau compactification. Since we get the $c=9$ chiral algebra as a tensor product of minimal $N=2$ algebras, the chiral algebra also contains all differences of the $N=2$ algebras of the factors. If we would reduce the chiral algebra, additional boundary states are allowed, and could contribute to tadpole cancellation. Of course this also allows additional ways of constructing standard model configurations, but we cannot make statements regarding a complete classification there anyway.

It is essentially impossible to conclude, with RCFT techniques alone, that the tadpoles of a certain standard model configuration can not be cancelled. Positive results, on the other hand, imply that one has a valid supersymmetric string vacuum. We see tadpole cancellation therefore mainly as an existence proof of a given string vacuum. Once that proof has been given, we do not continue searching for additional tadpole solutions for the same chiral configuration. This gives an enormous cut-off in computer time. One should keep in mind that for the most frequent chiral model considered in [60], we found a total of 16 million tadpole solutions (about 110000 of them distinct). We now keep only one of those solutions. This also implies that we cannot provide meaningful statistical results regarding tadpole solutions, but only regarding brane configurations.

We summarize briefly our results:

- We develop a detailed classification of allowed embeddings of the SM hypercharge inside the orientifold gauge group. To do this, we classify brane stacks according to how they contribute to the hypercharge. The hypercharge embedding is then characterized by a real variable $x$ which is quantized in half-integral units in genuine non-orientable vacua.

- We produce 19345 chirally distinct top-down SM spectra (before tadpole cancellation) and 1900 chirally distinct models solving the tadpole conditions and realizing the different embeddings.

- We find that the $x=\frac{1}{2}$ hypercharge embedding dominates by far all other choices. The Madrid embedding [98] belongs to this class.

- We produce the first examples of supersymmetric SU(5) and flipped SU(5) orientifold vacua, with the correct chiral spectrum (no extra gauge groups and no exotic $G_{C P}$ chiral states).

- We find some minimal supersymmetric Pati-Salam and trinification vacua. 
- We have examples of spectra (but no tadpole solutions yet) with extended ( $\mathrm{N}=4$ or $\mathrm{N}=8$ ) supersymmetry in the bulk and $\mathrm{N}=1$ supersymmetry on the branes.

- We find corelations between the intersection numbers of a brane with

\subsection{What we are looking for}

Our goal is to search for the most general embedding of the standard model in the ChanPaton gauge group of Gepner Orientifolds.

We first introduce some notation. We denote the full Chan-Paton group as $G_{\mathrm{CP}}$. This is the group obtained directly from the multiplicities of the branes, without taking into account masses generated by two-point axion-gauge boson couplings. We require that the standard model gauge group, $G_{\mathrm{SM}}=S U(3) \times S U(2) \times U(1)_{Y}$ is a subgroup of $G_{\mathrm{CP}}$. Furthermore we require that the generator of $U(1)_{Y}$ does not get a mass from axion-gauge boson couplings.

The main condition we impose on the spectrum is the presence of three families of quarks and leptons, and the absence of chiral exotics. Since chirality can be defined with respect to various groups, and the term "exotics" is used in different senses in the literature, we will define this more precisely. Group-theoretically, the standard-model-like spectra we allow are described as follows. Denote the full set of massless representations of $G_{\mathrm{CP}}$ as $R_{\mathrm{CP}}$. The subset of these representations that is chiral with respect to $G_{\mathrm{CP}}$ is denoted $R_{C P}^{\text {chir }}$. The reduction of these representations to the group $G_{\mathrm{SM}}$ are denoted as $R_{\mathrm{SM}}$ and $R_{\mathrm{SM}}^{\text {chir }}$ respectively. By "reduction" we mean here only that we decompose representations in terms of representations of a subgroup. No assumptions are made at this point regarding dynamical mechanisms (like the Brout-Englert-Higgs mechanism) to achieve such a reduction. Consider now the subset of either $R_{\mathrm{SM}}$ or $R_{\mathrm{SM}}^{\text {chir }}$ that is chiral with respect to $G_{\mathrm{SM}}$. The result is required to be precisely the following set of left-handed fermions (all fermions will be in left-handed form in this paper)

$$
3 \times\left[\left(3,2, \frac{1}{6}\right)+\left(3^{*}, 1,-\frac{2}{3}\right)+\left(3^{*}, 1, \frac{1}{3}\right)+\left(1,2,-\frac{1}{2}\right)+(1,1,1)\right]
$$

Any other particles must be non-chiral with respect to $G_{\mathrm{SM}}$. This may include left-handed anti-neutrinos in the representation $(1,0,0)$ and MSSM Higgs pairs, $\left(1,2, \frac{1}{2}\right)+\left(1,2,-\frac{1}{2}\right)$. Anything else will be called exotic.

The foregoing describes the most general configuration one could reasonably call an embedding of the standard model without chiral exotics, but we will have to impose a few additional constraints to make a search feasible. First of all we require that the standard model groups $S U(3)$ and $S U(2)$ come each from a single stack of branes, denoted a and $\mathbf{b}$ respectively. This forbids diagonal embeddings of these groups in more than one $\mathrm{CP}$ factor. In general by a stack we mean a single label for a real (orthogonal or symplectic) boundary, or a pair of conjugate labels for complex, unitary branes. The CP factor yielding $S U(3)$ must be $U(3)$, whereas the weak gauge symmetry $S U(2)$ can come from either $U(2)$ 
or $S p(2)$. The group $O(3)$ is not allowed, because one cannot get spinor representations of orthogonal groups in perturbative open string constructions.

The hypercharge generator $Y$ is a linear combination of the unitary phase factors of $U(3), U(2)$ (if available) and any other generator of one of the other factors in $G_{\mathrm{CP}}$. All representations $(3,2)$ must necessarily come from bi-fundamentals of the $\mathbf{a}$ and $\mathbf{b}$ stacks, but not all anti-quarks can come from those stacks. Although there can be anti-quarks due to chiral anti-symmetric tensors of $S U(3)$, they all have the same hypercharge. Hence there must be at least one other stack of branes, labeled $\mathbf{c}$.

In principle there could be any number of additional stacks of branes, but for purely practical reasons we allow at most one more stack (labeled $\mathbf{d}$ ) to contribute to the standard model representation $(5.1)^{3}$. Additional branes may be present, and may be required for tadpole cancellation. They will be referred to as the "hidden sector". If stack $\mathbf{d}$ does not contribute to (5.1) at all we regard it as part of the hidden sector. The standard model branes $\mathbf{a}, \mathbf{b}, \mathbf{c}$ (and $\mathbf{d}$, if present) will be called the "observable sector". Note that lefthanded anti-neutrinos ${ }^{4}$ are not listed in (5.1). We do not impose an a priori constraint on the number of left-handed anti-neutrinos, although in some cases a certain number of such states is required by anomaly cancellation in $G_{\mathrm{CP}}$. They may in fact come from the hidden sector or the observable sector, or even from strings stretching between the two sectors.

Our next condition concerns the precise definition of the standard model generator $Y$. We allow it to be embedded in the most general way possible in the Chan-Paton factors of brane $\mathbf{c}$ and $\mathbf{d}$ (in addition to the unitary phases of $\mathbf{a}$ and $\mathbf{b}$ ). In principle it could also have components in the hidden sector without affecting any of the foregoing, as long as all particles charged under those components of $Y$ are massive or at least non-chiral. One could even try to use this as a mechanism to cancel bilinear axion coupling of $Y$, which would give the $Y$-boson a mass ${ }^{5}$. We will not consider that possibility here. This is equivalent to a restriction to standard model realizations with at most four participating branes, except for one intriguing possibility: a three brane realization with a fourth brane added purely to fix the axion couplings of $Y$, without contributing to quarks or leptons. This possibility was not included in our search. It should be mentioned however, that a qualitatively similar situation does indeed arise. There are orientifold vacua where there is a U(1) arising from the SM stack of branes, under which all SM particles are neutral. In this case there is a continuous family of possible hypercharge embeddings. In some cases, the masslessness condition breaks the degeneracy. This provides a string realization of the field theory models in $[107,108,109]$. In other cases, even the masslessness condition does not lift the degeneracy.

The general form of $Y$ is

$$
Y=\sum_{\alpha} t_{\alpha} Q_{\alpha}+W_{\mathbf{c}}+W_{\mathbf{d}}
$$

\footnotetext{
${ }^{3}$ In general we also expect that the number of exotics to rise fast with the number of additional stacks participating in the SM group.

${ }^{4}$ Since our convention is to represent all matter in terms of left-handed fermions, right-handed neutrinos are referred to as left-handed anti-neutrinos.

${ }^{5}$ Anomalous U(1) masses have been calculated for general orientifolds in [13].
} 
where $\alpha$ runs over the values $\mathbf{a}, \mathbf{b}, \mathbf{c}, \mathbf{d}, Q_{\alpha}$ is the brane charge of brane $\alpha(+1$ for a complex brane, -1 for its conjugate, and 0 for a real brane), and $W_{\mathbf{c}}$ and $W_{\mathbf{d}}$ are generators from the non-abelian part of the Chan-Paton group. Therefore $W_{\mathbf{c}}$ and $W_{\mathbf{d}}$ are traceless. Such contributions to $Y$ occur for example in Pati-Salam and trinification models, and therefore we want to allow this possibility.

There is one more condition we impose for practical reasons, namely that $R_{C P}^{\text {chir }}$ may only yield representations of standard model particles or their mirrors. The main purpose of this condition (as we will see in more detail below) is to prevent an unlimited proliferation of $G_{\mathrm{CP}}$-chiral, but $G_{\mathrm{SM}}$ non-chiral representations such as $(1,1, q)+(1,1,-q)$, with $q$ arbitrary. In addition, this condition also forbids triplets of $S U(2)_{\text {weak }}$, which can be chiral with respect to $U(2)_{\mathbf{b}}$.

One may distinguish three types of matter in these models: $\mathrm{OO}, \mathrm{OH}$ and $\mathrm{HH}$, where the two letters indicate if the endpoints of the open string are in the observable or hidden sector. All conditions on $\mathrm{OO}$ matter were already formulated above. The "no chiral exotics" constraint formulated above allows $\mathrm{HH}$ matter to be chiral with respect to $G_{\mathrm{CP}}$. For $\mathrm{OH}$ matter we impose a somewhat stronger constraint, namely that there cannot be any bi-fundamentals between the standard model and the hidden sector that are chiral with respect to $G_{\mathrm{CP}}$. This is a stronger condition because the "no chiral exotics" constraint allows SM-Hidden sector bi-fundamentals as long as they are non-chiral with respect to $G_{\mathrm{SM}}$. For example a mirror quark pair $(3, V)+\left(3^{*}, V\right)$, where $V$ is a vector in a hidden sector $U(N)$ group, could be allowed under the more general rules. The resulting $U(N)$ anomalies can be cancelled in various ways.

We will allow the brane stacks $\mathbf{a}, \mathbf{b}, \mathbf{c}, \mathbf{d}$ to have identical labels, with the exception of $\mathbf{c}$ and $\mathbf{d}$ (if they are identical, we might as well regard them as a single brane stack with a larger CP multiplicity). By allowing identical labels we are able to obtain examples of unified models, such as (flipped) SU(5) or Pati-Salam like models. In the case of identical labels, we count them as follows: the QCD and weak group count as one stack each, and the branes that remain after removing the QCD and weak groups count as additional stacks, such that the total does not exceed four. For example, we can get $U(5)$ models with at most two additional CP-factors (plus any number of hidden sector branes).

We conclude this section with a summary of the kind of "exotics" (plus singlets and Higgs candidates) that may occur in generic models, indicating which kind we do and do not allow. We split $G_{\mathrm{CP}}$ into an observable and a hidden part as $G_{\mathrm{O}} \times G_{\mathrm{H}}$. In all cases we combine representations into non-chiral sets (usually, but not always pairs) if possible. We can distinguish the following possibilities

\section{Matter of type $\mathrm{OO}$}

(a) Non-chiral with respect to $G_{\mathrm{CP}}$. This may include symmetric and anti-symmetric tensors or adjoints of $S U(3)$ or of $\mathrm{SU}(2)$, mirror pairs of quarks and leptons, as well as bi-fundamentals with unusual and in a few cases even irrational charges. All particles in this class are allowed by our conditions.

(b) Chiral with respect to $G_{\mathrm{CP}}$, non-chiral with respect to $G_{S M}$. Examples are 
symmetric tensors of $U(2)_{\text {weak }}$, mirror pairs of quark and lepton doublets that are chiral with respect to $U(2)_{\text {weak }}$, mirror pairs where one member of the pair is a rank-2 tensor and the other member a bi-fundamental. We do allow such particles, except the symmetric $U(2)_{\text {weak }}$ tensors, and non-chiral pairs of quarks and leptons with non-standard charges.

(c) Chiral with respect to $G_{\mathrm{CP}}$, chiral with respect to $G_{\mathrm{SM}}$, non-chiral with respect to QED $\times$ QCD. An example of such exotics would be a fourth family. Exotics of this type are not allowed by our conditions.

(d) Chiral with respect to $G_{\mathrm{CP}}$, chiral with respect to $G_{\mathrm{SM}}$, and chiral with respect to $\mathrm{QED} \times \mathrm{QCD}$. Clearly this is not acceptable.

A mass term for exotics of type $1 \mathrm{a}$ is allowed by the full gauge symmetry, and hence it is possible that such a term is generated by shifting the moduli of the model. It is an interesting question whether the appearance of such exotics is a special feature of RCFT, or if they persist outside the rational points. It should be possible to get some insight in this question by analyzing the coupling of these particles to the moduli, but this is beyond the scope of this paper. Exotics of type $1 \mathrm{~b}$ may get a mass without invoking the standard model Higgs mechanism, and hence may become more massive than standard quarks and leptons. However, this will always require some additional dynamical mechanism beyond perturbative string theory. Exotics of type 1c require the standard model Higgs mechanism to get a mass. This may not be sufficient, since the Higgs couplings themselves may be forbidden by string symmetries, in which case additional mechanisms must be invoked. In any case it would be hard to argue that such particles would be considerably more massive than known quarks and leptons.

2. Matter of type HH. These are standard model singlets. No constraints are imposed on this kind of matter. One may distinguish two kinds.

(a) Non-chiral with respect to $G_{\mathrm{CP}}$. These particles may get a mass from continuous deformations of the model, as above.

(b) Chiral with respect to $G_{\mathrm{CP}}$, non-chiral with respect to $G_{\mathrm{H}}$. These particles may get a mass from hidden sector dynamics.

3. Matter of type $\mathrm{OH}$. In many cases particles in this class have half-integer charge. This occurs if the electromagnetic charge gets a contribution $\frac{1}{2}$ from each observable brane, which turns out to be the most frequently occurring kind of model. There are many possibilities for the chiralities, which we list here for completeness. We use a notation $\left(\chi_{G_{\mathrm{CP}}}, \chi_{G_{\mathrm{H}}}, \chi_{G_{\mathrm{O}}}, \chi_{G_{\mathrm{SM}}}, \chi_{\mathrm{QED} \times \mathrm{QCD}}\right)$, where each $\chi$ indicates chirality, and can be $Y$ (yes) or $N$ (no).
(a) $(\mathrm{N}, \mathrm{N}, \mathrm{N}, \mathrm{N}, \mathrm{N})$.
(b) $(\mathrm{Y}, \mathrm{N}, \mathrm{N}, \mathrm{N}, \mathrm{N})$.
(c) $(\mathrm{Y}, \mathrm{Y}, \mathrm{N}, \mathrm{N}, \mathrm{N})$. 
(d) $(\mathrm{Y}, \mathrm{N}, \mathrm{Y}, \mathrm{N}, \mathrm{N})$.

(e) $(\mathrm{Y}, \mathrm{N}, \mathrm{Y}, \mathrm{Y}, \mathrm{N})$.

(f) $(\mathrm{Y}, \mathrm{N}, \mathrm{Y}, \mathrm{Y}, \mathrm{Y})$.

(g) $(\mathrm{Y}, \mathrm{Y}, \mathrm{Y}, \mathrm{N}, \mathrm{N})$.

(h) $(\mathrm{Y}, \mathrm{Y}, \mathrm{Y}, \mathrm{Y}, \mathrm{N})$.

(i) $(Y, Y, Y, Y, Y)$.

An example of type $3 \mathrm{~b}$, chiral with respect to the full Chan-Paton group, but not with respect to any of its subgroups, is $(3,0, V)+\left(3^{*}, 0, V\right)+3 \times\left(1,1, V^{*}\right)+3 \times\left(1,-1, V^{*}\right)$ in $U(3) \times U(1) \times U(N)$, with the first two factors from $G_{\mathrm{O}}$ and the last from $G_{\mathrm{H}}$. Of all these possibilities, only $3 \mathrm{a}$ is allowed by our criteria. Types $3 \mathrm{~b}, 3 \mathrm{c}$ and $3 \mathrm{~g}$ might be tolerated on more general grounds, and types $3 \mathrm{f}$ and $3 \mathrm{i}$ are clearly unacceptable.

\subsection{Classification of bottom-up embeddings}

Here we will discuss the possible values of the coefficients $t_{\alpha}$ that occur in the brane decomposition of $Y$. We will use the following expression for $Y$ :

$$
Y=\sum_{\alpha} x_{\alpha} Q_{\alpha}
$$

where $Q_{\alpha}$ is the $U(1)$ charge of brane $\alpha$. In contrast to (5.2) the sum is here not a priori restricted to a definite number of branes. In our search we will allow also the possibility that diagonal Lie algebra generators $W$ of $S O(N), S p(2 N)$ or $S U(N)$ groups contribute to $Y$, but this can always be taken into account by splitting those groups into $U(m)$ factors according to the $W$ eigenvalues $e_{i}$. For example, if there are two distinct eigenvalues ${ }^{6}$ we get for symplectic groups $S p(2 N)$ a contribution $W_{\alpha}=\operatorname{diag}(N \times(e), N \times(-e))$, which can be accommodated by splitting $S p(2 N)$ into conjugate brane stacks with a CP group $U(N)$ and a contribution $e Q_{\alpha}$. Geometrically, this means that the $2 N$ symplectic branes are moved off the orientifold plane. The same reasoning applies to $O(2 N)$ branes. If there are $O(2 N+1)$ stacks, the assumption of at most two distinct eigenvalues only allows the traceless generator $W=0$ in (5.2), and hence such branes cannot contribute to $Y$ at all. Finally, $U(N)$ branes can contribute $t_{\alpha} Q_{\alpha}+\operatorname{diag}\left(n_{1} \times e_{1}, n_{2} \times e_{2}\right)$, with $n_{1}+n_{2}=$ $N, n_{1} e_{1}+n_{2} e_{2}=0$. This can be regarded as two stacks $U\left(n_{1}\right) \times U\left(n_{2}\right)$ contributing $\left(t_{\alpha}+e_{1}\right) Q_{\alpha_{1}}+\left(t_{\alpha}+e_{2}\right) Q_{\alpha_{2}}$, so that $x_{\alpha_{1}}=t_{\alpha}+e_{1}$ and $x_{\alpha_{2}}=t_{\alpha}+e_{2}$ Therefore formula 5.3 covers all cases.

The brane configurations we consider here are subject to two constraints: the spectrum must match that of the standard model in the chiral sense, with chirality defined with respect to $S U(3) \times S U(2) \times U(1)$. Furthermore all cubic anomalies in each factor of the

\footnotetext{
${ }^{6}$ Two is the maximum we allow. If there are more, this necessarily yields unconventional quark or lepton charges. For more details, see appendix A.
} 
full Chan-Paton group must cancel. This must be true because we want to be able to cancel tadpoles, and tadpole cancellation imposes cubic anomaly cancellation (mixed anomalies are cancelled by the generalized Green-Schwarz mechanism). The tadpoles are usually cancelled by adding hidden sectors, which adds new massless states to the spectrum. We do not allow these to be chiral with respect to $S U(3) \times S U(2) \times U(1)$, and hence they cannot alter the cubic anomalies. The cubic anomaly cancellation conditions that are derived from tadpole cancellation are the usual ones for the non-abelian subgroups of $U(N), N>2$. Vectors contribute 1 , symmetric tensors $N+4$ and anti-symmetric tensors $N-4$, and conjugates contribute with opposite signs. But the same condition emerges even if $N=1$ and $N=2$. This means that for example a combination of three vectors and an anti-symmetric tensor is allowed in a $U(1)$ factor. This is counter-intuitive, because the anti-symmetric tensor does not even contribute massless states, so that one is left with just three chiral massless particles, all with charge 1 . The origin of the paradox is that it is incorrect to call this condition "anomaly cancellation" if $N=1$ and $N=2$ and if chiral tensors are present. It is simply a consequence of tadpole cancellation; the anomaly introduced by the three charge 1 particles is factorizable, and cancelled by the Green-Schwarz mechanism.

One might entertain the thought that this peculiar $U(1)$ cancellation might have something to do with the fact that we have three families of standard model particles. For example, one could assign the same $U(1)$ charge to all quarks or leptons of a certain type, and then cancel this anomaly with anti-symmetric tensors. This would require this particle type to appear with a multiplicity divisible by three. Because the $U(1)$ is anomalous, it would acquire a mass via the Green-Schwarz term. However, although configurations of this kind can indeed be constructed, they are complicated and unlikely to occur. We did indeed find examples of $U(1)$ anomaly cancellations due to anti-symmetric tensors, but usually with a more complicated family structure that does not admit such an interpretation.

\subsubsection{Orientable configurations}

Let us now return to our goal of determining the possibilities for $Y$. We begin by demonstrating that in principle all real values of the leading coefficient $x_{\mathbf{a}}$ are allowed. Using the quark doublet charges we may write $Y$ as follows

$$
Y=\left(x-\frac{1}{3}\right) Q_{\mathbf{a}}+\left(x-\frac{1}{2}\right) Q_{\mathbf{b}}+\text { rest }
$$

Here we assume (without loss of generality) that the quark doublets all come from bifundamentals $\left(V, V^{*}\right)$ stretching between the QCD and the weak brane. The second entry could also be a $V$, but then we can conjugate $U(2)$ to obtain $\left(V, V^{*}\right)$. A mixture of $V$ and $V^{*}$ is however not allowed if we want $x$ to take all real values; neither is a chiral anti-symmetric tensor in either $U(3)$ or $U(2)$, or the option of using $S p(2)$ instead of $U(2)$. Here and in the following all representations are in terms of left-handed spinors.

Now we need lepton doublets. They can only be bi-fundamentals ending on the $U(2)$. The other end must be on a brane that contributes to $Y$ in such a way that the total charge 
is either $-\frac{1}{2}$ or $\frac{1}{2}$. The latter value is considered because in addition to lepton doublets, we also allow mirrors, or MSSM Higgs pairs. Again we will write these bi-fundamentals exclusively as $\left(V, V^{*}\right)$ (the first entry corresponds to $\left.U(2)\right)$. Mixtures of $(V, V)$ and $\left(V, V^{*}\right)$ between the same branes would fix $x$, and if there are no mixtures we can convert all bi-fundamentals to the form $\left(V, V^{*}\right)$. The multiplicities of these bi-fundamentals may be negative, in which case we interpret them as $\left(V^{*}, V\right)$.

Since we only allow $S U(2)$ doublets with charges $\pm \frac{1}{2}$, the possibilities for the charge coefficients of the new branes are $x$ or $x-1$. We refer to branes with these charges as "type C" and "type D" respectively (the QCD and weak branes are defined to be of type $A$ and $B$ respectively. We use small letters $\mathbf{a}, \mathbf{b}, \mathbf{c}, \mathbf{d}, \mathbf{e}, \ldots$ to label different stacks, and capitals $A, B, C, \ldots$ to label their types, with respect to the hypercharge embedding. Branes $\mathbf{a}$ and $\mathbf{b}$ are always of type $\mathrm{A}$ and $\mathrm{B}$, but there is no one-to-one correspondence for the other branes). Note that these types $\mathrm{C}$ and $\mathrm{D}$ become equivalent (up to conjugation) if and only if $x=\frac{1}{2}$. We are not requiring that the type $\mathrm{C}$ or $\mathrm{D}$ branes are identical for all leptons or Higgs, or each other's conjugate, even if their charges would allow that.

Let $n_{1}$ be the net number of chiral states between brane $\mathbf{b}$ and all of the C-type branes, and $n_{2}$ the same for type $\mathrm{D}$. To be precise:

$$
n_{1}=\sum_{i}\left[\left(N\left(V, V^{*}\right)_{\mathbf{b} C_{i}}-N\left(V^{*}, V\right)_{\mathbf{b} C_{i}}\right)\right]
$$

where $N$ is the absolute number of massless states with given properties. We now impose anomaly cancellation in $U(2)$ (for three families)

$$
-9+n_{1}+n_{2}=0
$$

because no chiral tensors are allowed for generic $x$. We also impose the requirement of having three chiral lepton doublets

$$
n_{1}-n_{2}=3
$$

which can be solved to yield $n_{1}=6$ and $n_{2}=3$. Note that the anomaly conditions for the Chan-Paton factors at the other end can aways be satisfied for some of the solutions. This is because the solution allows all multiplicities of $N\left(V, V^{*}\right)$ as well as $N\left(V^{*}, V\right)$ to be multiples of three. If we make three open strings end on the same $U(1)$ brane, the corresponding $U(1)$ anomalies can always be cancelled by anti-symmetric tensors.

Next we need anti-quarks. Since for general $x$ anti-symmetric $U(3)$ tensors are not allowed, they must be bi-fundamentals between the $U(3)$ stack and other branes. If we introduce new branes for the anti-quark strings to end on, we can always arrange the configuration so that the anti-quarks are of the form $\left(V^{*}, V\right)$. Then we need a brane of type $\mathrm{C}$ for down anti-quarks and a brane of type $\mathrm{D}$ for up anti-quarks. One may also use one of the already present branes of type $\mathrm{C}$ and $\mathrm{D}$ for this purpose, provided that only combinations $\left(V, V^{*}\right)$ or $\left(V^{*}, V\right)$ are used. Anything else implies a condition on $x$. Even if one uses distinct branes for all particle types, there are many ways to cancel the $U(1)$ anomalies using anti-symmetric tensors. 
Finally we need charged lepton singlets and their mirrors. They can occur in four different ways for generic $x$ :

1. With both ends on an existing brane of types $\mathrm{C}$ and $\mathrm{D}$.

2. With one end on a previous $\mathrm{C}$ or $\mathrm{D}$ brane and one end on a new one. This would require new branes with various possible charges. In particular, it allows the following new charges: $x+1, x-2$ and their conjugates. We refer to these as types $\mathrm{E}$ and $\mathrm{F}$. For $x=\frac{1}{2}$ these are each other's conjugates, and for $x=\frac{3}{2}, 1,0$ and $-\frac{1}{2}$ some of the types $\mathrm{C}, \mathrm{D}, \mathrm{E}$ and $\mathrm{F}$ are equivalent.

3. With both ends on the same, new brane. This requires a new brane with $t_{\alpha}= \pm \frac{1}{2}$. We call this type $G$, unless it coincides with a previous type.

4. With both ends on two distinct new branes. This would in principle allow two new branes with contributions $y$ and $1-y$ to $Y$. Such branes (if they do not coincide with any previous type) will be called type $\mathrm{H}$.

There are even more possibilities if one allows arbitrary numbers of additional branes for charged leptons. For example, one can connect new branes to types $\mathrm{E}$ and $\mathrm{F}$ with charge contributions $x-2$ or $x+3$, connect new branes to types $\mathrm{G}$ and $\mathrm{H}$ or add more branes of type $\mathrm{H}$. By allowing mirror leptons one can build arbitrarily long chains of branes in this manner. However, this is too baroque ${ }^{7}$ to consider seriously, and can in any case not be realized with at most four branes, a restriction we will ultimately impose. Already the fourth option is then impossible.

Options three and four split the standard model into two chirally disconnected sectors (i.e. there are no chiral strings connecting the two). This implies that the $Y$ anomaly does not cancel in each sector separately, and hence the two components of the would-be $Y$-boson must have Green-Schwarz couplings to axions that give it a mass. In principle these contributions could cancel for $Y$, but that seems improbable, and hence reduces the statistical likelihood of this sort of configuration in a search. Furthermore lepton Yukawa couplings are perturbatively forbidden in such models.

The same four options exist for left-handed anti-neutrinos, but we do not impose any requirements on our construction with regard to their multiplicity. If they come from strings not attached to any of the previous branes, we regard them as part of the hidden sector. Furthermore, we do not allow $Y$ to have contributions from branes that do not couple to charged quarks and leptons. Otherwise one could extend $Y$ by arbitrarily large linear combinations that only contribute non-chiral states. This implies that we regard a

\footnotetext{
${ }^{7}$ It should be kept in mind that as the number of branes participating in the SM configuration increases, the number of chiral exotics, fractionally charged particles and other unwanted states increases exponentially fast. It is possible that the lower success rate may be compensated by the potentially larger number of such configurations. It is still true however, that the effective field theory of such vacua, will be very complicated or maybe intractable.

${ }^{8}$ In the actual search we have relaxed this condition slightly, and allowed a brane $\mathbf{d}$ that just yields anti-neutrinos.
} 
brane configuration as complete (prior to tadpole cancellation) if all charged quark and leptons exist chirally, and if all cubic $U(N)$ anomalies cancel. This configuration may already contain a few candidate right-handed neutrinos, and additional ones may appear, after tadpole cancellation, from hidden sector states, or strings between the standard model and the hidden sector.

Clearly this still leaves a huge number of possibilities to realize this kind of configuration, but there is an obvious maximally economical choice, namely identifying all branes of equal charge with each other, and the brane with opposite charge with its conjugate. This then results in a $U(3) \times U(2) \times U(1) \times U(1)$ model with the following chiral spectrum

$$
\begin{aligned}
& 3 \times\left(V, V^{*}, 0,0\right) \\
& 3 \times\left(V^{*}, 0, V, 0\right) \\
& 3 \times\left(V^{*}, 0,0, V\right) \\
& 6 \times\left(0, V, V^{*}, 0\right) \\
& 3 \times\left(0, V, 0, V^{*}\right) \\
& 3 \times\left(0,0, V, V^{*}\right)
\end{aligned}
$$

Although we anticipated the possible need for anti-symmetric tensors, it turns out that they are not needed at all in this particular configuration. All anomalies are already cancelled. This is a consequence of standard model anomaly cancellation. The formula for $Y$ is

$$
Y=\left(x-\frac{1}{3}\right) Q_{\mathbf{a}}+\left(x-\frac{1}{2}\right) Q_{\mathbf{b}}+x Q_{\mathbf{c}}+(x-1) Q_{\mathbf{d}}
$$

This model has the feature that it can be realized entirely in terms of oriented strings, which of course implies that $x$ is not fixed. The converse is not true because one can allow $U(1)$ anti-symmetric tensors; they do not yield massless particles and hence give no restriction on $x$. By construction, this is the minimal realization of the standard model in terms of oriented strings. Oriented configurations (although more complicated than the one shown above) were considered earlier in [21,4] in the context of type-II theories.

One can generalize these orientable models further by allowing stack $\mathbf{c}$ and/or $\mathbf{d}$ to consist of several type $\mathrm{C}$ and $\mathrm{D}$ branes. The most general configuration can be denoted as $U(3) \times U(2) \times U\left(p_{1}+q_{1}\right) \times U\left(p_{2}+q_{2}\right)$, where $p_{1}$ is the number of type $\mathrm{C}$ branes on stack c, etc. To achieve this split we allow non-trivial generators $W_{\mathbf{c}}$ and $W_{\mathbf{d}}$ in the definition of $Y$. This gives an infinite set of solutions, all with at least three Higgs pairs (this follows from $U(2)$ anomaly cancellation). All these models have in fact precisely the same structure as the basic four-stack model above, except for an additional possibility that occurs if type $\mathrm{C}$ or $\mathrm{D}$ branes are in different positions (i.e. have different boundary labels). If in total three open strings are needed ending on brane $\mathrm{C}$ to get three anti-quarks, then if there are several type $\mathrm{C}$ branes the total number of such strings must be three. However, each multiplicity can be positive and negative, and hence cancellations are possible, that show up in the spectrum as additional mirrors on top of the basic configuration.

One of these cases corresponds to the "trinification" model [53, 1, 115, 112]. One starts with a gauge group $S U(3)_{\text {color }} \times S U(3)_{L} \times S U(3)_{R}$ and matter in three copies of the representation $\left(V, V^{*}, 0\right)+\left(V^{*}, 0, V\right)+\left(0, V, V^{*}\right)$. This configuration fits into our construction 
by starting with four stacks $(\mathbf{a}, \mathbf{b}, \mathbf{c}, \mathbf{d})$ with a CP group $U(3) \times U(2) \times U(1) \times U(3)$, and $Y=-\frac{1}{6} Q_{\mathbf{b}}+\frac{1}{3} Q_{\mathbf{c}}+W_{\mathbf{d}}$, where $W_{\mathbf{d}}$ is the $S U(3)_{\mathbf{d}}$ generator $\operatorname{diag}\left(\frac{1}{3}, \frac{1}{3},-\frac{2}{3}\right)$. The spectrum is three times $\left(V, V^{*}, 0,0\right)+\left(V, 0, V^{*}, 0\right)+\left(V^{*}, 0,0, V\right)+\left(0, V, 0, V^{*}\right)+\left(0,0, V, V^{*}\right)$. The trinification model is obtained by putting stacks $\mathbf{b}$ and $\mathbf{c}$ on top of each other. In terms of the foregoing discussion, this model has $x=\frac{1}{3}$, and three branes of type $\mathrm{C}$ (one from stack $\mathbf{c}$ and two from stack $\mathbf{d}$ ) plus one brane of type $\mathrm{D}$ (from stack $\mathbf{d}$ ). The value $x=\frac{1}{3}$ can easily be understood as follows: in a standard trinification model $Y$ is embedded entirely in $S U(3)$ factors, and cannot have components in the brane charges. Therefore in particular it cannot have any component in $U(3)_{\mathbf{a}}$.

The foregoing orientable standard model configurations can be realized in principle in non-orientable string theories. In these realizations the value of $x$ is often fixed by the requirement that $Y$ does not get a mass due to bilinear couplings with axions. Sometimes this yields rather bizarre looking solutions. For example, in our set of solutions there is one with $t_{a}=\frac{1}{33}$. There are also cases where $Y$ remains massless for any value of $x$.

\subsubsection{Charge Quantization}

There are further constraints on $x$ if one considers unoriented models. First of all, for generic values of $x$ the non-chiral part of the string spectrum contains states of fractional or even irrational charge, from $(V, V)$ bi-fundamentals or from rank-2 tensors. Since such states are always non-chiral, they may be massive, or become massive under perturbations of the model. They would however be stable and are not confined by additional gauge interactions, because they live entirely within the standard model sector. Therefore, although this possibility cannot be completely ruled out, it certainly seems preferable to avoid it.

The foregoing discussion is quite general, and can be used to analyse charge quantization for non-standard-model states in any brane realization of the standard model. The dependence on $Q_{\mathbf{a}}$ and $Q_{\mathbf{b}}$ in (5.8) is the most general one possible, up to an irrelevant sign choice. The complete string spectrum contains states with charges of all sums and differences of the components of $Y$, as well as all values multiplied by 2 . It is easy to see that just from branes $\mathbf{a}$ and $\mathbf{b}$, we get the charge quantization condition

$$
x=0 \bmod \frac{1}{2}
$$

if we require that all massive open string states from bi-fundamentals and rank two tensors between standard model branes $\mathbf{a}$ and $\mathbf{b}$ to have integer charges (taking into account QCD confinement). Clearly this condition also implies charge integrality if branes of types C,D,E and $\mathrm{F}$ are present. Only if charged leptons come from a chirally decoupled sector (the third or fourth case listed earlier) further conditions may be needed.

A second type of fractional charges that may occur are those coming from strings with a single end on a standard model brane, and the other end on a hidden sector brane. Even if these states are non-chiral, they certainly exist as massive excitations. In principle, such 
charges could be confined by hidden sector gauge groups, but to avoid them altogether, the following condition must hold

$$
x=0 \bmod 1 .
$$

Also this condition can be derived from just the $\mathbf{a}$ and $\mathbf{b}$ branes. If it is satisfied, branes of types $\mathrm{C}, \mathrm{D}, \mathrm{E}$ and $\mathrm{F}$ satisfy the hidden sector charge quantization condition, but types $\mathrm{G}$ and $\mathrm{H}$ do not, in general.

Note that the first charge quantization condition (absence of fractional charge within the standard model sector) is automatically satisfied in oriented strings for any $x$, because the strings that might violate it simply do not exist in oriented string theories. However, quantization conditions do arise if one wishes to include hidden branes. These should not contribute to $Y$. This imposes the second charge quantization condition, $x=0 \bmod 1$, for oriented strings.

\subsubsection{Non-orientable configurations}

The foregoing restrictions were necessary if one wishes to avoid non-chiral fractionally charged matter. More severe restrictions apply if some of the quarks and leptons themselves come from states that break the orientability of the open string theory.

Note first of all that in most cases both type $\mathrm{C}$ and type $\mathrm{D}$ branes are needed, in order to get up and down anti-quarks. The only way out is to get either all down anti-quarks or all up anti-quarks from anti-symmetric $U(3)$ tensors. The former possibility requires $x=\frac{1}{2}$, and then types $\mathrm{C}$ and $\mathrm{D}$ are the same. This possibility is realized in flipped $S U(5)$ models, of which we will give examples later. The second option leads to $x=0$. Then no type $\mathrm{D}$ brane is needed for the quarks, and type $\mathrm{C}$ branes do not contribute to $Y$. This possibility finds a natural realization in $S U(5)$ GUT models. For all other values of $x$ at least one type $\mathrm{C}$ and one type $\mathrm{D}$ brane is needed in addition to branes $\mathbf{a}$ and $\mathbf{b}$.

Consider now the possibility that a chiral state (a quark or lepton, or a mirror) breaks the orientability of the configuration. Obviously this sort of analysis applies to each chirally decoupled subsector separately (connected components of quiver diagrams), and we will only consider the component connected to the $\mathbf{a}$ and $\mathbf{b}$ branes.

The possibilities for such a chiral state, and the resulting restrictions on $x$ are as follows

- Chiral anti-symmetric tensors on brane $\mathbf{a} ; x=0$ or $\frac{1}{2}$

- Chiral anti-symmetric tensors on brane $\mathbf{b} ; x=0, \frac{1}{2}$ or 1

- $(V, V)$ between on branes $\mathbf{a}$ and $\mathbf{b} ; x=\frac{1}{2}$.

- Chiral tensors on a brane of type $\mathrm{C} ; x=0, \frac{1}{2}$ or $-\frac{1}{2}$

- Chiral tensors on a brane of type D; $x=\frac{3}{2}, 1$ or $\frac{1}{2}$

- $(V, V)$ between brane $\mathbf{a}$ or $\mathbf{b}$ and a type $\mathrm{C}$ brane; $x=0$ or $\frac{1}{2}$. 
- $(V, V)$ between brane $\mathbf{a}$ or $\mathbf{b}$ and a type $\mathrm{D}$ brane; $x=\frac{1}{2}$ or 1

- $(V, V)$ between type $\mathrm{C}$ and a type $\mathrm{D}$ brane; $x=0, \frac{1}{2}$ or 1

Note that the occurrence of $(V, V)$ is automatic if one of the endpoint branes is real, and that $(V, V)$ between two distinct type $\mathrm{C}$ or type $\mathrm{D}$ branes is equivalent to chiral tensors on a single such brane. We can extend this list further by including branes of types $\mathrm{E}$ and F, but this will just give similar numbers modulo half-integers. Note that in all cases the quantization condition (5.9) is satisfied.

One important general observation can be made now. For values of $x$ other than $0, \frac{1}{2}$ and 1 all quarks and lepton doublets must be realized exactly as in the orientable four-stack model discussed above, because anti-quark weak singlets can only come from bifundamentals, and $U(2)$ anomaly cancellation cannot be fixed with anti-symmetric tensors. This only leaves some freedom for the leptonic weak singlets. On the other hand, for $x=0, \frac{1}{2}$ and 1 the $U(2)$ anomaly condition can always be satisfied by adding anti-symmetric tensors. They contribute \pm 2 to the anomaly, but since the total number of doublets is even, so is the chiral number of doublets (the number of $V$ 's minus the number of $V^{*}$ ). (Note that is true for any $U(2)$ because of cancellation of global anomalies).

If we limit ourselves to four stacks, the number of possibilities is even smaller. For values of $x$ other than 0 and $\frac{1}{2}$ branes of both types $\mathrm{C}$ and $\mathrm{D}$ are needed. This means that there is no room for $\mathrm{E}$ or $\mathrm{F}$ branes and the more exotic values for $x$ they might allow. This is true even if branes $\mathrm{C}$ and $\mathrm{D}$ are "unified" into a single Chan-Paton group. In order to get a value of $x$ outside the range $-\frac{1}{2}, \ldots, \frac{3}{2}$ in a non-orientable configuration, it must be the chiral strings between the unified $\mathrm{C} / \mathrm{D}$ brane and $\mathrm{E}$ or $\mathrm{F}$ type branes that break the orientability, i.e. both $(V, V)$ and $\left(V, V^{*}\right)$ must occur. But it is easy to see that in that case such states necessarily give rise to leptons with charges \pm 2 , because they must couple to both the type $\mathrm{C}$ and the type $\mathrm{D}$ brane.

This reduces the allowed range for $x$ to $-\frac{1}{2} \ldots \frac{3}{2}$, and one can read off from the list which orientation breaking chiral states are allowed in each case. In the following sections we will show how to construct four-stack non-orientable realizations of any of these, at least as "bottom up" brane configurations.

\subsubsection{The cases $x=-\frac{1}{2}$ or $x=\frac{3}{2}$}

To get the largest and smallest numbers in this range, the only orientation breaking chiral states must be chiral tensors on a type $\mathrm{C}$ or type $\mathrm{D}$ brane, respectively. This implies that the first five representations (5.8) (those yielding quarks and lepton doublets) must be identical to those of the four-stack orientable model (up to mirror pairs due to distributing type $\mathrm{C}$ and $\mathrm{D}$ branes over various positions, as discussed above for the orientable configuration). In particular it means that we can only vary the open string origin of the charged leptons. The values $-\frac{1}{2}$ and $\frac{3}{2}$ are essentially "dual" to each other under interchange and conjugation of the type $\mathrm{C}$ and $\mathrm{D}$ branes.

To construct a non-orientable $x=-\frac{1}{2}$ configuration we start with four stacks $(\mathbf{a}, \mathbf{b}$, c, d) generating a CP group $U(3) \times U(2) \times U(1) \times U(1)$, with the latter two are type 
$\mathrm{C}$ and $\mathrm{D}$ branes respectively. The only allowed deviation in comparison to the orientable configuration are $S_{\mathbf{c}}$ symmetric tensors on brane c, $m$ bi-fundamentals $\left(V, V^{*}\right)$ between branes $\mathbf{c}$ and $\mathbf{d}, A_{\mathbf{c}}$ anti-symmetric tensors on brane $\mathbf{c}$ and $A_{\mathbf{d}}$ on brane $\mathbf{d}$. Although the anti-symmetric tensor can occur only in non-orientable strings, they do not break the orientability in the sense of fixing $x$, because they do not yield massless particles imposing constraints on $x$. Their only rôle is to cancel chiral anomalies.

We get the following conditions from cubic anomaly cancellation and the requirement that the net number of positively charged leptons must be three:

$$
\begin{aligned}
5 S_{\mathbf{c}}+m-3 A_{\mathbf{c}} & =3 \\
-m-3 A_{\mathbf{d}} & =-3 \\
m-S_{\mathbf{c}} & =3
\end{aligned}
$$

The solution is $S_{\mathbf{c}}=-3 A_{\mathbf{d}}, m=3-3 A_{\mathbf{d}}, A_{\mathbf{c}}=-6 A_{\mathbf{d}}$. Hence $m$ and $S_{\mathbf{c}}$ must be multiples of 3 , and since $S_{\mathbf{c}}=0$ brings us back to an orientable configuration, the simplest non-trivial solution is $S_{\mathbf{c}}=-3, m=0, A_{\mathbf{c}}=-6$ and $A_{\mathbf{d}}=1$. The analysis for $x=\frac{3}{2}$ is analogous, interchanging the rôles of branes $\mathrm{C}$ and $\mathrm{D}$.

Another set of possibilities (for $x=-\frac{1}{2}$ ) is obtained by putting three type-C branes in stack c, with the CP multiplicity providing the multiplicities of the anti-quarks and the lepton doublets. Now anti-symmetric tensors on brane $\mathbf{c}$ produce chiral particles, and fix $x$. A simple sequence of solutions is obtained for $S_{\mathbf{c}}=0, m=1-A_{\mathbf{d}}, A_{\mathbf{c}}=-A_{\mathbf{d}}$. This is a $U(3) \times U(2) \times U(3) \times U(1)$ solution with one anti-symmetric conjugate tensor on brane c (which provides the charged leptons) and an anti-symmetric tensor on brane $\mathbf{d}$, just to cancel anomalies.

One can generalize this further by allowing $\left(p_{1}, q_{1}\right)$ type $(\mathrm{C}, \mathrm{D})$ branes on stack $\mathbf{c}$, and $\left(p_{2}, q_{2}\right)$ type $(\mathrm{C}, \mathrm{D})$ branes. This is accomplished by having $\mathrm{CP}$ gauge groups $U\left(p_{1}+q_{1}\right)_{\mathbf{c}}$ and $U\left(p_{2}+q_{2}\right)_{\mathbf{d}}$, and splitting up their contribution to $Y$ by means of non-trivial generator $W_{\mathbf{c}}$ and $W_{\mathbf{d}}$ in (5.2). Since there must be both type C and type D branes, and they cannot come all from the same stack, we may require $p_{1}>0$ and $q_{2}>0$. Solving the constraints then yields solutions only in the following cases: $p_{1}=1$ or $3, q_{2}=0, q_{2}=1$ and arbitrary $p_{2}$, each with a sequence of allowed values for the representation multiplicities. The spectra with $p_{2} \neq 0$ are rather unappealing: they either have $G_{\mathrm{CP}}$-chiral pairs of mirror anti-quarks, or large numbers of rank-2 tensors. The ones with $p_{2}=0$ were already discussed above.

\subsubsection{The case $x=1$}

A simple way to obtain a configuration with $x=1$ is to replace the fourth CP group in the orientable configuration by $O(1)$ in order to break the orientability. In addition, there is a possibility of allowing $k$ anti-symmetric tensors of $U(2)$, yielding $k$ charged leptons. If brane $\mathbf{c}$ has a Chan-Paton group $U(1)$, the most general structure is, with CP-group 
$U(3) \times U(2) \times U(1) \times O(1)$ is

$$
\begin{aligned}
3 & \times\left(V, V^{*}, 0,0\right) \\
3 & \times\left(V^{*}, 0, V, 0\right) \\
3 & \times\left(V^{*}, 0,0, V\right) \\
m & \times\left(0, V, V^{*}, 0\right) \\
n & \times(0, V, 0, V) \\
l & \times(0,0, V, V) \\
k & \times(0, A, 0,0) \\
t & \times(0,0, A, 0)
\end{aligned}
$$

with the conditions

$$
\begin{aligned}
m-n & =3 \\
-9+m+n-2 k & =0 \\
k+l & =3 \\
9-2 m+l-3 t & =0
\end{aligned}
$$

These are respectively the requirements of three lepton doublets, $U(2)$ anomaly cancellation, three charged leptons and brane $\mathbf{c}$ anomaly cancellation. This yields a one-parameter set of solutions, $m=6+k, n=3+k, l=3-k, t=-k$. There are many more possibilities if we allow larger CP-factors for $\mathbf{c}$ and $\mathbf{d}$. It is also possible to use a $U(1)$ CP-factor for $\mathbf{d}$. This leads to an additional anomaly constraint, but there are many ways to satisfy it by replacing some of the vectors by their conjugates, and adding anti-symmetric and/or symmetric tensors. The latter yield singlet neutrinos. The complete solution is too complicated to present here.

\subsubsection{Realizations with three brane stacks for $x=0$}

The cases $x=0$ and $x=\frac{1}{2}$ allow far more possibilities. We will solve them here in general, in the special case that they are realized with just three branes, yielding a group $U(3) \times U(2) \times U(p, q)$, where $p$ and $q$ are the number of eigenvalues $x$ and $x-1$.

Consider first $x=0$. We assume that there are $t$ chiral rank-2 tensors on brane $\mathbf{a}$. Then the most general choice of bi-fundamentals for anti-quarks and lepton doublets is as follows

$$
\begin{aligned}
n & \times \\
m & \times\left(V^{*}, 0, V\right) \\
k & \times\left(0, V, V^{*}, 0, V^{*}\right) \\
l & \times(0, V, V)
\end{aligned}
$$

Furthermore we allow $r$ chiral anti-symmetric $U(2)$ tensor, and $a$ and $s$ chiral anti-symmetric and symmetric $U(p, q)$ tensors. The latter are allowed only if $q=0$ (since otherwise one 
gets charge 2 leptons), and if $q>1$ no $U(p, q)$ tensors are allowed at all. Furthermore we must require $m q=l q=0$ to prevent particles with unacceptable charges. To get three lepton doublets we need $k(p-q)=3$, i.e. $p-q= \pm 3$ or \pm 1 . The total number of charged leptons is $-r-a p q$.

Let us assume first that $q>1$. Then $a=s=0$, and $r=-3$, and $m=l=0 . U(2)$ anomaly cancellation then implies $(p+q) k-2 r-9=0$, and hence $(p+q) k=3$. But we have already seen that $k(p-q)=3$, and hence this is not consistent with the assumption. Now assume $q=1$. Also in this case $m$ and $l$ must vanish. Then the condition for getting three anti-down-quarks is $n p=3$. This allows $p=1$ or $p=3$, but neither is consistent with $p-q= \pm 3$ or \pm 1 .

Hence the only possibility is $q=0$. Then $r=-3$. The third brane does not contribute to $Y$, and the distinction between $V$ and $V^{*}$ on that brane is irrelevant for all hypercharges. The conditions for getting the right number of anti-down quarks is $(n+m) p=3$, and for lepton doublets it is $(k+l) p=3$. Hence $p$ is either 1 or 3 . Anti-up quarks can only come from the $t$ anti-symmetric $U(3)$ tensors. Hence $t=3$. In the $U(3) \times U(2)$ subgroup we find the representation $3 \times(A, 0)+3 \times\left(V, V^{*}\right)+3 \times\left(0, A^{*}\right)$, which of course fits precisely in $3 \times(10)$ of $U(5)$. The $U(1)$ generators $Y$ becomes an $S U(5)$ generator. Hence the only possibility for $x=0$ and at most three participating branes is broken $U(5)$. This can be reduced to two participating branes by putting the $\mathbf{a}$ and $\mathbf{b}$ branes on top of each other, to get unbroken $U(5)$. The $\mathrm{CP}$ group on the third brane can be $U(1)$ or $U(3)$, but since this brane does not contribute to $Y$ one can also allow $O(1)$ or $O(3)$. In that case there are no anomaly constraints to worry about. If the c-brane group is unitary, the total anomaly is $3(n-m)+2(l-k)$. This leaves many possible values, and this anomaly can be cancelled in many ways using symmetric or anti-symmetric tensors. In the spectrum, these appear as standard model singlets, i.e. candidate anti-neutrinos.

\subsubsection{Realizations with three brane stacks for $x=\frac{1}{2}$}

Consider now $x=\frac{1}{2}$. Then if $p=q$ the third brane could be orthogonal or symplectic, in which case there is no anomaly cancellation condition for it. Furthermore the weak group can then be $S p(2)$. This makes little difference, because $U(2)$ anomalies can be cancelled by means of anti-symmetric tensors, which in this case are standard model singlets (righthanded neutrinos) which we do not constrain a priori.

We assume that there are $t$ chiral rank-2 tensors on brane a. Then the most general structure is as follows

$$
\begin{aligned}
n & \times\left(V^{*}, 0, V\right) \\
m & \times\left(V^{*}, 0, V^{*}\right) \\
k & \times\left(0, V, V^{*}\right) \\
l & \times(0, V, V)
\end{aligned}
$$


We have to require

$$
\begin{aligned}
t+n p+m q & =3 \\
n q+m p & =3 \\
k p+l q-k q-l p & =3
\end{aligned}
$$

for getting the right anti-up, anti-down and lepton doublet count. The first two equations imply $(n-m)(p-q)=-t$, and the last one $(k-l)(p-q)=3$. Hence $p \neq q$, and brane c cannot be real. The only allowed values for $p-q$ are $-3,-1,1,3$, and $t$ must be a multiple of $p-q$. Given these four values, we can compute $n-m$ and $k-l$. To cancel the anomalies on brane $\mathbf{c}$ and to provide charged leptons we introduce $a$ anti-symmetric and $b$ symmetric tensors. The conditions for anomaly cancellation on brane $\mathbf{c}$, and a net number of 3 charged leptons can be combined to yield

$$
3(n-m)(p-q)-2(k-l)(p-q)-3(a-b)(p-q)=-6
$$

which together with the previous conditions implies $a-b=n-m$. The remaining equations are

$$
\begin{aligned}
(n+m)(p+q) & =6-t \\
(a+b)(p+q) & =(n-m)+2(k-l)=\frac{6-t}{p-q}
\end{aligned}
$$

From their ratio we see that $(n+m)=(p-q)(a+b)$. Furthermore we see that $p+q$ and $p-q$ must both be divisors of $6-t$. This allows a limited number of values for $p+q$, and then $(a+b)$ and $(n+m)$ are determined. Hence all solutions are specified in terms of $t$ plus a limited number of values for $p+q$ and $p-q$. There are three more parameters that are not yet specified: $k+l$, the number of anti-symmetric tensors on brane $\mathbf{b}$, and the difference between the number of $\left(V, V^{*}\right)$ and $(V, V)$ quark doublets. One linear relation between them is imposed by $U(2)$ anomaly cancellation; in the $S p(2)$ case there is no constraint.

\subsubsection{Solutions with type $\mathrm{E}$ and $\mathrm{F}$ branes}

Type $\mathrm{E}$ and $\mathrm{F}$ branes contribute to $Y$ with coefficients $x+1$ and $x-2$ respectively. They cannot contribute to to quarks or lepton doublets. We assume here that their contribution includes at least one $\left(V, V^{*}\right)$ bi-fundamental; if they produce valid quarks or lepton doublets (or mirrors) only as $(V, V)$ bi-fundamentals we conjugate the $\mathrm{E} / \mathrm{F}$ brane, and redefine its coefficients. Depending on the actual value of $x$ an $\mathrm{E}$ or $\mathrm{F}$ brane then becomes a brane of type $\mathrm{C}$ or $\mathrm{D}$, and is already included in our foregoing discussion.

Furthermore an $\mathrm{E} / \mathrm{F}$ brane must be connected, by definition, via $\left(0,0, V, V^{*}\right)$ bi-fundamentals to the c-brane. As discussed above, in a four-stack configuration $\mathrm{E}$ or $\mathrm{F}$ branes can only be allowed in principle for $x=0$ or $x=\frac{1}{2}$. As in the rest of the paper, we allow the $\mathbf{c}$ and $\mathbf{d}$ stacks to consist of two brane types, with eigenvalues differing by one unit. The options are 
then $\mathbf{c}=(\mathrm{C}, \mathrm{D}), \mathbf{d}=(\mathrm{E}, \mathrm{C})$ or $\mathbf{c}=(\mathrm{C}, \mathrm{D}), \mathbf{d}=(\mathrm{D}, \mathrm{F})$, where each type can occur with an arbitrary multiplicity, and $\mathrm{E}$ and $\mathrm{F}$ have to occur at least once. However, in all cases one of the two branes on stack $\mathbf{c}$ would give rise to a charge-2 lepton. This reduces the possibilities to $\mathbf{c}=(\mathrm{C}), \mathbf{d}=(\mathrm{E}, \mathrm{C})$ for $x=\frac{1}{2}$ (and its conjugate, $\left.\mathbf{c}=(\mathrm{D}), \mathbf{d}=(\mathrm{D}, \mathrm{F})\right)$ or $\mathbf{c}=(\mathrm{D}), \mathbf{d}=(\mathrm{D}, \mathrm{F})$ for $x=0$. However, the latter possibility is ruled out, since at least one C-type brane is needed to produce $d^{c}$ anti-quarks. The next constraint is anomaly cancellation for stack d. Since it only shares bi-fundamentals $\left(0,0, V, V^{*}\right)$ with brane $\mathbf{c}$ and nothing with any other brane, the anomalies of the $V^{*}$ 's must be cancelled by rank-2 tensors. This forbids two distinct Y-eigenvalues on stack $\mathbf{d}$, since the sums of these eigenvalues would appears as invalid charges in the spectrum. It also limits the multiplicity of the $\mathrm{E}$ or $\mathrm{F}$ branes to 1 , and only allows anti-symmetric tensors to cancel the anomaly. The multiplicity of $\left(0,0, V, V^{*}\right)$ must then be a multiple of three.

Configurations of this type can indeed be constructed. The c-group can either be $U(1)$ or $U(3)$. In the former case, there is a two-parameter series of solutions labelled by the number of $S U(3)_{\mathbf{a}}$ anti-symmetric tensors, and the number of $\left(0,0, V, V^{*}\right)$. The $U(1)_{\mathbf{c}}$ anomalies are cancelled by anti-symmetric and/or symmetric tensors, and the latter also contribute charged leptons. If c-group is $U(3)$, there must be three anti-symmetric conjugate tensors of $S U(3)_{\mathbf{a}}$ (yielding three left-handed down quarks, which must be combined with six left-handed down anti-quarks from $\left.\left(V^{*}, 0, V, 0\right)\right)$, and there can be charged leptons from $\left(0,0, V, V^{*}\right)$ as well as anti-symmetric $U(3)$ tensors.

Furthermore, one may use both $U(2)$ and $S p(2)$ as the Chan-Paton group of brane $\mathbf{b}$.

None of these models have appeared in our top-down search.

\subsubsection{Solutions with type G branes}

Type-G branes are defined as branes that contribute non-trivially to $Y$ but that contribute to the chiral spectrum only through rank-2 tensors. This implies that their $Y$-coefficient must be $\pm \frac{1}{2}$. If $x=\frac{1}{2}$, this can be viewed as just a standard type $\mathrm{C}$ or $\mathrm{D}$ brane. These cases are taken into account in our bottom-up construction as standard $x=\frac{1}{2}$ models. They do indeed occur as brane configurations, although rarely. For example, we have generated all brane configurations with four unitary $\mathrm{CP}$ factors, at most three Higgs pairs, at most three $G_{\mathrm{CP}}$ exotics and at most six $G_{\mathrm{CP}}$ chiral singlets. Of the 10820995 unitary models with $x=\frac{1}{2}$, only 338 have type-G branes, i.e. a brane with only chiral tensors and no bi-fundamentals.

A more interesting situation occurs when $x=0$ (the only other value of $x$ where type- $\mathrm{G}$ branes might occur). In that case the type- $\mathrm{G}$ brane has a non-canonical contribution $\pm \frac{1}{2}$ to $Y$ (the canonical value is 0 or \pm 1 ).

However, the foregoing analysis of three brane realizations with $x=0$ shows that this possibility does not exist. The only three-brane models are (broken) $S U(5)$ with a set of neutral C-type branes. This result was obtained without requiring any particular value for the number of charged leptons. The latter came out uniquely as three. Since the c stack is neutral, it cannot provide charged leptons or mirrors either. Hence all three-stack models already have precisely three charged leptons, and all the G-brane could still do is 
add mirror pairs. This could happen even with a chiral $\mathbf{d}$ stack, for example with three anti-symmetric tensors and a symmetric tensor of $U(2)$, with $W_{\mathbf{d}}=\operatorname{diag}\left(\frac{1}{2},-\frac{1}{2}\right)$. However, this is not of much interest, and furthermore these models are equivalent to those where brane $\mathbf{d}$ does not contribute to $Y$ at all, and brane $\mathbf{d}$ just yields $G_{\mathrm{CP}}$-chiral neutrinos.

\subsection{Top-down configurations and SM spectra}

\subsubsection{Scope of the top-down search}

The set of models we are able to search in principle consists of all three and four-stack combinations of all boundaries of all simple current orientifolds [69] of all simple current MIPFs [81][110] of the $168 c=9$ tensor products of $N=2$ minimal models. We denote these as $\left(k_{1}, \ldots, k_{m}\right)$, where $k_{i}$ is the $S U(2)$ level, which ranges from 1 to $\infty$. The total number of MIPFs is 5403, and the total number of orientifolds 49304. Some of these have zero-tension O-planes, which means that there is no possibility of cancelling tadpoles between D-branes and O-planes. This leaves 33012 orientifold models. Of the 168 Gepner models, 5 are non-chiral $K_{3} \times T_{2}$ compactifications, which need not be considered because they can never yield a chiral spectrum. These non-chiral theories contribute in total 88 MIPFs and 228 orientifolds.

The number of boundary states in a complete set can range from a few hundred to 108612 for tensor product $(1,5,82,82)$. In that case the number of unitary brane pairs is 53046 and 52920 for the two orientifold choices. The number of combinations one needs to consider for a four-stack configuration grows with the fourth power of the number of pairs. In [60] almost all these cases were searched. This was possible because the standard model configuration searched for was more limited. For example, no chiral rank-2 tensors were allowed, reducing the number of choices for the $\mathbf{a}, \mathbf{b}, \mathbf{c}$ and $\mathbf{d}$ branes dramatically. Furthermore the configuration of [98] is such that branes a and $\mathbf{d}$ have a different multiplicity (3 and 1) but identical intersection numbers with the other branes. This can be used to reduce the power behavior of the search algorithm essentially from four to three.

Neither of these shortcuts help us here, and therefore a full search is practically impossible at present. Here we limit ourselves to MIPFs with at most 1750 boundaries. This limits us to 4557 of the 5403 MIPFS and 29257 of the 33012 non-zero tension orientifolds. We can now work out how many brane configurations exist in total. To do this really correctly, unitary, orthogonal and symplectic branes must be distinguished.

Table (5.1) lists the total number of configurations for all combinations of unitary, orthogonal and symplectic branes, without taking into account the additional freedom of assigning Chan-Paton multiplicities. The second column gives the grand total for all 163

\footnotetext{
${ }^{9}$ Note that all boundaries we consider respect the full chiral algebra of the tensor product, and all partition functions are expressed in terms of the characters of that algebra, which are space-time nonchiral. One may also consider orbifold projections of these theories, which reduce the chiral algebra, and may introduce chiral characters, but our methods do not apply to that case. We do allow the inverse of this: a chiral theory with a non-chiral extension. Indeed, we found some standard model configurations for such theories.
} 
Table 5.1: Total number of three and four stack configurations of various types.

\begin{tabular}{|l|r|r|}
\hline Type & Total & This paper \\
\hline \hline UUU & 1252013821335020 & 1443610298034 \\
UUO, UOU & 99914026743414 & 230651325566 \\
UUS, USU & 14370872887312 & 184105326662 \\
USO & 2646726101668 & 74616753980 \\
USS & 1583374270144 & 73745220170 \\
UUUU & 21386252936452225944 & 366388370537778 \\
UUUO & 2579862977891650682 & 105712361839642 \\
UUUS & 187691285670685684 & 82606457831286 \\
UUOO & 148371795794926076 & 19344849644848 \\
UUOS & 17800050631824928 & 26798355134612 \\
UUSS & 4487059769514536 & 13117152729806 \\
USUU & 93838457398899186 & 41211176252312 \\
USUO & 17800050631824928 & 26798355134612 \\
USUS & 8988490411916384 & 26418410786274 \\
\hline
\end{tabular}

chiral Gepner models and non-zero tension orientifolds. It is the maximal number of three and four-stack configurations of given type that we have at our disposal for Standard Model searches. The third column gives the size of the subset actually searched in this paper.

The precise counting is as follows. Denote the number of unitary brane pairs as $N_{U}$. Then the total number of UUUU configurations with distinct $\mathbf{c}$ and $\mathbf{d}$ branes is $\left(2 N_{U}\right)\left(N_{U}\right) \times \frac{1}{2} N_{U}\left(N_{U}-1\right)$, etc. The choices for $\mathbf{a}, \mathbf{b}$ and $\mathbf{c}$ are independent, since we allow all these stacks to coincide, but if $\mathbf{c}$ and $\mathbf{d}$ coincide we regard it as a three-stack configuration. Furthermore both conjugates of the a brane are counted, because they give rise to conjugate $S U(3)$ representations, and hence yield distinct spectra. Conjugations of the $\mathbf{b}, \mathbf{c}$ and $\mathbf{d}$ branes can always be compensated by changing the sign of the coefficients of $Y$, and hence do not yield new possibilities.

Obviously, although we cover a substantial fraction of MIPFs and orientifolds, only a small fraction of possible brane configurations has been searched, because the missing MIPFs are the ones with the largest number of branes. Nevertheless, in our previous search [60], which was more extensive, the MIPFs we are not considering in the present paper produced relatively few SM-configurations and tadpole solutions. Part of the reason for the latter is that probably there are many more candidate branes in the hidden sector, making the tadpole equations harder to solve.

\subsubsection{Standard model brane configurations found}

Of the 4557 MIPFs, 1639 contained at least one standard model spectrum, without taking into account tadpole cancellation. In table (5.2) we list the total number of brane con- 
5.4 Top-down configurations and SM spectra

Table 5.2: Number of standard model configurations sorted by the value of $x$.

\begin{tabular}{|c|r|r|}
\hline$x$ & Total occurrences & Without $S U(3)$ tensors \\
\hline \hline$-1 / 2$ & 0 & 0 \\
0 & 21303612 & 202108 \\
$1 / 2$ & 124006839 & 115350426 \\
1 & 12912 & 12912 \\
$3 / 2$ & 0 & 0 \\
$*$ & 1250080 & 1250080 \\
\hline
\end{tabular}

figurations with a chiral standard model spectrum sorted according to $x$. In [60] only a subset of the possible $x=\frac{1}{2}$ models was considered, but for a much larger set of MIPFs. This produced a total of about 45 million such configurations, whereas now we find about 124 million, in both cases before attempting to solve the tadpole conditions. In column 1 , a $*$ indicates that the value of $x$ is not fixed by the quark and lepton charges, as is the case in orientable models. In these models, the value of $x$ may or may not be fixed by the zero-mass condition for $Y$. If it is fixed, it can in principle have any real value. In table (5.2) this distinction is not taken into account, but we do treat these models as distinct in the complete list, table (5.6), to be discussed below.

Apart from the $x=*$ cases, all other models are categorized with the value of $x$ that follows from the quark and lepton charges as well as the zero mass condition for $Y$. In some cases, the quark and lepton charges alone might allow more than one value of $x$ even for unorientable models. For example, in $S U(5)$ GUT models one can get the correct spectrum for $x=0$ (standard $S U(5)$ ) and $x=\frac{1}{2}$ (flipped $S U(5)$ ). The zero-mass condition for $Y$ always allows the former option (since $Y$ is a generator of the non-abelian group $S U(5)$ ) and may or may not allow the latter. If both are allowed, both are taken into account in table (5.2). Finally, if a model with $x=*$ gets $x$ fixed to a half-integer value by the $Y$-mass condition, it is counted once as an $x=*$ model, and once for the actual value of $x$.

In the third column we list how many of the configurations have no anti-quarks realized as anti-symmetric $S U(3)$ tensors. Note that anti-symmetric $S U(3)$ tensors are only allowed for $x=0$ and $x=1 / 2$. In the former case, it turns out that about $99 \%$ of the configurations have such tensors, whereas for $x=1 / 2$ only a few per cent have them.

Table 5.3: Number of standard model configurations and tadpole solutions according to type.

\begin{tabular}{|l|l|l|l|r|r|r|r|}
\hline$x$ & Config. & stack c & stack d & cases & Total occ. & Tot MIPFs & Solved \\
\hline $1 / 2$ & UUUU & C,D & C,D & 1732 & 1661111 & 8011 & $110(1,0)^{*}$ \\
\hline \multicolumn{10}{|c|}{ Continued on next page } \\
\hline
\end{tabular}


Table 5.3 - continued from previous page

\begin{tabular}{|c|c|c|c|c|c|c|c|}
\hline$x$ & Config. & stack c & stack d & cases & Total occ. & Top MIPFs & Solved \\
\hline $1 / 2$ & UUUU & $\mathrm{C}$ & $\mathrm{C}, \mathrm{D}$ & 2153 & 2087667 & 10394 & $145(43,5)^{*}$ \\
\hline $1 / 2$ & UUUU & $\mathrm{C}$ & $\mathrm{C}$ & 358 & 586940 & 1957 & $64(42,5)^{*}$ \\
\hline $1 / 2$ & UUU & $\mathrm{C}, \mathrm{D}$ & - & 2 & 28 & 2 & 0 \\
\hline $1 / 2$ & UUU & $\mathrm{C}$ & - & 7 & 13310 & 74 & $3(3,2)^{*}$ \\
\hline $1 / 2$ & UUUN & $\mathrm{C}, \mathrm{D}$ & - & 2 & 60 & 2 & 0 \\
\hline $1 / 2$ & UUUN & $\mathrm{C}$ & - & 11 & 845 & 28 & 0 \\
\hline $1 / 2$ & UUUR & $\mathrm{C}, \mathrm{D}$ & C,D & 1361 & 3242251 & 12107 & $128(1,0)^{*}$ \\
\hline $1 / 2$ & UUUR & $\mathrm{C}$ & C,D & 914 & 3697145 & 12294 & $105(72,6)^{*}$ \\
\hline $1 / 2$ & USUU & $\mathrm{C}, \mathrm{D}$ & C,D & 1760 & 4138505 & 14829 & $70(2,0)^{*}$ \\
\hline $1 / 2$ & USUU & $\mathrm{C}$ & $\mathrm{C}, \mathrm{D}$ & 1763 & 8232083 & 17928 & $163(47,5)^{*}$ \\
\hline $1 / 2$ & USUU & $\mathrm{C}$ & $\mathrm{C}$ & 201 & 4491695 & 3155 & $48(39,7)^{*}$ \\
\hline $1 / 2$ & USU & $\mathrm{C}, \mathrm{D}$ & - & 5 & 13515 & 384 & $5(2,0)$ \\
\hline $1 / 2$ & USU & $\mathrm{C}$ & - & 2 & 222 & 4 & 0 \\
\hline $1 / 2$ & USUN & $\mathrm{C}, \mathrm{D}$ & - & 29 & 46011 & 338 & $2(2,0)$ \\
\hline $1 / 2$ & USUN & $\mathrm{C}$ & - & 1 & 32 & 1 & 0 \\
\hline $1 / 2$ & USUR & C,D & C,D & 944 & 45877435 & 34233 & $130(4,0)^{*}$ \\
\hline $1 / 2$ & USUR & $\mathrm{C}$ & C,D & 207 & 49917984 & 11722 & $70(54,10)^{*}$ \\
\hline 0 & UUUU & $\mathrm{C}, \mathrm{D}$ & C,D & 20 & 7950 & 110 & $2(2,0)$ \\
\hline 0 & UUUU & $\mathrm{C}$ & $\mathrm{C}, \mathrm{D}$ & 164 & 50043 & 557 & $8(0,0)$ \\
\hline 0 & UUUU & D & $\mathrm{C}, \mathrm{D}$ & 5 & 4512 & 40 & 0 \\
\hline 0 & UUUU & $\mathrm{C}$ & $\mathrm{C}$ & 1459 & 999122 & 5621 & $119(40,3)^{*}$ \\
\hline 0 & UUUU & $\mathrm{C}$ & D & 26 & 6830 & 54 & 0 \\
\hline 0 & UUU & $\mathrm{C}$ & - & 11 & 17795 & 225 & $3(3,3)^{*}$ \\
\hline 0 & UUUN & $\mathrm{C}$ & - & 31 & 5989 & 133 & 0 \\
\hline 0 & UUUR & $\mathrm{C}, \mathrm{D}$ & $\mathrm{C}$ & 90 & 195638 & 702 & $4(4,0)$ \\
\hline 0 & UUUR & $\mathrm{C}$ & $\mathrm{C}$ & 4411 & 7394459 & 24715 & $392(112,2)^{*}$ \\
\hline 0 & UUUR & $\mathrm{D}$ & $\mathrm{C}$ & 24 & 50752 & 148 & 0 \\
\hline 0 & UUR & $\mathrm{C}$ & - & 8 & 233071 & 1222 & $6(6,0)$ \\
\hline 0 & UURN & $\mathrm{C}$ & - & 37 & 260450 & 654 & $4(4,0)$ \\
\hline 0 & UURR & $\mathrm{C}$ & $\mathrm{C}$ & 1440 & 12077001 & 15029 & $218(44,0)$ \\
\hline 1 & UUUU & $\mathrm{C}, \mathrm{D}$ & C,D & 5 & 212 & 8 & 0 \\
\hline 1 & UUUU & $\mathrm{C}$ & C,D & 6 & 7708 & 21 & 0 \\
\hline 1 & UUUU & $\mathrm{D}$ & $\mathrm{C}, \mathrm{D}$ & 4 & 7708 & 11 & 0 \\
\hline 1 & UUUR & $\mathrm{C}, \mathrm{D}$ & $\mathrm{D}$ & 1 & 1024 & 2 & 0 \\
\hline 1 & UUUR & $\mathrm{C}$ & $\mathrm{D}$ & 1 & 640 & 4 & 0 \\
\hline$*$ & UUUU & $\mathrm{C}, \mathrm{D}$ & $\mathrm{C}, \mathrm{D}$ & 109 & 571472 & 1842 & $19(1,0)^{*}$ \\
\hline * & UUUU & $\mathrm{C}$ & $\mathrm{C}, \mathrm{D}$ & 32 & 521372 & 1199 & $7(7,0)$ \\
\hline$*$ & UUUU & $\mathrm{D}$ & $\mathrm{C}, \mathrm{D}$ & 8 & 157232 & 464 & 0 \\
\hline$*$ & UUUU & $\mathrm{C}$ & D & 1 & 4 & 1 & 0 \\
\hline
\end{tabular}


Table 5.3 summarizes all 19345 top-down distinct spectra we have observed after considering all three and four stacks counted in the last column of table (5.1). The spectra are distinguished on the basis of the chiral numbers of rank-2 tensors and bi-fundamentals, the decomposition of $Y$, the presence and embedding of additional massless (i.e. not acquiring mass from axion couplings) $U(1)$-gauge bosons from the $\mathbf{a}, \mathbf{b}, \mathbf{c}, \mathbf{d}$ stacks and brane unification among the $\mathbf{a}, \mathbf{b}, \mathbf{c}, \mathbf{d}$ branes. The columns contain the following data:

- 1. The value of $x$. An asterisk indicates that any value is allowed. In all other cases the value of $x$ is the one determined from the "zero $Y$-mass" condition.

- 2. Number of participating branes and their property:

- U: Unitary (complex)

- S: Symplectic

- R: Real (Symplectic or Orthogonal)

- N: Neutral (see below for a definition)

- 3. Composition of stack $\mathbf{c}$ in terms of branes of types $\mathrm{C}$ and D.

- 4. Composition of stack $\mathbf{d}$ in terms of branes of types $\mathrm{C}$ and $\mathrm{D}$.

- 5. Total number of distinct (in the sense defined above) spectra of the type specified in the first four columns.

- 6. Total number of spectra of given type. This is the grand total of all such spectra found after scanning all the three and four brane configurations in the last column of table (5.1), and assigning Chan-Paton multiplicities in order to get the Standard Model gauge group and spectrum.

- 7. Total number of MIPFs for which spectra of given type were found.

- 8. Number of distinct spectra for which tadpole solutions were found. Between parenthesis we specify how may of these solutions have at most three mirror pairs, three MSSM Higgs pairs and six singlet neutrinos, and how many have no mirror pairs, at most one Higgs pairs, and precisely three singlet neutrinos. An asterisk indicates that at least one solution was found without additional hidden branes.

In column 2, "Neutral" means that this brane does not participate to $\mathrm{Y}$, and that there are no chiral bi-fundamentals ending on it. The latter fact implies that there must be chiral rank-2 tensors in this brane (which in particular implies that it must be unitary), or otherwise it would violate condition $5 \mathrm{~b}$ of the search algorithm (see 5.4.6). Such a brane can only give singlet neutrinos. We found a total of 111 such cases. They are anomaly free by having (a multiple of) $-(N-4)$ symmetric tensors and $(N+4)$ antisymmetric ones (for $N=4$ the anti-symmetric tensors are actually real, and should strictly speaking have been omitted.) An N-brane can always be removed to get a valid three-stack model, which of 
course satisfies all our search criteria by itself. Note that branes of this kind are in any case allowed to exist in the hidden sector, and therefore from the point of view of classification it is most natural to view these models as three-stack models with one additional hidden sector brane. The reason we explicitly allowed them is that singlet neutrinos from separate branes might be of interest for understanding the neutrino mass problem. In the following analysis we will omit these 111 cases.

\subsubsection{Bottom-up versus Top-down}

In table( 5.4) and (5.5) we compare the bottom-up and top-down results. This can only be done by imposing some restrictions on the spectra. In addition to three families of quarks and leptons and fully non-chiral matter (which we ignore) there can be $G_{C P}$-chiral matter that is $G_{S M}$ non-chiral. The possibilities are mirror pairs of fermions, singlet neutrino's and MSSM Higgs pairs. Denote these three quantities as $M, N$ and $H$. If we leave them unrestricted, there is an infinite number of bottom up solutions. Given the current experimental knowledge, the optimal values for getting the Standard Model would appear to be $M=0, N=3$ and $H=1$. However, if there is a surplus of these particles, one can assume that they get a standard-model-allowed mass above the weak scale. On the other hand, if there is a shortage $(H=0$ or $N<3)$, there still remains a possibility that the missing particles can come from $G_{C P}$ non-chiral matter, or (in the case of neutrinos) from additional branes (other than $\mathbf{a}, \mathbf{b}, \mathbf{c}$ or $\mathbf{d}$ ). Note for example that most of the models of [60] have no $G_{C P}$-chiral Higgses, but usually a large number of fully non-chiral Higgs candidates. Since we have to impose cuts on $M, N$ and $H$ to make the comparison, we present the comparison for two cases: a loose cut (with $M \leq 3, N \leq 6, H \leq 3$ ) and a tight cut $(M=0, H \leq 1$ and $N=3)$. The former comparison is in table (5.4) and the latter in table (5.5). In both tables, the number of bottom-up configurations satisfying the criteria is listed in column 5. In column 6 , we list the number of those bottom-up configurations that was encountered in our search, and in column 7 the total number of occurrences of the given class ${ }^{10}$ of configurations, summed over all three or four brane combination considered in the search. This is the same information as in column 6 of table (5.3), but with the limit on the numbers $M, N$ and $H$ imposed. In column 8 we list the number of distinct configurations for which the tadpole conditions were solved. In these tables the top-down spectra are only distinguished on the basis of criteria that can be directly compared to the bottom-up approach. Brane unification is ignored and the masses of $U(1)$ vector bosons are not taken into account. This means that some models that were distinct in the previous table are considered identical here, because they merely differ by branes that are not on top of each other, or by different embeddings of an additional massless $U(1)$ factor. This affects column 6 and column 8 , but not column 7 , which is simply the sum of all occurrences within the class. Note for example in the class $(x=*$, UUUU, $\mathbf{c}=\mathrm{C}, \mathbf{d}=(\mathrm{C}, \mathrm{D}))$ there is a total number of occurrences of 521372 in both tables. This implies that all models satisfy the constraints on the number of Higgs, mirrors and neutrinos. In table 5.1 these models

\footnotetext{
${ }^{10}$ By "class" we mean here all brane configurations that match the criteria in the first four columns.
} 
correspond to 32 distinct cases with 7 distinct solutions, whereas in table 5.4 they form only 7 distinct models with 3 distinct solutions.

Table 5.4: Bottom-up versus Top-down results for spectra with at most three mirror pairs, at most three MSSM Higgs pairs, and at most six singlet neutrinos.

\begin{tabular}{|c|c|c|c|c|c|c|c|}
\hline$x$ & Config. & stack $\mathbf{c}$ & stack $\mathbf{d}$ & Bottom-up & Top-down & Occurrences & Solved \\
\hline $1 / 2$ & $\overline{\mathrm{UUUU}}$ & $\overline{C, D}$ & $\mathrm{C}, \mathrm{D}$ & 27 & 9 & 5194 & 1 \\
\hline $1 / 2$ & UUUU & $\mathrm{C}$ & C,D & 103441 & 434 & 1056708 & 31 \\
\hline $1 / 2$ & UUUU & $\mathrm{C}$ & $\mathrm{C}$ & 10717308 & 156 & 428799 & 24 \\
\hline $1 / 2$ & UUUU & $\mathrm{C}$ & $\mathrm{F}$ & 351 & 0 & 0 & 0 \\
\hline $1 / 2$ & UUU & $\mathrm{C}, \mathrm{D}$ & - & 4 & 1 & 24 & 0 \\
\hline $1 / 2$ & UUU & $\mathrm{C}$ & - & 215 & 5 & 13310 & 2 \\
\hline $1 / 2$ & UUUR & $\mathrm{C}, \mathrm{D}$ & C,D & 34 & 5 & 3888 & 1 \\
\hline $1 / 2$ & UUUR & $\mathrm{C}$ & C,D & 185520 & 221 & 2560681 & 31 \\
\hline $1 / 2$ & USUU & $\mathrm{C}, \mathrm{D}$ & C,D & 72 & 7 & 6473 & 2 \\
\hline $1 / 2$ & USUU & $\mathrm{C}$ & C,D & 153436 & 283 & 3420508 & 33 \\
\hline $1 / 2$ & USUU & $\mathrm{C}$ & $\mathrm{C}$ & 10441784 & 125 & 4464095 & 27 \\
\hline $1 / 2$ & USUU & $\mathrm{C}$ & $\mathrm{F}$ & 184 & 0 & 0 & 0 \\
\hline $1 / 2$ & USU & $\mathrm{C}$ & - & 104 & 2 & 222 & 0 \\
\hline $1 / 2$ & USU & $\mathrm{C}, \mathrm{D}$ & - & 8 & 1 & 4881 & 1 \\
\hline $1 / 2$ & USUR & $\mathrm{C}$ & C,D & 54274 & 31 & 49859327 & 19 \\
\hline $1 / 2$ & USUR & $\mathrm{C}, \mathrm{D}$ & C,D & 36 & 2 & 858330 & 2 \\
\hline 0 & UUUU & $\mathrm{C}, \mathrm{D}$ & C,D & 5 & 5 & 4530 & 2 \\
\hline 0 & UUUU & $\mathrm{C}$ & C,D & 8355 & 44 & 54102 & 2 \\
\hline 0 & UUUU & $\mathrm{D}$ & C,D & 14 & 2 & 4368 & 0 \\
\hline 0 & UUUU & $\mathrm{C}$ & $\mathrm{C}$ & 2890537 & 127 & 666631 & 9 \\
\hline 0 & UUUU & $\mathrm{C}$ & $\mathrm{D}$ & 36304 & 16 & 6687 & 0 \\
\hline 0 & UUU & $\mathrm{C}$ & - & 222 & 2 & 15440 & 1 \\
\hline 0 & UUUR & C,D & $\mathrm{C}$ & 3702 & 39 & 171485 & 4 \\
\hline 0 & UUUR & $\mathrm{C}$ & $\mathrm{C}$ & 5161452 & 289 & 4467147 & 32 \\
\hline 0 & UUUR & $\mathrm{D}$ & $\mathrm{C}$ & 8564 & 22 & 50748 & 0 \\
\hline 0 & UUR & $\mathrm{C}$ & - & 58 & 2 & 233071 & 2 \\
\hline 0 & UURR & $\mathrm{C}$ & $\mathrm{C}$ & 24091 & 17 & 8452983 & 17 \\
\hline 1 & UUUU & $\mathrm{C}, \mathrm{D}$ & C,D & 4 & 1 & 1144 & 1 \\
\hline 1 & UUUU & $\mathrm{C}$ & C,D & 16 & 5 & 10714 & 0 \\
\hline 1 & UUUU & $\mathrm{D}$ & C,D & 42 & 3 & 3328 & 0 \\
\hline 1 & UUUU & $\mathrm{C}$ & $\mathrm{D}$ & 870 & 0 & 0 & 0 \\
\hline 1 & UUUR & C,D & $\mathrm{D}$ & 34 & 1 & 1024 & 0 \\
\hline 1 & UUUR & $\mathrm{C}$ & $\mathrm{D}$ & 609 & 1 & 640 & 0 \\
\hline $3 / 2$ & UUUU & $\mathrm{C}$ & $\mathrm{D}$ & 9 & 0 & 0 & 0 \\
\hline
\end{tabular}


Table 5.4 - continued from previous page

\begin{tabular}{|c|l|l|l|r|r|r|r|}
\hline$x$ & Config. & stack c & stack d & Bottom-up & Top-down & Occurrences & Solved \\
\hline $3 / 2$ & UUUU & C,D & D & 1 & 0 & 0 & 0 \\
$3 / 2$ & UUUU & C, D & C & 10 & 0 & 0 & 0 \\
$3 / 2$ & UUUU & C,D & C,D & 2 & 0 & 0 & 0 \\
$*$ & UUUU & C,D & C,D & 2 & 2 & 5146 & 1 \\
$*$ & UUUU & C & C,D & 10 & 7 & 521372 & 3 \\
$*$ & UUUU & D & C,D & 1 & 1 & 116 & 0 \\
$*$ & UUUU & C & D & 3 & 1 & 4 & 0 \\
\hline \hline
\end{tabular}

Some bottom-up solutions can exist for more than one value of $Y$. The most obvious example is the class $x=*$, which can exist for all values of $Y$. In making the comparison we have used the actual massless linear combination of $Y$ allowed by the axion-gauge boson couplings in the top-down Gepner model. Only for the $x=*$ case we have ignored the precise form of $Y$, because this would split this class into an indefinite number of subclasses. However, in those cases where $Y$ was of the form corresponding to $x=0, \frac{1}{2}$ or 1 , we have compared those top-down models twice: once in the $x=*$ class, and once in the class given by $Y$. This explains the tadpole solution indicated in the last column of table (5.4) for an $x=1$ model. Actually, this model has $x=*$, but $x$ is fixed to 1 by the $Y$-mass condition.

Table 5.5: Bottom-up versus Top-down results for spectra without mirror pairs, at most one MSSM Higgs pair, and precisely three singlet neutrinos. Only cases that have been found in the top-dow search are shown.

\begin{tabular}{|l|l|l|l|r|r|r|r|}
\hline$x$ & Config. & stack c & stack d & Bottom-up & Top-down & Occurrences & Solved \\
\hline $1 / 2$ & UUU & $\mathrm{C}$ & - & 8 & 2 & 13242 & 1 \\
$1 / 2$ & UUUU & $\mathrm{C}$ & $\mathrm{C}$ & 10670 & 16 & 81985 & 4 \\
$1 / 2$ & UUUU & $\mathrm{C}$ & $\mathrm{C}, \mathrm{D}$ & 148 & 8 & 378418 & 3 \\
$1 / 2$ & UUUR & $\mathrm{C}$ & $\mathrm{C}, \mathrm{D}$ & 495 & 13 & 641485 & 3 \\
$1 / 2$ & USUU & $\mathrm{C}$ & $\mathrm{C}, \mathrm{D}$ & 314 & 6 & 2757164 & 3 \\
$1 / 2$ & USUU & $\mathrm{C}$ & $\mathrm{C}$ & 10816 & 6 & 4037872 & 4 \\
$1 / 2$ & USUR & $\mathrm{C}$ & $\mathrm{C}, \mathrm{D}$ & 434 & 3 & 47689675 & 3 \\
0 & UUUU & $\mathrm{C}$ & $\mathrm{C}, \mathrm{D}$ & 23 & 1 & 6 & 0 \\
0 & UUUU & $\mathrm{C}$ & $\mathrm{C}$ & 1996 & 5 & 17301 & 2 \\
0 & UUUU & $\mathrm{C}$ & $\mathrm{D}$ & 91 & 4 & 4227 & 0 \\
0 & UUU & $\mathrm{C}$ & - & 9 & 1 & 15282 & 1 \\
0 & UUUR & $\mathrm{C}$ & $\mathrm{C}$ & 5136 & 15 & 63051 & 1 \\
\hline \hline
\end{tabular}


Table (5.6) contains all 19345 distinct models we found. Unfortunately the full table would be more than 500 pages, and is too long to include, so we have only displayed the top and some entries of interest. ${ }^{11}$ The table is ordered according to the total number of occurrences (listed in column 2) of a given spectrum. Column 3 gives the number of MIPFs for which it occurs. This gives some more indication how rare a certain spectrum is. In column 4 we give the Chan-Paton group, with factors combined if some of the branes are on the same position. In column 5 we give a rough indication of the spectrum. Here "V" means that a CP-factor only contributes bi-fundamentals, "S" "A") that there is at least one (anti)-symmetric tensor and "T" that both occur. Column 6 gives the value of $x$, and the last column indicates if a solution to the tadpole conditions was found ("Y"), and if a solution was found without additional branes ("Y!").

Table 5.6: The list of 19345 models sorted according to frequency

\begin{tabular}{|c|c|c|c|c|c|c|}
\hline $\mathrm{Nr}$ & Total occ. & MIPFs & Chan-Paton Group & Spectrum & $\mathrm{x}$ & Solved \\
\hline 1 & 9801844 & 648 & $U(3) \times S p(2) \times S p(6) \times U(1)$ & VVVV & $1 / 2$ & $\mathrm{Y} !$ \\
\hline 2 & $8479808(16227372)$ & 675 & $U(3) \times S p(2) \times S p(2) \times U(1)$ & VVVV & $1 / 2$ & $\mathrm{Y} !$ \\
\hline 3 & 5775296 & 821 & $U(4) \times S p(2) \times S p(6)$ & VVV & $1 / 2$ & $\mathrm{Y} !$ \\
\hline 4 & 4810698 & 868 & $U(4) \times S p(2) \times S p(2)$ & VVV & $1 / 2$ & $\mathrm{Y} !$ \\
\hline 5 & 4751603 & 554 & $U(3) \times S p(2) \times O(6) \times U(1)$ & VVVV & $1 / 2$ & $\mathrm{Y} !$ \\
\hline 6 & 4584392 & 751 & $U(4) \times S p(2) \times O(6)$ & VVV & $1 / 2$ & $\mathrm{Y}$ \\
\hline 7 & $4509752(9474494)$ & 513 & $U(3) \times S p(2) \times O(2) \times U(1)$ & VVVV & $1 / 2$ & $\mathrm{Y} !$ \\
\hline 8 & 3744864 & 690 & $U(4) \times S p(2) \times O(2)$ & VVV & $1 / 2$ & $\mathrm{Y} !$ \\
\hline 9 & 3606292 & 467 & $U(3) \times S p(2) \times S p(6) \times U(3)$ & VVVV & $1 / 2$ & $\mathrm{Y}$ \\
\hline 10 & 3093933 & 623 & $U(6) \times S p(2) \times S p(6)$ & VVV & $1 / 2$ & Y \\
\hline 11 & 2717632 & 461 & $U(3) \times S p(2) \times S p(2) \times U(3)$ & VVVV & $1 / 2$ & $\mathrm{Y} !$ \\
\hline 12 & 2384626 & 560 & $U(6) \times S p(2) \times O(6)$ & VVV & $1 / 2$ & $\mathrm{Y}$ \\
\hline 13 & 2253928 & 669 & $U(6) \times S p(2) \times S p(2)$ & VVV & $1 / 2$ & $\mathrm{Y} !$ \\
\hline 14 & 1803909 & 519 & $U(6) \times S p(2) \times O(2)$ & VVV & $1 / 2$ & $\mathrm{Y} !$ \\
\hline 15 & 1676493 & 517 & $U(8) \times S p(2) \times S p(6)$ & VVV & $1 / 2$ & $\mathrm{Y}$ \\
\hline 16 & 1674416 & 384 & $U(3) \times S p(2) \times O(6) \times U(3)$ & VVVV & $1 / 2$ & $\mathrm{Y}$ \\
\hline 17 & 1654086 & 340 & $U(3) \times S p(2) \times U(3) \times U(1)$ & VVVV & $1 / 2$ & $\mathrm{Y}$ \\
\hline 18 & 1654086 & 340 & $U(3) \times S p(2) \times U(3) \times U(1)$ & VVVV & $1 / 2$ & Y \\
\hline 19 & 1642669 & 360 & $U(3) \times S p(2) \times S p(6) \times U(5)$ & VVVV & $1 / 2$ & $\mathrm{Y}$ \\
\hline 20 & 1486664 & 346 & $U(3) \times S p(2) \times O(2) \times U(3)$ & VVVV & $1 / 2$ & $\mathrm{Y} !$ \\
\hline 21 & 1323363 & 476 & $U(8) \times S p(2) \times O(6)$ & VVV & $1 / 2$ & Y \\
\hline 22 & 1135702 & 350 & $U(3) \times S p(2) \times S p(2) \times U(5)$ & VVVV & $1 / 2$ & $\mathrm{Y} !$ \\
\hline 23 & 1050764 & 532 & $U(8) \times S p(2) \times S p(2)$ & VVV & $1 / 2$ & Y \\
\hline 24 & 956980 & 421 & $U(8) \times S p(2) \times O(2)$ & VVV & $1 / 2$ & $\mathrm{Y}$ \\
\hline 25 & 950003 & 449 & $U(10) \times S p(2) \times S p(6)$ & VVV & $1 / 2$ & $\mathrm{Y}$ \\
\hline 26 & 910132 & 51 & $U(3) \times U(2) \times S p(2) \times O(1)$ & AAVV & 0 & Y \\
\hline$\ldots$ & & & & & & \\
\hline
\end{tabular}

\footnotetext{
${ }^{11}$ However, the full list is available on request.
} 
Table 5.6 - continued from previous page

\begin{tabular}{|c|c|c|c|c|c|c|}
\hline $\mathrm{nr}$ & Total occ. & MIPFs & Chan-Paton Group & Spectrum & $\mathrm{x}$ & Solved \\
\hline 34 & $869428(1096682)$ & 246 & $U(3) \times S p(2) \times U(1) \times U(1)$ & VVVV & $1 / 2$ & $\mathrm{Y} !$ \\
\hline 153 & 115466 & 335 & $U(4) \times U(2) \times U(2)$ & VVV & $1 / 2$ & Y \\
\hline 225 & 71328 & 167 & $U(3) \times U(3) \times U(3)$ & VVV & $1 / 3$ & \\
\hline 303 & 47664 & 18 & $U(3) \times U(2) \times U(1) \times U(1)$ & AAVA & $1 / 2$ & Y \\
\hline 304 & 47664 & 18 & $U(3) \times U(2) \times U(1) \times U(1)$ & AAVA & 0 & Y \\
\hline 343 & 40922(49794) & 63 & $U(3) \times S p(2) \times U(1) \times U(1)$ & VVVV & $1 / 2$ & $\mathrm{Y} !$ \\
\hline 411 & 31000 & 17 & $U(3) \times U(2) \times U(1) \times U(1)$ & AAVA & 0 & $\mathrm{Y}$ \\
\hline 417 & 30396 & 26 & $U(3) \times U(2) \times U(1) \times U(1)$ & AAVS & 0 & Y \\
\hline 495 & 23544 & 14 & $U(3) \times U(2) \times U(1) \times U(1)$ & AAVS & 0 & \\
\hline 509 & 22156 & 17 & $U(3) \times U(2) \times U(1) \times U(1)$ & AAVS & 0 & Y \\
\hline 519 & 21468 & 13 & $U(3) \times U(2) \times U(1) \times U(1)$ & AAVA & 0 & Y \\
\hline 543 & $20176(*)$ & 38 & $U(3) \times U(2) \times U(1) \times U(1)$ & VVVV & $1 / 2$ & Y \\
\hline 617 & 16845 & 296 & $U(5) \times O(1)$ & AV & 0 & Y \\
\hline 671 & $14744(*)$ & 29 & $U(3) \times U(2) \times U(1) \times U(1)$ & VVVV & $1 / 2$ & \\
\hline 761 & 12067 & 26 & $U(3) \times U(2) \times U(1)$ & AAS & $1 / 2$ & $\mathrm{Y} !$ \\
\hline 762 & 12067 & 26 & $U(3) \times U(2) \times U(1)$ & AAS & 0 & $\mathrm{Y} !$ \\
\hline 1024 & 7466 & 7 & $U(3) \times U(2) \times U(2) \times U(1)$ & VAAV & 1 & \\
\hline 1125 & 6432 & 87 & $U(3) \times U(3) \times U(3)$ & VVV & $*$ & Y \\
\hline 1201 & $5764(*)$ & 20 & $U(3) \times U(2) \times U(1) \times U(1)$ & VVVV & $1 / 2$ & \\
\hline 1356 & $5856\left(^{*}\right)$ & 10 & $U(3) \times U(2) \times U(1) \times U(1)$ & VVVV & $1 / 2$ & Y \\
\hline 1725 & 2864 & 14 & $U(3) \times U(2) \times U(1) \times U(1)$ & VVVV & $1 / 2$ & Y \\
\hline 1886 & 2381 & 115 & $U(6) \times S p(2)$ & AV & $1 / 2$ & $\mathrm{Y} !$ \\
\hline 1887 & 2381 & 115 & $U(6) \times S p(2)$ & AV & 0 & $\mathrm{Y} !$ \\
\hline 1888 & 2381 & 115 & $U(6) \times S p(2)$ & $\mathrm{AV}$ & $1 / 2$ & $\mathrm{Y} !$ \\
\hline 2624 & 1248 & 3 & $U(3) \times U(2) \times U(2) \times U(3)$ & VAAV & 1 & \\
\hline 2880 & 1049 & 34 & $U(5) \times U(1)$ & AS & $1 / 2$ & $\mathrm{Y} !$ \\
\hline 2881 & 1049 & 34 & $U(5) \times U(1)$ & AS & 0 & $\mathrm{Y} !$ \\
\hline 2807 & $1096\left(^{*}\right)$ & 8 & $U(3) \times U(2) \times U(1) \times U(1)$ & VVVV & $1 / 2$ & \\
\hline 2919 & 1024 & 2 & $U(3) \times U(2) \times U(2) \times O(3)$ & VAAV & 1 & \\
\hline 4485 & $400(*)$ & 2 & $U(3) \times U(2) \times U(1) \times U(1)$ & VVVV & $1 / 2$ & \\
\hline 4727 & 352 & 3 & $U(3) \times U(2) \times U(1) \times U(1)$ & VVVV & $1 / 2$ & \\
\hline 4825 & 332 & 20 & $U(4) \times U(2) \times U(2)$ & VAS & $1 / 2$ & $\mathrm{Y} !$ \\
\hline 4902 & $320(*)$ & 1 & $U(3) \times U(2) \times U(1) \times U(1)$ & VVVV & $1 / 2$ & $\mathrm{Y}$ \\
\hline 4996 & 304 & 30 & $U(3) \times S p(2) \times U(1) \times U(1)$ & VVVV & $1 / 2$ & Y \\
\hline 6993 & $128(* *)$ & 1 & $U(3) \times U(2) \times U(2) \times U(1)$ & VVVV & $1 / 2$ & \\
\hline 7053 & 124 & 4 & $U(3) \times U(2) \times U(2) \times U(1)$ & VASV & $1 / 2$ & $\mathrm{Y} !$ \\
\hline 7241 & $116(* *)$ & 4 & $U(3) \times U(2) \times U(2) \times U(1)$ & VVVV & $1 / 2$ & \\
\hline 7280 & 114 & 3 & $U(3) \times S p(2) \times U(1)$ & AVS & $1 / 2$ & \\
\hline 7464 & 108 & 1 & $U(3) \times S p(2) \times U(1)$ & VVT & $1 / 2$ & \\
\hline 7905 & $96(*)$ & 1 & $U(3) \times U(2) \times U(1) \times U(1)$ & VVVV & $1 / 2$ & \\
\hline 8747 & $68(* *)$ & 3 & $U(3) \times U(2) \times U(1) \times U(1)$ & VVVV & $1 / 2$ & \\
\hline 8773 & 68 & 4 & $U(3) \times U(2) \times U(1) \times U(1)$ & VVVV & $1 / 2$ & \\
\hline
\end{tabular}


5.4 Top-down configurations and SM spectra

Table 5.6 - continued from previous page

\begin{tabular}{|c|l|l|l|r|l|l|}
\hline $\mathrm{nr}$ & Total occ. & MIPFs & Chan-Paton Group & Spectrum & \multicolumn{1}{c|}{ x } & Solved \\
\hline 11347 & $32\left(^{* *}\right)$ & 1 & $U(3) \times U(2) \times U(1) \times U(1)$ & VVVV & $1 / 2$ & \\
11462 & $32\left(^{*}\right)$ & 1 & $U(3) \times U(2) \times U(1) \times U(1)$ & VVVV & $1 / 2$ & \\
12327 & 24 & 1 & $U(3) \times U(3) \times U(3)$ & VVV & $1 / 2$ & \\
15824 & 8 & 1 & $U(3) \times U(2) \times U(1) \times U(1)$ & VVVV & 0 & \\
15846 & 8 & 1 & $U(3) \times U(2) \times U(1) \times U(1)$ & VVVV & $1 / 2$ & \\
16674 & 6 & 1 & $U(3) \times U(2) \times U(1)$ & AVT & $1 / 2$ & Y! \\
17055 & 4 & 1 & $U(3) \times U(2) \times U(1) \times U(1)$ & VVVV & $*$ & \\
19345 & 1 & 1 & $U(5) \times U(2) \times O(3)$ & ATV & 0 & \\
\hline \hline
\end{tabular}

The first 25 models are all relatives of the $U(3) \times S p(2) \times U(1) \times U(1)$ models that dominated the search results of [60]. If we classify them according to the pattern and number of intersections, these 25 models come from only three classes. First there are models where both anti-quarks and both anti-leptons come from the same bi-fundamental, like in leftright symmetric models. Secondly, there are models where the family multiplicity of the anti-quarks and anti-leptons are absorbed in Chan-Paton multiplicities of c. Finally, there are models where both happens. Further variations on top of these classes include unifying the baryon and lepton brane to get a Pati-Salam-like structure, and other brane unifications. Models 17 and 18 occur with the same frequency because they are closely related. They only differ by a traceless generator $\operatorname{diag}\left(\frac{1}{3}, \frac{1}{3},-\frac{2}{3}\right)$ from the $U(3)$ factor contributing to $Y$. There are several other cases of closely related models with identical frequencies. In the bottom part of the table we display several lines of special interest, some of which will be discussed in more detail below.

Entry nr. 26 in the table is the first one that cannot be viewed as a relative of the "Madrid model". It has $x=0$ and three anti-symmetric tensors on the QCD and the weak brane. It can be viewed as a broken $S U(5)$ model.

There exist several infinite series of models. In the top of the list one can observe the beginning of the series $U(2 n) \times S p(2) \times G, n>2$, where $G$ can be $O(2), O(6), S p(2)$ or $S p(6)$, with a chiral spectrum consisting of $\frac{6}{N_{c}}(V, 0, V)+3(V, V, 0)$.

In column 2 we indicate between parentheses if a certain type of model was searched for in [60], and how often it was found. It is interesting to compare this with table (5.1). Observe that the number of four-stack configurations we consider in the present paper is considerably smaller than in [60], but nevertheless we recover a large fraction of the standard model configurations of that paper. For example, in [60], $2.8 \times 10^{15}$ configurations of type USUS were examined, in the present paper only $2.6 \times 10^{14}$, ten times less. Nevertheless, we have already found about half of the standard model configurations. This is because the number of brane configurations is dominated by cases with a large number of branes, but very few standard model spectra. This in particular true for the charge conjugation invariant (the simplest case, for which the boundary coefficients were derived by Cardy [40]) which in essentially all cases has by far the largest number of 
boundaries. The explanation may be that a non-trivial MIPF tends to fold over a CalabiYau manifold several times, thus increasing the typical intersection numbers. A prelimary scan of the intersection numbers suggests that the average intersection number for a Cardy invariant does not differ much from the average over all MIPFs. The correlations between intersection numbers as described in section 5.4.4 do however seem to differ significantly. If and how this influences the occurences of standard models is not clear yet.

There are in total three cases with an $S U(3) \times S p(2) \times U(1) \times U(1)$ Chan-Paton group and only bi-fundamentals, namely nr. 30, nr. 343 and $\mathrm{nr}$. 4996. The first two were also searched for in [60], and we find most of them back. They are distinguished by having a massless (nr. 30) or massive (nr. 343) $B-L$ gauge boson. The third one differs in the way quarks and leptons end on branes $\mathbf{c}$ and $\mathbf{d}$. It does not have a lepton number symmetry, and was not considered in [60].

The remaining models considered in [60] have a $U(2)_{\mathbf{b}}$ group instead of $S p(2)_{\mathbf{b}}$. Here a direct comparison is harder, because this splits into many subclasses, which differ in the way the doublets are divided into $(2)$ and $(\overline{2})$ representations of $U(2)$. The cases indicated by a single $(*)$ are models considered in [60] that have a massless $B-L$ boson. In total 131704 such configurations were found in that paper. For three of them we found tadpole solutions; they correspond to the three "type- 1 " models in table 4 of [60]. The ones indicated by $(* *)$ have a massive $B-L$ boson. Only 1306 of these were found in [60], and in no case the tadpole conditions could be solved.

Perhaps the most standard Chan-Paton group for standard model realizations is $U(3) \times$ $U(2) \times U(1) \times U(1)$. The total number of spectra with that CP-group on the complete list is 281. Of these, 19 have a purely bi-fundamental spectrum, and among these 19 there are 17 with $x=\frac{1}{2}$, one with $x=0$ and one with $x=*$. Of the $17 x=\frac{1}{2}$ models, 13 are variations on the "Madrid" model, discussed above. The fourth $x=\frac{1}{2}$ model with a tadpole solution is a model where of the additional $U(1)$ s only $Y$ stays massless, but without a well defined lepton number.

All these 19 purely bi-fundamental models are shown in table (5.6). In addition we show all $U(3) \times U(2) \times U(1) \times U(1)$ configurations that occur more frequently than the first purely bi-fundamental model, nr. 543. These are models with anti-symmetric $U(3)$ tensors. All of them are broken $S U(5)$ models, except nr. 303, which is a broken flipped $S U(5)$ variation of nr. 304.

\subsubsection{Intersection statistics}

Note that only a very small fraction of the allowed bottom-up models is actually realized as top-down configurations. ${ }^{12}$ In this section we try to analyse what decreases the chances of a model to be realized.

A model is realized if we can find a set of stacks for which the intersection numbers coincide with that of the model. In first approximation the Chan-Paton multiplicities do not restrict this, because these numbers become relevant only through the tadpole

\footnotetext{
${ }^{12}$ All results in this section concern brane configurations prior to tadpole cancellation.
} 
conditions. There is one exception: Configurations where the standard model part of the configuration already made it impossible to satisfy the tadpole conditions were discarded.

To make an estimate which models are more likely to occur we therefor study the likelihood of certain patterns of intersections to occur. For a selection of roughly $44 \%$ of the MIPFs we have gathered statistical data of the intersection matrix. For each brane we have calculated the intersection numbers with all possible other branes. In figure 5.1 we plot for all branes of our sample the distribution of chiral bi-fundamentals and chiral tensors. On the horizontal axis is the absolute value of the chirality, and on the vertical axis the total

Figure 5.1: Number of chiral tensors and bi-fundamentals for a selection of branes.

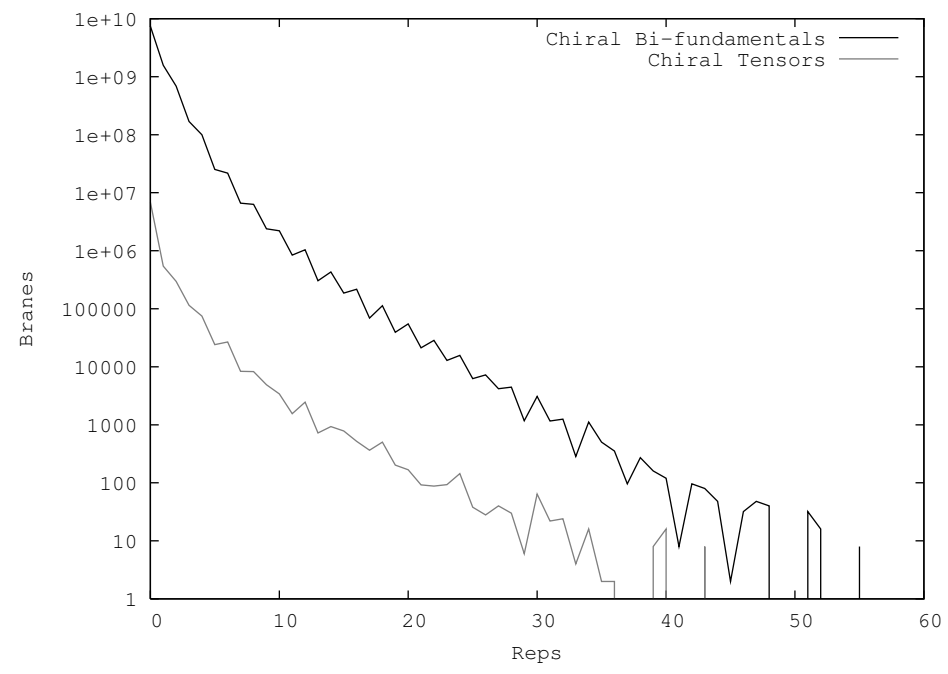

number of occurrences on a log-scale. We have split this in two classes. If this intersection is between a brane and it is conjugate it corresponds to a chiral tensor. If it is between two unrelated branes, it corresponds to a state living in a bi-fundamental representation. Clearly - and not unexpectedly - the number bi-fundamentals is much greater than the number of chiral tensors. This can be intuitively understood by realizing that a brane has a much bigger chance intersecting with any brane yielding a bi-fundamental than intersecting with one specific brane (namely its conjugate), yielding a chiral tensor. An important feature of the distribution that stands out is that higher intersection numbers are exponentially suppressed. An even-odd effect is also visible.

If we assume that the chance that two branes intersect with the same third brane is independent, we can estimate the chance that a certain pattern of branes intersections occurs, by multiplying the relative frequencies of the intersection numbers separately. If the number of branes a certain brane intersects with is large and number of intersection one wants to restrict is small compared to the total number, this is probably a good assumption. One can always remove unwanted intersections with other branes setting the Chan-Paton multiplicity to zero, removing these branes altogether. For each brane we have calculated what percentage of the other branes it intersects with. In figure 5.2 on the next page we 
show the frequency of these percentages. One can see that the majority of the branes

Figure 5.2: Percentage of non-zero intersections per Brane

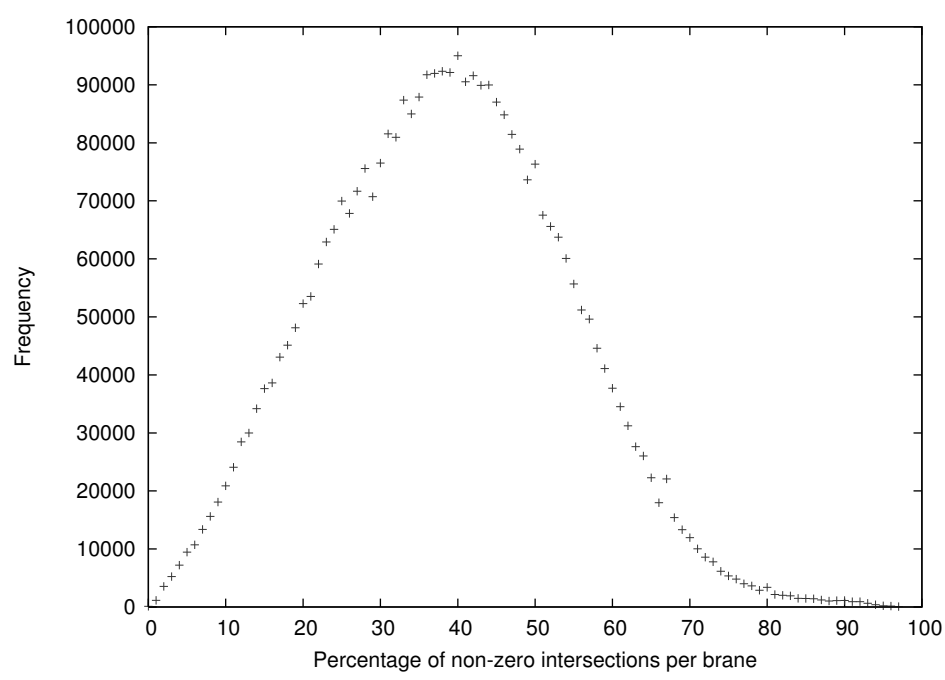

indeed intersect a sizeable fraction of the total number of available branes, with an average of almost 38\%. In our search we have restricted the number of branes contributing to the standard model realizations with up to four branes. The number of available branes is typically in the order of hundreds ( on average it is 545 ) so the second requirement is also satisfied.

There are some intersection numbers that are potentially correlated. The intersection pattern between complex brane $a$ and complex brane $b$ and between brane $a, b^{c}$ is in principle just a pattern of three independent branes. However one can not choose brane $b^{c}$ independently from $b$, the relation is fixed by the orientifold choice. To study how strong these correlations are, we have calculated for complex branes the intersection numbers with all possible conjugate pairs. This is shown in figure 5.3(a) on page 152. On the horizontal and vertical axis are the chirality of intersection $a$ with $b$ and $a$ with $b^{c}$ respectively. These are denoted by $\Delta_{a b}$ and $\Gamma_{a b} \equiv \Delta_{a b^{c}}$ (see section 5.4.6). The color coding depicts the frequency of a certain combination of intersection numbers on a log-scale. For comparison the plot in 5.3(b) on page 152 shows the same set of intersection numbers, but now randomly paired. These figures clearly shows that there is a strong correlation for the intersection numbers to be equal up to a minus sign. Much stronger than what would expect for independent numbers. If one fixes one intersection number to a value unequal to zero, the chance for the intersection number with the conjugate brane being equal is typically of the same order as for it being zero. This despite the fact that intersection number zero is by far the most abundant.

A second source of correlations one has to consider is those in the number of chiral tensors. One can not remove unwanted self intersections like one can do for bi-fundamentals. 
At the moment one picks a complex brane, the number of symmetric and antisymmetric tensors is fixed. In figure 5.4 on the following page we have plot the number of occurrences of combinations of the number of symmetric and number of antisymmetric tensors. On the horizontal axis is the chirality of the antisymmetric tensor, vertical the symmetric tensors. From this figure one can see that the distribution deviates strongly from random pairs of numbers (which would have looked similar to fig. 5.3(b)). The distribution is roughly circular symmetric, which means that the chance of finding a certain combination of symmetric and antisymmetric tensors decreases roughly with the sum of the squares. The distribution is elongated around the axis where the number of symmetric tensors is equal to the number of antisymmetric ones. There is also a slight enhancement for pairs where the number of symmetric tensors is precisely opposite to the number of antisymmetric tensors.

Putting together these consideration we can assign to each model a number which indicates how likely it is to be found:

$$
P=\prod_{a \in \mathcal{R}} P(\mathcal{R}) \prod_{a \in \mathcal{C}} P(\mathcal{C}) \prod_{a \in \mathcal{C}} P\left(A_{a} ; S_{a}\right) \prod_{a \neq b \in \mathcal{C}} P\left(\Delta_{a b} ; \Gamma_{a b}\right) \prod_{a \in \mathcal{C}, b \in \mathcal{R}} P\left(\Delta_{a b}\right)
$$

The numbers $\Delta_{a b}, S_{a}$ and $A_{a}$ together with the complex/real-ness of a brane specify the model. With $P(X)$ we mean the relative frequency of $X$ in its distribution. For example $P\left(A_{a}=3 ; S_{a}=0\right)$ means the number of times a unitary brane has chirality 3 for its antisymmetric tensors and 0 for its symmetric tensors divided by the number of pairs we have calculated. The first two factors are the chances to find a real or complex brane. $70 \%$ the branes are complex. The third factor gives for all complex branes the chances to find the given number of chiral tensors. For all pairs of complex branes we multiply by the chance of finding the combination of $\Delta_{a b}$ and $\Gamma_{a b}$. Finally for all intersections between real and complex branes we multiply with its probability. There is of course no factor for the intersection between two real branes, those are always non-chiral.

We can now try to see if this estimate of the probability makes any sense. In figure 5.5 on page 153 we have plot the value of $\log (P)$ versus the number of realizations for all chiral inequivalent intersection patterns we found. We also plotted per integer value of $\log (P)$ the maximum number of realizations per model and the average number of realizations found per model. It is clear that the calculated probability for a chiral intersection pattern can not be used to make predictions for the number of occurrences of a single model in the search we conducted. The maximal and average number of realizations do seem to have a clear correlation with $\log (P)$. Part of the spread in the number of occurrences for a given value of $\log (P)$ can be understood by the fact that in the search other properties than the chiral intersection numbers did influence the number of realizations we found. The search algorithm was set up to find an embedding of the standard model gauge group in the Chan-Paton group with the correct number of chiral particles, in principle solvable tadpole conditions and a massless hypercharge. Not to just find chiral intersection patterns. For example, a chiral intersection pattern for which the Chan-Paton multiplicity of branes $c$ or $d$ are not restricted have the advantage that there is more freedom to satisfy the hypercharge conditions. 


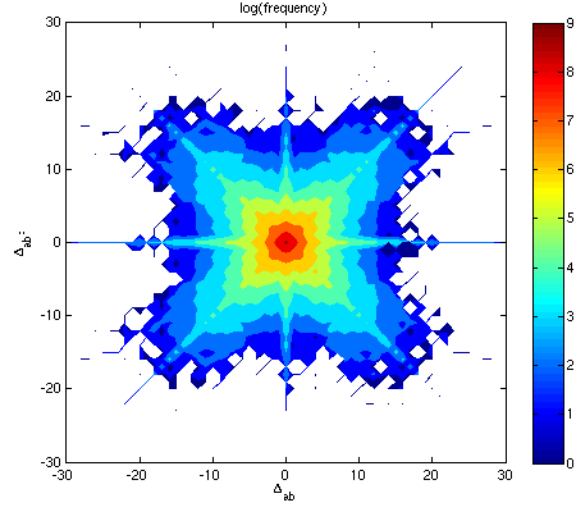

(a) $\Delta_{a b}$ vs $\Gamma_{a b}$

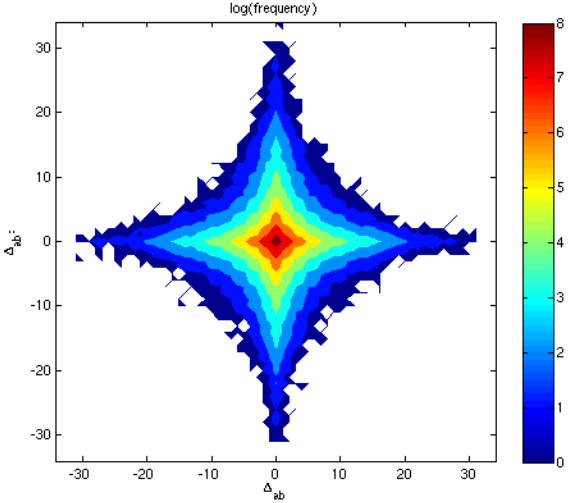

(b) Random pairs

Figure 5.3: Correlations in the intersections of conjugate branes

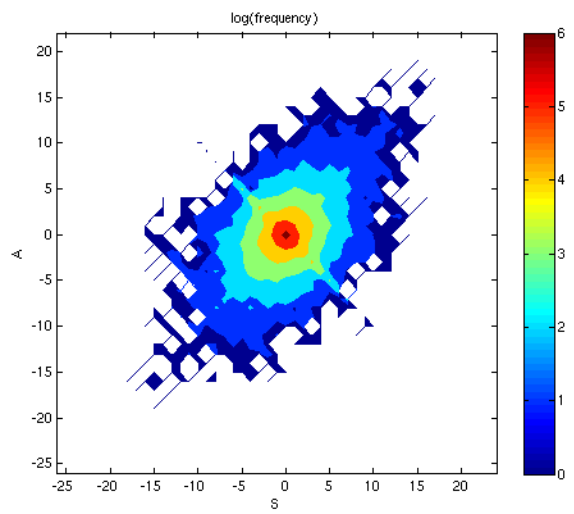

Figure 5.4: Correlations in the number of symmetric and antisymmetric tensors

\subsubsection{Standard model brane configurations not found}

With the measure for probability of an intersection pattern which we defined in the previous chapter we can try to understand why so many bottom-up models are not realized.

Let us first consider the second row from table 5.5 on page 144. These are models with at most three singlet neutrinos, no mirrors and up to one Higgs pair. These restrictions give the possibility of 10680 Bottom-Up models of which only 16 where found. If we calculate the frequency of $\log (P)$ for Bottom-up and Top-Down, we see that most BottomUp models have $-37>\log (p)>-45$. This range and the number of possible Bottom-Up models are bigger then one would maybe expect on the basis of the freedom the restriction to no mirror gives. This can be understood by observing that a lot of bottom-up models use the possibility to cancel anomalies with chiral tensors which are non-chiral with respect to $G_{s m}$. Another source for proliferation of the number of models and the low values for 


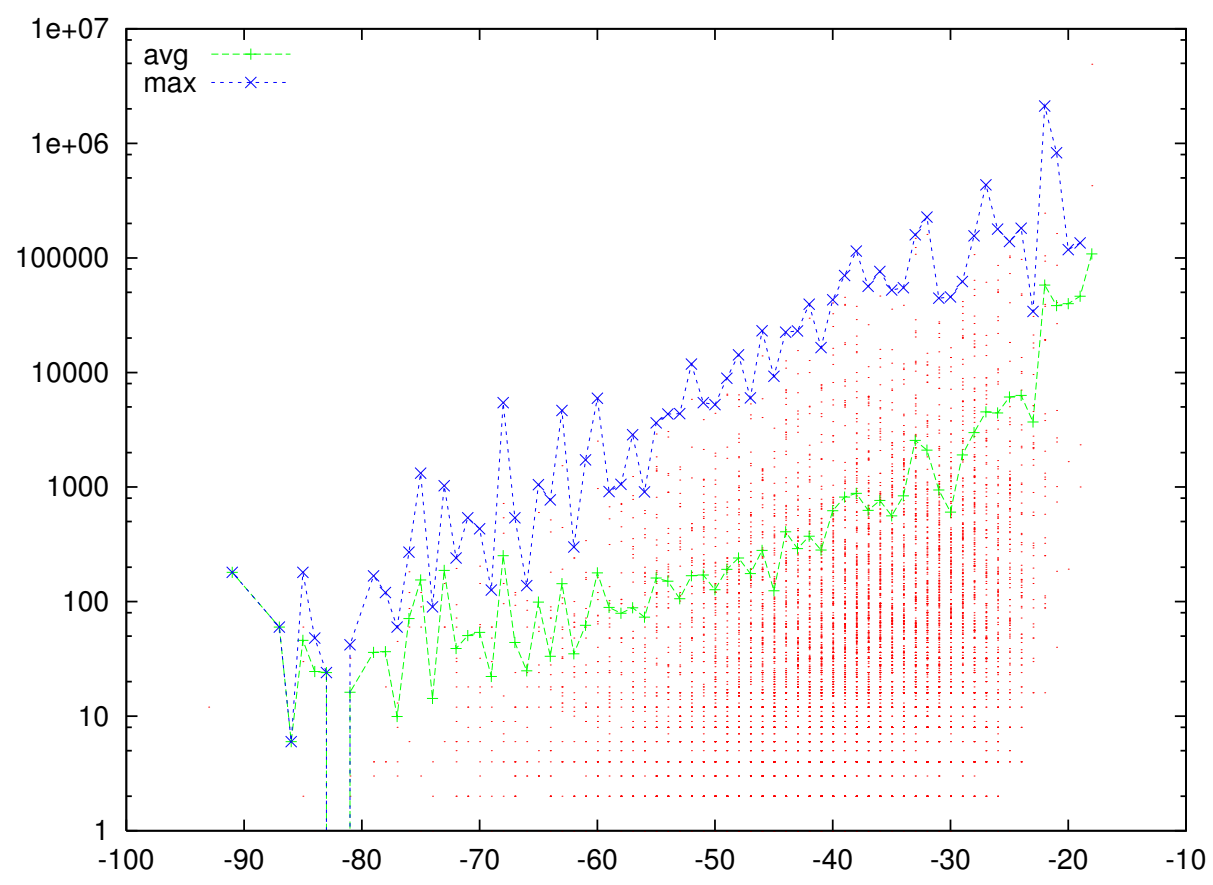

Figure 5.5: $\log (\mathrm{P})$ vs number of realizations for all chiral inequivalent models

$\log (P)$ is distributing family number over several branes.

For the third row from table 5.4 on page 143 (models with up to three mirrors, up to three Higgs and up to six singlet neutrinos) we plotted in figure 5.6 on the following page the values for $\log (P)$ for both the possible Bottom-up and the found Top-Down models. Again it is clear that the majority of the Bottom-Up models have very negative $\log (P)$, with a maximum around -57 . For values of $\log (P)$ greater than -30 a significant number of the possible models where found. For the more negative values some sporadic models where also found, here the possibility of finding a model with a certain value of $\log (P)$ was enhanced by the fact that more models with that value exist. Realizing the fact that the number of Bottom-Up models rises exponentially between $-30>\log (P)>-50$ (and assuming other classes display similar behaviour) implies that the line for the average number of occurrences in 5.5 would have been much steeper if would have averaged over the number of possible models.

If we take the probability interpretation of (5.14) seriously we can use the number of intersection patterns we searched through (see table 5.1) to predict that a pattern with a certain probability will occur $3.610^{15} \times P$ times. For the fixed set of models we are currently focussing on we know how many possible realizations there are for a certain value of $\log (P)$. Using this number to calculate the average number of found models for a certain value $\log (P)$, we can compare with the prediction (see 5.7).

Hypercharge embeddings with $x=-1 / 2,3 / 2$ were not found at all. This is not too surprising given the fact that there are only 22 Bottom-Up models with an average $\log (P)$ 


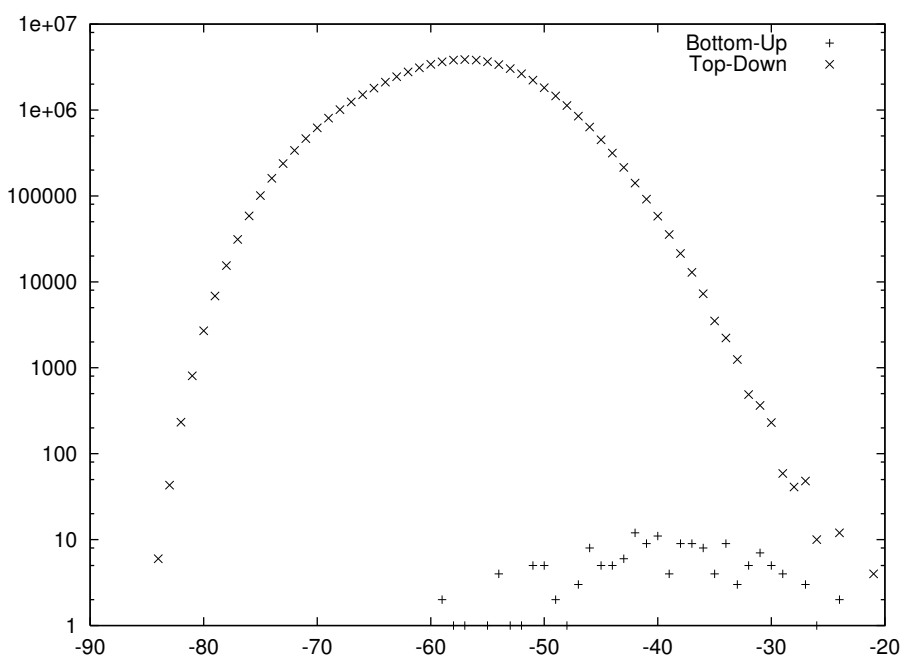

Figure 5.6: $\log (\mathrm{P})$ vs average and maximum Frequency

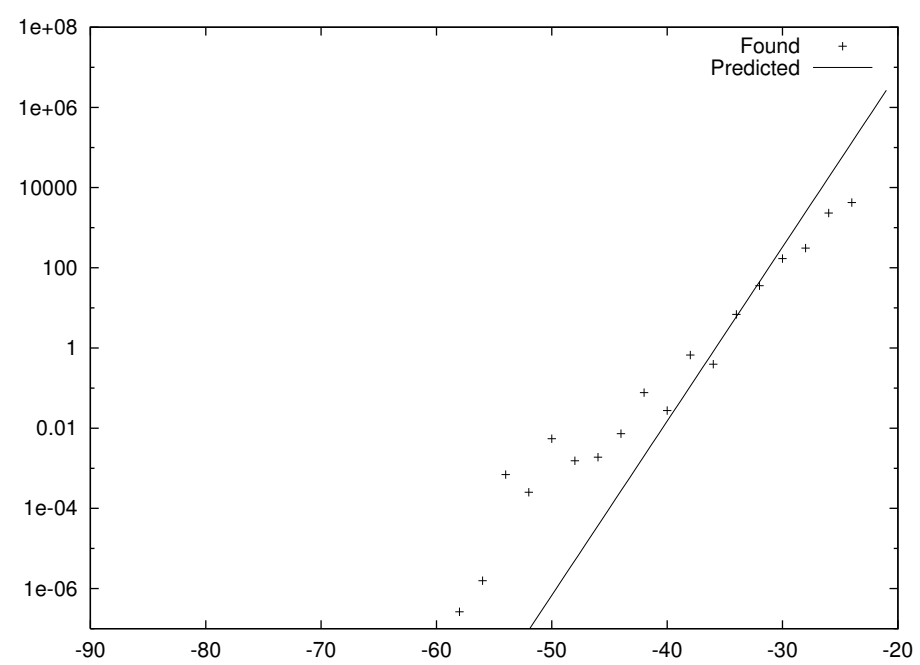

Figure 5.7: Predicted and average number of found models with $\nu^{c} \leq 6, H \leq 3, M \leq 3$.

of -33 . The full list of 19345 configurations does contain some genuine $x=1$ models, with $x$ fixed to that value by the quark and lepton charges. There is a total of 17 distinct ones (for none of these we found a solution to the tadpole conditions). Only one of these, nr. 2919, has an orthogonal group on the $\mathbf{d}$-stack, but it is not identical to one of the simple models written down in section 5.3.5. It has a Chan-Paton group $U(3) \times U(2) \times U(2) \times O(3)$, with both a $\mathrm{C}$ and a D brane on stack c. This model was found a total of 1024 times for just two MIPFs. The purely unitary $x=1$ models 1024 and 2624 occur more frequently. Another noteworthy absence in this class is the type B,B' model introduced in $[12,11]$. 
These models have a Chan-Paton group $U(3) \times U(2) \times U(1) \times U(1)$, and the type-B model only has bifundamentals, whereas type-B' has anti-symmetric tensor on $U(2)_{\mathbf{b}}$. However, all $x=1$ models we found have a $U(2)$ group on brane $\mathbf{c}$, and all have anti-symmetric tensors both on branes $\mathbf{b}$ and $\mathbf{c}$. Some of these are similar to the models of $[12,11]$, but not identical. Note that the type B,B' models of $[12,11]$, in order to be free of cubic anomalies in the two $U(1)$ factors and the $U(2)$, need $U(2)_{\mathbf{b}}$-chiral Higgs pairs and antisymmetric $U(1)$ tensors, as discussed in section 5.3.5. This suppresses their statistical likelihood.

Another model proposed in the literature that did not emerge in our search is model $\mathrm{C}$ of [9]. This is a $U(3) \times U(2) \times U(1)$ model with three $G_{\mathrm{CP}}$-chiral neutrinos appearing as anti-symmetric tensors of $U(2)$. However, model $\mathrm{nr} 7464$ in table (5.6) is similar to it. It has exactly the same structure as model $\mathrm{C}$ of [9], after replacing $U(2)$ by $S p(2)$. Then such neutrinos necessarily become non-chiral. Also both anti-quarks (and anti-leptons) now come from the intersection of a real and a complex brane instead of two complex ones, increasing the chances for this model. If we use formula (5.14) to make this more concrete we find that model $\mathrm{C}$ acquires an additional factor of $10^{-5}$ for its probability. Model $\mathrm{nr}$. 7464 occurred only 108 times (and without tadpole solutions). Its presence suggests that there is no fundamental obstacle to finding model C, but that it is simply statistically disfavored.

On the full list of 19345 models there are 150 of the class $x=*$. All of them are truly orientable, i.e the possibility of having anti-symmetric $U(1)$ tensors that do not contribute massless states does not occur. Only one has Chan-Paton group $U(3) \times U(2) \times U(1) \times U(1)$. It is indeed precisely the model (5.8) shown in section 5.3. This simple model occurs only four times (nr. 17055), and just for one MIPF (and without any tadpole solution to tadpole cancellation). A statistical disadvantageous feature of this model is the fact that it needs a chiral intersection number $\Delta_{a b}=6, \Gamma_{a b}=0$, which are quite rare. More importantly the fact that it is orientable means that for each representation it needs an independent intersection; All six intersection numbers are non-zero. Not surprisingly there are other unoriented $U(3) \times U(2) \times U(1) \times U(1)$ configurations with only bi-fundamentals that do occur much more frequently, as discussed above. For example nr. 543 in table (5.6) occurs 20176 times. This is a standard "Madrid"-type configuration. It has only four non-zero intersections and has using formula (5.14) a factor $10^{5}$ higher probability.

One can also make an interesting observation regarding the occurrence of chiral tensors in comparison to non-chiral ones. In fig. (5.8) we list for all branes in all 33012 non-zero tension orientifolds the distribution of chiral and non-chiral tensors (separately for adjoints and the other rank-2 tensors). Note that this includes all branes in all Gepner orientifolds with non-zero-tension O-planes, not just those considered in the present paper. Clearly the chiral distribution falls off much faster than the non-chiral ones.

\subsubsection{Higgs, neutrino and mirror distributions}

Figures (5.10(a)), (5.10(b)) and (5.9) and show the distribution in terms of the number of Higgs, right-handed neutrinos and mirror pairs. On the vertical axis we show the 
Figure 5.8: Number of chiral and non-chiral tensors for all single branes.

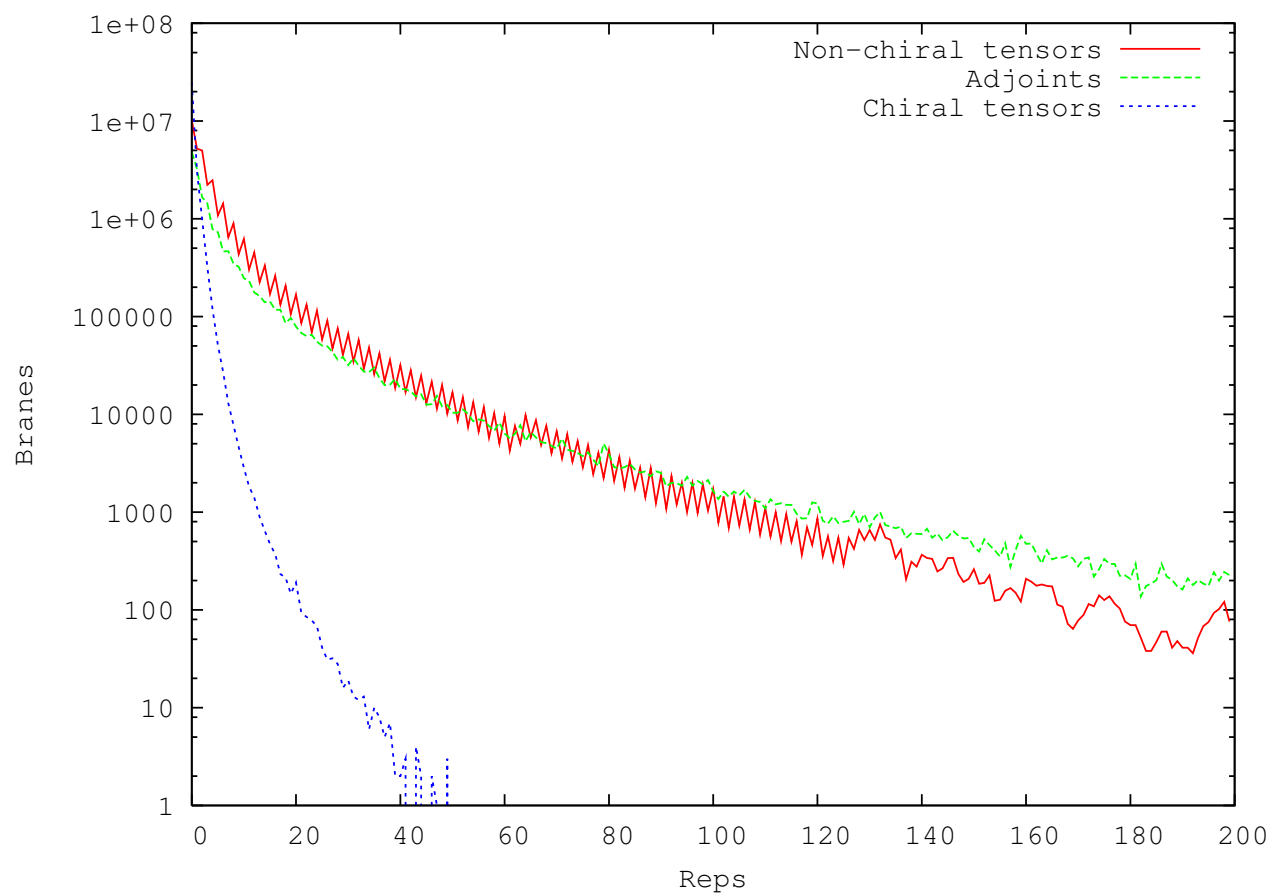

total number of three and four-brane configurations that have a chiral standard model spectrum, plus the number of Higgses/neutrinos/mirrors indicated on the horizontal axis. Just as all data in this section, these numbers refer to brane configurations prior to tadpole cancellation. The Higges/neutrinos/mirrors are $G_{\mathrm{CP}}$ chiral but of course $G_{\mathrm{SM}}$ non-chiral. In addition to these particles, the massless spectrum may contain $G_{\mathrm{CP}}$-non-chiral particles with the same standard model transformation properties. Since we classify models modulo full non-chiral matter, we have no general information about such particles. The mirror count is the total of all mirror pairs of quark and charged lepton weak singlets, as well as quark doublets (in this case mirrors can occur only for $x=\frac{1}{2}$ ). The Higgs count refers to $\left(1,2, \frac{1}{2}\right)+\left(1,2,-\frac{1}{2}\right)$ pairs; for example the MSSM has one such pair. Note that these pairs could also be viewed as lepton doublet mirror pairs. The distinction can be made in models with a well-defined lepton number, but since we are not insisting on that we simply count all such pairs as candidate Higgs. Once one (or more) of these candidates acquires a v.e.v, one may discuss if lepton number violation is absent or acceptably small.

Finally fig. (5.10(b)) shows the distribution of the total number of standard model singlets in the $G_{\mathrm{CP}}$-chiral spectrum.

In all three plots two lines are visible. The top line corresponds to multiplicities that are $0 \bmod 3$, and the lower to multiplicities that are not $0 \bmod 3$. The former occur more frequently due to anomaly cancellation and the fact that we require the presence of three chiral families. In some classes of models this imposes a mod 3 constraint on the 


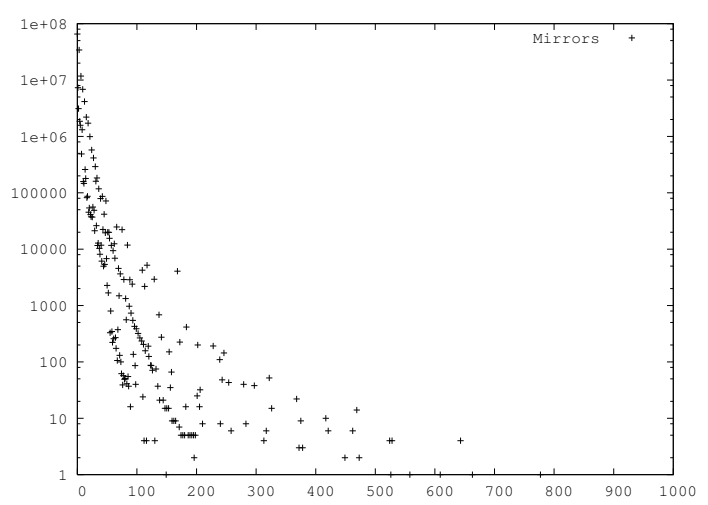

Figure 5.9: Mirror distribution

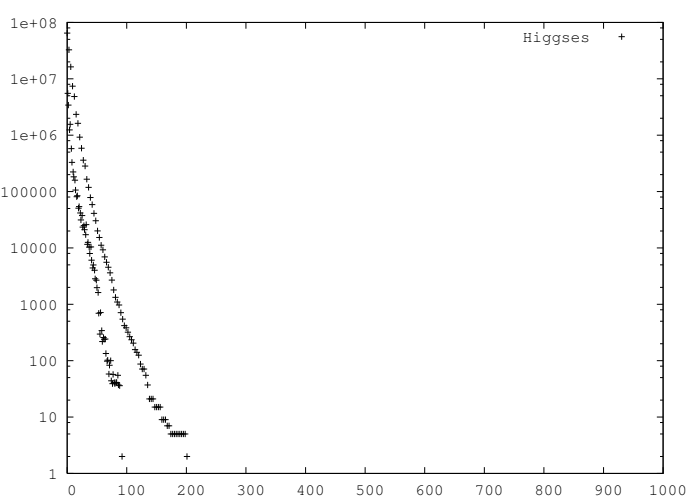

(a) Higgs pairs

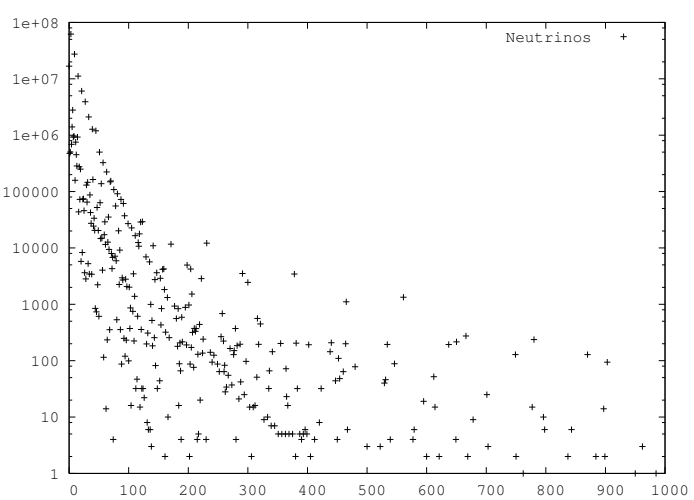

(b) Right-handed neutrino

Figure 5.10: Distributions for all standard model configurations.

multiplicities of Higgses, mirror or neutrinos. This feature is clearest in the Higgs plot, because the Higgs is in a definite, and non-trivial standard model representation with few $G_{C P}$ realizations. It is less clear in the neutrino plot, because there are often many ways of making neutrinos. The models with huge numbers of (right-handed) neutrino candidates usually contain a large factor $G_{\mathbf{c}}$ or $G_{\mathbf{d}}$, with neutrinos coming from rank-2 tensors.

\section{Appendix: The unbiased search algorithm}

We introduce the following notation (in the following $a, b, \ldots$ are generic boundary state labels, not to be confused with the specific labels $\mathbf{a}, \mathbf{b}$ for the QCD, weak and other standard model branes)

$$
N_{a b}=\sum_{i} A_{a b}^{i} \chi_{i}(m=0, L)
$$


where $A_{a b}^{i}$ are the unoriented annulus coefficients and $\chi_{i}(m=0, L)$ is the character of representation $i$, restricted to massless, left-handed fermions. The boundary conjugates of $a$ and $b$ are denoted by $a^{c}$ and $b^{c}$. If we consider two complex boundaries $a$ and $b$, there is $a$ total of four quantities relevant for the massless spectrum, namely $N_{a b}, N_{a b^{c}}, N_{a^{c} b}$ and $N_{a^{c} b^{c}}$. The chiral information is contained in two quantities, namely

$$
\Gamma_{a b}=N_{a b}-N_{a^{c} b^{c}}
$$

and

$$
\Delta_{a b}=N_{a b^{c}}-N_{a^{c} b}
$$

If either $a$ or $b$ are real, we set $\Delta=0$. If both $a$ and $b$ are real $\Gamma=\Delta=0$. Furthermore we define the chiral numbers of anti-symmetric and symmetric tensors

$$
A_{a}=\frac{1}{2}\left(N_{a a}-N_{a a^{c}}-M_{a}+M_{a^{c}}\right)
$$

and

$$
S_{a}=\frac{1}{2}\left(N_{a a}-N_{a a^{c}}+M_{a}-M_{a^{c}}\right)
$$

where $M$ is the Moebius contribution

$$
M_{a}=\sum_{i} M_{a}^{i} \chi_{i}(m=0, L)
$$

Our search procedure is as follows

1. Consider all orientifold choices that have non-zero tension. We will label them by an integer $\ell$. This sequential label corresponds to some choice of the discrete parameters of the RCFT, called "Klein bottle currents" and "crosscap signs" [69]. The sign of the tension of the corresponding O-plane is a free parameter in RCFT constructions, and we choose it negative. We denote its value as $T_{O}^{\ell}$.

2. For each $\ell$, consider all candidates for brane a subject to the conditions

(a) Brane a is complex.

(b) The brane tension $T_{\mathbf{a}}$ satisfies $6 T_{\mathbf{a}}+T_{O}^{\ell}<0$, because the complete configuration must satisfy the dilaton tadpole condition $\sum_{x} T_{x}+T_{O}^{\ell}=0$, and all $T_{x}$ are positive. This is needed in order to accommodate further branes.

(c) There are no chiral symmetric tensors.

3. For each $\ell$ and $\mathbf{a}$, consider all candidates for brane $\mathbf{b}$ that satisfy the following conditions

(a) The CP group associated with $\mathbf{b}$ is not orthogonal.

(b) The brane tension $T_{\mathbf{b}}$ satisfies $6 T_{\mathbf{a}}+2 T_{\mathbf{b}}+T_{O}^{\ell}<0$, if $\mathbf{b}$ is real, $6 T_{\mathbf{a}}+4 T_{\mathbf{b}}+T_{O}^{\ell}<0$ if $\mathbf{b}$ is complex. 
Appendix: The unbiased search algorithm

(c) There are three chiral bi-fundamentals $(3,2)$. These are only counted chirally, i.e additional mirror pairs are allowed. If brane $\mathbf{b}$ is complex, the chiral total of $(3,2)$ and $\left(3,2^{*}\right)$ must be three.

(d) There are no chiral symmetric tensors. This is the an application of the condition mentioned in section 5.2, that $R_{C P}^{\text {chir }}$ should not yield anything more exotic than mirrors. It is not absolutely essential here, but it gives a useful early limitation on the number of solutions.

4. For each $\ell, \mathbf{a}$ and $\mathbf{b}$ consider all candidates $\mathbf{c}$ that satisfy:

(a) Brane $\mathbf{c}$ is allowed at least once by the dilaton tension constraint.

(b) We need weak singlet anti-quarks. They can come from anti-symmetric tensors of brane $\mathbf{a}$ or from bi-fundamentals between brane $\mathbf{a}$ and either branes $\mathbf{c}$ or $\mathbf{d}$. Since the anti-symmetric tensors can have only one charge, at least three antiquarks must come from bi-fundamentals. There is no a priori ordering between branes $\mathbf{c}$ and $\mathbf{d}$. To prevent double-counting, we will impose the condition that brane $\mathbf{c}$ must provide more anti-quarks plus mirrors than brane $\mathbf{d}$. More precisely, we will impose the condition $N_{\mathbf{c}}\left(\left|\Gamma_{\mathbf{a c}}\right|+\left|\Delta_{\mathbf{a c}}\right|\right) \geq N_{\mathbf{d}}\left(\left|\Gamma_{\mathbf{a d}}\right|+\left|\Delta_{\mathbf{a d}}\right|\right)$. This ordering condition can only be imposed once we have determined branes $\mathbf{c}$ and $\mathbf{d}$ as well as their CP multiplicities $N_{\mathbf{c}}$ and $N_{\mathbf{d}}$, but at this stage it already implies that $\left(\left|\Gamma_{\mathbf{a c}}\right|+\left|\Delta_{\mathbf{a c}}\right|\right)>0$.

5. Given $\ell \mathbf{a}, \mathbf{b}$ and $\mathbf{c}$ there may be a value for $N_{\mathbf{c}}$ (the CP multiplicity of brane $\mathbf{c}$ ) and a hypercharge choice so that the standard model is already obtained for just three stacks. However, in general a fourth stack is needed (even if there is a valid three-stack solution we will continue looking for a fourth one). Hence we consider all labels $\mathbf{d}$ that satisfy:

(a) At least one of the stacks $\mathbf{b}, \mathbf{c}$ and $\mathbf{d}$ is complex. Otherwise it would be impossible to obtain chiral leptons.

(b) At least one of the quantities $S_{\mathbf{d}}, A_{\mathbf{d}}, \Gamma_{\mathbf{a d}}, \Gamma_{\mathbf{b d}}, \Gamma_{\mathbf{c d}}, \Delta_{\mathbf{a d}}, \Delta_{\mathbf{b d}}$, and $\Delta_{\mathbf{c d}}$ is non-zero. Otherwise brane $\mathbf{d}$ can be regarded as part of the hidden sector.

6. Now we have collected an orientifold and four branes $\mathbf{a}, \mathbf{b}, \mathbf{c}, \mathbf{d}$ and we have to determine the CP multiplicities of the last two branes. Because, by assumption, any further branes are in the hidden sector and cannot contribute chiral states to the four CP groups, all cubic anomalies must now cancel. This gives at least two and at most four equations for the two quantities $N_{\mathbf{c}}$ and $N_{\mathbf{d}}$. The following things can happen:

(a) There are two independent equations that fix $N_{\mathbf{c}}$ and $N_{\mathbf{d}}$. Both are positive integers, and are even for symplectic groups. Now we can move on to the next stage, and compute the Y-charge combination (see below). 
(b) The equations are inconsistent, do not have positive integer solutions, or have a solution with an odd CP multiplicity for a symplectic group. In all these cases the configuration $(\ell, \mathbf{a}, \mathbf{b}, \mathbf{c}, \mathbf{d})$ must be rejected.

(c) There is only one independent equation. This means that only a linear combination $f_{\mathbf{c}} N_{\mathbf{c}}+f_{\mathbf{d}} N_{\mathbf{d}}$ is fixed. If this happens we consider all values of $N_{\mathbf{d}}$ or $N_{\mathbf{c}}$ (if $f_{\mathbf{c}}=0$ ) between 1 and the maximum allowed by the dilaton tadpoles, and attempt the next stage (computing $Y$ ) for all of them.

(d) There is no equation at all. This means that all anomalies cancel independent of $N_{\mathbf{c}}$ and $N_{\mathbf{d}}$. This can only happen if $A_{\mathbf{a}}=6$. If $A_{\mathbf{a}} \neq 6$, there must be chiral bi-fundamentals giving rise to anti-quarks, and their contribution to the $S U(3)$ anomaly depends on $N_{\mathbf{c}}$ or $N_{\mathbf{d}}$, or both. In this case we consider all allowed values of $N_{\mathbf{c}}$ as well as $N_{\mathbf{d}}$ and attempt to determine $Y$.

7. The next step is to compute the standard model $Y$-charge. In general it is a linear combination of the form $Y=\sum_{\alpha} t_{\alpha} Q_{\alpha}+W_{\mathbf{c}}+W_{\mathbf{d}}$, where $Q_{\alpha}$ is the $U(1)$ charge of one of the unitary brane stacks, with $\alpha=(\mathbf{a}, \mathbf{b}, \mathbf{c}, \mathbf{d})$. Real stacks have $Q_{\alpha}=0$. The last two terms are simple Lie-algebra generators in the CP-factors of branes c and $\mathbf{d}$, in other words generators of $S U(N), O(N)$ or $S p(N)$. They can be brought to diagonal form and may therefore be parametrized as traceless diagonal matrices, which in the case of $O(N)$ and $S p(N)$ must have equal numbers of eigenvalues of opposite sign. We first determine the coefficients $t_{\alpha}$. We do this by solving one of the following sets of equations:

- Trace Equations: These are obtained by taking the trace for each of the $S U(3) \times$ $S U(2)$ representations $(3,2),\left(3^{*}, 1\right),(2,1)$ and $(1,1)$. On the phenomenological side, any non-chiral mirror pairs do not contribute to these traces, and on the string theory side $W_{\mathbf{c}}$ and $W_{\mathbf{d}}$ do not contribute. Therefore this gives four equations for at most four variables $t_{\alpha}$.

- Axion Equations: Require absence of axion-Y bilinear couplings. This gives a condition for every axion, and yields in general far more conditions than there are variables. Note that $W_{\mathbf{c}}$ and $W_{\mathbf{d}}$ do not couple to any axions. Since we want rational solutions $t_{\alpha}$ and since the axion couplings are real numbers, the solutions have to be converted to rational numbers of the form $p / q$. We perform that conversion assuming $|q| \leq 1024$.

- Exact Charge Equations: Write down equations for the actual charges (rather than the traces) for each non-zero coefficient $A, S, \Gamma$ or $\Delta$. We write these equations for the maximal eigenvalue in the $\mathbf{c}$ and $\mathbf{d}$ sectors, i.e. for the maximal eigenvalue of $x_{\mathbf{c}}=t_{c} Q_{\mathbf{c}}+W_{\mathbf{c}}$ or $x_{\mathbf{d}}=t_{d} Q_{\mathbf{d}}+W_{\mathbf{d}}$. The right hand side of such an equation must be a valid (mirror) quark or lepton charge, and is determined up to at most an integer $0, \pm 1$. These linear equations can be solved, and limit $x_{\mathbf{c}}$ and $x_{\mathbf{d}}$ to a definite range of integers and half-integers. To determine $t_{\mathbf{c}}$ (and analogously $t_{\mathbf{d}}$ ) we consider all possible multiplicities of the eigenvalue $W_{\mathbf{c}}^{\max }$, 
Appendix: The unbiased search algorithm

between 1 and $N_{\mathbf{c}}$. Given this multiplicity, and the fact that $W_{\mathbf{c}}$ is traceless, we can determine $t_{\mathbf{c}}$. Taking into account all these possibilities (the integer ambiguities and the number of maximal eigenvalues) then gives a set of possible variables for $t_{c}$ and $t_{d}$.

These methods are used successively as needed. The first is the simplest and usually sufficient, and only in rare cases the third method is needed. The $Y$-mass constraint is in any case checked as a condition, if it was not used as equation. Note that the exact charge equations cannot fix all $t_{\alpha}$ if the brane configuration is orientable. In that case these equations have a one-dimensional kernel, and only the axion equations might fully determine $t_{\alpha}$. To summarize, we have following possibilities:

(a) The trace equations completely fix all $t_{\alpha}$. In that case the axion- $Y$ bilinear couplings are computed for this particular $Y$. If they all vanish, we move on to the next step.

(b) The equations do not fix all $t_{\alpha}$. In that case we combine the trace equations with the axion equations.

(c) The trace and axion equations still do not all fix $t_{\alpha}$. In that case we use the exact charge equations to determine the missing coefficient(s) up to a finite set of rational numbers.

(d) The trace plus axion equations do not determine all $t_{\alpha}$ completely, and neither do the exact charge equations. In this case both sets of equations have a nontrivial kernel and there are two possibilities:

i. The kernel vector of the exact charge equations is in the kernel of the trace and axion equations. This means that we can add a set of coefficients $x_{\alpha}$ to $t_{\alpha}$ without affecting the quark and lepton charges, nor the axion couplings. This is a genuine ambiguity, which cannot be resolved by any conditions at our disposal. We fix this ambiguity by setting one of the missing coefficients to a chosen "canonical" value $\left(\frac{1}{6}, 0,-\frac{1}{2},-\frac{1}{2}\right.$ for $t_{a}, \ldots t_{c}$ respectively).

ii. The kernel vector of the exact charge equations is not in the kernel of the trace and axion equations. In this case the equations can be solved by combining them. There is a minor complication due to the fact that the exact charge equations have a range of rational numbers as their right hand side. To deal with this we consider a set of rational values $p / q$ for the missing $t_{\alpha}$. For $q$ we use the smallest common multiple of $24, N_{\mathbf{c}}$ and $N_{\mathbf{d}}$, and we allow all values for $p$ so that $-1 \leq p / q \leq 1$. Since the kernels of the two set of equations do not overlap, there will be at most a few solutions.

All possibilities described above do actually occur.

8. Determining $W_{\mathbf{c}}$ and $W_{\mathbf{d}}$, given $t_{\alpha}$. This is now easy, because the eigenvalues of these generators must lower or raise the value of $Y$ to an allowed quark or lepton charge. Hence at most two distinct eigenvalues are allowed. Since the generators 
must be traceless, this fixes them completely. If the $\mathbf{c}$ or $\mathbf{d}$ groups are orthogonal or symplectic, the two eigenvalues must be equal in number and opposite. Note that for $S U(3) \times S U(2)$ singlets we allow three charges, $0, \pm 1$, but if there is an equal number of charges +1 and -1 this just adds non-chiral pairs. This is a degeneracy, that can be fixed by setting all paired charges to 0 . Hence also in this case at most two distinct $W_{\mathbf{c}}$ or $W_{\mathbf{d}}$ eigenvalues are needed.

9. Finally we count the quarks and leptons, to check that the correct particle multiplicities are obtained.

There is some potential over-counting in the procedure, due to the following reasons

1. If the $\mathbf{b}$-brane is complex, one can interchange $\mathbf{b}$ and $\mathbf{b}^{c}$

2. The choice of $\mathbf{c}$ and $\mathbf{d}$ is interchangeable.

3. The choice of $\mathbf{c}$ and $\mathbf{c}^{c}$ is interchangeable.

4. The choice of $\mathbf{d}$ and $\mathbf{d}^{c}$ is interchangeable.

These degeneracies are fixed as follows. The first one can be dealt with by requiring that there are more chiral representations $(3,2)$ than $\left(3,2^{*}\right)$. Since their total must be three, they cannot be equal. The second one can be fixed by requiring that brane $\mathbf{c}$ produce a larger total number of anti-quarks than brane $\mathbf{d}$, i.e. $N_{\mathbf{c}}\left(\left|\Gamma_{\mathbf{a c}}\right|+\left|\Delta_{\mathbf{a c}}\right|\right) \geq N_{\mathbf{d}}\left(\left|\Gamma_{\mathbf{a d}}\right|+\left|\Delta_{\mathbf{a d}}\right|\right)$. If that still yields equality, we require that brane $\mathbf{c}$ produce more chiral anti-quarks than brane $\mathbf{d}$. A few further constraints of this type may be used to fix the ambiguity completely. To fix the conjugation ambiguities of the $\mathbf{c}$ and $\mathbf{d}$ branes we require that certain chiral quantities associated with these branes are positive. 


\section{Bibliography}

[1] Y. Achiman and B. Stech. In D. E. C. Fries and J. Wess, editors, New Phenomena in Lepton-Hadron Physics, page 303. Plenum, New York, 1979.

[2] G. Aldazabal, E. C. Andres, and J. E. Juknevich. Particle models from orientifolds at gepner-orbifold points. JHEP, 05:054, 2004.

[3] G. Aldazabal, S. Franco, Luis E. Ibanez, R. Rabadan, and A. M. Uranga. D = 4 chiral string compactifications from intersecting branes. J. Math. Phys., 42:3103-3126, 2001.

[4] G. Aldazabal, Luis E. Ibanez, F. Quevedo, and A. M. Uranga. D-branes at singularities: A bottom-up approach to the string embedding of the standard model. JHEP, 08:002, 2000.

[5] Gerardo Aldazabal, Eduardo C. Andres, Mauricio Leston, and Carmen Nunez. Type iib orientifolds on gepner points. JHEP, 09:067, 2003.

[6] C. Angelantonj, M. Bianchi, G. Pradisi, A. Sagnotti, and Ya. S. Stanev. Chiral asymmetry in four-dimensional open- string vacua. Phys. Lett., B385:96-102, 1996.

[7] Carlo Angelantonj, Massimo Bianchi, Gianfranco Pradisi, Augusto Sagnotti, and Yassen S. Stanev. Comments on gepner models and type i vacua in string theory. Phys. Lett., B387:743-749, 1996.

[8] Carlo Angelantonj and Augusto Sagnotti. Open strings. Phys. Rept., 371:1-150, 2002.

[9] I. Antoniadis and S. Dimopoulos. Splitting supersymmetry in string theory. Nucl. Phys., B715:120-140, 2005.

[10] Ignatios Antoniadis, E. Dudas, and A. Sagnotti. Brane supersymmetry breaking. Phys. Lett., B464:38-45, 1999.

[11] Ignatios Antoniadis, E. Kiritsis, and T. Tomaras. D-brane standard model. Fortsch. Phys., 49:573-580, 2001.

[12] Ignatios Antoniadis, E. Kiritsis, and T. N. Tomaras. A d-brane alternative to unification. Phys. Lett., B486:186-193, 2000.

[13] Ignatios Antoniadis, Elias Kiritsis, and John Rizos. Anomalous u(1)s in type i superstring vacua. Nucl. Phys., B637:92-118, 2002.

[14] Gregory G. Athanasiu, Joseph J. Atick, Michael Dine, and Willy Fischler. Remarks on wilson lines, modular invariance and possible string relics in calabi-yau compactifications. Phys. Lett., B214:55, 1988.

[15] Constantin Bachas, Nicolas Couchoud, and Paul Windey. Orientifolds of the 3-sphere. JHEP, 12:003, 2001.

[16] D. Bailin, G. V. Kraniotis, and A. Love. Standard-like models from intersecting d4-branes. Phys. Lett., B530:202-209, 2002. 
[17] David Bailin, Alex Love, and Steven Thomas. A three generation orbifold compactified superstring model with realistic gauge group. Phys. Lett., B194:385, 1987.

[18] P. Bantay. Permutation orbifolds. Nucl. Phys., B633:365-378, 2002.

[19] Roger E. Behrend, Paul A. Pearce, Valentina B. Petkova, and Jean-Bernard Zuber. On the classification of bulk and boundary conformal field theories. Phys. Lett., B444:163-166, 1998 .

[20] Roger E. Behrend, Paul A. Pearce, Valentina B. Petkova, and Jean-Bernard Zuber. Boundary conditions in rational conformal field theories. Nucl. Phys., B570:525-589, 2000.

[21] David Berenstein, Vishnu Jejjala, and Robert G. Leigh. The standard model on a d-brane. Phys. Rev. Lett., 88:071602, 2002.

[22] Oren Bergman, Eric G. Gimon, and Shigeki Sugimoto. Orientifolds, rr torsion, and k-theory. JHEP, 05:047, 2001.

[23] M. Bianchi, G. Pradisi, and A. Sagnotti. Toroidal compactification and symmetry breaking in open string theories. Nucl. Phys., B376:365-386, 1992.

[24] Massimo Bianchi and Jose F. Morales. Anomalies and tadpoles. JHEP, 03:030, 2000.

[25] Massimo Bianchi and Augusto Sagnotti. On the systematics of open string theories. Phys. Lett., B247:517-524, 1990.

[26] Lothar Birke, Jurgen Fuchs, and Christoph Schweigert. Symmetry breaking boundary conditions and wzw orbifolds. Adv. Theor. Math. Phys., 3:671-726, 1999.

[27] Ralph Blumenhagen. Supersymmetric orientifolds of gepner models. JHEP, 11:055, 2003.

[28] Ralph Blumenhagen, Volker Braun, Boris Körs, and Dieter Lüst. Orientifolds of k3 and calabi-yau manifolds with intersecting d-branes. JHEP, 07:026, 2002.

[29] Ralph Blumenhagen, Mirjam Cvetic, Paul Langacker, and Gary Shiu. Toward realistic intersecting d-brane models. Ann. Rev. Nucl. Part. Sci., 55:71-139, 2005.

[30] Ralph Blumenhagen, Lars Görlich, Boris Körs, and Dieter Lüst. Noncommutative compactifications of type i strings on tori with magnetic background flux. JHEP, 10:006, 2000.

[31] Ralph Blumenhagen, Lars Görlich, and Tassilo Ott. Supersymmetric intersecting branes on the type iia $\mathrm{t}^{* *} 6 / \mathrm{z}(4)$ orientifold. JHEP, 01:021, 2003.

[32] Ralph Blumenhagen, Boris Körs, Dieter Lüst, and Tassilo Ott. The standard model from stable intersecting brane world orbifolds. Nucl. Phys., B616:3-33, 2001.

[33] Ralph Blumenhagen, Dieter Lüst, and Stephan Stieberger. Gauge unification in supersymmetric intersecting brane worlds. JHEP, 07:036, 2003.

[34] Ralph Blumenhagen and Timo Weigand. Chiral supersymmetric gepner model orientifolds. 2004.

[35] Ralph Blumenhagen and Andreas Wisskirchen. Spectra of $4 \mathrm{~d}, \mathrm{n}=1$ type i string vacua on non-toroidal cy threefolds. Phys. Lett., B438:52-60, 1998.

[36] L. Borisov, M. B. Halpern, and C. Schweigert. Systematic approach to cyclic orbifolds. Int. J. Mod. Phys., A13:125-168, 1998.

[37] Ilka Brunner. On orientifolds of wzw models and their relation to geometry. JHEP, 01:007, 2002 . 
[38] Ilka Brunner, Kentaro Hori, Kazuo Hosomichi, and Johannes Walcher. Orientifolds of gepner models. 2004.

[39] Andrea Cappelli and Giuseppe D'Appollonio. Boundary states of $\mathrm{c}=1$ and $3 / 2$ rational conformal field theories. JHEP, 02:039, 2002.

[40] John L. Cardy. Boundary conditions, fusion rules and the verlinde formula. Nucl. Phys., B324:581, 1989.

[41] J. A. Casas and C. Munoz. Three generation $\mathrm{su}(3) \mathrm{xu} \operatorname{su}(2) \mathrm{x} \mathrm{u}(1)$-y models from orbifolds. Phys. Lett., B214:63, 1988.

[42] J. A. Casas and C. Munoz. Three generation su(3) x su(2) x u(1)-y x u(1) orbifold models through fayet-iliopoulos terms. Phys. Lett., B209:214, 1988.

[43] J. F. G. Cascales, M. P. Garcia del Moral, F. Quevedo, and A. M. Uranga. Realistic d-brane models on warped throats: Fluxes, hierarchies and moduli stabilization. JHEP, 02:031, 2004.

[44] Juan F. G. Cascales and Angel M. Uranga. Chiral 4d $\mathrm{n}=1$ string vacua with d-branes and nsns and rr fluxes. JHEP, 05:011, 2003.

[45] S. Chaudhuri, G. Hockney, and J. Lykken. Three generations in the fermionic construction. Nucl. Phys., B469:357-386, 1996.

[46] Mirjam Cvetic, Tianjun Li, and Tao Liu. Supersymmetric pati-salam models from intersecting d6- branes: A road to the standard model. 2004.

[47] Mirjam Cvetic and Ioannis Papadimitriou. More supersymmetric standard-like models from intersecting d6-branes on type iia orientifolds. Phys. Rev., D67:126006, 2003.

[48] Mirjam Cvetic, Ioannis Papadimitriou, and Gary Shiu. Supersymmetric three family su(5) grand unified models from type iia orientifolds with intersecting d6-branes. Nucl. Phys., B659:193-223, 2003.

[49] Mirjam Cvetic, Gary Shiu, and Angel M. Uranga. Chiral four-dimensional $\mathrm{n}=1$ supersymmetric type iia orientifolds from intersecting d6-branes. Nucl. Phys., B615:3-32, 2001.

[50] Mirjam Cvetic, Gary Shiu, and Angel M. Uranga. Three-family supersymmetric standard like models from intersecting brane worlds. Phys. Rev. Lett., 87:201801, 2001.

[51] Atish Dabholkar and Jaemo Park. Strings on orientifolds. Nucl. Phys., B477:701-714, 1996.

[52] Marijn Davidse. Compactifications, boundary states and tadpole equations. Master's thesis, Free university Amsterdam, 2002.

[53] A. de Rújula, H. Georgi, and S. L. Glashow. Trinification of all elementary particle forces. In K. Kang, H. Fried, and P. Frampton, editors, Fifth Workshop on Grand Unification, page 88. World Scientific, Singapore, 1984.

[54] Frederik Denef and Michael R. Douglas. Computational complexity of the landscape. i. 2006.

[55] P. Di Francesco and J. B. Zuber. Su(n) lattice integrable models associated with graphs. Nucl. Phys., B338:602-646, 1990.

[56] Paolo Di Vecchia et al. Classical p-branes from boundary state. Nucl. Phys., B507:259-276, 1997. 
[57] Robbert Dijkgraaf, Cumrun Vafa, Erik P. Verlinde, and Herman L. Verlinde. The operator algebra of orbifold models. Commun. Math. Phys., 123:485, 1989.

[58] Robbert Dijkgraaf, Erik P. Verlinde, and Herman L. Verlinde. C $=1$ conformal field theories on riemann surfaces. Commun. Math. Phys., 115:649-690, 1988.

[59] T. P. T. Dijkstra, L. R. Huiszoon, and A. N. Schellekens. Chiral supersymmetric standard model spectra from orientifolds of gepner models. Phys. Lett., B609:408-417, 2005.

[60] T. P. T. Dijkstra, L. R. Huiszoon, and A. N. Schellekens. Supersymmetric standard model spectra from rcft orientifolds. Nucl. Phys., B710:3-57, 2005.

[61] Ron Donagi, Burt A. Ovrut, Tony Pantev, and Daniel Waldram. Standard models from heterotic m-theory. Adv. Theor. Math. Phys., 5:93-137, 2002.

[62] Michael R. Douglas. The statistics of string / m theory vacua. JHEP, 05:046, 2003.

[63] Alon E. Faraggi. Construction of realistic standard - like models in the free fermionic superstring formulation. Nucl. Phys., B387:239-262, 1992.

[64] Alon E. Faraggi. A new standard - like model in the four-dimensional free fermionic string formulation. Phys. Lett., B278:131-139, 1992.

[65] Alon E. Faraggi, D. V. Nanopoulos, and Ka-jia Yuan. A standard like model in the 4-d free fermionic string formulation. Nucl. Phys., B335:347, 1990.

[66] Giovanni Felder, Jurg Frohlich, Jurgen Fuchs, and Christoph Schweigert. Conformal boundary conditions and three-dimensional topological field theory. Phys. Rev. Lett., 84:1659$1662,2000$.

[67] Giovanni Felder, Jurg Frohlich, Jurgen Fuchs, and Christoph Schweigert. The geometry of wzw branes. J. Geom. Phys., 34:162-190, 2000.

[68] A. Font, Luis E. Ibanez, F. Quevedo, and A. Sierra. The construction of 'realistic' fourdimensional strings through orbifolds. Nucl. Phys., B331:421-474, 1990.

[69] J. Fuchs, L. R. Huiszoon, A. N. Schellekens, C. Schweigert, and J. Walcher. Boundaries, crosscaps and simple currents. Phys. Lett., B495:427-434, 2000.

[70] J. Fuchs, A. N. Schellekens, and C. Schweigert. Galois modular invariants of wzw models. Nucl. Phys., B437:667-694, 1995.

[71] J. Fuchs, A. N. Schellekens, and C. Schweigert. A matrix s for all simple current extensions. Nucl. Phys., B473:323-366, 1996.

[72] Jurgen Fuchs, Albrecht Klemm, Christoph Scheich, and Michael G. Schmidt. Spectra and symmetries of gepner models compared to calabi-yau compactifications. Ann. Phys., 204:151, 1990.

[73] Jurgen Fuchs, Ingo Runkel, and Christoph Schweigert. Tft construction of rcft correlators. iii: Simple currents. Nucl. Phys., B694:277-353, 2004.

[74] Jurgen Fuchs and Christoph Schweigert. A classifying algebra for boundary conditions. Phys. Lett., B414:251-259, 1997.

[75] Jurgen Fuchs and Christoph Schweigert. Branes: From free fields to general backgrounds. Nucl. Phys., B530:99-136, 1998.

[76] Jurgen Fuchs and Christoph Schweigert. Symmetry breaking boundaries. i: General theory. Nucl. Phys., B558:419-483, 1999. 
[77] Jurgen Fuchs and Christoph Schweigert. Symmetry breaking boundaries. ii: More structures, examples. Nucl. Phys., B568:543-593, 2000.

[78] Jurgen Fuchs, Christoph Schweigert, and Johannes Walcher. Projections in string theory and boundary states for gepner models. Nucl. Phys., B588:110-148, 2000.

[79] T. Gannon, P. Ruelle, and M. A. Walton. Spectra of conformal field theories with current algebras. 1995.

[80] Terry Gannon. Towards a classification of $\mathrm{su}(2) \times \ldots \times \mathrm{su}(2)$ modular invariant partition functions. J. Math. Phys., 36:675-706, 1995.

[81] B. Gato-Rivera and A. N. Schellekens. Complete classification of simple current modular invariants for $(\mathrm{z}(\mathrm{p}))^{* *} \mathrm{k}$. Commun. Math. Phys., 145:85-122, 1992.

[82] Doron Gepner. String theory on calabi-yau manifolds: The three generations case. 1987.

[83] Eric G. Gimon and Joseph Polchinski. Consistency conditions for orientifolds and dmanifolds. Phys. Rev., D54:1667-1676, 1996.

[84] Paul H. Ginsparg. Curiosities at c =1. Nucl. Phys., B295:153-170, 1988.

[85] Florian Gmeiner, Ralph Blumenhagen, Gabriele Honecker, Dieter Lust, and Timo Weigand. One in a billion: Mssm-like d-brane statistics. JHEP, 01:004, 2006.

[86] Suresh Govindarajan and Jaydeep Majumder. Crosscaps in gepner models and type iia orientifolds. JHEP, 02:026, 2004.

[87] Brian R. Greene, Kelley H. Kirklin, Paul J. Miron, and Graham G. Ross. A three generation superstring model. 1. compactification and discrete symmetries. Nucl. Phys., B278:667, 1986.

[88] Shahram Hamidi and Cumrun Vafa. Interactions on orbifolds. Nucl. Phys., B279:465, 1987.

[89] Gabriele Honecker. Chiral supersymmetric models on an orientifold of $z(4) \times z(2)$ with intersecting d6-branes. Nucl. Phys., B666:175-196, 2003.

[90] Gabriele Honecker and Tassilo Ott. Getting just the supersymmetric standard model at intersecting branes on the $\mathrm{z}(6)$-orientifold. 2004.

[91] Petr Horava. Strings on world sheet orbifolds. Nucl. Phys., B327:461, 1989.

[92] L. R. Huiszoon, K. Schalm, and A. N. Schellekens. Geometry of wzw orientifolds. Nucl. Phys., B624:219-252, 2002.

[93] L. R. Huiszoon and A. N. Schellekens. Crosscaps, boundaries and t-duality. Nucl. Phys., B584:705-718, 2000.

[94] L. R. Huiszoon, A. N. Schellekens, and N. Sousa. Klein bottles and simple currents. Phys. Lett., B470:95-102, 1999.

[95] L. R. Huiszoon, A. N. Schellekens, and N. Sousa. Open descendants of non-diagonal invariants. Nucl. Phys., B575:401-415, 2000.

[96] L.R. Huiszoon. D-branes and O-planes in string theory: An algebraic approach. PhD thesis, Radboud University Nijmegen, 2002.

[97] Luis E. Ibanez, Jihn E. Kim, Hans Peter Nilles, and F. Quevedo. Orbifold compactifications with three families of $\mathrm{su}(3) \mathrm{x} \mathrm{su}(2) \times \mathrm{u}(1)^{* *}$ n. Phys. Lett., B191:282-286, 1987.

[98] Luis E. Ibanez, F. Marchesano, and R. Rabadan. Getting just the standard model at intersecting branes. JHEP, 11:002, 2001. 
[99] Nobuyuki Ishibashi. The boundary and crosscap states in conformal field theories. Mod. Phys. Lett., A4:251, 1989.

[100] Katsumi Itoh, Mitsuhiro Kato, Hiroshi Kunitomo, and Makoto Sakamoto. Vertex construction and zero modes of twisted strings on orbifolds. Nucl. Phys., B306:362, 1988.

[101] Shamit Kachru. Some three generation (0,2) calabi-yau models. Phys. Lett., B349:76-82, 1995.

[102] Zurab Kakushadze, Gary Shiu, S. H. Henry Tye, and Yan Vtorov-Karevsky. A review of three-family grand unified string models. Int. J. Mod. Phys., A13:2551-2598, 1998.

[103] Yoichi Kazama and Hisao Suzuki. New n=2 superconformal field theories and superstring compactification. Nucl. Phys., B321:232, 1989.

[104] Elias B. Kiritsis. Proof of the completeness of the classification of rational conformal theories with $\mathrm{c}=1$. Phys. Lett., B217:427, 1989.

[105] Christos Kokorelis. Standard model compactifications from intersecting branes. 2002.

[106] Christos Kokorelis. N = 1 locally supersymmetric standard models from intersecting branes. 2003.

[107] Boris Kors and Pran Nath. A stueckelberg extension of the standard model. Phys. Lett., B586:366-372, 2004.

[108] Boris Kors and Pran Nath. A supersymmetric stueckelberg u(1) extension of the mssm. JHEP, 12:005, 2004.

[109] Boris Kors and Pran Nath. Aspects of the stueckelberg extension. JHEP, 07:069, 2005.

[110] M. Kreuzer and A. N. Schellekens. Simple currents versus orbifolds with discrete torsion: A complete classification. Nucl. Phys., B411:97-121, 1994.

[111] Maximilian Kreuzer and Harald Skarke. Complete classification of reflexive polyhedra in four dimensions. Adv. Theor. Math. Phys., 4:1209-1230, 2002.

[112] G. K. Leontaris and J. RIzos. A d-brane inspired trinification model. 2006.

[113] W. Lerche, A. N. Schellekens, and N. P. Warner. Lattices and strings. Phys. Rept., 177:1, 1989.

[114] C. A. Lutken and G. G. Ross. Symmetries and couplings in heterotic superconformal field theories. Phys. Lett., B214:357, 1988.

[115] J. F. Mathiot, V. A. Karmanov, and A. V. Smirnov. Non-perturbative renormalization in light front dynamics with fock space truncation. 2005.

[116] Joseph Polchinski. Evaluation of the one loop string path integral. Commun. Math. Phys., 104:37, 1986.

[117] Joseph Polchinski. Dirichlet-branes and ramond-ramond charges. Phys. Rev. Lett., 75:47244727, 1995.

[118] G. Pradisi, A. Sagnotti, and Ya S. Stanev. Planar duality in su(2) wzw models. Phys. Lett., B354:279-286, 1995.

[119] G. Pradisi, A. Sagnotti, and Ya. S. Stanev. Completeness conditions for boundary operators in 2d conformal field theory. Phys. Lett., B381:97-104, 1996.

[120] Gianfranco Pradisi and Augusto Sagnotti. Open string orbifolds. Phys. Lett., B216:59, 1989. 
[121] A. Recknagel and V. Schomerus. D-branes in gepner models. Nucl. Phys., B531:185-225, 1998.

[122] Andreas Recknagel and Volker Schomerus. Boundary deformation theory and moduli spaces of d-branes. Nucl. Phys., B545:233-282, 1999.

[123] I. Runkel and G. M. T. Watts. A non-rational cft with central charge 1. Fortsch. Phys., 50:959-965, 2002.

[124] Augusto Sagnotti. Open strings and their symmetry groups. In G. 't Hooft, A. Jaffe, G. Mack, P.K. Mitter, and Stora. R., editors, Non-Perturbative Quantum Field Theory, 1987.

[125] Augusto Sagnotti and Yassen S. Stanev. Open descendants in conformal field theory. Fortsch. Phys., 44:585-596, 1996.

[126] A. N. Schellekens. unpublished, 1990.

[127] A. N. Schellekens. Electric charge of quantization in string theory. Phys. Lett., B237:363, 1990.

[128] A. N. Schellekens. Cloning so(n) level 2. Int. J. Mod. Phys., A14:1283-1292, 1999.

[129] A. N. Schellekens. Fixed point resolution in extended wzw-models. Nucl. Phys., B558:484502, 1999.

[130] A. N. Schellekens. The landscape 'avant la lettre'. 2006.

[131] A. N. Schellekens and N. Sousa. Open descendants of u(2n) orbifolds at rational radii. Int. J. Mod. Phys., A16:3659-3672, 2001.

[132] A. N. Schellekens and Ya. S. Stanev. Trace formulas for annuli. JHEP, 12:012, 2001.

[133] A. N. Schellekens and S. Yankielowicz. Exceptional modular invariants of $\mathrm{n}=2$ tensor products. Phys. Lett., B242:45, 1990.

[134] A. N. Schellekens and S. Yankielowicz. New modular invariants for $n=2$ tensor products and four-dimensional strings. Nucl. Phys., B330:103, 1990.

[135] N. Sousa and A. N. Schellekens. Orientation matters for nimreps. Nucl. Phys., B653:339$368,2003$.

[136] Leonard Susskind. The anthropic landscape of string theory. 2003.

[137] Xiao-Gang Wen and Edward Witten. Electric and magnetic charges in superstring models. Nucl. Phys., B261:651, 1985.

[138] Edward Witten. Strong coupling expansion of calabi-yau compactification. Nucl. Phys., B471:135-158, 1996.

[139] Edward Witten. Toroidal compactification without vector structure. JHEP, 02:006, 1998. 


\section{Summary}

Since the dawn of time, mankind has wanted to understand the world around him. In the last centuries scientists have been making great progress in this endeavour by studying different aspects of our world. Understanding the ever smaller building blocks of matter, forces, space and time has been the focus of theoretical high energy physicists. Together with experimental input, this research has lead to accurate descriptions of these building blocks.

For example, for matter we now understand that there is whole hierarchy of scales; from grains of material, to molecules, atoms, nuclear particles (neutrons and protons), quarks and electrons. And there is where it ends - for now. For this last scale, that of the quarks and electrons, we have a theory that describes these building blocks and their interaction with those of (all but one of) the forces very well. It describes which particles exist in nature and how they are charged. A charge specifies how a particle 'feels' a force. Particles can for example be positively or negatively charged with respect to the electrical force. For the other forces more generalized notions of charge exist. The theory that describes all this is known as the Standard Model of particle physics. But this theory doesn't describe all building blocks. Since Einstein created his theory of General Relativity, we know that space and time are intimately related to gravity; the force massive objects exert on one another can be described by the curvature of space and time.

Our description of matter in combination with gravity ceases to work at even smaller distance scales than that of the Standard Model. To overcome these problems physicists are looking for a theory of Quantum Gravity, which should combine both the theory of gravity and the Standard Model in one theory. To date, the only serious candidate is String Theory. One of the reasons physicists started to consider String Theory as a theory of both the Standard Model particles and Gravity is that early on it was clear that it contains the quantum aspects of gravity. Recovering the correct number and kinds of matter and force particles has, however, remained an open problem for many years.

We now think that there is only one String Theory. Although there exist various seemingly different formulations, they are all just descriptions of the same thing. In formulations of String Theory that were most popular right after its conception (in which strings are always closed), various constructions were found that resembled the Standard Model. They all had their problems however, and once the full potential of open strings were discovered, interest shifted to constructing open string theories that contained the Standard Model particles. Until the work in this thesis this project was not successful. 
In String Theory all elementary particles, both the matter and the force particles, are described by vibrating strings. In String Theory there are no further, more fundamental building blocks. String is the 'stuff' everything is made of. The different kinds of particles must then be differentiated by something else. One such property is the mode of vibration.

In most string theories however, everything but the lowest tone has a mass much higher than any of the particles we know. The lowest tones are actually massless, precisely like the matter particles in the Standard Model which are essentially massless, but get their mass from interaction with the Higgs particle. Fortunately there are other properties which can be used to distinguish different particles. Strings propagate through some background space, which in general can have various dimensions and even loops. Such a space can contain surfaces $^{13}$ (which are called Branes in String Theory) in different locations on which open strings can end. The mass of a string stretched between two branes is dependent on the distance between two branes. Only when the length of the string is very small, where the two branes intersect, the string can be massless. It turns out that each of these branes corresponds to a force and the label that identifies to which brane a string is attached, corresponds to the charges of the string with respect to that force. A string wrapped around a certain loop in space or attached to some surface is different from one that does not. This means that the shape of the space in which the strings can move and the branes that exist in these spaces (collectively named the background) determine the properties of the particles that can exist. If a certain space has the types of branes that correspond to the forces of the Standard Model, than a string stretched between, say, surface $a$ and $b$ can be a quark and between $b$ and $c$ an electron.

The properties of the background through which strings can propagate are not arbitrary. String Theory calculations simply do not make sense for spaces that do not meet certain criteria. First of all the space has to be ten dimensional, of which of course a four dimensional subsurface must look like the four dimensions we observe. Furthermore one can not just choose the number and positions of the branes. In practice there are additional restrictions coming from the fact that only for specific spaces are calculations computationally possible. So although in principle we know what configurations of branes we need to get the correct particles, this does not mean we have found a correct String Theory description yet. The technical description of strings propagating on such a space is given by what is called a Conformal Field Theory (CFT).

Several methods have been devised to find and construct different configurations of branes. A method that has gotten and still gets much attention is the so called geometric method. In this approach one chooses a suitable ten dimensional (usually fairly simple) space, then finds out what possible branes it admits and tries to find a construction that has the right particles in it.

The algebraic method, used in this thesis, is different. One can forget the interpretation of strings propagating on a space and just focus on finding a suitable CFT. Although the

${ }^{13}$ And their higher dimensional analogues 
interpretation in terms of branes in a certain space is a bit fuzzy, one can still calculate which particles are present. There are still labels that distinguish the different particle species.

The great advantage of this method is that one can use CFTs which correspond to highly non-trivial spaces, inaccessible to the previous method, but nonetheless relatively easy to work with. Finding such CFTs is not a trivial job however. Just like there are restrictions on the branes one can put in a space, there are restrictions on the boundary and cross-cap states in a CFT. Fortunately there are methods that allow one to construct theories with open unoriented (meaning that the strings do not have a direction) strings from theories that have only closed oriented ones. The latter ones are more easily constructed. In chapter 2 we review what restrictions exist for theories with only closed strings and how a theory containing open unoriented strings can be derived from such a parent theory.

In some cases both algebraic and geometric methods can be applied to certain spaces. In chapter 3 we analyse one particular example of a parent closed string theory. We find all possible unoriented open string theories (orientifolds) one can construct from the closed string theory in both schemes. We also try to use the geometric approach to get more intuition for the boundary states obtained in the algebraic approach.

By using the algebraic method we have access to a large set of backgrounds, each of which leads to different types of particle. Choosing a space fixes the possible branes and their intersections. We can however still decide if they appear at all, and to some degree also what charges they correspond to. This opens up the possibility to search for backgrounds containing the types of particle we see in nature. In chapter 5 we report on the first such search conducted, and the actual first (and the next 211,633) open string theories that have the correct types of particle.

It is a actually a subtle issue which particles should be allowed in the models described above because we simply do not know the complete list of all particles that exist. Of course the Standard Model particles are present, but even then one of the particles in the Standard Model, the Higgs particle, has not been observed yet. There is also strong evidence for particles that are not described by the Standard Model, the so called dark matter. This is matter that is only detected by its gravitational interaction. It is roughly 5 times as abundant as the matter we are made of, the Standard Model particles. Some hypothetical types of particles can be excluded because we should have seen them in our detectors by now. These particles clearly should not exist in our models. But for very heavy particles it is impossible to prove they do not exist. In our search we left the number of these unknown particles free and studied how many occur in the models we found.

Finally there are possibilities to generalize the configurations we searched for so far. For instance, the forces we know could be part of a unified force. One peculiar feature of the spectrum of matter particles we see in nature is that they all come in three copies which only differ by their mass. For instance there are three types of 'electrons'. Everything around us is made up from the lightest kind, but in accelerators and astrophysical systems also the heavier types are created. In the first search this multiplicity came from multiple intersections between two branes. Another possibility is to introduce different branes for different copies. All these possibilities were included in a second search where no pattern 
of intersections was a priori assumed. A lot of different models were found, with varying numbers of occurrences. In the last chapter we present this search and analyse why certain models occur more often than others or even not at all. We find that the pattern of intersection between the various branes is an important factor. In general patterns with more intersections are harder to find.

Finding the first open String Theory backgrounds that reproduce the Standard Model particles is a nice first step. There is however still much to do. The next step in understanding how the Standard Model fits in String Theory will probably come from a statistical approach in the spirit of the analysis of intersection numbers we have done in the last chapter. 


\section{Samenvatting}

$\mathrm{Al}$ zo lang de mensheid bestaat heeft ze de wereld om zich heen proberen te begrijpen en beschrijven. In de afgelopen eeuwen hebben wetenschappers grote vooruitgang geboekt in deze queeste door veel verschillende aspecten van onze wereld te onderzoeken. Het begrijpen van de steeds kleinere bouwstenen van materie, krachten, ruimte en tijd is een van de doelen van de theoretische natuurkunde. Samen met experimentele input heeft theoretisch onderzoek geleid tot een zeer nauwkeurige beschrijving van deze bouwstenen.

Voor materie - bijvoorbeeld - begrijpen we nu dat er een hiërarchie van steeds kleinere eenheden is, van korrels tot moleculen, atomen, kerndeeltjes (neutronen en protonen), quarks en elektronen. En daar stopt het - voor nu. Voor de laatste schaal, die van de elektronen en de quarks, hebben we een theorie die deze bouwstenen en de interacties met die van (alle behalve een van) de krachten zeer goed beschrijft. Deze theorie beschrijft welke deeltjes er bestaan in de natuur en hoe die geladen zijn. De lading van een deeltje beschrijft hoe het een kracht 'voelt'. Deeltjes kunnen bijvoorbeeld positief of negatief geladen zijn met betrekking tot de elektrische kracht. Voor de andere krachten bestaat een gegeneraliseerde notie van lading. De theorie die dit alles beschrijft, staat bekend als het standaardmodel van de deeltjesfysica. Deze theorie beschrijft echter niet alle bouwstenen. Sinds Einstein zijn algemene relativiteitstheorie opschreef, weten we dat ruimte en tijd nauw verbonden zijn met zwaartekracht. De kracht die massieve objecten op elkaar uitoefenen kan beschreven worden door de kromming van ruimte-tijd.

Onze beschrijving van materie in combinatie met zwaartekracht verliest zijn geldigheid bij afstandsschalen die vele malen kleiner zijn dan die waarvoor het standaardmodel relevant is. Om deze problemen de baas te worden zijn natuurkundigen op zoek naar een theorie van quantumzwaartekracht. Deze theorie moet het standaardmodel en Einsteins zwaartekracht theorie combineren tot één nieuwe theorie. Tot op heden is de enige serieuze kandidaat, de snaartheorie. Een van de redenen waarom natuurkundigen snaartheorie zien als een serieuze kandidaat als theorie voor zowel de standaardmodeldeeltjes als voor de zwaartekracht, is dat het vrij snel duidelijk was dat het de quantumaspecten van zwaartekracht bevat. Het terugvinden van het correcte aantal en het goede type voor de materie- en krachtdeeltjes bleef een open vraag gedurende vele jaren.

We denken nu dat er maar één snaartheorie is. Ondanks het feit dat er verscheidene - op het eerst gezicht verschillende - formuleringen zijn, zijn ze slechts beschrijvingen van hetzelfde ding. In de formuleringen van snaartheorie die vlak na haar geboorte in zwang waren (waarin snaren altijd gesloten zijn), zijn verschillende constructies gevonden die 
lijken op het standaardmodel. Ze hadden echter allemaal een of meerdere serieuze problemen, en toen het volledig potentieel van open snaren duidelijk werd, verschoof de aandacht naar het construeren van het standaardmodel met behulp van opensnaartheorieën. Een project dat tot het werk uit dit proefschrift niet succesvol was.

In snaartheorie worden alle elementaire deeltjes - zowel materie- als krachtdeeltjes beschreven door trillende snaren. In snaartheorie bestaat geen, fundamenteler bouwsteen. Snaar is het 'spul' waar alles van gemaakt is. De verschillende typen deeltjes moeten daarom onderscheiden worden door iets anders. Een zo'n eigenschap is de trillingsmodus.

In de meeste snaartheorieën is alles - behalve de laagste 'toon' - veel zwaarder dan het zwaarste deeltje dat we kennen. De deeltjes die corresponderen met de laagste toon zijn massaloos. Dit is net zoals de deeltjes van het standaardmodel, die op het meest fundamentele niveau massaloos zijn en hun massa krijgen van interactie met het Higgsdeeltje. Gelukkig zijn er andere eigenschappen die we kunnen gebruiken om deeltjes te onderscheiden. Snaren bewegen zich voort door een achtergrond-ruimte, die een groot aantal dimensies kan hebben en zelfs 'handvatten'. Deze ruimtes kunnen oppervlakken ${ }^{14}$ (die branen genoemd worden in snaartheorie) bevatten op verschillende plekken en momenten waaraan snaren vast kunnen zitten. De massa van een snaar gespannen tussen twee branen is afhankelijk van de afstand tussen de branen. Alleen als de lengte van de snaar heel klein wordt, waar de branen snijden, kan de snaar massaloos zijn. Het blijkt dat elke braan correspondeert met een kracht. Een snaar die eindigt op zo'n braan is geladen met betrekking tot die kracht. Een snaar die vast zit aan een braan of gewonden is om een handvat in de ruimte is anders dan een snaar die dat niet is. Dit betekent dat de eigenschappen van de ruimte waarin de string beweegt en de branen die zich erin bevinden (tezamen de achtergrond) de eigenschappen bepalen van de deeltjes die zich in de ruimte kunnen bevinden. Als een bepaalde achtergrond bijvoorbeeld de typen branen heeft die overeenkomen met de krachten in het standaardmodel, dan is een snaar die zich strekt tussen - laten we aannemen - oppervlak $a$ en $b$ een quark en tussen $b$ en $c$ een elektron.

De eigenschappen van de achtergrond zijn niet arbitrair. Berekeningen in snaartheorie slaan nergens op als ruimtes niet voldoen aan bepaalde criteria. In de eerste plaats moet de ruimte tiendimensionaal zijn, waarvan natuurlijk een vierdimensionale subruimte eruit moet zien als de vier dimensies die we om ons heen waarnemen. Vervolgens kan men niet zomaar het aantal en de posities van de branen kiezen. In de praktijk komen er nog extra restricties bij: alleen voor bepaalde typen ruimtes zijn de berekeningen ook computationeel uitvoerbaar. Dus ondanks het feit dat we weten wat voor een configuratie van branen we nodig hebben om de goede deeltjes te krijgen, betekent dit niet dat we al een snaartheoriebeschrijving van het standaardmodel hebben. De technische beschrijving van snaren die bewegen in zulke ruimtes wordt gegeven door een zogenaamde conforme velden theorie (CFT).

Er zijn verschillende methoden bedacht om configuraties van branen te vinden en construeren. Een methode die veel aandacht heeft gekregen en dat nog steeds krijgt, is de

\footnotetext{
${ }^{14}$ En het hogerdimensionaal equivalent daarvan.
} 
zogenoemde geometrische methode. In deze aanpak kiest men een geschikte tiendimensionale ruimte (meestal een vrij eenvoudige), dan zoekt men uit welke branen zijn toegestaan en op welke posities. Uiteindelijk probeert men een constructie te maken met de juiste typen en aantallen deeltjes.

De algebraïsche methode die gebruikt wordt in dit proefschrift, is anders. Het is mogelijk om de interpretatie van snaren die bewegen door de ruimte te verlaten en zich volledig te concentreren op het vinden van een bruikbare CFT. Ondanks het feit dat de interpretatie in termen van branen in een ruimte vaag is, is het mogelijk om uit te rekenen welke type deeltjes er zijn. Er zijn nog steeds labels die het mogelijk maken om verschillende soorten deeltjes te onderscheiden.

Het grote voordeel van deze methode is dat het mogelijk is om CFT's te gebruiken die overeenkomen met zeer ingewikkelde ruimten, die ontoegankelijk zijn voor de voorgaande methode, terwijl deze CFT's relatief makkelijk zijn om mee te werken. Het vinden van zo'n ruimte is echter niet eenvoudig. Net zoals er beperkingen zijn op de branen die men in een bepaalde ruimte kan plaatsen, zijn er beperkingen op de boundary-en cross-captoestanden in een CFT. Gelukkig zijn er methoden om een achtergrond met open ongeoriënteerde snaren (snaren zonder richting) te construeren van een achtergrond die alleen gesloten snaren heeft. Dit laatste type achtergrond is veel eenvoudiger te construeren. In hoofdstuk 2 bespreek ik welke beperkingen er bestaan voor theorieën met enkel gesloten snaren en hoe een theorie die open ongeoriënteerde snaren bevat, kan worden afgeleid van een dergelijke oudertheorie.

In sommige gevallen kan zowel de algebraïsche als de geometrische methode worden toegepast op een ruimte. In hoofdstuk 3 analyseren we een voorbeelda van een dergelijke oudertheorie. We vinden met beide methoden al de mogelijke achtergronden met ongeoriënteerde open snaren (orientifolds) die kunnen worden afgeleid van die met enkel gesloten snaren. We proberen ook met behulp van de geometrische methode meer intuïtie te krijgen voor de boundary-toestanden verkregen in de algebraïsche methode.

Door gebruik te maken van de algebraïsche methode hebben we toegang tot een grote set van achtergronden, die in het algemeen elk weer tot andere deeltjes leiden. Het kiezen van een bepaalde ruimte legt de toegestane branen en hun doorsneden vast. We kunnen echter nog wel kiezen óf de branen voorkomen en tot op bepaalde hoogte met welke ladingen ze corresponderen. Dit geeft ons de mogelijkheid om specifiek naar achtergronden te zoeken die de typen deeltjes bevatten die we in de natuur zien. In hoofdstuk 5 doen we verslag van de eerste zoektocht van dit type dat is uitgevoerd, en van de eerste opensnaarachtergrond (en de volgende 211.633) die ermee gevonden is.

Welke deeltjes men toestaat in de zojuist besproken modellen is minder triviaal dan het lijkt. Uiteindelijk komt dit omdat we niet weten of we de complete lijst van deeltjes die voorkomen in de natuur kennen. Natuurlijk moeten de standaardmodeldeeltjes aanwezig zijn in elk realistisch model. Maar zelfs van het standaardmodel is er nog één deeltje, het Higgs-deeltje, dat nog nooit direct is geobserveerd. Er zijn ook sterke aanwijzingen voor deeltjes die niet in het standaardmodel voorkomen, de zogenaamde donkere materie. Dit is materie die alleen wordt waargenomen door de zwaartekracht die zij uitoefent. Van deze donkere materie is er ongeveer vijf keer zoveel als van het spul waar wij van gemaakt zijn, 
namelijk de standaardmodeldeeltjes. Sommige soorten hypothetische deeltjes kunnen worden uitgesloten, omdat ze anders wel in onze detectoren zouden moeten zijn waargenomen. Deze deeltjes mogen natuurlijk bestaan in onze modellen. Voor andere, zeer zware deeltjes is het onmogelijk om ze uit te sluiten. In onze zoektocht hebben we de aantallen van deze typen deeltjes vrijgelaten en bestudeerd hoe vaak ze voorkomen in de gevonden modellen.

Tot slot zijn er nog mogelijkheden om de constructies waarnaar we zoeken te generaliseren. Zo is het bijvoorbeeld mogelijk dat de krachten die we kennen, een onderdeel zijn van een grotere, geünificeerde kracht. Een andere komt van de mogelijke verklaring van een opvallend patroon in de typen deeltjes die voorkomen in de natuur. De materiedeeltjes komen namelijk allemaal voor in drievoud, die onderling alleen verschillen in hun massa. Er zijn zo bijvoorbeeld drie typen 'elektronen'. Alles wat we om ons heen zien is opgebouwd uit de lichtste soort, maar in deeltjesversnellers en astrofysische systemen worden de zwaardere typen ook gecreëerd. In de eerste zoektocht is het drievoud het gevolg van meerdere doorsneden tussen dezelfde branen. Een andere mogelijkheid is het introduceren van een braan voor elke kopie.

$\mathrm{Al}$ deze mogelijkheden zijn meegenomen in een tweede zoektocht waarin geen patroon van doorsneden a priori is aangenomen. We hebben veel verschillende modellen - in sterk variërende aantallen - gevonden. In het laatste hoofdstuk presenteren we deze zoektocht en analyseren we waarom bepaalde modellen vaker voorkomen dan andere, of juist helemaal niet. We vinden dat het patroon van doorsneden tussen de verschillende branen een belangrijke factor is. Zo zijn bijvoorbeeld in het algemeen patronen met meer doorsneden moeilijker te vinden.

Het vinden van de eerste opensnaarachtergronden die de deeltjes van het standaardmodel reproduceren is een aardige eerste stap. Er is echter nog veel te doen. De volgende stap in het begrijpen hoe het standaardmodel past in snaartheorie zal waarschijnlijk komen van een statistische aanpak in de geest van de analyse van de intersectie getallen zoals uitgevoerd in het laatste hoofdstuk. 


\section{Dankwoord}

Aan de totstandkoming van dit proefschrift hebben - direct of indirect - veel mensen bijgedragen. Daar wil ik ze graag voor bedanken. In de eerste plaats natuurlijk mijn promotor, Bert Schellekens. Ik had geluk dat ik op het juiste moment bij zijn onderzoek inhaakte. Door geduldige uitleg en verhelderende discussies kreeg ik de mogelijkheid om me in te werken in de materie en samen de eerste standaardmodellen te vinden. Zonder zijn ideeën, advies en hulp was dit proefschrift er nooit gekomen. Verder ben ik natuurlijk veel dank verschuldigd aan de andere mensen met wie ik samen onderzoek gedaan heb. In het bijzonder wil ik Fabio Riccioni en Lennaert Huiszoon bedanken voor interessante discussies en nuttig commentaar op teksten die ik heb geschreven. De laatste ben ik nog eens extra dankbaar voor het kritisch lezen van het proefschrift. Ik wil Eva Smal, Hylke Koers en Chris White bedanken voor het commentaar op de Engelse samenvatting, Noor en John Smal voor het commentaar op de Nederlandse vertaling daarvan en Charlotte Dijkstra en Dieuwertje Smal voor de omslag.

Erg belangrijk is ook de dagelijkse sfeer op het Nikhef geweest. Gelukkig had ik fijne kamergenoten en collega's. Met Andrea Fuster heb ik altijd lekker kunnen kletsen, natuurlijk veel over Tristi en Sybri. Hylke wil ik bedanken voor de leuke discussies die we gehad hebben over allerhande (natuurkundige) onderwerpen. Erg leuk vond ik het ook om samen te werken aan ons kink-projectje, helaas hebben we het niet kunnen uitwerken tot een publicatie. Misschien niet direct nuttig voor het afmaken van het proefschrift, maar wel voor het ordenen van de gedachten waren de gesprekken met Alejandro Arrizabalaga over onze twijfels over toekomstige banen en andere moeilijke beslissingen. Verder wil ik natuurlijk alle andere collega's van de theoriegroep bedanken voor de gezelligheid en de discussies bij de thee/lunch.

Maar op het moment dat je naar huis gaat wacht nog een hele andere wereld die minstens zo belangrijk is om de inspiratie te vinden om je werk te kunnen doen. Zo zijn er natuurlijk mijn vrienden Edo Koopman, Job Raaijmakers, Joris Leker en Tjeerd Boonstra met wie ik tijdens onze jaarlijkse zeiltochten overdag een frisse wind door m'n hoofd kon laten waaien om die 's nachts weer te benevelen. En Marijn van der Velde, die er op beslissende momenten was om een goed gesprek mee te voeren. Veel steun heb ik ook gehad van mijn familie in de brede zin, mijn schoonfamilie en natuurlijk mijn zus en broers. Erg stimulerend waren bijvoorbeeld de opmerkingen van mijn kleine broertjes, toen die aan hun eerste vaste baan begonnen, over wat ik allemaal wel niet deed met hun belastingcenten!

En dan mijn lieve ouders, die altijd vertrouwen in me hadden en me gestimuleerd hebben: Anneke, dat ik zoveel mogelijk uit mezelf moest halen, en Ton, dat het ook wel een keer af moest.

Als laatste en als belangrijkste, wil ik Willemijn en Sybrand bedanken. Ze zijn mijn heerlijk thuis waar ik kon rusten - of juist nog even met duplo moest spelen - als ik moe was, waar ik afleiding vond na een dag (of een week) vastzitten of de ondersteuning vond die ik nodig had om door te gaan. 


\section{Curriculum Vitae}

Tim Pieter Tjipko Dijkstra, met zijn tweede en derde naam genoemd naar zijn grootvaders, werd geboren in Hattem op vrijdag 13 januari 1978. Hij groeide gelukkig op in een gezin met drie andere kinderen: een grote zus en twee jongere tweelingbroers.

Toen Tim 2 jaar oud was verhuisde het gezin Dijkstra naar Rhenen, een meer centraal gelegen plaats in verband met het werk van vader Dijkstra. Tim ging naar de Katholieke basisschool 'Cunera' in zijn woonplaats. Op zijn eerste schooldag ontmoette hij daar zijn vriend Tjeerd Boonstra. Bij het verlaten van de Cuneraschool, verliet Tim ook de kerk en vervolgde zijn leven als atheïstisch nihilist.

Hij vervolgde zijn opleiding op de middelbare school Het Wagenings Lyceum, waarvoor hij elke ochtend met zijn vriend Joris Leker vanuit Rhenen naar Wageningen fietste. 'het Wagenings', dat toen nog bekend stond om zijn vooruitstrevende staf en bijzondere programma, was voor Tim een inspirerende plek. Tijdens zijn schooltijd was Tim ook voorzitter van de scholierenvereniging het Soet Versaemen. Vele sterke verhalen uit deze tijd komen ook voort uit de uitwisseling met Servische, Kroatische en Bosnische jongeren afkomstig uit een verwoest Joegoslavië en buitenlandse reizen naar Denemarken, Tsjechië, Slowakije en Istanbul.

Op school ontstond de vriendenclub van 5, Tjeerd Boonstra, Joris Leker, Job Raaijmakes, Edo Koopman en natuurlijk Tim. De vriendschap bestaat nog steeds, en wordt elk jaar gevierd met een zeilvakantie die steeds gevaarlijker vormen begint aan te nemen (benieuwd hoe dat zal gaan met al die nieuwe huisvaders).

Tims eerste studiekeuze was Sterrenkunde aan de Universiteit van Amsterdam. Ver weg, dus tijd om het ouderlijk huis te verlaten en in de grote stad te gaan wonen. De woningnood was als altijd hoog, en Tim vond een plekje in de Bijlmer. In het eerstejaar sterrenkunde komt Tim erachter dat zijn echte interesse ligt in de grotere vragen die spelen in de natuurkunde. Grote verbindende theorieën die tegelijkertijd het grootste en het kleinste, het onbereikbare verleden en het heden kunnen verklaren (maar niet vanzelf alles wat ertussen ligt). Daarom stapt hij over naar de natuurkunde. Hij besluit af te studeren bij Karel-Jan Schoutens op een scriptie met de titel Field theoretic description of quantum Hall systems. Daarnaast houdt Tim zich bezig met de kwaliteit en de ontwikkeling van het onderwijs door lid te zijn van de onderwijs- en curriculumcommissie van de piepjonge faculteit Wiskunde, Informatica, Natuurkunde en Sterrenkunde en later de studentenraad tijdens de oprichting van de logge bètafaculteit.

Op de meest waarschijnlijke leeftijd ontmoet hij zijn latere vrouw, Willemijn Smal, rmet wie hij na enkele jaren verkering gaat samenwonen, in de Indische buurt. Later krijgt het stel de kans op stand te gaan wonen op het KNSM-eiland omdat het oude huis op de slooplijst staat.

Nog steeds bewogen door de eerdergenoemde grote vragen maakt Tim de overstap naar de Stringtheorie bij de keuze van zijn promotieonderzoek, en hij krijgt een positie op dit gebied bij Bert Schellekens op het Nikhef. Een drietal jaren geeft Tim het werkcollege Quantum II.

Nog tijdens zijn promotie en in de bloei van zijn vruchtbare leven doet Tim zijn best 
met Willemijn voor nageslacht te zorgen. Sybrand Tame komt ter wereld in juli 2004.

Op dit moment heeft Tim de wetenschap verlaten en heeft hij gekozen voor een zekerder bestaan dan de wetenschap hem kon bieden. De liefde voor het leven gaat niet altijd samen met de jacht op de moderne heilige graal.

Willemijn Smal 